\title{
Towards A Better Understanding Of Paleoindian Native American Settlement In Southern Ohio: A Multi-Scalar Approach
}

Matthew P Purtill

Follow this and additional works at: https://researchrepository.wvu.edu/etd

\section{Recommended Citation}

Purtill, Matthew P, "Towards A Better Understanding Of Paleoindian Native American Settlement In Southern Ohio: A Multi-Scalar Approach" (2018). Graduate Theses, Dissertations, and Problem Reports. 7232.

https://researchrepository.wvu.edu/etd/7232

This Dissertation is protected by copyright and/or related rights. It has been brought to you by the The Research Repository @ WVU with permission from the rights-holder(s). You are free to use this Dissertation in any way that is permitted by the copyright and related rights legislation that applies to your use. For other uses you must obtain permission from the rights-holder(s) directly, unless additional rights are indicated by a Creative Commons license in the record and/ or on the work itself. This Dissertation has been accepted for inclusion in WVU Graduate Theses, Dissertations, and Problem Reports collection by an authorized administrator of The Research Repository @ WVU.

For more information, please contact researchrepository@mail.wvu.edu. 


\title{
TOWARDS A BETTER UNDERSTANDING OF PALEOINDIAN NATIVE AMERICAN SETTLEMENT IN SOUTHERN OHIO: A MULTI-SCALAR APPROACH
}

\author{
Matthew P. Purtill \\ Dissertation submitted \\ to the Eberly College of Arts \& Sciences \\ at West Virginia University \\ in partial fulfillment of the requirements for the degree of \\ Doctor of Philosophy in \\ Geology \& Geography \\ J. Steven Kite ${ }^{1}$, Ph.D., Chair \\ Trevor Harris ${ }^{1}$, Ph.D \\ Jamison Conley ${ }^{1}$, Ph.D. \\ James Thompson ${ }^{2}$, Ph.D. \\ Mark Seeman ${ }^{3}$, Ph.D. \\ ${ }^{1}$ Department of Geology \& Geography, West Virginia University \\ ${ }^{2}$ Division of Plant \& Soil Sciences, West Virginia University \\ ${ }^{3}$ Department of Anthropology, Kent State University \\ Morgantown, West Virginia \\ 2018
}

Keywords: GIS and statistical modeling, Paleoindian archaeology, geomorphology, aeolian sedimentology, Sandy Springs archaeological site, upper Ohio River Valley

Copyright 2018, Matthew P. Purtill 


\title{
ABSTRACT \\ TOWARDS A BETTER UNDERSTANDING OF PALEOINDIAN NATIVE AMERICAN SETTLEMENT IN SOUTHERN OHIO: A MULTI-SCALAR APPROACH
}

\author{
Matthew P. Purtill
}

Despite Ohio's prominent role in the historical trajectory of archaeological research into the Native American Paleoindian period (13.5 to 11.4 ka years ago), it has been over 30 years since the last attempt to model Paleoindian land-use behavior at a resolution that includes the southern half of the state. This dissertation revisited Paleoindian adaptation and land-use behavior in southern Ohio and by association the eastern United States. Research was accomplished through a study of Paleoindian occupation at two distinct resolutions in southern Ohio: (i) regional; and, (ii) local, or site-level. The regional level was explored through a GIS and statistical modeling approach of known Paleoindian site locations against randomly selected environmental information to evaluate current theories of Paleoindian land-use behavior. Significant patterns were further explored through comparison of similarly generated trends for later archaeological sites dated to the Early Archaic ( 11-8.5 ka) and Late Archaic ( $\sim 6-2.7 \mathrm{ka})$ periods. A binary logistical regression test was calculated with significant variables to assess the relative strength of individual variables in explaining Paleoindian site location.

The second, site-level component of this dissertation involved archaeological, geomorphological, and geochronological investigation of the Sandy Springs archaeological site and surrounding landscape. Research focused on landform reconstruction with special emphasis on aeolian sand dune formation histories. Furthermore, proxy paleoenvironmental indicators are documented through investigation of sediments at Sandy Springs. Research focused on potential explanations for site function during the Paleoindian period and directly tested the statement that saline springs were a primary reason for Paleoindian occupation.

Study results indicate that Paleoindians did not practice a uniform land-use strategy in the upper Ohio Valley but instead adapted strategies to fit local conditions. A potentially distinct Paleoindian land-use strategy is identified for the Interior Low Plateaus province of Ohio. This strategy may reflect strengthening post-11 ka interaction for groups occupying the Cumberland, Tennessee, and upper Ohio drainages. This strategy also may indicate a preference for prairie environments. The combined modeling of Paleoindian, Early Archaic, and Late Archaic site distributions provides a method to assess the impact of collector bias on archaeological interpretations. Study results suggest that collector bias is not restricted to Paleoindian assemblages alone. Cross-temporal approaches provide a means to mitigate the influence of such bias in future studies.

Sandy Springs represents one of the largest Paleoindian sites in the upper Ohio River Valley and study results increased our understanding of its geomorphological and archaeological history. The previous claim of saline springs at Sandy Springs was tested through electrical conductivity 
and $\mathrm{pH}$ analysis. Results failed to support the idea that Sandy Springs possessed water salinity at levels that would have acted as a draw for game animals and the Paleoindian hunters who pursued them. Alternative explanations were forwarded to explain Paleoindian occupancy at Sandy Springs including the presence of a rare xeric ecosystem and location along an early trail system connecting the Cumberland, Tennessee, and Ohio river valleys. Geomorphological and geochronological research indicates that Sandy Springs is located on a late Quaternary landscape shaped by aeolian-alluvial processes which may have deposited sediments that presently cover archaeological deposits including Paleoindian components. Geomorphological analysis demonstrates that upper Ohio Valley Pleistocene landforms are commonly capped by Holocene aeolian sediments. Finally, research also suggests that paleoclimate, including the welldocumented 8.2 and 4.2 ka events, may have played a significant role in shaping landscapes in the upper Ohio Valley throughout the late Pleistocene and the Holocene. 


\section{TABLE OF CONTENTS}

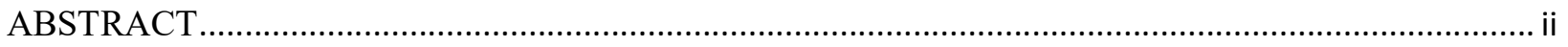

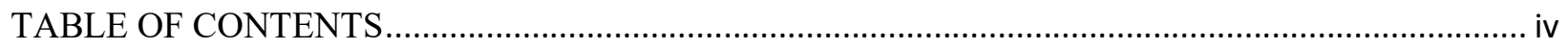

CHAPTER 1. INTRODUCTION ………………………….................................................

Dissertation and Chapter Organization ………………………………………………………...

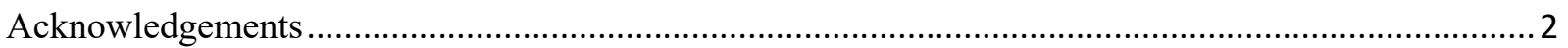

CHAPTER 2. GIS MODELING OF REGIONAL PALEOINDIAN LAND-USE BEHAVIOR IN SOUTHERN OHIO: A CROSS-REGIONAL AND CROSS-TEMPORAL APPROACH...........................5

Chapter 2. Introduction and Summary of Findings........................................................................ 5

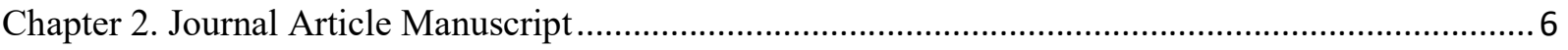

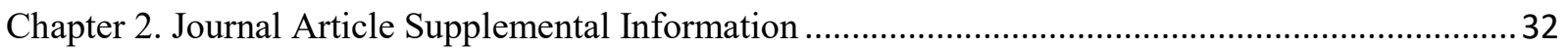

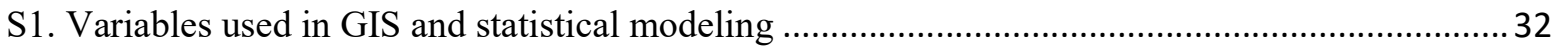

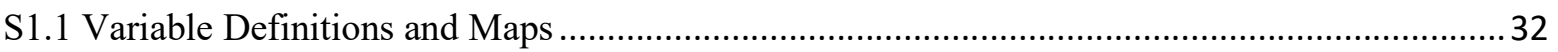

S1.2 Data results of statistical testing for Comprehensive, Cross-regional, and Cross-temporal GIS

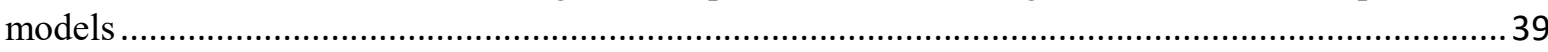

S1.3 Data results for the Binary Logistic Regression test...........................................................4

CHAPTER 3. RECONSIDERING THE POTENTIAL ROLE OF SALINE SPRINGS IN THE PALEOINDIAN OCCUPATION OF SANDY SPRINGS, ADAMS COUNTY, OHIO............................ 49

Chapter 3. Introduction and Summary of Findings............................................................................. 49

Chapter 3. Manuscript of Published Journal Article .......................................................................... 50

Chapter 3. Non-journal Article Supplemental Information ..................................................................68

CHAPTER 4. MIDWESTERN SAND DUNES, GEOARCHAEOLOGY, AND LIDAR: GEOMORPHIC LANDFORM ANALYSIS OF THE SANDY SPRINGS PALEOINDIAN SITE IN THE UPPER OHIO

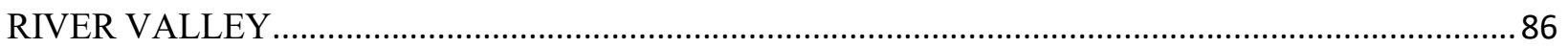

Chapter 4. Introduction and Summary of Findings.......................................................................... 86

Chapter 4. Journal Article Manuscript …………………………………………………………..... 87

Chapter 4. Journal Article Supplementary Material ....................................................................... 113

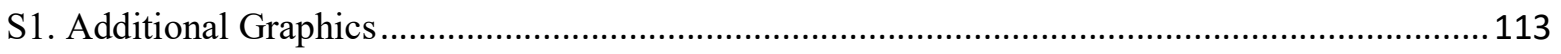

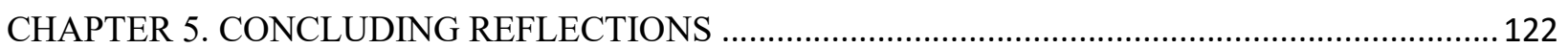




\section{CHAPTER 1. INTRODUCTION}

Despite Ohio's prominent role in the trajectory of archaeological research into the Native American Paleoindian period (13.5 to 11.4 ka years ago, after Miller and Gingerich, 2013), it has been over 30 years since the last attempt to model Paleoindian land-use behavior at a resolution that includes the southern half of the state (Seeman \& Prufer, 1982). The 1982 analysis relied on county-level densities of projectile points along with ancillary information on large sites to infer the nature of Paleoindian occupation. The aggregation of data at the county, state, or physiographic level to infer land-use behavior is common in Paleoindian studies, both in Ohio and elsewhere (Anderson, 1996; Anderson \& Gillam, 2000; Loebel, 2012; Shott, 2002). Although appropriate for suggesting broad regional trends, data aggregation has potential analytical issues related to the Modifiable Areal Unit Problem (Harris, 2006) and collector bias (Briggs; Buchanan, 2003). Aggregated data also are limited when modeling potential coassociations between environmental variables and archaeological site locations. Thus, a reliance on aggregated data risks missing potential fine-scale patterns that may exist in our datasets.

With these factors in mind, this dissertation revisits Paleoindian adaptation and land-use behavior in southern Ohio and by association the eastern United States (Purtill, 2015) (Figure 11-1). This is accomplished through a study of Paleoindian occupation at two distinct resolutions in southern Ohio: (i) regional; and, (ii) local, or site-level. Several factors favor a reanalysis at this time. First, continued research has increased the inventory of Paleoindian archaeological sites since 1982. Second, significant advances in the recording of archaeological and environmental datasets have occurred. For example, the Ohio Archaeological Inventory form has been expanded in scope, vetted, and is now in a digital format. These data can be represented as point data that allow the application of modeling and statistical methods. Finally, the rise of GISbased computing power with spatial analyst capabilities and increased use of spatial statistics provides new tools for exploring the distribution of archaeological sites.

This dissertation includes five chapters (numbered 1 through 5, including this Introduction) and one appendix (lettered A). Results of the regional component of this research are provided in Chapter 2. The regional level is explored through a GIS and statistical modeling approach of Paleoindian site locations against random environmental information to evaluate current theories of Paleoindian land-use behavior (e.g., Koldehoff \& Loebel, 2009; Lepper, 1986; Meltzer \& Smith, 1986; Kenneth B. Tankersley, 1990). Significant patterns are further explored through comparison of similarly generated trends for later archaeological sites dating to the Early Archaic ( 11-8.5 ka) and Late Archaic ( 6-2.7 ka) periods (Purtill, 2009). A binary logistical regression test is calculated with significant variables to provide a method to assess the relative strength of individual variables in explaining Paleoindian site location.

The second, site-level, component of this dissertation involves archaeological, geomorphological, and geochronological investigation of the Sandy Springs archaeological site (Chapman \& Otto, 1976; Cunningham, 1973; Seeman, Summers, Dowd, \& Morris, 1994). Results of this research are provided in Chapters 3 and 4. Research focuses on landscape reconstruction and geochronology with special emphasis on aeolian sand dune formation histories. Furthermore, proxy paleoenvironmental indicators are documented through investigation of sediments at Sandy Springs. Research focuses on potential explanations for site 
function during the Paleoindian period and directly tests the hypothesis that saline springs were a primary reason for Paleoindian occupation at the site. Chapter 5 provides some concluding statements and provides thoughts for future directions of Paleoindian research.

\section{Dissertation and Chapter Organization}

As proposed in Purtill (2015, p. 24), research results are provided in the form of three manuscripts suitable for submission to peer-reviewed journals. These manuscripts are the main body of text for Chapters 2 through 4. Chapter 3 was published in Journal of Archaeological Science, Reports during 2017 (Purtill, 2017) and the Chapters 2 and 4 manuscripts are ready for journal submission. Because each chapter and accompanying supplemental information were to be submitted as stand-alone articles, there is some formatting incongruity between chapters in this dissertation to meet the formatting rules of each journal. Aside from the Introduction (Chapter 1) and Conclusion (Chapter 5), each chapter has the following basic outline:

\section{Chapter Heading \\ 2. Introduction and Summary of Findings \\ 3. Journal Article \\ 4. Journal Supplemental Information \\ 5. Non-journal Supplemental Information}

The Chapter Heading provides the title for the chapter. The Introduction and Summary of Findings section provides a brief introduction to the journal article. Major research findings also are summarized in a series of bullet points. The Journal Article section includes the full text of the journal article as prepared for submission. In the case where the journal article has been published (Chapter 3), a copy of the published paper is provided in Appendix A. The references section is omitted from each individual chapter, but instead are combined in a comprehensive listing at the end of this dissertation. To facilitate a single references section, all in-text citations and references are formatted using the APA $6^{\text {th }}$ edition style.

The Journal Supplemental Information section includes research data to be included as online supplemental information and includes various data tables, additional maps, variable definitions, method discussions, etc. The Non-journal Supplemental Information section includes newly generated data not incorporated into prepared manuscripts due to concerns with article length or in response to reviewer comments. Since this information was generated during the course of investigation, it was included as appendix data to provide a record of these findings. It is anticipated that some of the non-article supplemental information will be incorporated into future journal articles.

\section{Acknowledgements}

Geochronology and micromorphology work is supported by a 2016 NASA West Virginia Space Grant Consortium Graduate Research Fellowship and a 2015 West Virginia University Eberly College of Arts and Sciences Doctoral Student Research Program grant. Various individuals 
provided project assistance including Steve Baker of USDA-NRCS; Mike Angle, Frank Fugitt, and Nathan Erber of the Ohio Geological Survey; James Thompson and Kathy Benison of West Virginia University; Chris Bedel of the Edge of Appalachia Preserve System; and Nancy Stranahan of the Arc of Appalachia. William Kennedy of the Dayton Society of Natural History also provided GIS assistance. Special thanks go to the Adams, Rutledge, and Whisman families for providing property access during fieldwork. The following West Virginia University students and volunteers also assisted in field and laboratory efforts: Francesca Basil, Andrew Braun, Nick Dadamo, Sara DeAloia, Nancy Gostic, Zach Haidar, Heather Jewel, Matt Koerner, Miles Reed, Emily Swaney, Katie Wasley, and Joey Zampayo.

Members of my Ph.D. committee were helpful and generous with their time including Jamison Conley (West Virginia University), Trevor M. Harris (West Virginia University), J. Steven Kite (West Virginia University), Mark F. Seeman (Kent State University), and James Thompson (West Virginia University). J. Steven Kite, committee chair, deserves special thanks for guiding this project from start to finish. 


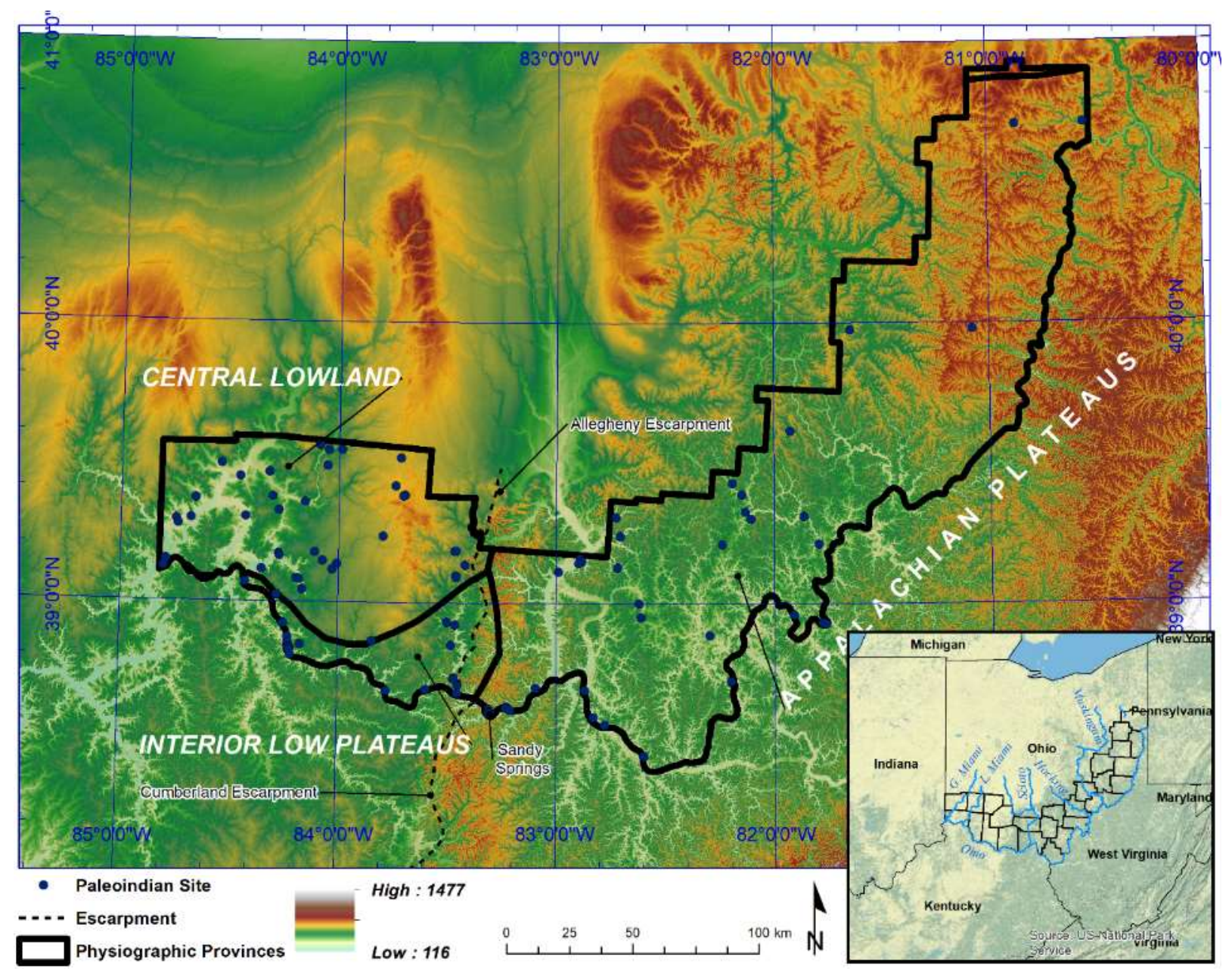

Figure 1-1. Shaded relief topographic map showing study area in southern Ohio defined by physiographic boundaries (main map) and county boundaries and major rivers (inset map). Ohio Archaeological Inventory Paleoindian site database also illustrated. Elevations presented in meters above mean sea level. 
CHAPTER 2. GIS MODELING OF REGIONAL PALEOINDIAN LAND-USE BEHAVIOR IN SOUTHERN OHIO: A CROSS-REGIONAL AND CROSS-TEMPORAL APPROACH

\section{Chapter 2. Introduction and Summary of Findings}

Purtill (2015) proposed that the first dissertation chapter conduct GIS and statistical modeling of Paleoindian (13.5 to 11.4 ka years ago, after Miller and Gingerich, 2013) site locations against random environmental data. This approach is used to evaluate several current theories of Paleoindian land-use behavior in the eastern U.S. (e.g., Koldehoff \& Loebel, 2009; Lepper, 1986; Meltzer \& Smith, 1986; Kenneth B. Tankersley, 1990). Significant patterns are further explored through comparison of similarly generated trends for later archaeological sites dating to the Early Archaic ( 11-8.5 ka) and Late Archaic ( 6-2.7 ka) periods (Purtill, 2009). Finally, a binary logistical regression test is calculated to provide a way to assess the relative strength of individual variables in explaining Paleoindian site locations. The primary findings of this research are as follows:

- Study results broadly indicate that Paleoindians did not practice a uniform land-use strategy in the upper Ohio Valley but instead adapted strategies to fit local conditions.

- A potentially distinct Paleoindian land-use strategy is identified for the Interior Low Plateaus province of Ohio. This strategy may reflect strengthening post-11 ka interaction for groups occupying the Cumberland, Tennessee, and upper Ohio drainages. Overrepresentation of Paleoindian sites on Mollisols and karst landscapes may suggest a preference for prairie settings.

- An absence of Paleoindian sites in the lower Scioto River is interpreted as deliberate avoidance of the lower section of the valley. The cause of this avoidance is unclear but a contributing factor may be environmental conditions that favored increased flooding and eolian deflation of braid bars and alluvial plains resulting in an inhospitable environment for human occupation or travel. Such conditions may have resulted in habitat loss and fragmentation and a general decrease in species diversity for the valley. Relatedly, an overabundance of Paleoindian sites in the Ohio Brush Creek valley may indicate a less impacted north-south travel corridor connecting unglaciated and glaciated landscapes.

- The combined modeling of Paleoindian, Early Archaic, and Late Archaic site distributions provides a method to assess the potential of collector bias when interpreting spatial distributions of archaeological data. Results indicate that modern population densities are positively correlated with archaeological site densities for all tested subperiods. This result may indicate that suitable sites remain suitable sites for occupation throughout time. Results also indicate that collector bias is not restricted to Paleoindian assemblages but likely impacts all archeological assemblages. Cross-temporal approaches provide a means to mitigate the influence of such bias on the archaeological record. 
Chapter 2. Journal Article Manuscript

Status: to be submitted to Journal of Archaeological Science

\title{
GIS and Statistical Modeling of Eastern U.S. Paleoindian Land-Use Strategies: A Comprehensive, Cross-regional, and Cross-temporal Approach in the Upper Ohio River Valley
}

\section{Corresponding author}

Matthew P. Purtill

\begin{abstract}
How eastern U.S. Paleoindians (13.5 to 11.4 ka years ago) utilized and interacted with their surrounding landscapes provides an alternative means to examine socio-economic aspects of late Pleistocene foragers apart from the more common focus on toolstone procurement and organization. Various purported co-associations between Paleoindian site locations and environmental settings or topographic positions have been advocated in the literature, yet few have been empirically tested to determine their accuracy or regional exclusivity. Since many coassociations have been incorporated into archaeological theories, assessing their validity is critical. In this study, combined GIS and statistical modeling were used to test the accuracy of some of the most commonly suggested co-associations in the eastern U.S. Paleoindian literature. An environmental background dataset for southern Ohio in the upper Ohio Valley was defined and compared to archaeological site information. Global, cross-regional and cross-temporal modeling was used to determine if verified site location trends were (i) regionally isolated or universal across the study area, (ii) exclusive to a Paleoindian pattern or part of a more common forager condition, and (iii) better able to control for the negative effects of collector bias in interpreting the archaeological record. Study results broadly indicate that Paleoindians did not practice a uniform land-use strategy in the upper Ohio Valley, but instead adapted strategies to fit local conditions. Study results also indicate a potentially distinct land-use strategy for the Interior Low Plateaus that may reflect strengthening post-11 ka interaction for groups occupying the Cumberland, Tennessee, and upper Ohio drainages. An absence of Paleoindian sites in the lower Scioto River is interpreted as deliberate avoidance of this glacial meltwater and outwash impacted valley due to threat of flooding and environmental pollution issues. Finally, a methodological approach is proposed that includes modeling Early and Late Archaic sites to constrain the negative effects of collector bias when interpreting Paleoindian site distributions. Since collector bias has long been cited as a limiting factor in Paleoindian distributional studies, cross-temporal approaches as applied here provides one way to mitigate the influence of such bias on the archaeological record.
\end{abstract}

\section{Keywords}

Paleoindian land-use behavior

GIS and statistical modeling

Upper Ohio River Valley

Eastern U.S. Paleoindians 


\section{Introduction}

In the eastern U.S., the degree to which variation existed in Paleoindian (13.5 to 11.4 ka years ago, after Miller and Gingerich, 2013) material culture, settlement systems, band size and composition, and subsistence strategies, remains debated. Although traditional views posited a continent-wide, high mobility hunting adaptation, especially for the initial Clovis colonization (Briggs Buchanan \& Collard, 2010; C. V. Haynes, 1964; Hemmings, 2004; Kelly \& Todd, 1988; R. J. Mason, 1962; Sholts, Stanford, Flores, \& Wärmländer, 2012), most now acknowledge that the Mississippi River with its frequent late Pleistocene glacial meltwater pulses and high sediment loads (Rittenour, Blum, \& Goble, 2007; Rittenour, Goble, \& Blum, 2005) represented a natural border separating eastern from western Paleoindian settlement-subsistence patterns (Anderson \& Faught, 1998; Briggs Buchanan, Hamilton, Kilby, \& Gingerich, 2016, p. 118; Morrow, 2014). In the eastern U.S., recent research has focused are defining models of Paleoindian regional variability especially highlighting aspects of land-use behavior, subsistence strategy, social learning, or technological organization (Anderson, 1990, 1995, 1996; Anderson \& Gillam, 2000; Broster, Norton, Miller, Tune, \& Baker, 2013; Briggs Buchanan et al., 2016; Briggs Buchanan, O’Brien, \& Collard, 2014; Cannon \& Meltzer, 2004, 2008; Eren et al., 2016; Eren \& Desjardine, 2015; Lepper, 1988; Lepper \& Meltzer, 1991; Loebel, 2012; Lothrop, Lowery, Spiess, \& Ellis, 2016; Meltzer, 1985, 1988, 2009, p. 286; Meltzer \& Smith, 1986; G. L. Miller et al., 2018; Seeman, 1994; Smallwood, 2012; Speth, Newlander, White, Lemke, \& Anderson, 2013, p. 112).

Embedded within many of these land-use discussions are various assertions for the coassociation of archaeological site location and environmental settings or topographic positions (Table 2-1). Assuming they are valid, purported co-associations imply that certain positions on the landscape acted either as draws for foragers or triggered avoidance behavior in groups during the course of annual settlement rounds or establishment of home territories. Importantly, many hypothesized co-associations have been critical in the development of eastern U.S. Paleoindian theory and land-use models, especially as they relate to development of social networks, technological organization and evolution, and prey choice (e.g., Eren, Buchanan, \& O'Brien, 2015; Shott, 2004). Many suggested co-associations, however, are based on simple qualitative impressions and have not been empirically tested or verified which leaves their accuracy, and any generalizations on which they are based, in doubt. 
Table 2-1. Some suggested environmental settings of interest to Eastern U.S. Paleoindians

\begin{tabular}{|c|c|c|c|}
\hline $\begin{array}{l}\text { Desired } \\
\text { Environmental } \\
\text { Setting }\end{array}$ & $\begin{array}{l}\text { Perceived Benefit or } \\
\text { Impact }\end{array}$ & $\begin{array}{l}\text { Archaeological } \\
\text { Correlate }\end{array}$ & Key References \\
\hline karst landscape & $\begin{array}{l}\text { presence of prairie } \\
\text { ecotones and associated } \\
\text { game such as bison, } \\
\text { high-quality chert }\end{array}$ & $\begin{array}{l}\text { over-representation of } \\
\text { sites in karst geology }\end{array}$ & $\begin{array}{l}\text { Anderson \& Faught, 1998, p. 175; Broster, Norton, } \\
\text { Miller, Tune, \& Baker, 2013, p. 309; Gardner, 1974; } \\
\text { Pevny, Thulman, \& Faught, 2018, p. 222; Seeman, } \\
\text { Summers, Dowd, \& Morris, 1994, p. 84; Smith, } \\
1990\end{array}$ \\
\hline $\begin{array}{l}\text { major river } \\
\text { drainage }\end{array}$ & $\begin{array}{l}\text { travel corridors, areas } \\
\text { that provide good views } \\
\text { of game or concentrate } \\
\text { game }\end{array}$ & $\begin{array}{l}\text { closer than average } \\
\text { proximity of sites to } \\
\text { major drainages }\end{array}$ & $\begin{array}{l}\text { Anderson, 1990, 1995, pp. 13-15, 1996, p. 159; } \\
\text { Anderson \& Faught, 1998, p. 175; Anderson \& } \\
\text { Gillam, 2000; Holliday \& Miller, 2014, pp. 228- } \\
\text { 233; Jackson, 1990, pp. 280-281; Jodry, 2005; } \\
\text { Prufer \& Baby, 1963, p. 24; Seeman \& Prufer, 1982, } \\
\text { pp. 159-160; Smith, 1990, p. 244; Tune, 2016, p. } \\
\text { 311 }\end{array}$ \\
\hline $\begin{array}{l}\text { major drainage } \\
\text { confluence areas }\end{array}$ & $\begin{array}{l}\text { aggregation loci, areas } \\
\text { that concentrate game }\end{array}$ & $\begin{array}{l}\text { closer than average } \\
\text { proximity of sites to } \\
\text { major drainage } \\
\text { confluence areas }\end{array}$ & $\begin{array}{l}\text { Anderson, 1995, p. 15, 1996, p. 52; Seeman \& } \\
\text { Prufer, 1982, p. 160; Shane Miller, 2016; } \\
\text { Tankersley, 1996, p. 37; Tune, 2016, p. } 311 \text { (cf. } \\
\text { Cochran, Richey, \& Maust, 1990; Lepper, 1986, p. } \\
\text { 280) }\end{array}$ \\
\hline wetlands or lakes & $\begin{array}{l}\text { unique plant-animal } \\
\text { communities such as } \\
\text { migratory waterfowl, } \\
\text { potable water, travel }\end{array}$ & $\begin{array}{l}\text { closer than average } \\
\text { proximity of sites to } \\
\text { wetland or lake settings }\end{array}$ & $\begin{array}{l}\text { Boisvert, 2012; Ellis, 2011; Hemmings, 2004; } \\
\text { Loebel, 2012; McWeeney \& McWeeney J., 2013; } \\
\text { Pevny, Thulman, \& Faught, 2018; F. W. Robinson, } \\
\text { Crock, \& Dorshow, } 2018 \text { (cf. Prufer \& Baby, 1963, } \\
\text { p. 62; Seeman \& Prufer, 1982, p. 161) }\end{array}$ \\
\hline $\begin{array}{l}\text { overlooks or high } \\
\text { elevations }\end{array}$ & $\begin{array}{l}\text { broad viewshed of } \\
\text { surrounding area, } \\
\text { especially important for } \\
\text { game monitoring }\end{array}$ & $\begin{array}{l}\text { topographic positions } \\
\text { affording larger than } \\
\text { average viewsheds of } \\
\text { surrounding terrain }\end{array}$ & $\begin{array}{l}\text { Anderson, 1995, p. 15; Freeman, Smith, \& } \\
\text { Tankersley, 1996, p. 402; Holliday \& Miller, 2014, } \\
\text { p. 235; Jackson, 1990, pp. 280-281; Lepper, 1988, } \\
\text { p. 41; Seeman \& Prufer, 1982, pp. 159-160; } \\
\text { Seeman, Summers, Dowd, \& Morris, 1994, p. 83 } \\
\text { (cf. Broster, Norton, Miller, Tune, \& Baker, 2013, p. } \\
\text { 309; Cochran, Richey, \& Maust, 1990) }\end{array}$ \\
\hline $\begin{array}{l}\text { sandy ridges, } \\
\text { dune fields, } \\
\text { strandlines (often } \\
\text { associated with } \\
\text { wet areas) }\end{array}$ & $\begin{array}{l}\text { broad viewshed of } \\
\text { surrounding area, } \\
\text { unique plant-animal } \\
\text { communities, facilitate } \\
\text { travel through otherwise } \\
\text { swampy terrain }\end{array}$ & $\begin{array}{l}\text { over-representation of } \\
\text { sites on sandy soils or } \\
\text { sand dune/strandline } \\
\text { landforms }\end{array}$ & $\begin{array}{l}\text { Holliday \& Miller, 2014; Jackson, Ellis, Morgan, \& } \\
\text { McAndrews, 2000; Lothrop \& Bradley, 2012, pp. } \\
\text { 24-25; Lothrop, Lowery, Spiess, \& Ellis, 2016, p. } \\
203\end{array}$ \\
\hline $\begin{array}{l}\text { animal trails, } \\
\text { migration paths, } \\
\text { river corridors } \\
\text { used for } \\
\text { transportation }\end{array}$ & $\begin{array}{l}\text { proximity to herd game; } \\
\text { ready-made travel } \\
\text { corridors }\end{array}$ & $\begin{array}{l}\text { closer than average } \\
\text { distance of sites to } \\
\text { animal trails, migration } \\
\text { paths, or river corridors } \\
\text { used for transportation }\end{array}$ & $\begin{array}{l}\text { Anderson \& Gillam, 2000; Anderson, Smallwood, \& } \\
\text { Miller, 2015; Cunningham, 1973; Jackson, 1990, pp. } \\
\text { 133-138; O’Shea, Lemke, \& Reynolds, 2013; } \\
\text { Seeman \& Prufer, 1982, pp. 159-160; Shane Miller, } \\
\text { 2016; Smith, 1990, p. 244; Spiess, Cowie, \& } \\
\text { Bartone, 2012 }\end{array}$ \\
\hline $\begin{array}{l}\text { Avoidance of } \\
\text { rugged terrain or } \\
\text { variable use of } \\
\text { physiographic } \\
\text { regions }\end{array}$ & $\begin{array}{l}\text { avoidance of areas not } \\
\text { conducive to big-game } \\
\text { hunting, travel, or } \\
\text { availability of variable } \\
\text { ecotones and associated } \\
\text { resources }\end{array}$ & $\begin{array}{l}\text { under-representation of } \\
\text { sites in areas } \\
\text { characterized by rugged } \\
\text { terrain }\end{array}$ & $\begin{array}{l}\text { Anderson \& Faught, 1998, p. 175; Lane \& } \\
\text { Anderson, 2001; Lepper, 1988; Meltzer, 1985; } \\
\text { Prufer \& Baby, 1963, pp. 62-63; Ritchie, 1965, pp. } \\
\text { 4-5; Seeman \& Prufer, 1982; Tankersley, Smith, \& } \\
\text { Cochran, } 1990\end{array}$ \\
\hline $\begin{array}{l}\text { Southern to } \\
\text { southwestern } \\
\text { exposures }\end{array}$ & maximize warmth & $\begin{array}{l}\text { over-representation of } \\
\text { sites with south- to } \\
\text { southwest-facing } \\
\text { aspects }\end{array}$ & Lepper, 1988, p. 41; Lepper \& Meltzer, 1991, p. 180 \\
\hline $\begin{array}{l}\text { Eastern U.S. tall- } \\
\text { grass prairie }\end{array}$ & $\begin{array}{l}\text { proximity to herd } \\
\text { animals or mega-fauna }\end{array}$ & $\begin{array}{l}\text { over-representation of } \\
\text { sites in environments } \\
\text { characterized by tall- } \\
\text { grass prairies, e.g., } \\
\text { presence of Mollisols }\end{array}$ & $\begin{array}{l}\text { Johnson, Politis, Gutierrrez, Martinez, \& Miotti, } \\
\text { 2006; T. M. N. Lewis, } 1953\end{array}$ \\
\hline
\end{tabular}


To better determine if the environmental co-associations or variable land-use strategies listed in Table 2-1 can be verified empirically, this study explores land-use behavior of eastern U.S. Paleoindians through a Geographic Information Systems (GIS) and statistical modeling approach. Although traditionally used to inductively 'predict' site locations (Kvamme, 2006; Legg, Regis, Lambert, Liesch, \& Travis, 2017; Pitblado, Cannon, \& Fowler, 2011; Warren \& Asch, 2003) and despite its numerous operational and theoretical issues (Harris, 2006; G. R. Lock \& Harris, 2000, 2006), GIS modeling can validate or refine existing theories and propose new models of past land-use behavior (Duke \& King, 2014; Loebel, 2012; Ridges, 2006). Landuse studies are especially vital for Paleoindian research because they provide an alternative method to examine aspects of decision making for late Pleistocene foragers unrelated to toolstone preference, procurement, and technological organization (Sillitoe \& Hardy, 2003; Speth et al., 2013).

To conduct this study, a database was assembled from southern Ohio in the upper Ohio River Valley of the eastern U.S. (Figure ). This area is ideal to evaluate models of eastern U.S. Paleoindian land-use strategies for several reasons. This reach has been recognized as yielding a robust inventory of Paleoindian components, specifically fluted bifaces (Briggs; Buchanan, 2003; R. J. Mason, 1962; D. S. Miller et al., 2013; Prasciunas, 2011; Prufer \& Baby, 1963; Seeman \& Prufer, 1982) which led Anderson to suggest that this valley was among the initial staging areas for Clovis colonization in the east (Anderson, 1990, 1995, 1996; Anderson \& Faught, 1998; Eren et al., 2016). Southern Ohio also includes both low-relief, glaciated, and high-relief, unglaciated landforms. A dense river network afforded Pleistocene foragers broad habitat selectivity as various biomes and associated resources would have been readily accessible within a few day's journey. It is suggested that this regional interconnectedness allowed Paleoindian land-use decision making in southern Ohio to be flexible and not overly conditioned by environmental necessity as may have been the case for groups in more homogenous, low biomass landscapes (e.g., late Pleistocene upper U.S. northeast). In other words, Paleoindians operating in southern Ohio had some opportunity to select from distinct habitats when moving through the landscape.

\section{Environmental and Climatic Background}

To model eastern U.S. Paleoindian land-use in the upper Ohio River Valley, a study area was defined in southern Ohio that includes 26 southern counties proximal to the Ohio River (Adams, Athens, Belmont, Brown, Butler, Carroll, Clermont, Clinton, Columbiana, Gallia, Guernsey, Hamilton, Harrison, Highland, Jackson, Jefferson, Lawrence, Meigs, Monroe, Morgan, Noble, Pike, Scioto, Vinton, Warren, and Washington) (Figure 2-1). The study area extends between $38.403416^{\circ} \mathrm{N}$ and $40.930192^{\circ} \mathrm{N}$ and between $80.518895^{\circ} \mathrm{W}$ and $84.820300^{\circ} \mathrm{W}$. This represents $\sim 53,580 \mathrm{~km}^{2}$ of land situated within parts of three physiographic provinces: Appalachian Plateaus; Central Lowlands; and Interior Low Plateaus (Brockman, 2006; Coogan, 1996). As defined herein, southern Ohio contains heterogeneous landscapes characterized by low-relief, glaciated landforms to the west in the Central Lowlands to more heavily dissected, unglaciated high-relief landforms to the east in the Interior Low Plateaus and Appalachian Plateaus. Karst features are common in the Interior Low Plateaus and loess and cover sands mantle topographic highlands throughout the upper Ohio Valley (Aden, Pavey, Jones, \& Angle, 2012; Chappell, 
1988; Purtill \& Kite, 2015; Rutledge, Holowychuk, Hall, \& Wilding, 1975; Simard, 1989). Landform variability primarily relates to spatial differences in bedrock geology, relief, and glacial history. These factors, in turn, have influenced regional soils and biotic communities especially vegetation communities (Forsyth, 1970). Zonal climate patterns also vary by physiographic province, both today (Schmidlin, 1996) as well as in the past (Shane, 1994; Shane, Snyder, \& Anderson, 2001).

The study area is within the Ohio River watershed and is drained by numerous south-flowing tributaries. Major tributaries with drainage areas $>2500 \mathrm{~km}^{2}$ include the Little Beaver, Muskingum, Hocking, Scioto, Little Beaver, Little Miami, Great Miami, Mahoning, Muskingum, Paint Creek, Tuscarawas, and Whitewater rivers (Childress, 2001) (see Figure ). Alluvial landforms within these drainages largely consist of fine overbank sediments over coarser glaciofluvial and reworked basal channel deposits (Ray, 1974). Sediments derive from various sources including Wisconsin or Illinoian glacial outwash and lacustrine (Jacobson, Elston, \& Heaton, 1988; Kempton \& Goldthwait, 1959; Pavey et al., 1999). Outwash deposits entered the upper Ohio Valley through several outlets, including the Allegheny, Beaver, Little Beaver, Muskingum, Hocking, Scioto, Little Miami, Great Miami, and Whitewater rivers.

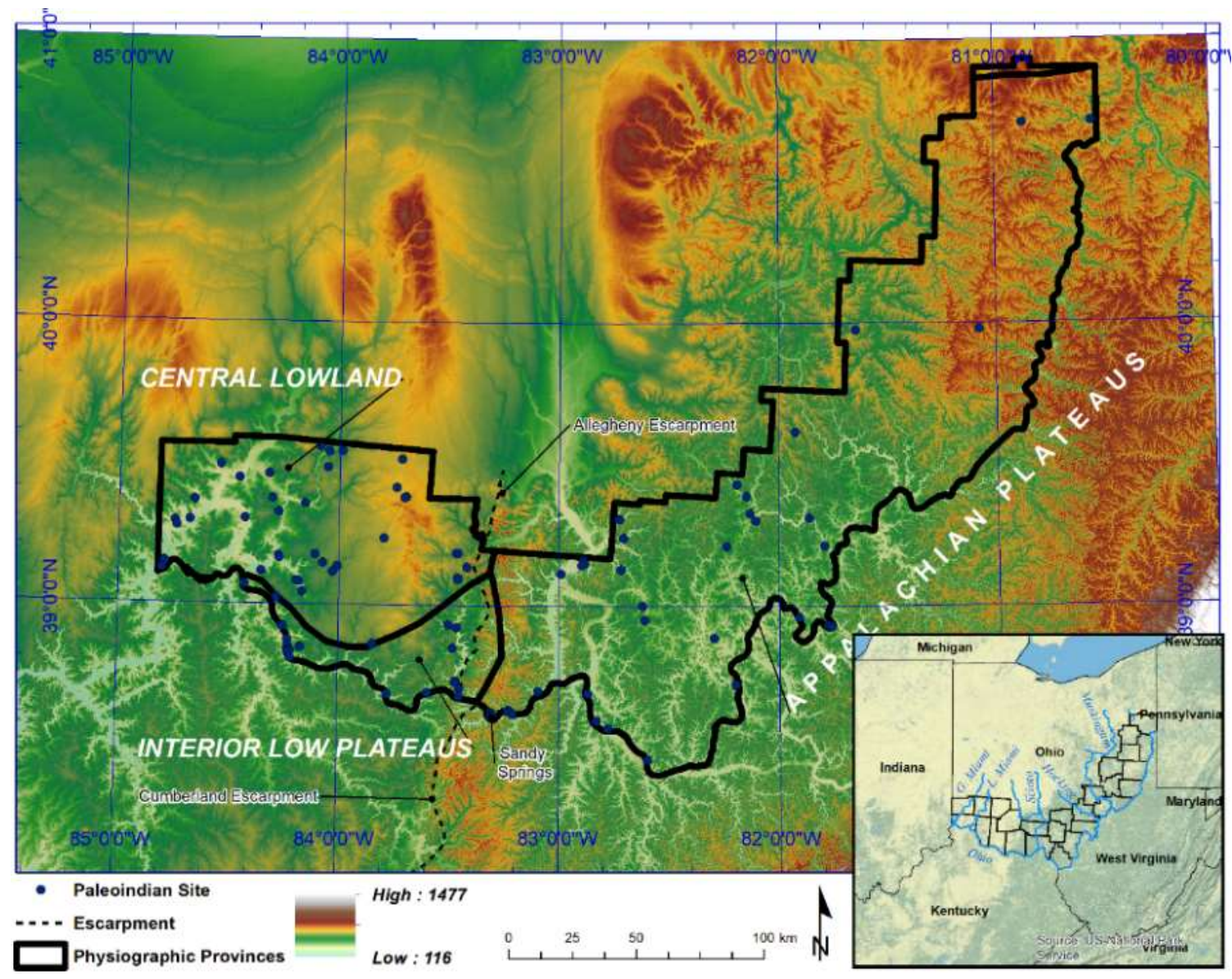

Figure 2-1. Shaded relief topographic map showing study area in southern Ohio defined by physiographic boundaries (main map) and county boundaries and major rivers (inset map). Ohio Archaeological Inventory Paleoindian site database also illustrated. Elevations presented in meters above mean sea level. 
The release of substantial glaciofluvial outwash into the upper Ohio River resulted in channel aggradation within a braided system during the late Pleistocene. The transition from a braided to single-thread system commonly is placed between 20 and $12 \mathrm{ka}$ in association with reduced transportation of outwash material (Mandel, 1988; Purtill, 2012; L. L. Ray, 1974; K. Tankersley, Munson, \& Tankersley, 1983). This timing closely correlates with estimates made for other eastern U.S. rivers including the upper Mississippi (Bettis, Benn, \& Hajic, 2008), the lower Mississippi (Kidder, Adelsberger, Arco, \& Schilling, 2008), and non-glacial coastal rivers in Georgia and the Carolinas (Leigh, 2006, 2008; Leigh, Srivastava, \& Brook, 2004). Research by Rogers (1990) near Apple Grove, West Virginia, suggests that the transition to a single-thread system may have occurred closer to $8.5 \mathrm{ka}$ for at least some reaches of the upper Ohio River.

Pollen data suggest time-transgressive, strong zonal variation in climate, vegetation, and precipitation trends across the study area between 13 and $8 \mathrm{ka}$ (Shane, 1994). During this time in the Central Lowlands, vegetation regimes transitioned quickly from a Picea-dominated parkland to an open Quercus-Carya-Fraxinus mixed deciduous forest. In higher, more rugged terrain such as the Appalachian Plateaus or Interior Low Plateaus, forests remained more diverse with a persistence of Picea and a closed canopy (Shane, 1994). An east-west zonal climate gradient existed throughout the entire 5000 year period with more dry, warm temperature seasonality to the east and wetter, more moderated seasonal extremes to the west.

The impact of the Younger Dryas Chronozone (YDC) between 12.9 and $11.6 \mathrm{ka}$ (Shuman et al., 2002) is difficult to fully assess in the study area since its impact appears locally variable (Meltzer \& Holliday, 2010; Yu, 2000). YDC appears to have strengthened patterns already in place with cooling in the Central Lowlands suggested by a 10-30\% increase in Picea pollen. In contrast, orographic factors appear to have moderated YDC climatic change in the Appalachian Plateaus region with only gradual change indicated (Shane, 1994, pp. 12-13). Reconstructed average January temperatures for the Appalachian Plateaus suggest warmer winters as the plateaus are suggested to be $12^{\circ} \mathrm{C}$ warmer than the Central Lowlands during cold months (Meltzer \& Holliday, 2010, pp. 5-8; Yu, 2000). By 8 ka in the study area, Quercus-Carya species dominate forest composition and climatic ranges and gradients assumed modern conditions (Halligan, 2013, pp. 63-65; Shane, 1994, pp. 13-14).

\section{Methods}

\subsection{Modeling structure}

To reveal patterning in Paleoindian land-use behavior, this study includes three stages of analysis. Initially, a 'comprehensive model' is constructed that compares the distribution of Paleoindian sites for the entire project area against the natural environmental background, defined through a series of random 'non-archaeological' points (see Section 3.2). Analysis of the comprehensive model will help determine potential patterning in site distributions and determine if site environmental factors statistically deviate from an assumption of randomness. The next step includes physiographic partitioning of Paleoindian and environmental datasets into three local datasets by province (Appalachian Plateaus, Interior Low Plateaus, and Central Lowlands). This 'cross-regional model' investigates the degree to which Paleoindians adapted their land-use 
behavior to match varying environmental settings and contexts (Prufer \& Baby, 1963; Seeman \& Prufer, 1982; Kenneth B. Tankersley \& Isaac, 1990).

Finally, a 'cross-temporal model' is constructed to compare Paleoindian data at the comprehensive level against two newly created datasets representing later foraging societies (Early and Late Archaic sites). This model stage seeks to determine if Paleoindian land-use differs significantly from the subsequent Archaic patterns and to isolate potential land-use foraging behaviors that are uniquely Paleoindian (see Gingerich, 2018, p. 417; Pevny, Thulman, \& Faught, 2018). It should be noted that, for this study, the appellation 'Paleoindian' includes all material dating between 13.5 and $11.4 \mathrm{ka} \mathrm{(after} \mathrm{D.} \mathrm{S.} \mathrm{Miller} \mathrm{et} \mathrm{al.,} \mathrm{2013).} \mathrm{The} \mathrm{succeeding}$ Archaic period in the Upper Ohio Valley is marked by the complete adoption of side- and corner-notched projectile points with a starting date sometime after $11.4 \mathrm{ka}$. Following Purtill (2009), the Early Archaic terminus is set at $8.45 \mathrm{ka}$ and the Late Archaic period dates between 5.95 and $2.65 \mathrm{ka}$. Middle Archaic assemblages are excluded in this study due to their poor archaeological representation perhaps reflecting a period of "substantial population reduction and relocation" (Purtill, 2009, p. 582).

\subsection{Archaeological components and environmental dataset}

Archaeological data was obtained from the Ohio Historical Preservation Office, Ohio Archaeological Inventory in 2014, and included Paleoindian ( $\mathrm{n}=100)$, Early Archaic $(\mathrm{n}=619)$, and Late Archaic $(\mathrm{n}=945)$ components (Figure 2-2). As discussed in the Supplemental Information, Paleoindian sites were vetted by the author prior to modeling. Although modest, the sample size of 100 Paleoindian sites compares favorably to other GIS studies of contemporary assemblages (Legg et al., 2017; Pitblado et al., 2011). Background environmental information was compiled through extraction of data from 702 random points generated using ArcMap's Random Point Generator tool. Tool parameters were set to evenly distribute random points across the 26-county study area. The 702 value mirrors the approach of Warren and Asch (2003, pp. 13-18) who restricted the number of random points in their study to seven times that of their test data to minimize the potential of committing Type I statistical errors.

\subsection{Assessing Collector Bias}

The potential influence of modern sampling biases in archaeological site distribution studies has been widely considered in Paleoindian research (Anderson \& Faught, 2000, p. 509; Blackmar, 2001, pp. 75-76; Briggs; Buchanan, 2003; Dorwin, 1966, p. 148; Lepper, 1983, 1985; Loebel, 2012; R. J. Mason, 1962, p. 235; Prasciunas, 2011; Seeman \& Prufer, 1984, 1982, p. 160, Shott, $2002,2004)$. Although contradictory results are reported, most suggest biases such as modern population densities are positively correlated with archaeological site densities. For example, Shott's (2002) analysis, which included the current southern Ohio study area, suggested modern population density, not land-use, positively correlates to Paleoindian site distribution at the county level. 

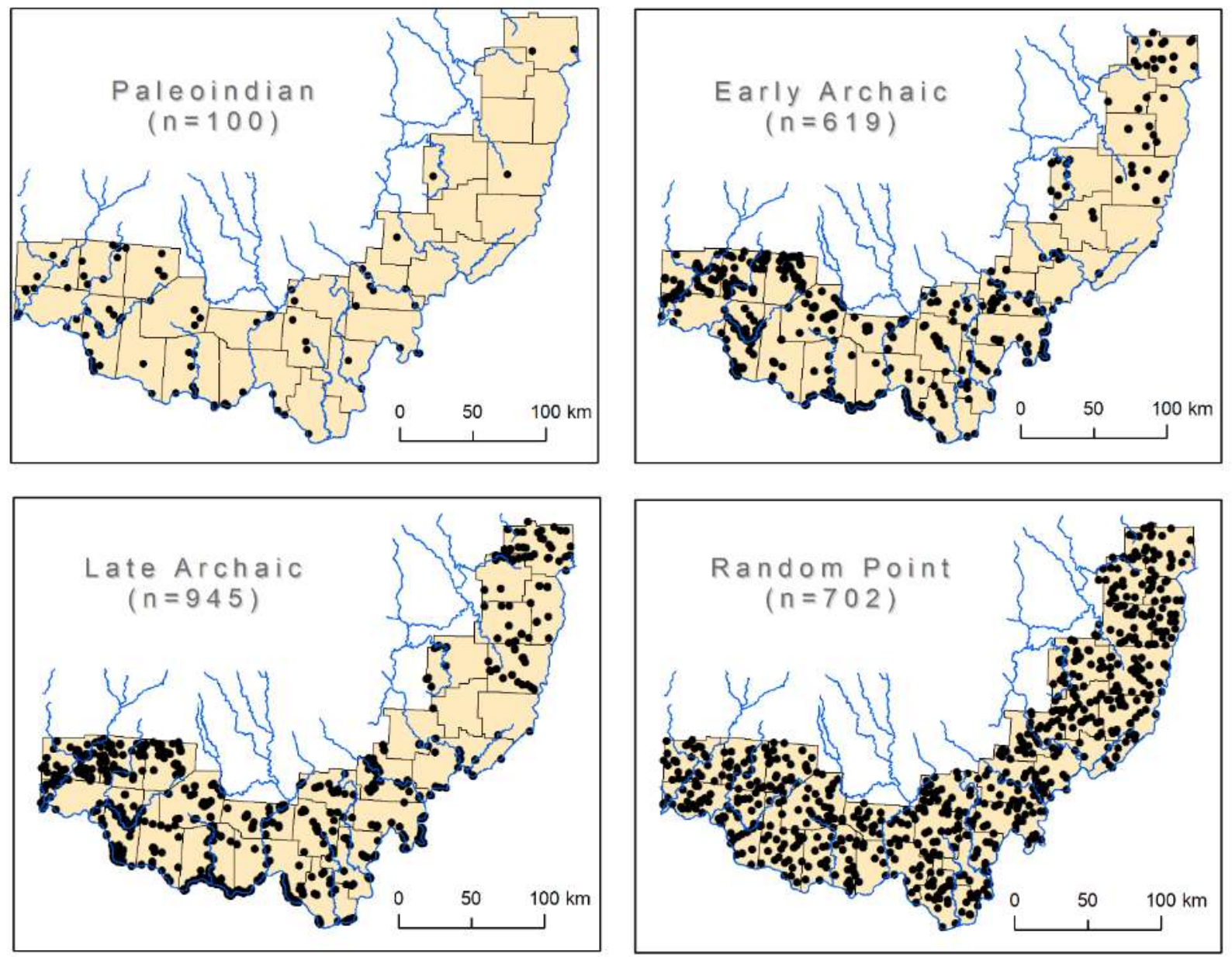

Figure 2-2. Distribution across the study area of archaeological sites and random points used in GIS modeling and statistical analysis. County boundaries and rivers also illustrated.

To evaluate the potential that modern population densities in southern Ohio also may bias study results, the distribution of archaeological sites is compared to modern population densities in the following manner. First, an inverse distance weighted (IDW) raster interpolation of 2013 population densities by census tract was constructed and reclassified into $10 \mathrm{~km}^{2}$ grid cells. Population values were extracted for both random points and archaeological sites and the resultant group means subject to a one-way ANOVA with Welch and Games-Howell post hoc tests.

\subsection{GIS data layers}

Table 2-2 provides an inventory of the 25 independent and 2 dependent variables used in modeling. Six independent categorical variables were dichotomized into dummy variables to assist in the statistical modeling process. Following dichotomization, modeling included 54 independent variables (Table 2-2). Additional information regarding variable source and definitions are provided in Supplemental Information. Variables were selected for this study due to their common use in archaeological modeling (Loebel, 2012; Pitblado et al., 2011; Ridges, 
2006; Warren \& Asch, 2003) and for their ability to evaluate the purported environmental coassociations with Paleoindian site distributions (see Table 2-1). Categorical variables were tested through Pearson's chi-square test $\left(\chi^{2}\right)$ with the Fisher's exact test employed for cells with fewer than 5 cases (Field, 2009, p. 690). Continuous variables were tested through comparison of means statistic. Since a calculated Kolmogorov-Smirnov (K-S) and Levene's statistic indicated a non-normal distribution with unequal variances for this dataset, the non-parametric MannWhitney test $(U)$ was selected for testing (Field, 2009, pp. 540-546). Finally, to quantify how influential individual variables were for site placement during the Paleoindian period, a binary logistical regression was calculated with independent variables found to be significant during the initial $\chi^{2}$ and $U$ tests in the comprehensive model. By inspecting the resultant odds ratio (OR) generated within the logistical regression output in SPSS, the potential effect that independent variables have on the odds of a specific geographic location containing a Paleoindian site can be assessed.

Table 2-2. Initial variable list to be used in the modeling process

\begin{tabular}{|c|c|c|}
\hline Variable & $\begin{array}{l}\text { Feature Type or Cell } \\
\text { Resolution }\end{array}$ & Variable Type \\
\hline \multicolumn{3}{|l|}{ Dependent } \\
\hline $\begin{array}{l}\text { Archaeological Site }(\text { Paleoindian }=100 ; \text { Early Archaic }=619 ; \text { Late } \\
\text { Archaic }=945)\end{array}$ & point & dichotomous \\
\hline Random Point $(\mathrm{n}=702)$ & point & dichotomous \\
\hline \multicolumn{3}{|l|}{ Independent } \\
\hline $\begin{array}{l}\text { Physiographic Province* (Appalachian Plateaus, Central Lowlands, } \\
\text { Interior Low Plateaus) }\end{array}$ & polygon & categorical \\
\hline $\operatorname{Aspect}^{*}(E, N, N E, N W, S, S E, S W, W$, flat $)$ & $150 \mathrm{~m}^{2}$ & categorical \\
\hline $\begin{array}{l}\text { Topographic Position Index (TPI), Fine Resolution* (flat, midslope, } \\
\text { ridge, toe slope, upper slope, valley) }\end{array}$ & $0.025 \mathrm{~km}^{2}$ & categorical \\
\hline TPI, Medium Resolution & $225 \mathrm{~km}^{2}$ & categorical \\
\hline TPI, Coarse Resolution & $300 \mathrm{~km}^{2}$ & categorical \\
\hline Soil Order* (Alfisols, Entisols, Inceptisols, Mollisols, Ultisols) & polygon & categorical \\
\hline Viewshed $\left(\mathrm{km}^{2}\right)$ & $150 \mathrm{~m}^{2}$ & continuous \\
\hline $\begin{array}{l}\text { Terrain Roughness Index (TRI), Fine Resolution (0-80, level; 81-116, } \\
\text { nearly level; 117-161, slightly rugged; 162-239, intermediately rugged; } \\
\text { 240-497, moderately rugged; 498-958, highly rugged; 959-4367, } \\
\text { extremely rugged) }\end{array}$ & $0.025 \mathrm{~km}^{2}$ & continuous \\
\hline TRI, Medium Resolution & $225 \mathrm{~km}^{2}$ & continuous \\
\hline TRI, Coarse Resolution & $300 \mathrm{~km}^{2}$ & continuous \\
\hline Landform Curvature, Fine Resolution & $0.025 \mathrm{~km}^{2}$ & continuous \\
\hline Landform Curvature, Medium Resolution & $225 \mathrm{~km}^{2}$ & continuous \\
\hline Landform Curvature, Coarse Resolution & $300 \mathrm{~km}^{2}$ & continuous \\
\hline Elevation $(\mathrm{m})$ & $10 \mathrm{~m}^{2}$ (1/3 arc-second $)$ & continuous \\
\hline Slope, Fine Resolution & $0.025 \mathrm{~km} 2$ & continuous \\
\hline Slope, Medium Resolution & $225 \mathrm{~km}^{2}$ & continuous \\
\hline Slope, Coarse Resolution & $300 \mathrm{~km}^{2}$ & continuous \\
\hline Distance to Large River (km) & polyline & continuous \\
\hline Distance to Medium River (km) & polyline & continuous \\
\hline Distance to Trails (km) & polyline & continuous \\
\hline Distance To Wetland $(\mathrm{km})$ & polygon & continuous \\
\hline Distance To Large River Convergence Pt. $(\mathrm{km})$ & point & continuous \\
\hline Distance To Large-Medium River Convergence Pt. (km) & point & continuous \\
\hline Distance to Karst Landscape $(\mathrm{km})$ & polygon & continuous \\
\hline Distance to Sand Dune $(\mathrm{km})$ & polygon & continuous \\
\hline
\end{tabular}

*denote categorical variables that were dichotomized to assist in the statistical modeling process. 


\subsection{Variable resolution}

The approach adopted here models scalable variables at three basic resolutions (fine, medium, and coarse). Although rare in archaeological research (cf. Delcourt, Delcourt, \& Davidson, 1983; Warren \& Asch, 2003), this scaled approach is common in ecological studies where multiple resolutions of a single variable are modeled to better explore the potential that significant relationships between species and environmental contexts exist, but are only detectable at specific scales (e.g., Ben Wu \& Smeins, 2000; Delcourt, Delcourt, \& Davidson, 1983; Denny \& Benedetti-Cecchi, 2012; Guisan, Weiss, \& Weiss, 1999; Weiss, 2001). Resolution selection should not be arbitrary but instead rooted in theoretical or empirical data. For this study, the forager/Paleoindian literature (Binford, 2001; Ellis, 2011; Kelly, 1995) was used to define certain scalable variables ('Slope', 'Landform Curvature', 'TRI', and 'TPI') to increase the potential that certain Paleoindian land-use behaviors can be detected through comparison of variables modeled at different resolutions. A fine resolution of $\sim 0.025 \mathrm{~km}^{2}$ was used to approximate near-site activity space and reflects the average size of Paleoindian sites in this database. A medium resolution of $\sim 225 \mathrm{~km}^{2}$ was selected as it closely matches ethnographic (Binford, 2001, pp. 234-239; Kelly, 1995, pp. 132-140) and Paleoindian archaeological data regarding daily resource exploitation zones (Smith, 1990; Kenneth B. Tankersley \& Isaac, 1990).

A coarse resolution for this study ideally would represent longer distance mobility patterns such as annual Paleoindian residential ranges. For Paleoindian societies, however, these ranges appear extensive (e.g., 10,000 km²; Ellis, 2011) and beyond the observational scale of southern Ohio. Initially, a coarse resolution of $800 \mathrm{~km}^{2}$ was used based on average area for thiessen polygons constructed about each Paleoindian site. Thiessen polygons, whose boundaries define the area closest to each site relative to all other sites, have been used to simulate hypothetical territorial boundaries or catchment zones (Lombardo \& Prümers, 2010; McCord \& Cochran, 2008; Nakoinz, 2010). This initial resolution, however, proved too coarse for the study area as it resulted in overly homogenized results. Since this dataset is overdispersed about the mean $\left(\mathrm{S}=1417.35 \mathrm{~km}^{2}\right)$, the median $\left(\sim 300 \mathrm{~km}^{2}\right)$ was selected to model the coarse resolution for this study.

\section{Results}

\subsection{Assessment of Collector Bias}

The first step in the modeling process was to assess the potential for collector bias in the distribution of archaeological sites. Extracted 2013 population means from cells for both random point $(\bar{x}=4569, s=6514.4$ ) and archaeological sites used in this study ([Paleoindian $\bar{x}=7269$, $s=8458.7$ ], [Early Archaic $\bar{x}=7245, s=8323.1$ ], [Late Archaic $\bar{x}=6812, s=7923$ ]) reveal significant between-group differences in a one-way ANOVA test $(F(3,438.6)=19.888, p=<.001)$. Specifically, sites from each temporal period are more likely to be located in densely populated grid cells than random points. Review of Games-Howell post hoc results, however, indicate that statistical differences only occur between archaeological sites and random points and not within archaeological subgroups. In other words, Paleoindian, Early Archaic, and Late Archaic sites all

tend to be located in grid cells characterized by high population densities, a result consistent with most previous studies (Briggs; Buchanan, 2003; Lepper, 1983; Shott, 2002), this correlation is 
equally as strong with Archaic sites. This finding illustrates the tacit, yet under-appreciated point that all archaeological datasets are biased to some degree in relation to modern population densities.
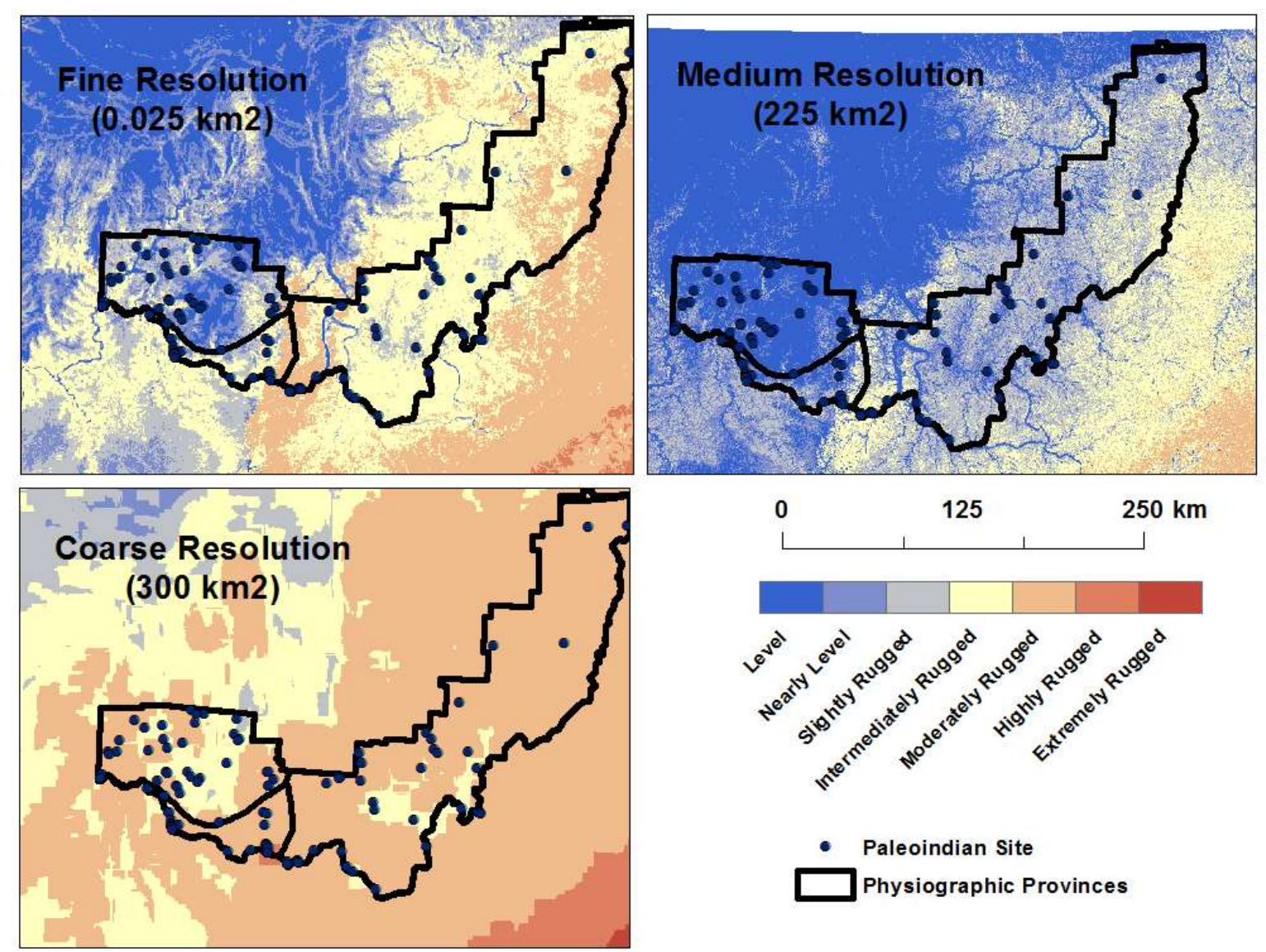

Figure 2. Example of TRI modeled at the three resolutions (fine, medium, coarse) used for this study. Paleoindian sites used for modeling and physiographic boundaries also illustrated. Note how adjustment of resolution impacts terrain roughness categories from fine to coarse resolution.

Even when acknowledged, there is no consensus regarding how to deal with sampling bias when interpreting Paleoindian spatial patterning. Several researchers note that, even for biased samples, we should not discount a priori the possibility that observed distributional patterns are valid (Briggs; Buchanan, 2003; Seeman \& Prufer, 1984, p. 228; Shott, 2002, p. 117). To partially mitigate the confounding effects of sampling bias in this study, Paleoindian site distributions were compared to Archaic site patterns. Simply put, it was reasoned that at every Early or Late Archaic find location there should have been a near-equal chance for recovery of Paleoindian material, if present, even if issues of preservation or visibility are considered (e.g., Holliday \& Miller, 2014, p. 222). This suggests that relative patterns of site densities between temporal periods should be valid and illustrative of potential chronological shifts in land-use behavior between Paleoindian and Archaic populations (see Purtill, 2009, for a similar approach). 


\subsection{Modeling}

The results of the GIS modeling and statistical analysis are summarized in Table 2-3 and Figure 3. Complete data results including mean and standard deviation values by variable are provided in the Supplemental Information (Tables S2-S5). Significant $(p<.05)$ variation between archaeological site locations and background environmental data (i.e., random point locations) was identified for numerous variables in the comprehensive, cross-regional, and cross-temporal models. Broad data trends are summarized below.

Table 2-3. Mean scores and relative proportions for Paleoindian sites per variable per physiographic province. Statistically significant associations $(\mathrm{p}<.05)$ between Paleoindian, Early Archaic, Late Archaic, and random point locations marked by letter superscript. Grey cells represent variables lacking statistically significant associations ( $>$ >.05).

\begin{tabular}{|c|c|c|c|c|c|}
\hline Variable & $\begin{array}{l}\text { Value } \\
\text { Represents }\end{array}$ & All Provinces & $\begin{array}{l}\text { Appalachian } \\
\text { Plateaus }\end{array}$ & Central Lowland & $\begin{array}{l}\text { Interior Low } \\
\text { Plateaus }\end{array}$ \\
\hline $\begin{array}{l}\text { Distance to Large } \\
\text { River Confluence } \\
\text { Pt. }\end{array}$ & $\mathrm{km}$ & $35.66^{\mathrm{a}}$ & $44.08^{\mathrm{a}}$ & 38.38 & $33.30^{\mathrm{a}}$ \\
\hline $\begin{array}{l}\text { Distance to Large- } \\
\text { Medium River } \\
\text { Confluence Pt. }\end{array}$ & $\mathrm{km}$ & $25.50^{\mathrm{a}}$ & 26.06 & $23.48^{\mathrm{a}}$ & 23.77 \\
\hline $\begin{array}{l}\text { Distance to Large } \\
\text { River }\end{array}$ & $\mathrm{km}$ & $7.31^{\mathbf{a}, \mathbf{b}, \mathbf{d}}$ & $8.84^{\mathrm{a}}$ & $10.43^{a}$ & $4.54^{\mathrm{a}}$ \\
\hline $\begin{array}{l}\text { Distance to } \\
\text { Medium River }\end{array}$ & $\mathrm{km}$ & 14.36 & 16.43 & 13.23 & 12.71 \\
\hline $\begin{array}{l}\text { Distance to } \\
\text { Wetland }\end{array}$ & $\mathrm{km}$ & $0.34^{\mathrm{a}, \mathrm{c}}$ & $0.42^{\mathrm{a}}$ & $0.32^{\mathbf{a}, \mathbf{d}}$ & $0.22^{\mathrm{a}}$ \\
\hline Distance to Trail & $\mathrm{km}$ & $1.99^{\mathrm{a}, \mathrm{c}}$ & $1.63^{\mathrm{a}}$ & $2.91^{\mathbf{a}, \mathbf{b}, \mathbf{d}}$ & $1.07^{\mathrm{a}}$ \\
\hline $\begin{array}{l}\text { Distance to Sand } \\
\text { Dune }\end{array}$ & $\mathrm{km}$ & 67.84 & $37.30^{\mathrm{a}}$ & $102.87^{\mathrm{a}}$ & $61.74^{\mathrm{a}}$ \\
\hline TRI, Fine Scale & scale & $57.5^{\mathrm{a}}$ & $55.6^{\mathrm{a}, \mathrm{b}}$ & $57.1^{\mathrm{a}}$ & $61.5^{\mathrm{a}}$ \\
\hline $\begin{array}{l}\text { TRI, Medium } \\
\text { Scale }\end{array}$ & scale & $137.9^{\text {a,d }}$ & $141.1^{a}$ & $122.4^{\mathrm{a}}$ & $158.2^{\mathrm{a}}$ \\
\hline TRI, Coarse Scale & scale & $283.6^{\mathrm{a}, \mathrm{d}}$ & 294.1 & $248.6^{a}$ & 323.4 \\
\hline $\begin{array}{l}\text { Landform } \\
\text { Curvature, Fine }\end{array}$ & scale & $3.0^{\mathbf{b}, \mathbf{c}}$ & 0.4 & $4.9^{\mathbf{b}, \mathbf{c}}$ & 4.3 \\
\hline $\begin{array}{l}\text { Landform } \\
\text { Curvature, } \\
\text { Medium }\end{array}$ & scale & $\begin{array}{l}-4.3 \\
\end{array}$ & -4.6 & -0.6 & -10.0 \\
\hline $\begin{array}{l}\text { Landform } \\
\text { Curvature, Coarse }\end{array}$ & scale & $-24.5^{a}$ & $-27.3^{a}$ & -5.9 & $-50.4^{a}$ \\
\hline Elevation & $\mathrm{m}$ & $207.6^{\mathbf{a}, \mathbf{b}, \mathbf{c}, \mathbf{d}}$ & $196.7^{\mathbf{a}, \mathbf{b}}$ & $238.6^{d}$ & $174.8^{\mathrm{a}}$ \\
\hline $\begin{array}{l}\text { Distance to Karst } \\
\text { Landscape }\end{array}$ & $\mathrm{km}$ & $25.02^{\mathbf{a}, \mathbf{c}}$ & $52.47^{\mathrm{a}}$ & $11.09^{\mathrm{a}}$ & $1.51^{\mathrm{a}}$ \\
\hline Slope, Fine & percent rise & $3.2^{\mathrm{a}}$ & $3.3^{\mathrm{a}}$ & $2.9^{\mathbf{a}, \mathrm{b}}$ & $3.7^{\mathrm{a}}$ \\
\hline Slope, Medium & percent rise & $1.7^{\mathrm{a}, \mathrm{d}}$ & $1.9^{\mathrm{a}}$ & 1.1 & 2.1 \\
\hline Slope, Coarse & percent rise & 0.2 & $0.1^{\mathbf{d}}$ & $0.2^{\mathrm{a}}$ & 0.1 \\
\hline Viewshed & $\mathrm{km}^{2}$ & $499.6^{a}$ & 133.5 & $1090.2^{d}$ & $144.7^{\mathrm{a}}$ \\
\hline
\end{tabular}




\begin{tabular}{|c|c|c|c|c|c|}
\hline Variable & $\begin{array}{l}\text { Value } \\
\text { Represents }\end{array}$ & All Provinces & $\begin{array}{l}\text { Appalachian } \\
\text { Plateaus }\end{array}$ & Central Lowland & $\begin{array}{l}\text { Interior Low } \\
\text { Plateaus }\end{array}$ \\
\hline Aspect, East & $\begin{array}{l}\text { proportion of } \\
\text { Aspect }\end{array}$ & $10.00 \%$ & $10.26 \%$ & $5.26 \%$ & $17.39 \%$ \\
\hline Aspect, Flat & $\begin{array}{l}\text { proportion of } \\
\text { Aspect }\end{array}$ & $34.00 \%{ }^{\mathrm{a}}$ & $35.90 \%{ }^{a}$ & $34.21 \%^{\mathrm{a}}$ & $30.43 \%{ }^{a}$ \\
\hline Aspect, North & $\begin{array}{l}\text { proportion of } \\
\text { Aspect }\end{array}$ & $8.00 \%$ & $7.69 \%$ & $7.89 \%$ & $8.70 \%$ \\
\hline Aspect, Northeast & $\begin{array}{l}\text { proportion of } \\
\text { Aspect }\end{array}$ & $4.00 \%$ & $5.13 \%$ & $5.26 \%$ & $0.00 \%^{\mathrm{a}}$ \\
\hline Aspect, Northwest & $\begin{array}{l}\text { proportion of } \\
\text { Aspect }\end{array}$ & $10.00 \%$ & $5.13 \%$ & $15.79 \%$ & $8.70 \%$ \\
\hline Aspect, South & $\begin{array}{l}\text { proportion of } \\
\text { Aspect }\end{array}$ & $5.00 \%{ }^{\mathrm{a}}$ & $7.69 \%$ & $5.26 \%$ & $0.00 \%^{\mathbf{b}}$ \\
\hline Aspect, Southeast & $\begin{array}{l}\text { proportion of } \\
\text { Aspect }\end{array}$ & $8.00 \%$ & $7.69 \%$ & $10.53 \%$ & $4.35 \%$ \\
\hline Aspect, Southwest & $\begin{array}{l}\text { proportion of } \\
\text { Aspect }\end{array}$ & $11.00 \%$ & $10.26 \%$ & $10.53 \%$ & $13.04 \%$ \\
\hline Aspect, West & $\begin{array}{l}\text { proportion of } \\
\text { Aspect }\end{array}$ & $10.00 \%$ & $10.26 \%$ & $5.26 \%$ & $17.39 \%^{\mathbf{b}}$ \\
\hline TPI, Fine, flat & proportion of TPI & $33.00 \%^{\mathrm{a}}$ & $35.90 \%{ }^{\mathrm{a}}$ & $31.58 \%$ a, b & $30.43 \%^{\mathrm{a}}$ \\
\hline $\begin{array}{l}\text { TPI, Fine, } \\
\text { midslope }\end{array}$ & proportion of TPI & $4.00 \%$ & $2.56 \%$ & $5.26 \%$ & $4.35 \%$ \\
\hline TPI, Fine, ridge & proportion of TPI & $19.00 \%^{\mathrm{a}}$ & $17.95 \%$ & $15.79 \%$ & $26.09 \%$ \\
\hline $\begin{array}{l}\text { TPI, Fine, toe } \\
\text { slope }\end{array}$ & proportion of TPI & $14.00 \%^{\mathrm{a}}$ & $12.82 \%$ & $18.42 \%^{\mathrm{a}}$ & $8.70 \%$ \\
\hline $\begin{array}{l}\text { TPI, Fine, upper } \\
\text { slope }\end{array}$ & proportion of TPI & $13.00 \%$ & $10.26 \%$ & $13.16 \%$ & $17.39 \%$ \\
\hline TPI, Fine, valley & proportion of TPI & $17.00 \%^{\mathrm{a}}$ & $20.51 \%$ & $15.79 \%^{\mathrm{a}}$ & $13.04 \%^{\mathrm{a}}$ \\
\hline TPI, Medium, flat & proportion of TPI & $12.00 \%^{\mathrm{a}}$ & $17.95 \% \%^{\mathbf{a}, \mathbf{b}, \mathbf{c}}$ & $7.89 \%$ & $8.70 \%$ \\
\hline $\begin{array}{l}\text { TPI, Medium, } \\
\text { midslope }\end{array}$ & proportion of TPI & $0.00 \%$ & $0.00 \%$ & $0.00 \%$ & $0.00 \%$ \\
\hline $\begin{array}{l}\text { TPI, Medium, } \\
\text { ridge }\end{array}$ & proportion of TPI & $26.00 \%{ }^{a}$ & $17.95 \% \%^{\mathbf{a}, \mathbf{b}, \mathbf{c}}$ & $31.58 \%$ & $30.43 \%$ \\
\hline $\begin{array}{l}\text { TPI, Medium, toe } \\
\text { slope }\end{array}$ & proportion of TPI & $2.00 \%$ & $2.56 \%$ & $0.00 \%$ & $4.35 \%$ \\
\hline $\begin{array}{l}\text { TPI, Medium, } \\
\text { upper slope }\end{array}$ & proportion of TPI & $7.00 \%^{\mathrm{a}}$ & $10.26 \%{ }^{a}$ & $7.89 \%$ & $0.00 \%$ \\
\hline $\begin{array}{l}\text { TPI, Medium, } \\
\text { valley }\end{array}$ & proportion of TPI & $53.00 \%$ & $51.28 \%$ & $52.63 \%$ & $56.52 \%$ \\
\hline TPI, Coarse, flat & proportion of TPI & $2.00 \%$ & $0.00 \%$ & $5.26 \%{ }^{\mathrm{a}}$ & $0.00 \%$ \\
\hline $\begin{array}{l}\text { TPI, Coarse, } \\
\text { midslope }\end{array}$ & proportion of TPI & $1.00 \%$ & $0.00 \%$ & $2.63 \%$ & $0.00 \%$ \\
\hline TPI, Coarse, ridge & proportion of TPI & $10.00 \%^{\mathbf{a}, \mathbf{b}, \mathbf{d}}$ & $5.13 \%^{\mathrm{a}}$ & $15.79 \%^{\mathrm{a}}$ & $8.70 \%^{a}$ \\
\hline $\begin{array}{l}\text { TPI, Coarse, toe } \\
\text { slope }\end{array}$ & proportion of TPI & $1.00 \%$ & $0.00 \%$ & $2.63 \%$ & $0.00 \%$ \\
\hline $\begin{array}{l}\text { TPI, Coarse, } \\
\text { upper slope }\end{array}$ & proportion of TPI & $1.00 \%$ & $0.00 \%$ & $2.63 \%$ & $0.00 \%$ \\
\hline $\begin{array}{l}\text { TPI, Coarse, } \\
\text { valley }\end{array}$ & proportion of TPI & $85.00 \% \mathrm{a}, \mathrm{d}$ & $94.87 \%^{\mathrm{a}}$ & $71.05 \%^{\mathrm{a}}$ & $91.30 \%{ }^{\mathrm{a}}$ \\
\hline $\begin{array}{l}\text { Soil Order, } \\
\text { Alfisols }\end{array}$ & $\begin{array}{l}\text { proportion of Soil } \\
\text { Order }\end{array}$ & $73.74 \% \%^{\text {a, b, c }}$ & $79.49 \%{ }^{\text {a, c }}$ & $71.05 \%$ & $68.18 \%^{\mathrm{a}}$ \\
\hline $\begin{array}{l}\text { Soil Order, } \\
\text { Entisols }\end{array}$ & $\begin{array}{l}\text { proportion of Soil } \\
\text { Order }\end{array}$ & $3.03 \%$ & $5.13 \%$ & $2.63 \%$ & $0.00 \%$ \\
\hline
\end{tabular}




\begin{tabular}{|l|l|l|l|l|l|}
\hline Variable & $\begin{array}{l}\text { Value } \\
\text { Represents }\end{array}$ & All Provinces & $\begin{array}{l}\text { Appalachian } \\
\text { Plateaus }\end{array}$ & Central Lowland & $\begin{array}{l}\text { Interior Low } \\
\text { Plateaus }\end{array}$ \\
\hline $\begin{array}{l}\text { Soil Order, } \\
\text { Inceptisols }\end{array}$ & $\begin{array}{l}\text { proportion of Soil } \\
\text { Order }\end{array}$ & $8.08 \%$ & $7.69 \%$ & $7.89 \%$ & $9.09 \%^{\circ}$ \\
\hline $\begin{array}{l}\text { Soil Order, } \\
\text { Mollisols }\end{array}$ & $\begin{array}{l}\text { proportion of Soil } \\
\text { Order }\end{array}$ & $11.1 \%^{\mathbf{a}}$ & $0.00 \%$ & $15.79 \%^{\mathbf{a}}$ & $22.73 \%^{\mathbf{a}}$ \\
\hline $\begin{array}{l}\text { Soil Order, } \\
\text { Ultisols }\end{array}$ & $\begin{array}{l}\text { proportion of Soil } \\
\text { Order }\end{array}$ & $4.04 \%^{\mathbf{a}}$ & $7.69 \%$ & $2.63 \%^{\mathbf{a}}$ & $0.00 \%^{\mathbf{a}}$ \\
\hline $\begin{array}{l}\text { Province, } \\
\text { Appalachian } \\
\text { Plateaus }\end{array}$ & $\begin{array}{l}\text { proportion of } \\
\text { Province }\end{array}$ & $39.0 \%^{\mathbf{a}, \mathbf{d}}$ & & & \\
\hline $\begin{array}{l}\text { Province, Central } \\
\text { Lowland }\end{array}$ & $\begin{array}{l}\text { proportion of } \\
\text { Province }\end{array}$ & $38.00 \%^{\mathbf{a}, \mathbf{d}}$ & & \\
\hline $\begin{array}{l}\text { Province, Interior } \\
\text { Low Plateaus }\end{array}$ & $\begin{array}{l}\text { proportion of } \\
\text { Province }\end{array}$ & $23.00 \%^{\mathbf{a}, \mathbf{b}, \mathbf{c}, \mathbf{d}}$ & & & \\
$\mathbf{a}=p<.05$, Paleoindian versus Random Point \\
$\mathbf{b}=p<.05$, Paleoindian versus Early Archaic \\
$\mathbf{c}=p<.05$, Paleoindian versus Late Archaic \\
$\mathbf{d}=p<.05$, Early Archaic versus Late Archaic
\end{tabular}

\subsubsection{Physiographic Province and TRI}

Paleoindian site distributions indicate a significant under-representation in the Appalachian Plateaus and over-representation in the Central Lowlands and Interior Low Plateaus. These proportions are interpreted as evidence for differential use of the study area by Paleoindian groups with some avoidance of the Appalachian Plateaus. Based on values derived from fineresolution TRI calculations at random point locations, the Interior Low Plateaus $(\bar{x}=132.5$, slightly rugged), not the more commonly assumed Appalachian Plateaus $(\bar{x}=106.2$, nearly level), possess the most rugged terrain in southern Ohio. The glaciated Central Lowlands terrain $(\bar{x}=99.7$, nearly level) is the least rugged. The ruggedness of the Interior Low Plateaus is primarily related to the resistant Berea Sandstone-capped Allegheny Escarpment, which produces high relief and represents the Appalachian Plateaus eastern boundary (Brockman, 2006). Although avoidance of the Appalachian Plateaus is indicated, the rugged Interior Low Plateaus has a robust Paleoindian assemblage suggesting that avoidance behavior was not applied at the physiographic scale but instead was more situationally dictated. The high interperiod variability in site distributions between provinces (Table 2-3) may indicate transitioning settlement-subsistence strategies through time for groups within the Interior Low Plateaus.

\subsubsection{Distance to Large and Large-Medium River Confluence Pt.}

Distance to river confluences, both for large and large-medium rivers show some patterning (see also Figure S1 in Supplemental Information). In the comprehensive model, Paleoindian sites are located at a significantly closer distances to both large river and large-medium river confluences than random supporting the notion that major drainage confluences attracted Paleoindians for aggregation events, travel, or to hunt concentrated game. By individual province, Paleoindian sites are significantly closer than random to large river confluences in the Appalachian Plateaus and the Interior Low Plateaus, but not in the Central Lowlands. Only in the Central Lowlands were Paleoindian sites significantly closer to medium-large river confluences than random. 


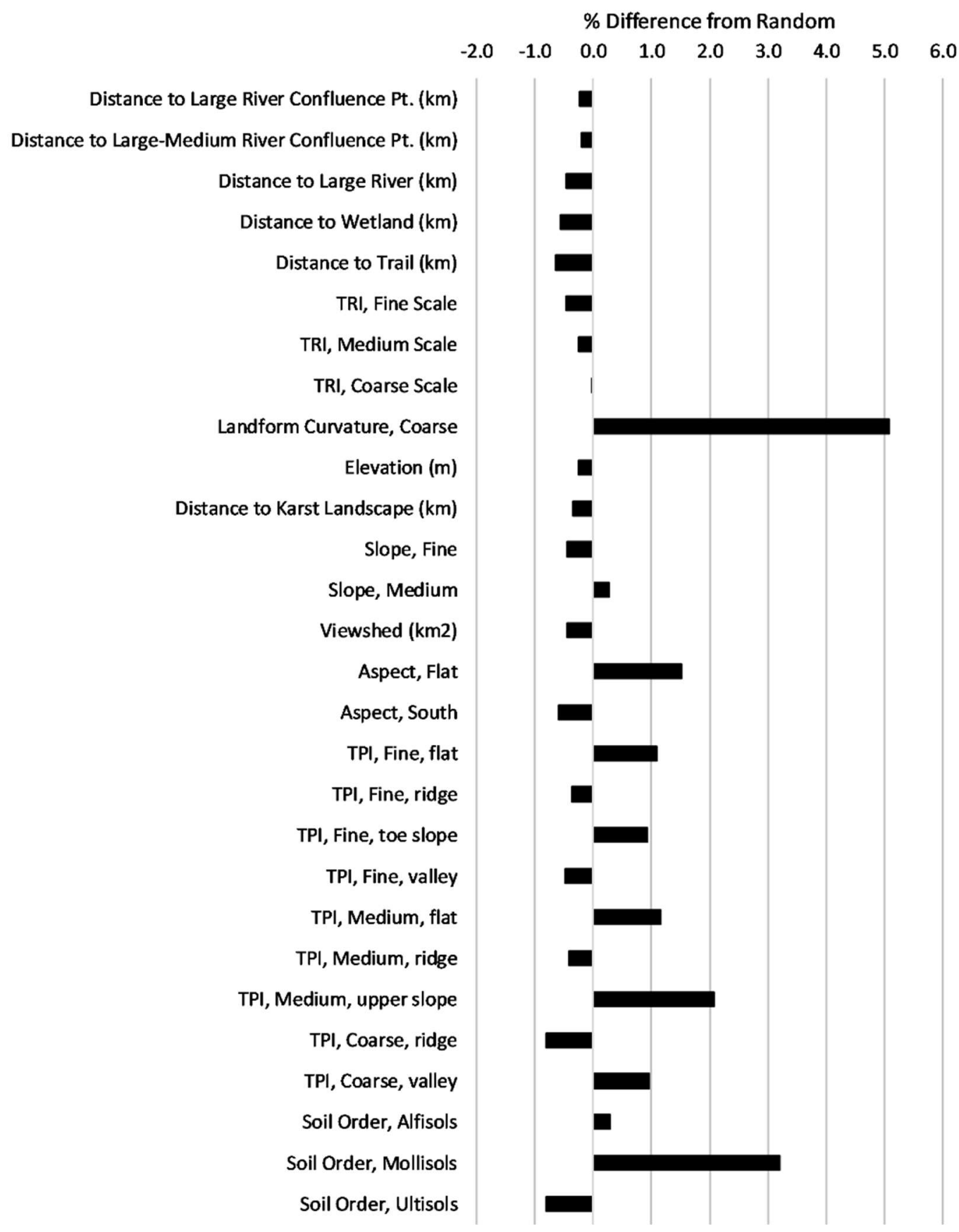

Figure 34. Percent-change graph by variable type of Paleoindian site values from the assumption of randomness designated as ' 0 ' on horizontal scale (x100). Only statistically significant variables from Table 2-3 are included.

\subsubsection{Distance to Medium and Large Rivers}

Only large rivers show statistically significant patterning in relation to Paleoindian and Archaic site proximities (Figure S1 in Supplemental Information). Paleoindian sites are significantly closer to large rivers than random points or Archaic sites in all three provinces. This finding 
supports previous researchers who hypothesized intensive use of large river drainages by Paleoindians, especially during initial colonization (see Table 2-1).

\subsubsection{Distance to Wetlands}

In all provinces, Paleoindian sites are significantly closer than random to wetlands, perhaps highlighting a pattern of exploitation of unique plant-animal communities. Paleoindian sites also tend to be closer to wetlands than either Early Archaic or Late Archaic sites, although not all coassociations are significant (see Tables S2-S5 in Supplemental Information).

\subsubsection{Distance to Trail}

Paleoindian sites are significantly closer than random to potential trail systems in all provinces (see Figure S2 in Supplemental Information). This finding suggests the importance of these early trail systems for Paleoindians. Except for the Central Lowlands, Paleoindian sites are located slightly closer to potential trails than Archaic sites in the more rugged terrain of the Appalachian Plateaus and Interior Low Plateaus.

\subsubsection{Distance to Sand Dune}

In the comprehensive model, Paleoindian sites reveal no significant patterning with distance from sand dunes. By individual province, however, Paleoindian sites are significantly more distant to dunes in the Interior Low Plateaus and Central Lowlands but closer in the Appalachian Plateaus. Although a lack of correlation in the current dataset contrasts with previous researchers (see Table 2-1), Sandy Springs (33AD30) (Cunningham, 1973; Purtill, 2017, 2017; Purtill \& Kite, 2015; Seeman et al., 1994) is one possible caveat to this finding. Sandy Springs is a large, multiple occupation Paleoindian site associated with a large dunefield on an Ohio River Pleistocene terrace (Purtill, 2017a; Seeman et al., 1994). Since Sandy Springs only represents a single site in the modeling process, the true importance of this sandy ecosystem for Paleoindian occupation may be under-represented in this study. Paleoindian and Archaic sites also show no significant difference in distance to sand dunes suggesting little chronological variation in use of sandy ecosystems through time.

\subsubsection{Landform Curvature and Slope}

Random points for both Landform Curvature and Slope variables show significant variation from Paleoindian sites at several resolutions and provinces (see Figures S4-S5 in Supplemental Information). Paleoindian sites tend to be located on more level landforms than random points regardless of resolution or province. At both the fine- and coarse-resolution comprehensive scale, Paleoindian sites are situated on landforms more convex than random. In the Interior Low Plateaus, the reverse is true as Paleoindian sites are on increasingly concave landforms. Some between-period diversity also was documented as Paleoindian sites are located on significantly more convex landforms than Early Archaic sites in the Central Lowlands at fine resolution. 


\subsubsection{Distance to Karst Landscape}

Paleoindian sites are closer than random to karst landscapes in all provinces except the Appalachian Plateaus where they are more distant (see Figure S3 in Supplemental Information). Proximity to karst is especially low for Paleoindian sites $(\bar{x}=1.51 \mathrm{~km})$ in the Interior Low Plateaus when compared to random point counterparts $(\bar{x}=63.43)$. In southern Ohio, much of the karst landscape occurs within the Interior Low Plateaus province known for supporting prairie and savanna ecoregions (Fenneman and Johnson, 1946), especially the Outer Bluegrass Region (see Table 2-1). This may indicate a preference for more open prairie biomes.

\subsubsection{Elevation and Viewshed}

Study wide, Paleoindian sites are located at lower elevations with more restricted viewsheds than random. This trend also generally holds between Paleoindian versus Archaic site locations although with some exceptions. In the Appalachian Plateaus, Paleoindian sites are located at significantly lower elevations than Early Archaic sites, but do not deviate statistically from Late Archaic site locations. In general, these results do not support the oft repeated idea that Paleoindians purposely occupied elevated topographic positions that afforded broad overlooks to monitor game or set ambushes (see Table 2-1).

\subsubsection{Aspect}

The primary pattern observed for aspect direction is the preference for level, aspect-less landforms regardless of province. At the comprehensive level, Paleoindian sites in comparison to random are significantly under-represented on south- to southwest-facing landforms. This result contrasts with some (see Table 2-1) who suggested a preference for south- and southwest-facing exposures in the Appalachian Plateaus to maximize insolation. Interestingly, a preference for south-facing landforms was identified for Early Archaic sites, but only in the Interior Low Plateaus. In the Interior Low Plateaus, Paleoindian sites also significantly deviate from random in being under-represented on northeast-facing aspects.

\subsubsection{TPI}

Paleoindian sites tend to be located on flat topographic positions defined at the fine resolution for all physiographic provinces. At the medium and coarse resolutions, associations between Paleoindian sites and flat topographic positions are mixed. There is a significant underrepresentation of Paleoindian sites on ridge topographic positions, regardless of variable resolution, a trend which fails to support the hypothesis that Paleoindians sought higher landforms with broad overlooks (see Table 2-1). Paleoindian sites are under-represented on ridges at all resolutions and models, especially when compared to Early Archaic sites which may suggest increased exploitation of higher elevations by Early Archaic populations (see section 4.2.9 for supporting argument regarding elevation variable). 


\subsubsection{Soil Order}

When compared to random points for the entire study area, Paleoindian sites are significantly over-represented on Mollisols and Alfisols and under-represented on Ultisols. The preference for Mollisols versus other soil orders by Paleoindians is especially strong in the Interior Low Plateaus and Central Lowlands but is not significant in the Appalachian Plateaus. In the Interior Low Plateaus, Mollisols are over-represented for Paleoindian (23\%), Early Archaic (11\%), and Late Archaic (17\%) sites when compared to random points which reflect $<1 \%$ of the province. This may reflect a preference by foragers in general for prairie environments in the upper Ohio Valley well into the Holocene.

\subsubsection{Binary Logistic Regression}

A binary logistic regression was calculated with the 31 independent variables identified as significant between Paleoindian site and random point locations for the comprehensive model (Table 2-3, Figure 2-4). Several tests were run using different entry methods but ultimately it was determined that the 'enter' method produced the best fitted model. Final results were modestly successful with a -2LL value of 301.802 and a Nagelkerke $\mathrm{R}^{2}$ value of 0.593 suggesting that this variable combination accounts for $\sim 59 \%$ of Paleoindian dataset variance. Four independent variables contained both a significant Wald statistic $(p<0.05)$ and elevated OR value ( $>3$ ): Province, Interior Low Plateaus; Soil Order, Mollisol; Province, Central Lowland; Soil Order, Alfisols. This suggests that these four variables were positively influential in factors related to site placement during the Paleoindian period.

\section{Discussion}

Numerous substantive theoretical implications concerning eastern U.S. Paleoindian land-use behavior have been assessed as a result of this study. In general, this study finds empirical support for several hypothesized co-associations between background environmental data and Paleoindian sites (Table 2-4). Evidence fails to support the concept of a single, unified Paleoindian settlement-subsistence pattern in southern Ohio but instead suggests variability in Paleoindian land-use behavior between individual physiographic provinces. Since current reconstructions of past climate, vegetation, and precipitation patterns (see Section 2.0) illustrate a dynamic, quickly evolving environment during the late Pleistocene for southern Ohio, this finding generally supports previous assertions of regional economic diversity for Paleoindian lifeways, especially between glaciated and unglaciated landscapes (Anderson \& Gillam, 2000; Lepper, 1988; Loebel, 2012; Meltzer \& Smith, 1986; Roper \& Lepper, 1991; Smallwood, 2012; Witthoft, 1954). An example of the type of late Pleistocene Ohio environmental and resource diversity potentially encountered by Paleoindians was summarized nicely by palynologist Linda Shane:

"If human populations preferred warmer conditions, then the Till Plains areas [Central Lowlands] would have been chosen. If they preferred diversity, both spruce and deciduous forests were present on the Plateau, whereas the spruce forest was almost gone on the Till Plains. On the other hand, edge areas may have 
increased in the more open forests of the Till Plains, which in turn might have supported increased faunal diversity (Shane, 1994, p. 12)."

Environmental reconstructions such as Shane's suggest that southern Ohio contained a diverse array of habitats and resource bases during the late Pleistocene. Given the scale of mobility posited for Paleoindians it seems reasonable to conclude that individual bands would have traveled through and exploited the entire upper Ohio Valley landscape and not just specific provinces or environmental settings. If accurate, this suggests that efficient land use would have required multiple extraction strategies, perhaps ones employed on a seasonal basis as suggested by Lepper (1988) for the Appalachian Plateaus.

Table 2-4. Summary of Paleoindian results and comparison with Early Archaic patterns

\begin{tabular}{|c|c|c|c|}
\hline $\begin{array}{l}\text { Proposed Environment } \\
\text { Co-association with } \\
\text { Paleoindian Sites }\end{array}$ & $\begin{array}{l}\text { Archaeological } \\
\text { Support } \\
\text { (Paleoindian vs. } \\
\text { random point) } \\
\end{array}$ & $\begin{array}{l}\text { Early Archaic } \\
\text { pattern deviates } \\
\text { from Paleoindian } \\
\text { pattern }\end{array}$ & Explanation \\
\hline karst landscape & Yes & No & $\begin{array}{l}\text { Paleoindian sites tend to be located closer to karst } \\
\text { landscapes than random, especially in Interior Low } \\
\text { Plateaus. }\end{array}$ \\
\hline major river drainage & Yes & Yes & $\begin{array}{l}\text { Paleoindian sites tend to be located closer to large } \\
\text { rivers ( }>2590 \mathrm{~km}^{2} \text { basin area) than random, but the } \\
\text { same trend is not present for medium rivers. At the } \\
\text { comprehensive scale, Paleoindian sites are } \\
\text { significantly closer than Early Archaic sites to large } \\
\text { rivers. }\end{array}$ \\
\hline $\begin{array}{l}\text { major drainage } \\
\text { confluence areas }\end{array}$ & Yes & No & $\begin{array}{l}\text { Paleoindian sites tend to be located closer to large and } \\
\text { large-medium river confluences than random, } \\
\text { although results are mixed based on physiographic } \\
\text { province. Increased proximity to large river } \\
\text { confluences was found in Appalachian Plateaus and } \\
\text { Interior Low Plateaus. }\end{array}$ \\
\hline wetlands & Yes & No & $\begin{array}{l}\text { Paleoindian sites tend to be located closer to wetlands } \\
\text { than random in all provinces. }\end{array}$ \\
\hline $\begin{array}{l}\text { overlooks or high } \\
\text { elevations }\end{array}$ & No & Yes & $\begin{array}{l}\text { Paleoindian sites tend to be at lower elevations with } \\
\text { reduced viewsheds and an avoidance of ridge } \\
\text { landforms compared to random. Early Archaic sites } \\
\text { tend to be at higher elevations, with broader } \\
\text { viewsheds, and more commonly on ridge landforms } \\
\text { than Paleoindian sites. }\end{array}$ \\
\hline $\begin{array}{l}\text { sandy environments, } \\
\text { especially dunefields }\end{array}$ & Partially & No & $\begin{array}{l}\text { Paleoindian sites are only located significantly closer } \\
\text { than random to sand dunes in the Appalachian } \\
\text { Plateaus. }\end{array}$ \\
\hline $\begin{array}{l}\text { animal trails, migration } \\
\text { paths, river corridors } \\
\text { used for transportation }\end{array}$ & Yes & Partially & $\begin{array}{l}\text { Paleoindian sites tend to be located closer than } \\
\text { random to potential trail systems. In Central } \\
\text { Lowlands, Early Archaic sites are significantly closer } \\
\text { to potential trails than Paleoindian sites. }\end{array}$ \\
\hline $\begin{array}{l}\text { Avoidance of rugged } \\
\text { terrain or variable use of } \\
\text { physiographic regions }\end{array}$ & Yes & Partially & $\begin{array}{l}\text { Paleoindian sites tend to be located on more level } \\
\text { landforms than random, regardless of province. In the } \\
\text { Interior Low Plateaus, Early Archaic sites are under- } \\
\text { represented whereas Paleoindian sites are over- } \\
\text { represented in this province. In the Appalachian } \\
\text { Plateaus, Early Archaic sites are located on } \\
\text { significantly rougher terrain than Paleoindian sites. }\end{array}$ \\
\hline Southern exposures & No & Partially & $\begin{array}{l}\text { Paleoindian sites demonstrate no pattern of south- } \\
\text { facing aspects in any province. In the Interior Low } \\
\text { Plateaus, Paleoindian sites are under-represented in }\end{array}$ \\
\hline
\end{tabular}




\begin{tabular}{|l|l|l|l|}
\hline $\begin{array}{l}\text { Proposed Environment } \\
\text { Co-association with } \\
\text { Paleoindian Sites }\end{array}$ & $\begin{array}{l}\text { Archaeological } \\
\text { Support } \\
\text { (Paleoindian vs. } \\
\text { random point) }\end{array}$ & $\begin{array}{l}\text { Early Archaic } \\
\text { pattern deviates } \\
\text { from Paleoindian } \\
\text { pattern }\end{array}$ & Explanation \\
\hline & & & $\begin{array}{l}\text { northeast-facing aspects. Early Archaic sites only } \\
\text { deviate from the Paleoindian pattern in the Interior } \\
\text { Low Plateaus, where there is an over-representation } \\
\text { of south-facing aspects. }\end{array}$ \\
\hline $\begin{array}{l}\text { Eastern U.S. tall-grass } \\
\text { prairie }\end{array}$ & Yes & No & $\begin{array}{l}\text { Paleoindian sites are over-represented on Mollisols } \\
\text { and in the Interior Low Plateaus. }\end{array}$ \\
\hline
\end{tabular}

The Interior Low Plateaus province is highly variable regarding Paleoindian and later Archaic land-use behavior. This study demonstrates that Paleoindian sites are statistically overrepresented in the Interior Low Plateaus, especially landforms characterized by Mollisols and karst topography. The importance of the Interior Low Plateaus province also was demonstrated in the binary logistic regression model where the odds of a Paleoindian site being situated increases $\sim 14 \mathrm{x}(\mathrm{OR}=13.989)$ compared to other provinces. This pattern appears similar to Paleoindian settlement patterns in the Tennessee and Cumberland Valleys (Broster et al., 2013, p. 309; Freeman et al., 1996; Kenneth B. Tankersley, 1996) and may represent a unified Midsouth settlement pattern of exploitation of a mixed woodland and open prairie environments as suggested for the general Southeast region (T. M. N. Lewis, 1953) and perhaps into northern Mexico (Johnson et al., 2006). Pollen reconstructions of late Pleistocene environments in the Interior Low Plateaus indicate open, grassy, savannah-like environments on broad alluvial plains of the Ohio River (Purtill, 2012, pp. 42-43, 2017). Such an environment, in addition to the surrounding wooded dissected uplands, would have supported a diverse array of animal and plant species including megafauna and herd animals, but also smaller woodland species. Hollenbach (2009) has suggested a foraging subsistence pattern for the Middle Tennessee River focused on small mammals, birds, fishes, reptiles, invertebrates, and wild plants (Broster et al., 2013, p. 309).

Previous research of spatial distribution of diagnostic bifaces lends further credence to the idea of strengthening cultural ties between Paleoindian groups throughout the Interior Low Plateaus province. This interaction is evidence by the distribution of Cumberland bifaces, which are hypothesized to post-date Clovis and represent 'Middle Paleoindian' (12.8-12.6 cal yrs B.P.) and are extremely common in the Tennessee and Cumberland river valleys (D. S. Miller et al., 2013, p. 10; Tune, 2016), appear to have their northern-most boundary in southern Ohio (Prufer \& Baby, 1963; Seeman \& Prufer, 1982; Stothers, 1996, p. 181; Tune, 2016, p. 311). Cumberland bifaces were especially common at Sandy Springs, where Seeman et al. (1994, pp. 82, 88) documented a transition away from use of Upper Mercer chert from Ohio for earlier Clovis bifaces to exploitation of southern toolstone sources, potentially including Ft. Payne chert from Tennessee, for later Paleoindian biface styles. Seeman et al. (1994, p. 83) found evidence for "....increased utilization of Sandy Springs by bands operating to the southeast in central Kentucky and Tennessee". This interpretation also corresponds with Anderson's findings which illustrates his Middle Paleoindian Regional Tradition as including the Tennessee, Cumberland, and Ohio valleys (Anderson, 1990, p. 192, 1996, p. 36). By Early Archaic times, the distribution of sites is significantly under-represented in the Interior Low Plateaus suggesting disruption from this earlier settlement pattern and perhaps reflecting decreased interaction with Midsouth populations and increased regionalization. 
This study also does not support the notion that Paleoindians favored high topographic positions with expansive viewsheds from which megafauna or migrating herds could be monitored. This original view closely aligned with the belief Paleoindians as big-game specialist (Kelly \& Todd, 1988) leading Tankersley (1990, p. 281) in an earlier synthesis to suggest that "all of the Early Paleoindian sites sampled can be explained in terms of the procurement and/or processing of either game or lithic raw material". Since recent research has downplayed the exclusivity of hunting in Paleoindian subsistence economies (Gingerich, 2011; Hollenbach, 2009; McWeeney \& McWeeney J., 2013), at least for deciduous woodland environments, the importance of overlook positions may have been overstated in previous literature. It is interesting to note that no significant difference in viewshed area was documented between Paleoindian $(\bar{x}=499.6$ $\left.\mathrm{km}^{2}\right)$, Early Archaic $\left(\bar{x}=660.6 \mathrm{~km}^{2}\right)$, and Late Archaic $\left(\bar{x}=480.7 \mathrm{~km}^{2}\right)$ sites in any of the three physiographic provinces tested (see Table 2-3 and Tables S2-S5 in Supplemental Information). This similarity suggests little variation in the use of landforms by Paleoindian and both Archaic groups, at least with regards to visibility of the surrounding landscape.

Study results support the idea that Paleoindians used major river drainages, trail complexes, and river confluences for movement throughout southern Ohio, a pattern that likely extends to the entire eastern U.S. One notable exception to this trend is the absence of Paleoindian sites in the lower Scioto Valley (Figure 4 and 2-6; see also Figures S1-S5 in Supplemental Information). A similar deficiency was not observed for Archaic sites (see Figure 2-2) thus mitigating, at least to a degree, the potential that collector bias accounts for the absence of lower Scioto Valley Paleoindian sites. 


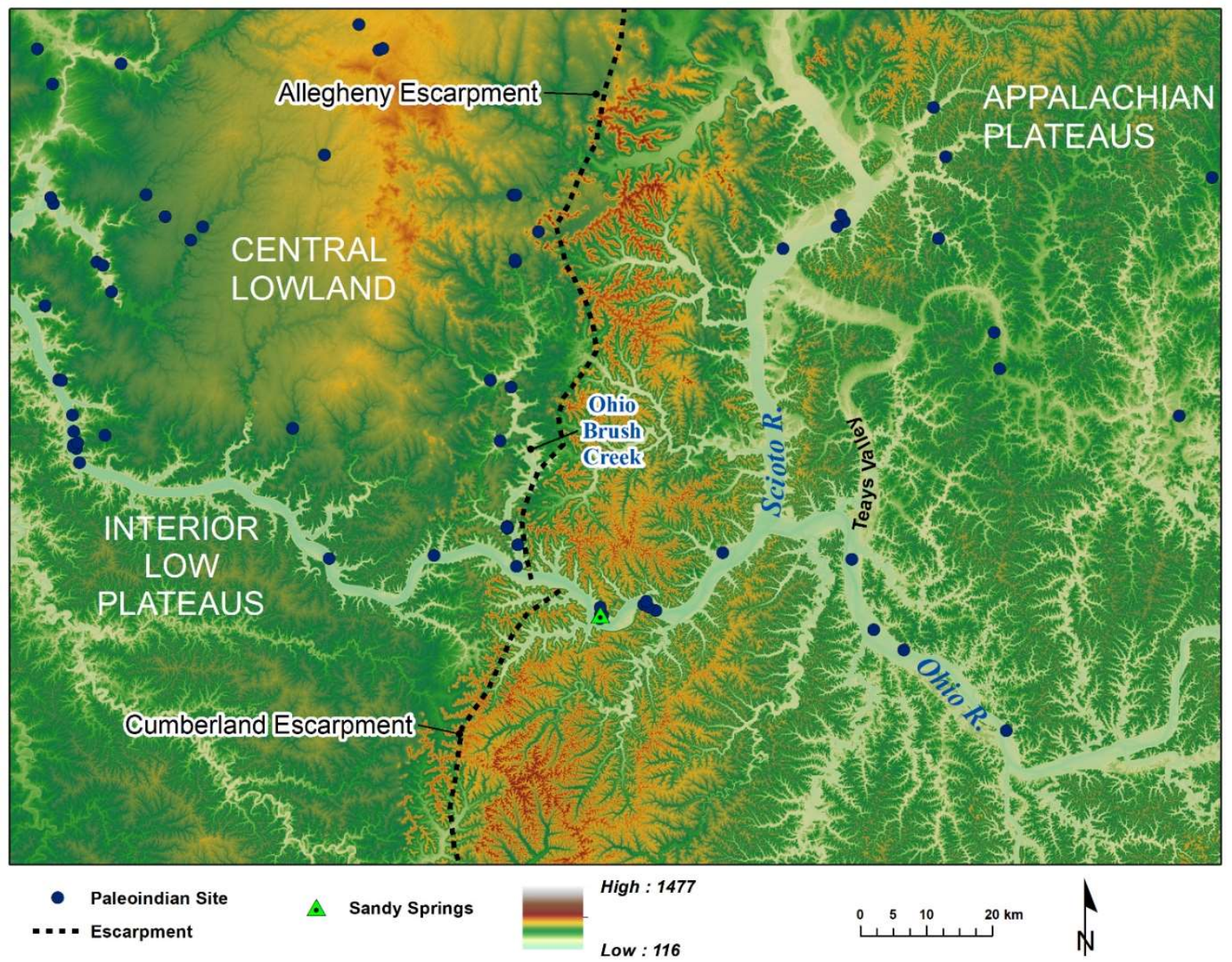

Figure 4. Enlarged view of a portion of Figure 2-1 showing Interior Low Plateaus and location of Paleoindian sites in reference to the lower Scioto River and Ohio Brush Creek valleys. Allegheny-Cumberland escarpment complex also illustrated. Elevations presented in meters above mean sea level. 


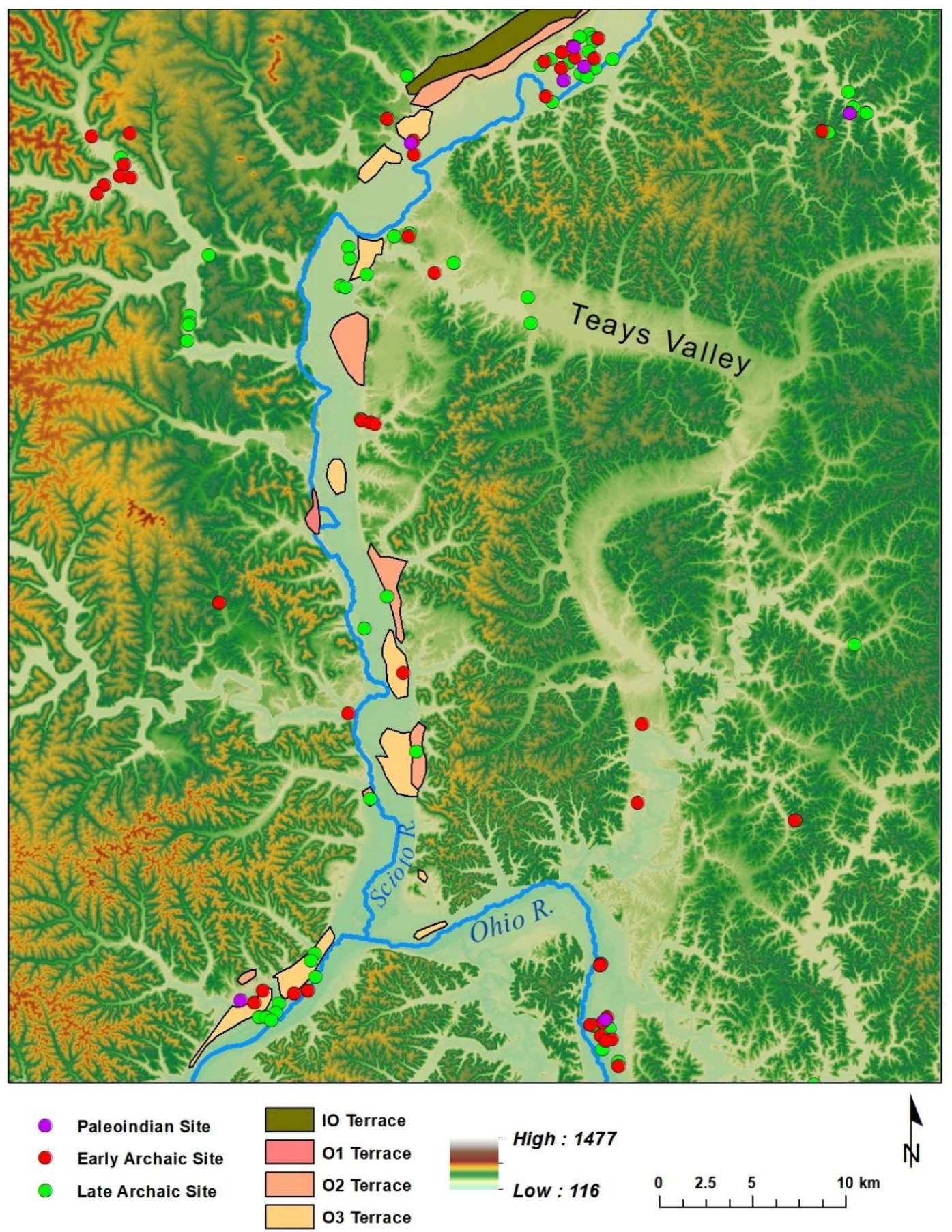

Figure 56. Enlarged view of lower Scioto River Valley, primary tributaries, alluvial terraces, and distribution of archaeological sites. Terraces mapped after Pavey et al., 1999 (IO = Illinoian Terrace; O1 = 22-18 ka RCYBP; O2 = 18-15 ka RCYBP; O3 = 15-14 ka RCYBP).

The lower Scioto River reach is an active meandering system with abundant meander scars, meander scroll bars, oxbow lakes, and backswamps. Although Holocene geomorphic processes undoubtedly have eroded most of the pre-Holocene landforms that once existed on the valley bottomland, perhaps as much as $85 \%$, geological mapping of the lower Scioto suggests remnant 
late Pleistocene outwash terraces are present in the valley (Pavey et al., 1999; see Figure 2-6). At least some Early and Late Archaic sites are located on these pre-Holocene landforms, but Paleoindian sites are absent between the Scioto's junction with the Teays Paleovalley and its mouth at the Ohio River (see Figure 2-6). Paleoindian sites are present upriver of the Teays Paleovalley as noted by Prufer $(1975$, p. 315) when he stated that this reach is "rich in PaleoIndian remains...(n)early 50 per cent of the 66 fluted points known from Ross County were found in the Scioto Valley, usually on the high terraces" (see also the northern two-thirds of Figure 2-6). Figure 2-6 also illustrates that Paleoindian sites are present on high surfaces in the Ohio River Valley proper near the mouth of the Scioto River. Strikingly, Paleoindian sites also are absent from tributaries that immediately feed the lower Scioto River (see Figure 2-6). Although site absence in the Scioto bottomlands conceivably could be the result of erosion by a dynamic meandering river system, erosional processes in tributaries would have been less severe. If Paleoindians were traversing these tributaries, it is reasonable to expect sites to be in these drainages as is the case for the Early and Late Archaic periods.

It is proposed that the absence of sites in the lower Scioto Valley and immediate tributaries reflects a cultural pattern of deliberate avoidance by Paleoindians. The Scioto River was a major outwash channel for the Scioto sublobe of the Laurentide Ice Sheet (Fullerton, 1986; Glover et al., 2011; Kempton \& Goldthwait, 1959). Radiocarbon dating of till and outwash deposits north of the Wisconsin glacial margin suggest that significant pulses of icy meltwater and outwash were transported down the lower Scioto River as early as $\sim 23 \mathrm{ka}$, synchronous to the Last Glacial Maximum and subsequent lobe retreats and advancements (Frokling \& Szabo, 1998; Fullerton, 1986; Glover et al., 2011; Goldthwait, 1958). Following the termination of meltwater pulses, the Scioto River would have continued to transport substantial reworked glaciofluvial sediments during the early Holocene given that portions of the upper Ohio River appear to have been braided during this time (Rogers, 1990). During the late Pleistocene, eolian deflation of braid bars and alluvial plains likely was common and would have increased air dustiness and deposition of fine sediment in rivers potentially creating an inhospitable environment for human occupation or travel. Elevated dustiness is known to increase health issues related to respiratory functions (Karanasiou et al., 2012; Mitsakou et al., 2008). Excessive fine sediment in rivers may impair hyporheic flow and negatively impact certain fish and macroinvertebrate species (e.g., Ryan, 2010). Such conditions, especially if changes in flood regime and channel geomorphology occurred, may have dissuaded migrating herd animals from traveling through the lower Scioto River due to habitat degradation and fragmentation (e.g., Death et al., 2015). A similar interpretation is offered for the absence of Paleoindian sites, specifically Clovis, in the lower Mississippi River (Kidder et al., 2008, p. 1258; Rittenour et al., 2005).

About $40 \mathrm{~km}$ west of the lower Scioto River is the smaller Ohio Brush Creek valley which contains a robust 13\% of all southern Ohio Paleoindian sites (Figure 4; see also Figures S4-S5 in Supplemental Information). This relatively high site density in such a small drainage suggest intense utilization of this valley during the late Pleistocene. Ohio Brush Creek flows along the western edge of the Allegheny Escarpment and was not a major glacial outwash outlet, thus potentially mitigating many of the environmental threats hypothesized to be associated with the lower Scioto. This low-relief valley within an otherwise highly dissected, rugged, part of southern Ohio may have provided a natural north-south travel corridor for both human groups 
and migrating game moving between the unglaciated Ohio River area and glaciated Central Lowlands (see also Figures S4-S5).

Utilization of the Ohio Brush Creek Valley may also be part of a larger north-south trail system recently suggested by Purtill (2017a) for the Allegheny-Cumberland Escarpment that connects the glaciated upper Ohio Valley with the unglaciated Tennessee and Cumberland Valleys (see also Seeman \& Prufer, 1982, p. 157). This distinctive $\sim 800 \mathrm{~km}$ long escarpment complex marks the eastern edge of the Interior Low Plateaus and is a prominent, highly visible landscape that may have guided Paleoindians during seasonal logistical mobilizations (Boulanger \& Lyman, 2014; Ellis, 2011; Eren \& Redmond, 2011). Previous research has suggested that physiographic boundaries such as escarpments were important travel aids for the exploration of terra incognita (Kelly, 2003) and would have been some of the initial natural landmarks identified by early Paleoindians (Shane Miller, 2016; Tune, 2016, p. 311). Once established, such landmarks likely continued to serve as travel aids even after local Paleoindian populations began to learn their landscape.

Finally, although direct comparisons between Paleoindian and Early Archaic land-use behavior was not a primary goal of this study, results document several trends in southern Ohio worthy of mention. Despite the fact that long-distance seasonal rounds and a reliance on high-quality bedded toolstones helps easily distinguish Paleoindian and Early Archaic economies on one level (Anderson, 1995; Boulanger et al., 2015; Daniel Jr., Moore, \& Caynor, 2013; Ellis, 2011; Kelly \& Todd, 1988; Seeman, 1994; Kenneth B. Tankersley, 1990), the degree to which other aspects of land-use behavior correspond through time remains debated with many archaeologists suggesting subsistence-settlement continuity in the southern boreal-deciduous biomes of the unglaciated eastern U.S. (Bryan, 1977; Gardner, 1977; Lepper, 1988; Meltzer, 1988; Meltzer \& Smith, 1986, pp. 16-19; Pevny et al., 2018; see also Purtill, 2009, p. 591). In contrast, others argue that Paleoindian land-use was distinct from the later Archaic pattern primarily due to environmental change, biome transition, and increasing populations and social complexity (Koldehoff \& Loebel, 2009; Seeman \& Prufer, 1982, p. 163; Kenneth B. Tankersley, 1990, p. 281).

As summarized in Table 2-4 and in Supplemental Information, this study found no consistent pattern regarding the degree of continuity in land-use behavior between Paleoindian and Early Archaic groups in southern Ohio. Although most tested variables show similar modeled values for Paleoindian and Early Archaic sites, thus suggesting similarity in landform use, at least some dissimilarity emerged. Whereas $23 \%$ of the entire Paleoindian site database derives from the Interior Low Plateaus physiographic province, possibly reflecting extension of a Midsouth settlement pattern into southern Ohio, this province appears relatively depopulated during the succeeding Early Archaic period (8\%). Although the exact nature of this under-representation remains unknown, it is reasonable to interpret this trend as an adjustment or shift in land-use strategies for the province during the Paleoindian to Early Archaic transition. In the unglaciated Appalachian Plateaus, study results indicate an increased use of elevated landforms such as ridges and upper slopes by Early Archaic groups probably reflecting continued exploration of new lands, including areas largely avoided previously by Paleoindians. In the Central Lowlands, Paleoindian sites tend to be located on more convex, sloping landforms when compared to Early Archaic sites. Moreover, the Central Lowlands is the only provinces where Early Archaic sites, 
not Paleoindian sites, are situated closer to potential trail systems. The exact reason for this variation is not fully understood but again reinforces the basic study finding that Paleoindians engaged in distinct land-use behaviors as they encountered the heterogeneous southern Ohio landscape.

\section{Conclusions}

Many of our current perspectives on eastern U.S. Paleoindian lifeways are based on purported co-associations between site location and environmental or topographic setting. The magnitude and direction of these co-associations often has been based on qualitative observations and not empirical testing. Since many of these perspectives have been incorporated into theory building, assessing their validity is required if we wish to construct accurate models concerning the social, ideological, economic, and political condition of early humanity (Jordan, 2008). Through a combined GIS and statistical modeling approach, this study has empirically tested several hypothesized environmental co-associations and advanced new observations concerning late Pleistocene land-use behavior in the upper Ohio Valley.

Paleoindians did not practice a uniform land-use strategy in southern Ohio but instead adapted their strategies to fit local conditions. Especially noteworthy is land use in the Interior Low Plateaus province that potentially reflects interaction between Paleoindian populations in the Cumberland, Tennessee, and upper Ohio drainages, especially sometime after Clovis. From a methodological perspective, this study also demonstrates that the inclusion of Early and Late Archaic sites in the modeling process helps constrain negative effects of collector bias when interpreting Paleoindian site distributions. Since the potential impact of collector bias has been considered extensively in Paleoindian research, it important to realize that cross-temporal approaches as applied here may provide a means to mitigate the confounding influence of such bias on the archaeological record.

\section{Acknowledgements}

Portions of this research is supported by a 2016 NASA West Virginia Space Grant Consortium Graduate Research Fellowship. I also would like to thank the following individuals for their help with this research and the writing of this article: Jamison Conley, Trevor M. Harris, James Thompson, and J. Steven Kite, all of West Virginia University; William Kennedy (Dayton Society of Natural History); and Mark F. Seeman (Kent State University). Dr. J. Steven Kite deserves special note for engaging the author in an extended discussion of the geological history of the lower Scioto River Valley and its impact on archaeological preservation issues. 
Chapter 2. Journal Article Supplemental Information

S1. Variables used in GIS and statistical modeling

Table S1. Primary data sources used to create GIS layers

\begin{tabular}{|l|l|l|}
\hline Variable & $\begin{array}{l}\text { Original Type or } \\
\text { Resolution }\end{array}$ & Data Source \\
\hline Archaeology Components & Point & $\begin{array}{l}\text { Ohio Historic Preservation Office (OHPO), Ohio Archaeological } \\
\text { Inventory (OAI), obtained in 2014 }\end{array}$ \\
\hline DEM & $\begin{array}{l}\text { Raster Surface/ 1/3 arc } \\
\text { second, } 10 \mathrm{~m}^{2} \text { cell size }\end{array}$ & U.S. Geological Survey (U.S. Geological Survey, 2013) \\
\hline Soils & Raster, 10-m resolution & $\begin{array}{l}\text { U.S. Department of Agriculture (2017), gSSURGO Digital Soils } \\
\text { format }\end{array}$ \\
\hline Karst Landscape & Polygon & Tobin \& Weary, 2004 \\
\hline Physiographic Province & Polygon & based on Brockman, 2006 \\
\hline Wetlands & Polygon & National Wetlands Inventory (U.S. Fish \& Wildlife, 2017) \\
\hline Rivers & Polyline & $\begin{array}{l}\text { modified from U.S. Geological Survey (2017) against DEM } \\
\text { (U.S. Geological Survey, 2013) }\end{array}$ \\
\hline 2013 Population & Polygon c & United States Census Bureau (https://www.census.gov/data.html) \\
\hline
\end{tabular}

Additional Processing Notes:

All data was converted into the Ohio South state plane projected coordinate system:

NAD_1983_2011_StatePlane_Ohio_South_FIPS_3402. This coordinate system was based on NAD 1983 datum.

\section{S1.1 Variable Definitions and Maps}

Archaeology Component: Each Paleoindian component in this dataset was individually vetted by the author through consideration of both environmental data and data contained in site forms, unpublished reports, and published accounts. Early Archaic and Late Archaic components were not individually vetted by the author.

Distance to Wetland $(\mathrm{km})$ : Created layer from the National Wetlands Inventory (U.S. Fish \& Wildlife, 2017). This included merging two categories in the national inventory:

- Freshwater Emergent Wetland

- Freshwater Forested/Shrub Wetland

Distance to Large and Medium Rivers $(\mathrm{km})$ : River rankings were based on drainage area as reported in Childress (2001). Large rivers represent drainages of $>1000$ square miles (2590 sq. $\mathrm{km}$ ), whereas medium rivers have drainages between 200 and 999.9 square miles (518 - 2589 sq. $\mathrm{km})$.

$\begin{array}{ll}\text { Large River (all in miles } & \text { ): } \\ \text { Upper reach of Ohio } & >1000 \\ \text { Hocking } & 1197 \\ \text { G. Miami } & 3946 \\ \text { L. Miami } & 1757 \\ \text { Muskingum } & 8051\end{array}$




\begin{tabular}{|c|c|}
\hline Scioto & 6517 \\
\hline Whitewater & 1474 \\
\hline Paint Creek & 1144 \\
\hline Tuscarawas & 2590 \\
\hline Walhonding & 2256 \\
\hline Mahoning & 1140 \\
\hline \multicolumn{2}{|l|}{ Iedium River: } \\
\hline East Fork Whitewater & 382 \\
\hline Fourmile Creek & 315 \\
\hline Mad & 657 \\
\hline Stillwater & 676 \\
\hline East Fork Little Miami & 499 \\
\hline Ohio Brush Creek & 435 \\
\hline Salt Creek & 555 \\
\hline Deer Creek & 412 \\
\hline Big Darby Creek & 555 \\
\hline Big Walnut Creek & 557 \\
\hline Olentangy & 543 \\
\hline Symmes & 357 \\
\hline Raccoon Creek & 681 \\
\hline Moxahala Creek & 302 \\
\hline Licking River & 779 \\
\hline Wills Creek & 853 \\
\hline Stillwater Creek & 485 \\
\hline Sugar Creek & 357 \\
\hline Sandy Creek & 504 \\
\hline Killbuck Creek & 609 \\
\hline Little Muskingum River & 315 \\
\hline Little Beaver Creek & 503 \\
\hline
\end{tabular}

Large and medium river layers were created through modification of data from USGS Hydrography layer (U.S. Geological Survey, 2017) and DEM layer (U.S. Geological Survey, 2013).

Stream Convergence: Developed two classes for the junction of river systems:

- Large River Confluence Pt. (km) (e.g., Scioto to Ohio River)

- Large to Medium River Confluence Pt. (km) (e.g., Little Miami to Ohio River) 


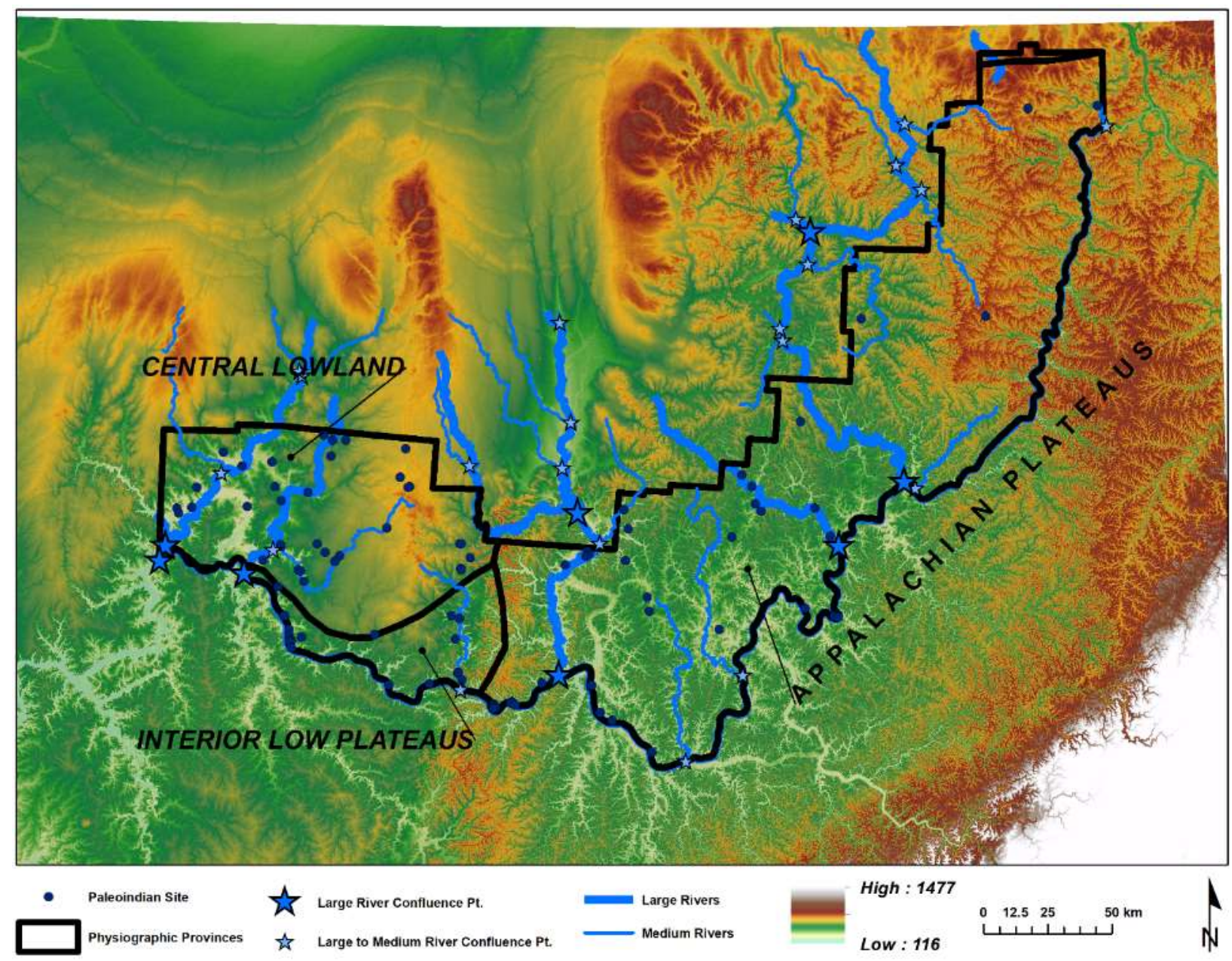

Figure S1. Study area showing large and medium rivers and large river confluence and large to medium river confluence points. Paleoindian sites used for modeling and physiographic boundaries also illustrated.

Soil Order: Soils data derived from gSSURGO raster (Natural Resources Conservation Service, 2017):

- Alfisols

- Entisols

- Inceptisols

- Mollisols

- Ultisols

Distance to Sand Dune $(\mathrm{km})$ : The sand dune layer was created from data contained within the soils layer. Sand dunes were defined by query definition of raster cells classified as Psamments; mapped as formed in either outwash, eolian deposits, or sandy loess for the parent material category; and mapped as either Lakin or Plainfield soil series. 
Distance to Trail $(\mathrm{km})$ : Layer derived by merging large and medium river polylines with Native American trails illustrated by Wilcox (1970) and digitized by William Kennedy Senior Curator at Dayton Society of Natural History.

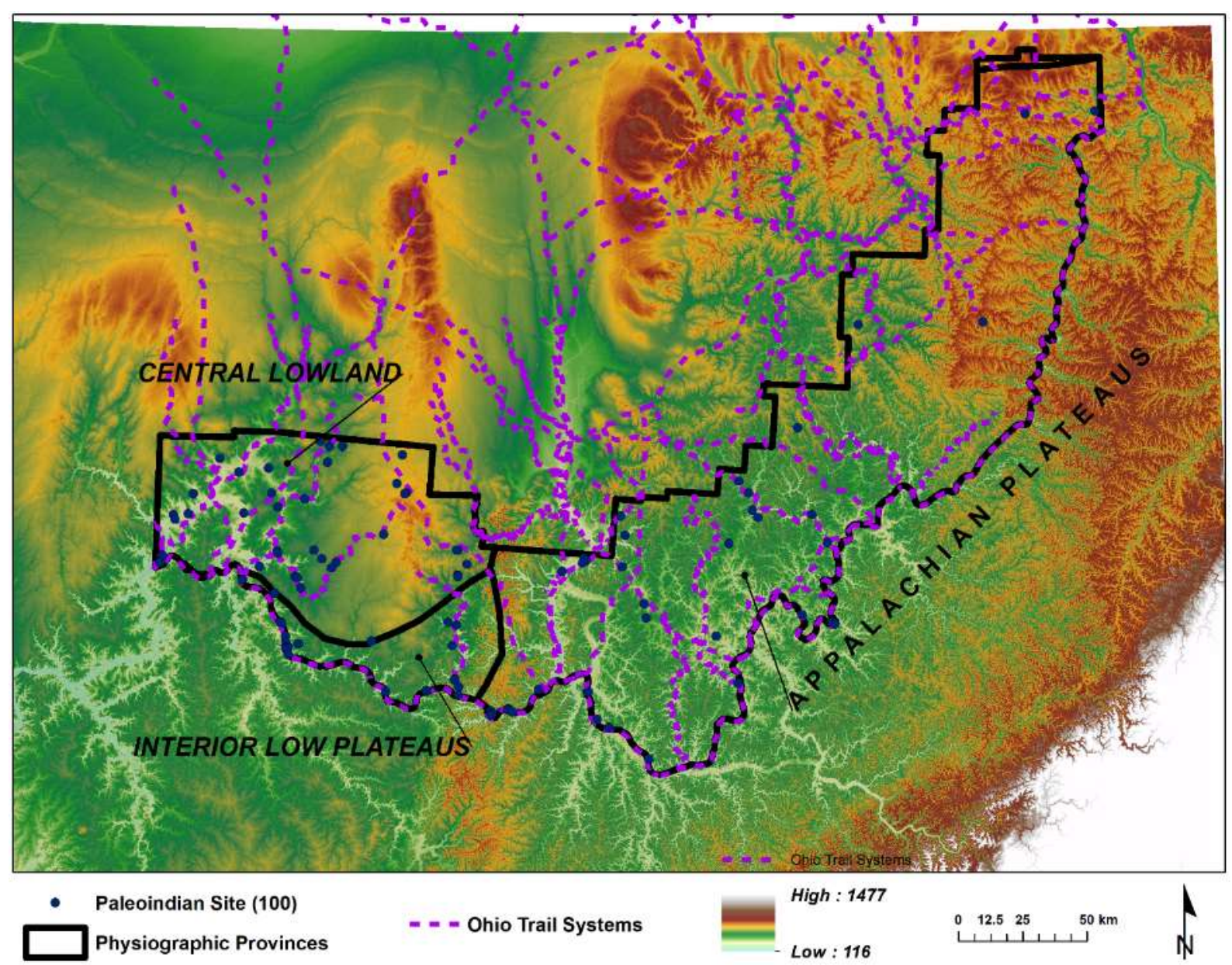

Figure S2. Study area showing potential trail systems used for modeling. Paleoindian sites used for modeling and physiographic boundaries also illustrated.

Distance to Karst Landscape ( $\mathrm{km}$ ): Karst landscape layer is derived from Tobin and Weary (2004) which was then clipped to the study area.

Viewshed (km2): Calculated using ArcMap 10.5 Viewshed tool with DEM layer.

Aspect: Compass direction of slope was calculated using ArcMap 10.5 Aspect tool and DEM layer. This variable was dichotomized and includes the following categories:

- North

- Northeast

- East

- Southeast $337.5-22.5^{\circ}$

$22.5-67.5^{\circ}$

$67.5-112.5^{\circ}$

$112.5-157.5^{\circ}$ 
- South

- Southwest

- West

- Northwest

- Flat $157.5-202.5^{\circ}$

$202.5-247.5^{\circ}$

$247.5-292.5^{\circ}$

$292.5-337.5^{\circ}$

-1 (insignificant amount of slope)

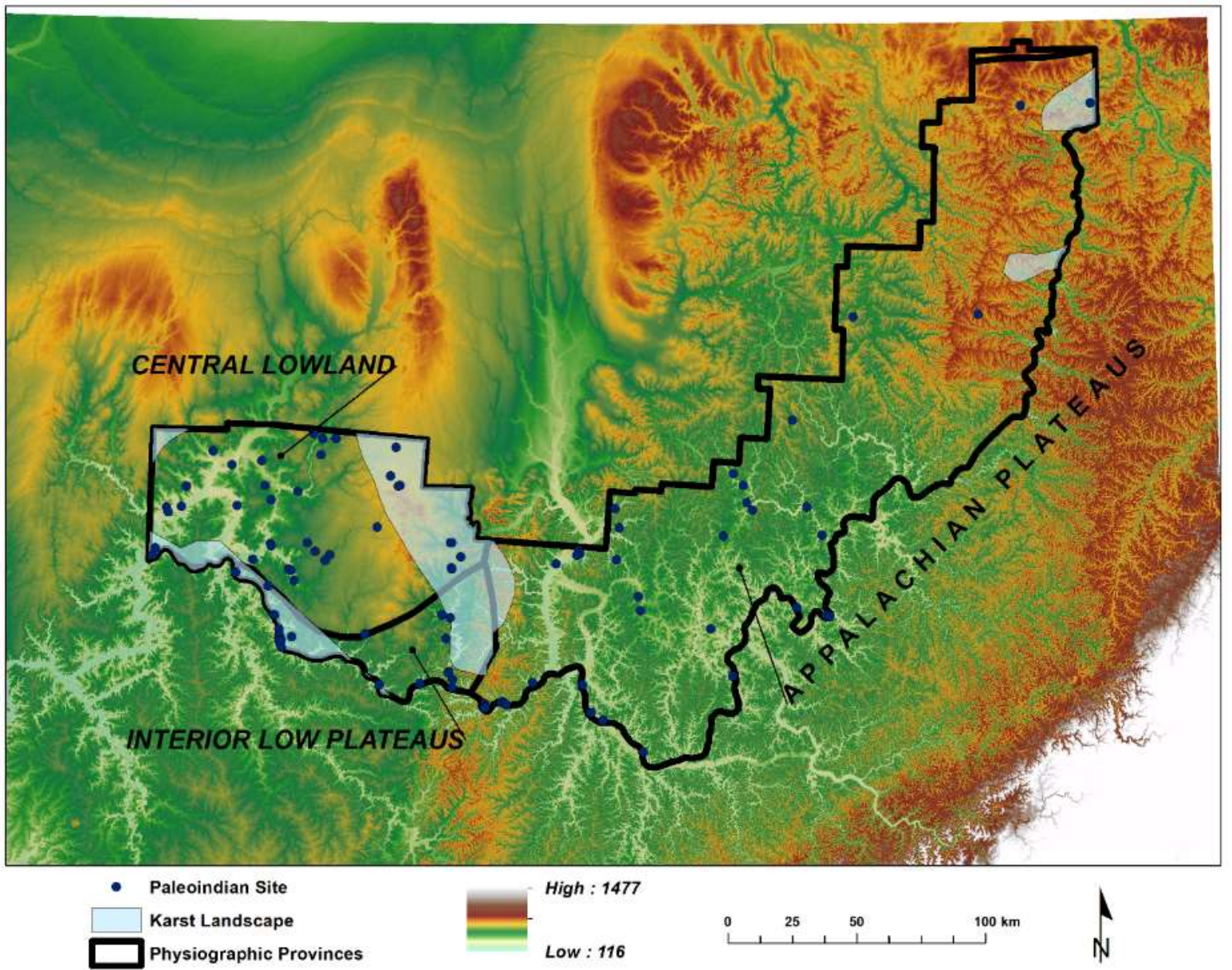

Figure S3. Study area showing karst landscape used for modeling. Paleoindian sites used for modeling and physiographic boundaries also illustrated.

Slope (m): Calculated using ArcMap 10.5 Slope tool with DEM layer.

Topographic Position Index (TPI): Based on Jenness (2006) ArcMap Spatial Analyst extension tools provided by Jenness Enterprises (http://www.jennessent.com/arcview/tpi.htm) and DEM layer. The following categories are defined:

- $1=$ Valley

- $2=$ Toe Slope

- $3=$ Flat Slope

- $4=$ Midslope 
- $5=$ Upper Slopes

- $6=$ Ridges

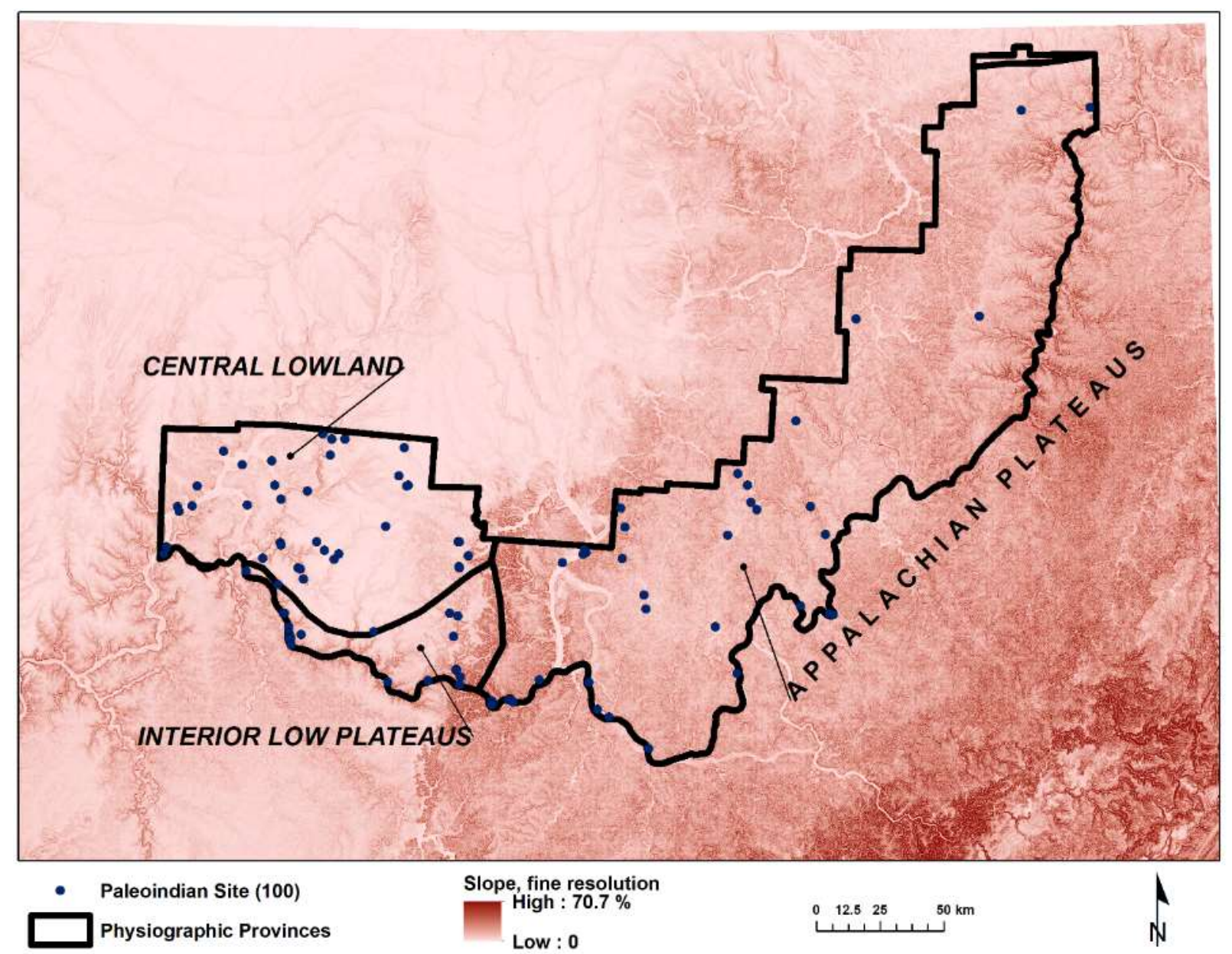

Figure S4. Study area showing slope percentage at fine resolution used for modeling. Paleoindian sites used for modeling and physiographic boundaries also illustrated.

Terrain Ruggedness Index (TRI): TRI is based on Riley et al. (1999) and is defined as the difference between the value of a DEM cell and the mean of an 8-cell neighborhood of surrounding cells. Ruggedness values follow Riley et al. (1999) and are as follows:

- Level

- Nearly Level

- Slightly Rugged

- Intermediately Rugged

- Moderately Rugged

- Highly Rugged

- Extremely Rugged
$0-80$ meters

81-116 meters

$117-161$ meters

$162-239$ meters

240-497 meters

498-958 meters

959-4367 meters 
Landform Curvature: In opposition to Slope, Landform Curvature represents the perpendicular axis to the direction of maximum slope. Calculated using ArgMap 0.5 Curvature tool with DEM layer.

- Positive values = increasing convexity perpendicular to direction of maximum slope

- Negative values $=$ increasing concavity perpendicular to direction of maximum slope

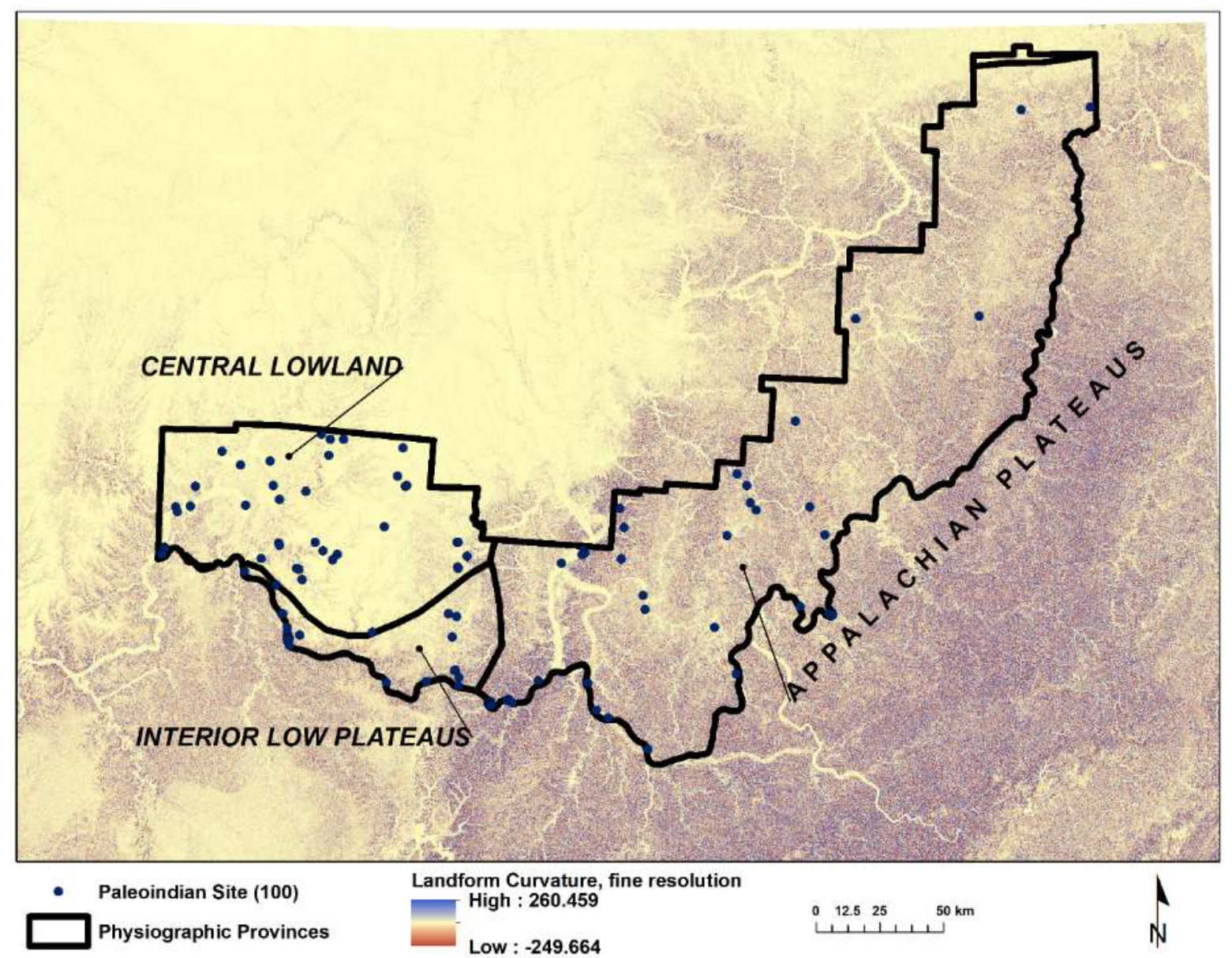

Figure S5. Study area showing landform curvature at fine resolution used for modeling. Paleoindian sites used for modeling and physiographic boundaries also illustrated. 
S1.2 Data results of statistical testing for Comprehensive, Cross-regional, and Cross-temporal GIS models

Table S2. Comprehensive model values and statistical results

\begin{tabular}{|c|c|c|c|c|c|c|}
\hline Variable & Measure & Paleoindian & Early Archaic & Late Archaic & Random Point & $\begin{array}{l}\text { Significance } \\
\text { Associations }\end{array}$ \\
\hline \multirow{2}{*}{$\begin{array}{l}\text { Distance to Large River } \\
\text { Confluence Pt. }(\mathrm{km})\end{array}$} & $\bar{x}$ & 35.66 & 40.50 & 40.53 & 46.93 & $\mathrm{p}<.05 ; \mathrm{a}$ \\
\hline & $s$ & 20.05 & 21.47 & 23.15 & 23.75 & - \\
\hline \multirow{3}{*}{$\begin{array}{l}\text { Distance to Large- } \\
\text { Medium River } \\
\text { Confluence Pt. }(\mathrm{km})\end{array}$} & $\bar{x}$ & 25.50 & 27.70 & 26.92 & 31.84 & $\mathrm{p}<.05 ; \mathrm{a}$ \\
\hline & & & & & & \\
\hline & $s$ & 13.96 & 12.94 & 13.98 & 13.09 & - \\
\hline \multirow{2}{*}{$\begin{array}{l}\text { Distance to Large River } \\
(\mathrm{km})\end{array}$} & $\bar{x}$ & 7.31 & 9.33 & 8.28 & 13.66 & $\mathbf{p}<.05 ; a, b, d$ \\
\hline & $s$ & 8.77 & 9.08 & 9.08 & 9.36 & - \\
\hline \multirow{2}{*}{$\begin{array}{l}\text { Distance to Medium } \\
\text { River (km) }\end{array}$} & $\bar{x}$ & 14.36 & 15.89 & 15.31 & 15.19 & $\mathrm{p}>.05$ \\
\hline & $s$ & 11.41 & 11.22 & 11.68 & 10.07 & - \\
\hline \multirow{2}{*}{$\begin{array}{l}\text { Distance to Wetland } \\
(\mathrm{km})\end{array}$} & $\bar{x}$ & 0.34 & 0.46 & 0.40 & 0.76 & $\mathrm{p}<.05 ; \mathrm{a}, \mathrm{c}$ \\
\hline & $s$ & 0.29 & 0.43 & 0.39 & 0.66 & - \\
\hline \multirow[t]{2}{*}{ Distance to Trail $(\mathrm{km})$} & $\bar{x}$ & 1.99 & 1.99 & 2.58 & 5.47 & $\mathbf{p}<.05 ;$ a, c \\
\hline & $s$ & 2.24 & 2.97 & 3.18 & 4.45 & - \\
\hline \multirow{2}{*}{$\begin{array}{l}\text { Distance to Sand Dune } \\
(\mathrm{km})\end{array}$} & $\bar{x}$ & 67.84 & 72.55 & 69.20 & 64.23 & $\mathrm{p}>.05$ \\
\hline & $s$ & 43.71 & 44.61 & 41.18 & 41.18 & - \\
\hline \multirow[t]{2}{*}{ TRI, Fine Scale } & $\bar{x}$ & 57.5 & 59.8 & 62.8 & 106.1 & $\mathrm{p}<.05 ; \mathrm{a}$ \\
\hline & $s$ & 31.8 & 36.4 & 38.2 & 44.6 & - \\
\hline \multirow[t]{2}{*}{ TRI, Medium Scale } & $\bar{x}$ & 137.9 & 134.8 & 141.2 & 185.4 & $\mathrm{p}<.05 ; \mathrm{a}, \mathrm{d}$ \\
\hline & $s$ & 51.0 & 51.9 & 55.7 & 56.3 & - \\
\hline \multirow[t]{2}{*}{ TRI, Coarse Scale } & $\bar{x}$ & 283.6 & 271.0 & 281.0 & 285.0 & $\mathrm{p}<.05 ; \mathrm{a}, \mathrm{d}$ \\
\hline & $s$ & 76.5 & 59.6 & 63.5 & 48.3 & - \\
\hline \multirow{2}{*}{$\begin{array}{l}\text { Landform Curvature, } \\
\text { Fine }\end{array}$} & $\bar{x}$ & 3.0 & 0.6 & 0.2 & -1.3 & p<.05; b, c \\
\hline & $s$ & 12.3 & 13.3 & 13.3 & 27.3 & - \\
\hline \multirow{2}{*}{$\begin{array}{l}\text { Landform Curvature, } \\
\text { Medium }\end{array}$} & $\bar{x}$ & -4.3 & -10.3 & -9.0 & -0.8 & $\mathrm{p}>.05$ \\
\hline & $s$ & 43.1 & 43.5 & 50.3 & 62.2 & \\
\hline \multirow{2}{*}{$\begin{array}{l}\text { Landform Curvature, } \\
\text { Coarse }\end{array}$} & $\bar{x}$ & -24.5 & -19.6 & -19.7 & -4.0 & $\mathbf{p}<.05 ; \mathbf{a}$ \\
\hline & $s$ & 48.7 & 55.5 & 60.4 & 69.9 & - \\
\hline \multirow[t]{2}{*}{ Elevation (m) } & $\bar{x}$ & 207.6 & 228.5 & 219.9 & 274.5 & $\mathrm{p}<.05 ; \mathrm{a}, \mathrm{b}, \mathrm{c}, \mathrm{d}$ \\
\hline & $s$ & 55.7 & 59.7 & 60.6 & 53.8 & - \\
\hline \multirow{2}{*}{$\begin{array}{l}\text { Distance to Karst } \\
\text { Landscape }(\mathrm{km})\end{array}$} & $\bar{x}$ & 25.02 & 30.24 & 30.93 & 38.75 & $\mathrm{p}<.05 ; \mathrm{a}, \mathrm{c}$ \\
\hline & $s$ & 33.53 & 35.27 & 35.65 & 34.82 & - \\
\hline \multirow[t]{2}{*}{ Slope, Fine } & $\bar{x}$ & 3.2 & 2.9 & 3.1 & 5.8 & $\mathrm{p}<.05 ; \mathbf{a}$ \\
\hline & $s$ & 3.3 & 2.9 & 3.2 & 4.7 & - \\
\hline \multirow[t]{2}{*}{ Slope, Medium } & $\bar{x}$ & 1.7 & 1.4 & 1.6 & 1.3 & $\mathrm{p}<.05 ; \mathrm{a}, \mathrm{d}$ \\
\hline & $s$ & 1.3 & 1.2 & 1.2 & 1.0 & - \\
\hline Slope, Coarse & $\bar{x}$ & 0.2 & 0.2 & 0.2 & 0.1 & $\mathrm{p}>.05$ \\
\hline
\end{tabular}




\begin{tabular}{|c|c|c|c|c|c|c|}
\hline Variable & Measure & Paleoindian & Early Archaic & Late Archaic & Random Point & $\begin{array}{l}\text { Significance } \\
\text { Associations }\end{array}$ \\
\hline & $s$ & 0.1 & 0.1 & 0.1 & 0.1 & - \\
\hline \multirow[t]{2}{*}{ Viewshed (km2) } & $\bar{x}$ & 499.6 & 660.6 & 480.7 & 893.7 & $\mathbf{p}<.05 ; \mathbf{a}$ \\
\hline & $s$ & 1908.6 & 2018.7 & 1663.6 & 2277.5 & - \\
\hline Aspect, East & - & $10.00 \%$ & $8.08 \%$ & $6.77 \%$ & $11.54 \%$ & $\mathrm{p}>.05$ \\
\hline Aspect, Flat & - & $34.00 \%$ & $35.22 \%$ & $33.65 \%$ & $13.53 \%$ & $\mathbf{p}<.05 ; \mathbf{a}$ \\
\hline Aspect, North & - & $8.00 \%$ & $7.43 \%$ & $7.30 \%$ & $6.98 \%$ & $\mathrm{p}>.05$ \\
\hline Aspect, Northeast & - & $4.00 \%$ & $6.46 \%$ & $7.62 \%$ & $8.40 \%$ & $\mathrm{p}>.05$ \\
\hline Aspect, Northwest & - & $10.00 \%$ & $9.69 \%$ & $7.41 \%$ & $11.68 \%$ & $\mathrm{p}>.05$ \\
\hline Aspect, South & - & $5.00 \%$ & $7.75 \%$ & $8.68 \%$ & $12.11 \%$ & $\mathbf{p}<.05 ; \mathbf{a}$ \\
\hline Aspect, Southeast & - & $8.00 \%$ & $8.40 \%$ & $8.25 \%$ & $10.97 \%$ & $\mathrm{p}>.05$ \\
\hline Aspect, Southwest & - & $11.00 \%$ & $7.92 \%$ & $10.69 \%$ & $11.82 \%$ & $\mathrm{p}>.05$ \\
\hline Aspect, West & - & $10.00 \%$ & $9.05 \%$ & $9.63 \%$ & $12.96 \%$ & $\mathrm{p}>.05$ \\
\hline TPI, Fine, flat & - & $33.00 \%$ & $38.45 \%$ & $35.24 \%$ & $15.81 \%$ & $\mathbf{p}<.05 ; \mathbf{a}$ \\
\hline TPI, Fine, midslope & - & $4.00 \%$ & $2.91 \%$ & $4.13 \%$ & $6.55 \%$ & $\mathrm{p}>.05$ \\
\hline TPI, Fine, ridge & - & $19.00 \%$ & $17.29 \%$ & $17.57 \%$ & $29.91 \%$ & $\mathbf{p}<.05 ; \mathbf{a}$ \\
\hline TPI, Fine, toe slope & - & $14.00 \%$ & $10.66 \%$ & $10.48 \%$ & $7.26 \%$ & $\mathbf{p}<.05$; a \\
\hline TPI, Fine, upper slope & - & $13.00 \%$ & $11.95 \%$ & $10.69 \%$ & $7.69 \%$ & $\mathrm{p}>.05$ \\
\hline TPI, Fine, valley & - & $17.00 \%$ & $18.74 \%$ & $21.90 \%$ & $32.76 \%$ & $\mathbf{p}<.05 ; \mathbf{a}$ \\
\hline TPI, Medium, flat & - & $12.00 \%$ & $7.43 \%$ & $7.20 \%$ & $5.56 \%$ & $\mathbf{p}<.05 ; \mathbf{a}$ \\
\hline TPI, Medium, midslope & - & $0.00 \%$ & $0.81 \%$ & $1.06 \%$ & $1.99 \%$ & $\mathrm{p}>.05$ \\
\hline TPI, Medium, ridge & - & $26.00 \%$ & $32.79 \%$ & $30.16 \%$ & $44.59 \%$ & $\mathbf{p}<.05 ; \mathbf{a}$ \\
\hline TPI, Medium, toe slope & - & $2.00 \%$ & $4.52 \%$ & $5.40 \%$ & $1.57 \%$ & $\mathrm{p}>.05$ \\
\hline $\begin{array}{l}\text { TPI, Medium, upper } \\
\text { slope }\end{array}$ & - & $7.00 \%$ & $4.36 \%$ & $3.81 \%$ & $2.28 \%$ & $\mathbf{p}<.05 ; \mathbf{a}$ \\
\hline TPI, Medium, valley & - & $53.00 \%$ & $50.08 \%$ & $52.38 \%$ & $44.02 \%$ & $\mathrm{p}>.05$ \\
\hline TPI, Coarse, flat & - & $2.00 \%$ & $0.81 \%$ & $0.53 \%$ & $0.85 \%$ & $\mathrm{p}>.05$ \\
\hline TPI, Coarse, midslope & - & $1.00 \%$ & $0.00 \%$ & $0.21 \%$ & $1.42 \%$ & $\mathrm{p}>.05$ \\
\hline TPI, Coarse, ridge & - & $10.00 \%$ & $21.81 \%$ & $16.61 \%$ & $52.71 \%$ & $\mathrm{p}<.05 ; \mathrm{a}, \mathrm{b}, \mathrm{d}$ \\
\hline TPI, Coarse, toe slope & - & $1.00 \%$ & $0.32 \%$ & $0.42 \%$ & $0.71 \%$ & $\mathrm{p}>.05$ \\
\hline TPI, Coarse, upper slope & - & $1.00 \%$ & $0.48 \%$ & $0.32 \%$ & $0.85 \%$ & $\mathrm{p}>.05$ \\
\hline TPI, Coarse, valley & - & $85.00 \%$ & $76.58 \%$ & $81.90 \%$ & $43.45 \%$ & $\mathrm{p}<.05 ; \mathrm{a}, \mathrm{d}$ \\
\hline Soil Order, Alfisols & - & $73.74 \%$ & $66.49 \%$ & $64.62 \%$ & $56.89 \%$ & $\mathbf{p}<.05 ; \mathbf{a}, \mathbf{b}, \mathbf{c}$ \\
\hline Soil Order, Entisols & - & $3.03 \%$ & $7.05 \%$ & $9.02 \%$ & $7.04 \%$ & $\mathrm{p}>.05$ \\
\hline Soil Order, Inceptisols & - & $8.08 \%$ & $10.93 \%$ & $12.72 \%$ & $13.34 \%$ & $\mathrm{p}>.05$ \\
\hline Soil Order, Mollisols & - & $11.11 \%$ & $11.99 \%$ & $10.52 \%$ & $2.64 \%$ & $\mathbf{p}<.05 ; \mathbf{a}$ \\
\hline Soil Order, Ultisols & - & $4.04 \%$ & $3.53 \%$ & $3.12 \%$ & $20.09 \%$ & $\mathbf{p}<.05$; a \\
\hline $\begin{array}{l}\text { Province, Appalachian } \\
\text { Plateaus }\end{array}$ & - & $39.00 \%$ & $43.46 \%$ & $49.10 \%$ & $69.66 \%$ & $\mathrm{p}<.05 ; \mathrm{a}, \mathrm{d}$ \\
\hline $\begin{array}{l}\text { Province, Central } \\
\text { Lowland }\end{array}$ & - & $38.00 \%$ & $48.30 \%$ & $37.35 \%$ & $24.50 \%$ & $\mathrm{p}<.05 ; \mathrm{a}, \mathrm{d}$ \\
\hline $\begin{array}{l}\text { Province, Interior Low } \\
\text { Plateaus }\end{array}$ & - & $23.00 \%$ & $8.24 \%$ & $13.54 \%$ & $5.84 \%$ & $\mathbf{p}<.05 ; a, b, c, d$ \\
\hline
\end{tabular}


d = Early Archaic versus Late Archaic

Table S3. Appalachian Plateaus model values and statistical results

\begin{tabular}{|c|c|c|c|c|c|c|}
\hline Variable & Measure & Paleoindian & Early Archaic & Late Archaic & Random Point & $\begin{array}{l}\text { Significance } \\
\text { Associations }\end{array}$ \\
\hline \multirow{2}{*}{$\begin{array}{l}\text { Distance to Large River } \\
\text { Confluence Pt. (km) }\end{array}$} & $\bar{x}$ & 44.08 & 38.97 & 42.16 & 49.73 & $\mathbf{p}<.05$; \\
\hline & $s$ & 22.05 & 25.63 & 28.56 & 26.05 & - \\
\hline \multirow{2}{*}{$\begin{array}{l}\text { Distance to Large- } \\
\text { Medium River } \\
\text { Confluence Pt. }(\mathrm{km})\end{array}$} & $\bar{x}$ & 26.06 & 32.82 & 30.59 & 33.08 & $\mathrm{p}>.05$ \\
\hline & $s$ & 17.30 & 14.77 & 15.03 & 13.67 & - \\
\hline \multirow{2}{*}{$\begin{array}{l}\text { Distance to Large River } \\
(\mathrm{km})\end{array}$} & $\bar{x}$ & 8.84 & 8.22 & 30.66 & 13.87 & $\mathbf{p}<.05 ; \mathbf{a}$ \\
\hline & $s$ & 9.47 & 9.34 & 15.01 & 9.40 & - \\
\hline \multirow{2}{*}{$\begin{array}{l}\text { Distance to Medium } \\
\text { River }(\mathrm{km})\end{array}$} & $\bar{x}$ & 16.43 & 19.11 & 17.86 & 15.69 & $\mathrm{p}>.05$ \\
\hline & $s$ & 12.08 & 12.78 & 12.87 & 9.97 & - \\
\hline \multirow{2}{*}{$\begin{array}{l}\text { Distance to Wetland } \\
(\mathrm{km})\end{array}$} & $\bar{x}$ & 0.42 & 0.50 & 0.44 & 0.77 & $\mathbf{p}<.05 ; \mathbf{a}$ \\
\hline & $s$ & 0.38 & 0.47 & 0.43 & 0.67 & - \\
\hline \multirow[t]{2}{*}{ Distance to Trail $(\mathrm{km})$} & $\bar{x}$ & 1.63 & 2.74 & 2.49 & 5.43 & $\mathbf{p}<.05$; a \\
\hline & $s$ & 1.77 & 3.66 & 3.32 & 4.52 & - \\
\hline \multirow{2}{*}{$\begin{array}{l}\text { Distance to Sand Dune } \\
(\mathrm{km})\end{array}$} & $\bar{x}$ & 37.30 & 41.35 & 46.11 & 74.45 & $\mathbf{p}<.05 ; \mathbf{a}$ \\
\hline & $s$ & 35.15 & 37.68 & 42.49 & 43.52 & - \\
\hline \multirow[t]{2}{*}{ TRI, Fine Scale } & $\bar{x}$ & 55.6 & 71.1 & 68.6 & 106.2 & $\mathrm{p}<.05 ; \mathrm{a}, \mathrm{b}$ \\
\hline & $s$ & 30.7 & 43.1 & 42.9 & 45.5 & - \\
\hline \multirow[t]{2}{*}{ TRI, Medium Scale } & $\bar{x}$ & 141.1 & 153.5 & 154.0 & 185.7 & $\mathbf{p}<.05$; \\
\hline & $s$ & 60.0 & 58.2 & 60.8 & 57.0 & - \\
\hline \multirow[t]{2}{*}{ TRI, Coarse Scale } & $\bar{x}$ & 294.1 & 290.4 & 293.9 & 287.6 & $\mathrm{p}>.05$ \\
\hline & $s$ & 48.2 & 42.4 & 41.6 & 47.4 & - \\
\hline \multirow{2}{*}{$\begin{array}{l}\text { Landform Curvature, } \\
\text { Fine }\end{array}$} & $\bar{x}$ & 0.4 & 1.9 & 1.0 & -0.6 & $\mathrm{p}>.05$ \\
\hline & $s$ & 9.1 & 18.1 & 16.3 & 27.0 & - \\
\hline \multirow{2}{*}{$\begin{array}{l}\text { Landform Curvature, } \\
\text { Medium }\end{array}$} & $\bar{x}$ & -4.6 & -7.8 & -7.9 & -4.4 & $\mathrm{p}>.05$ \\
\hline & $s$ & 42.5 & 48.7 & 55.5 & 58.5 & - \\
\hline \multirow{2}{*}{$\begin{array}{l}\text { Landform Curvature, } \\
\text { Coarse }\end{array}$} & $\bar{x}$ & -27.3 & -23.0 & -22.7 & -5.1 & $\mathbf{p}<.05 ; \mathbf{a}$ \\
\hline & $s$ & 47.7 & 64.1 & 61.5 & 67.1 & - \\
\hline \multirow[t]{2}{*}{ Elevation (m) } & $\bar{x}$ & 196.7 & 217.4 & 219.1 & 286.4 & $\mathbf{p}<.05 ; \mathbf{a}, \mathbf{b}$ \\
\hline & $s$ & 45.8 & 61.2 & 65.7 & 55.2 & - \\
\hline \multirow{2}{*}{$\begin{array}{l}\text { Distance to Karst } \\
\text { Landscape }(\mathrm{km})\end{array}$} & $\bar{x}$ & 52.47 & 56.73 & 53.19 & 39.30 & $\mathbf{p}<.05 ;$ a \\
\hline & $s$ & 39.19 & 38.58 & 38.98 & 34.69 & - \\
\hline \multirow[t]{2}{*}{ Slope, Fine } & $\bar{x}$ & 3.3 & 3.8 & 3.8 & 5.5 & $\mathbf{p}<.05 ;$ a \\
\hline & $s$ & 3.7 & 3.4 & 3.7 & 4.4 & - \\
\hline \multirow[t]{2}{*}{ Slope, Medium } & $\bar{x}$ & 1.9 & 1.8 & 1.8 & 1.3 & $\mathbf{p}<.05 ;$ a \\
\hline & $s$ & 1.4 & 1.3 & 1.3 & 0.9 & - \\
\hline \multirow[t]{2}{*}{ Slope, Coarse } & $\bar{x}$ & 0.1 & 0.1 & 0.1 & 0.1 & $p<.05 ; d$ \\
\hline & $s$ & 0.1 & 0.1 & 0.1 & 0.1 & - \\
\hline Viewshed (km2) & $\bar{x}$ & 133.5 & 208.0 & 233.2 & 832.6 & $\mathrm{p}>.05$ \\
\hline
\end{tabular}




\begin{tabular}{|c|c|c|c|c|c|c|}
\hline Variable & Measure & Paleoindian & Early Archaic & Late Archaic & Random Point & $\begin{array}{c}\text { Significance } \\
\text { Associations }\end{array}$ \\
\hline & $s$ & 327.3 & 911.4 & 939.6 & 2248.4 & - \\
\hline Aspect, East & - & $10.26 \%$ & $8.92 \%$ & $6.47 \%$ & $11.25 \%$ & $\mathrm{p}>.05$ \\
\hline Aspect, Flat & - & $35.90 \%$ & $31.97 \%$ & $33.84 \%$ & $13.91 \%$ & $\mathrm{p}<.05 ; \mathrm{a}$ \\
\hline Aspect, North & - & $7.69 \%$ & $5.95 \%$ & $6.47 \%$ & $7.16 \%$ & $\mathrm{p}>.05$ \\
\hline Aspect, Northeast & - & $5.13 \%$ & $8.18 \%$ & $7.11 \%$ & $8.38 \%$ & $\mathrm{p}>.05$ \\
\hline Aspect, Northwest & - & $5.13 \%$ & $9.67 \%$ & $5.82 \%$ & $12.07 \%$ & $\mathrm{p}>.05$ \\
\hline Aspect, South & - & $7.69 \%$ & $8.92 \%$ & $9.91 \%$ & $12.68 \%$ & $\mathrm{p}>.05$ \\
\hline Aspect, Southeast & - & $7.69 \%$ & $8.92 \%$ & $9.70 \%$ & $11.86 \%$ & $\mathrm{p}>.05$ \\
\hline Aspect, Southwest & - & $10.26 \%$ & $7.81 \%$ & $10.56 \%$ & $10.63 \%$ & $\mathrm{p}>.05$ \\
\hline Aspect, West & - & $10.26 \%$ & $9.67 \%$ & $10.13 \%$ & $12.07 \%$ & $\mathrm{p}>.05$ \\
\hline TPI, Fine, flat & - & $35.90 \%$ & $27.14 \%$ & $29.96 \%$ & $17.59 \%$ & $\mathbf{p}<.05 ; \mathbf{a}$ \\
\hline TPI, Fine, midslope & - & $2.56 \%$ & $5.20 \%$ & $4.96 \%$ & $5.32 \%$ & $\mathrm{p}>.05$ \\
\hline TPI, Fine, ridge & - & $17.95 \%$ & $26.77 \%$ & $21.12 \%$ & $30.67 \%$ & $\mathrm{p}>.05$ \\
\hline TPI, Fine, toe slope & - & $12.82 \%$ & $10.78 \%$ & $12.07 \%$ & $7.98 \%$ & $\mathrm{p}>.05$ \\
\hline TPI, Fine, upper slope & - & $10.26 \%$ & $8.55 \%$ & $9.48 \%$ & $7.57 \%$ & $\mathrm{p}>.05$ \\
\hline TPI, Fine, valley & - & $20.51 \%$ & $21.56 \%$ & $22.41 \%$ & $30.88 \%$ & $\mathrm{p}>.05$ \\
\hline TPI, Medium, flat & - & $17.95 \%$ & $3.35 \%$ & $5.60 \%$ & $6.13 \%$ & $\mathrm{p}<.05 ; \mathrm{a}, \mathrm{b}, \mathrm{c}$ \\
\hline TPI, Medium, midslope & - & $0.00 \%$ & $0.74 \%$ & $1.29 \%$ & $1.84 \%$ & $\mathrm{p}>.05$ \\
\hline TPI, Medium, ridge & - & $17.95 \%$ & $39.78 \%$ & $34.70 \%$ & $43.56 \%$ & $\mathrm{p}<.05 ; \mathrm{a}, \mathrm{b}, \mathrm{c}$ \\
\hline TPI, Medium, toe slope & - & $2.56 \%$ & $2.97 \%$ & $4.09 \%$ & $1.84 \%$ & $\mathrm{p}>.05$ \\
\hline $\begin{array}{l}\text { TPI, Medium, upper } \\
\text { slope }\end{array}$ & - & $10.26 \%$ & $2.97 \%$ & $3.45 \%$ & $2.04 \%$ & $\mathrm{p}<.05 ; \mathrm{a}$ \\
\hline TPI, Medium, valley & - & $51.28 \%$ & $50.19 \%$ & $50.86 \%$ & $44.58 \%$ & $\mathrm{p}>.05$ \\
\hline TPI, Coarse, flat & - & $0.00 \%$ & $0.00 \%$ & $0.22 \%$ & $1.23 \%$ & $\mathrm{p}>.05$ \\
\hline TPI, Coarse, midslope & - & $0.00 \%$ & $0.00 \%$ & $0.00 \%$ & $1.64 \%$ & $\mathrm{p}>.05$ \\
\hline TPI, Coarse, ridge & - & $5.13 \%$ & $17.47 \%$ & $13.36 \%$ & $50.31 \%$ & $\mathrm{p}<.05 ; \mathrm{a}$ \\
\hline TPI, Coarse, toe slope & - & $0.00 \%$ & $0.37 \%$ & $0.43 \%$ & $0.41 \%$ & $\mathrm{p}>.05$ \\
\hline $\begin{array}{l}\text { TPI, Coarse, upper } \\
\text { slope }\end{array}$ & - & $0.00 \%$ & $0.00 \%$ & $0.22 \%$ & $1.23 \%$ & $\mathrm{p}>.05$ \\
\hline TPI, Coarse, valley & - & $94.87 \%$ & $82.16 \%$ & $85.78 \%$ & $45.19 \%$ & $\mathbf{p}<.05 ; \mathrm{a}$ \\
\hline Soil Order, Alfisols & - & $79.49 \%$ & $67.86 \%$ & $67.13 \%$ & $59.83 \%$ & $\mathrm{p}<.05 ; \mathrm{a}, \mathrm{c}$ \\
\hline Soil Order, Entisols & - & $5.13 \%$ & $5.95 \%$ & $7.41 \%$ & $8.67 \%$ & $\mathrm{p}>.05$ \\
\hline Soil Order, Inceptisols & - & $7.69 \%$ & $17.86 \%$ & $18.75 \%$ & $12.90 \%$ & $\mathrm{p}>.05$ \\
\hline Soil Order, Mollisols & - & $0.00 \%$ & $0.40 \%$ & $0.93 \%$ & $3.59 \%$ & $\mathrm{p}>.05$ \\
\hline Soil Order, Ultisols & - & $7.69 \%$ & $7.94 \%$ & $5.79 \%$ & $15.01 \%$ & $\mathrm{p}>.05$ \\
\hline
\end{tabular}

$\mathrm{a}=$ Paleoindian versus Random Point

$\mathrm{b}=$ Paleoindian versus Early Archaic

$\mathrm{c}=$ Paleoindian versus Late Archaic

$\mathrm{d}=$ Early Archaic versus Late Archaic 
Table S4. Central Lowlands model values and statistical results

\begin{tabular}{|c|c|c|c|c|c|c|}
\hline Variable & Measure & Paleoindian & Early Archaic & Late Archaic & Random Point & $\begin{array}{l}\text { Significance } \\
\text { Associations }\end{array}$ \\
\hline \multirow{2}{*}{$\begin{array}{l}\text { Distance to Large River } \\
\text { Confluence Pt. }(\mathrm{km})\end{array}$} & $\bar{x}$ & 38.38 & 42.02 & 38.93 & 39.02 & $\mathrm{p}>.05$ \\
\hline & $s$ & 19.75 & 18.52 & 17.71 & 15.16 & - \\
\hline \multirow{3}{*}{$\begin{array}{l}\text { Distance to Large- } \\
\text { Medium River } \\
\text { Confluence Pt. }(\mathrm{km})\end{array}$} & $\bar{x}$ & 23.48 & 23.69 & 22.56 & 31.09 & $\mathrm{p}<.05 ; \mathrm{a}$ \\
\hline & & 1056 & 891 & & 1052 & - \\
\hline & & & & & & \\
\hline \multirow{2}{*}{$\begin{array}{l}\text { Distance to Large River } \\
(\mathrm{km})\end{array}$} & $\bar{x}$ & 10.43 & 11.04 & 10.10 & 14.45 & $\mathbf{p}<.05 ; \mathbf{a}$ \\
\hline & $s$ & 8.83 & 8.70 & 8.81 & 9.51 & - \\
\hline \multirow{2}{*}{$\begin{array}{l}\text { Distance to Medium } \\
\text { River }(\mathrm{km})\end{array}$} & $\bar{x}$ & 13.23 & 13.53 & 12.81 & 14.80 & $\mathrm{p}>.05$ \\
\hline & $s$ & 11.06 & 9.14 & 9.68 & 10.84 & - \\
\hline \multirow{2}{*}{$\begin{array}{l}\text { Distance to Wetland } \\
(\mathrm{km})\end{array}$} & $\bar{x}$ & 0.32 & 0.46 & 0.41 & 0.69 & $p<.05 ; a, d$ \\
\hline & $s$ & 0.23 & 0.41 & 0.37 & 0.61 & - \\
\hline \multirow[t]{2}{*}{ Distance to Trail $(\mathrm{km})$} & $\bar{x}$ & 2.91 & 1.31 & 3.14 & 5.68 & $\mathbf{p}<.05 ; a, b, d$ \\
\hline & $s$ & 2.78 & 1.92 & 3.23 & 4.48 & - \\
\hline \multirow{2}{*}{$\begin{array}{l}\text { Distance to Sand Dune } \\
(\mathrm{km})\end{array}$} & $\bar{x}$ & 102.87 & 104.59 & 106.03 & 45.18 & $\mathbf{p}<.05 ; a$ \\
\hline & $s$ & 28.27 & 26.52 & 27.81 & 21.00 & - \\
\hline \multirow[t]{2}{*}{ TRI, Fine Scale } & $\bar{x}$ & 57.1 & 50.6 & 54.7 & 99.7 & $\mathrm{p}<.05 ; \mathrm{a}$ \\
\hline & $s$ & 30.1 & 27.3 & 28.8 & 41.1 & - \\
\hline \multirow[t]{2}{*}{ TRI, Medium Scale } & $\bar{x}$ & 122.4 & 113.5 & 117.3 & 178.3 & $\mathrm{p}<.05 ; \mathrm{a}$ \\
\hline & $s$ & 43.5 & 34.1 & 37.0 & 56.7 & - \\
\hline \multirow[t]{2}{*}{ TRI, Coarse Scale } & $\bar{x}$ & 248.6 & 243.1 & 245.3 & 278.7 & $\mathrm{p}<.05 ; \mathrm{a}$ \\
\hline & $s$ & 47.2 & 41.6 & 36.6 & 53.8 & - \\
\hline \multirow{2}{*}{$\begin{array}{l}\text { Landform Curvature, } \\
\text { Fine }\end{array}$} & $\bar{x}$ & 4.9 & -0.5 & -0.8 & -2.0 & p<.05; b, c \\
\hline & $s$ & 13.5 & 7.1 & 7.7 & 25.7 & - \\
\hline \multirow{2}{*}{$\begin{array}{l}\text { Landform Curvature, } \\
\text { Medium }\end{array}$} & $\bar{x}$ & -0.6 & -10.4 & -9.1 & 10.8 & $\mathrm{p}>.05$ \\
\hline & $s$ & 48.0 & 32.0 & 35.2 & 66.7 & - \\
\hline \multirow{2}{*}{$\begin{array}{l}\text { Landform Curvature, } \\
\text { Coarse }\end{array}$} & $\bar{x}$ & -5.9 & -10.5 & -8.2 & -2.3 & $\mathrm{p}>.05$ \\
\hline & $s$ & 30.0 & 33.2 & 41.7 & 76.0 & - \\
\hline \multirow[t]{2}{*}{ Elevation (m) } & $\bar{x}$ & 238.6 & 246.5 & 234.3 & 250.2 & $\mathrm{p}<.05 ; \mathrm{d}$ \\
\hline & $s$ & 60.8 & 53.5 & 53.3 & 37.4 & - \\
\hline \multirow{2}{*}{$\begin{array}{l}\text { Distance to Karst } \\
\text { Landscape }(\mathrm{km})\end{array}$} & $\bar{x}$ & 11.09 & 11.17 & 12.21 & 31.30 & $\mathrm{p}<.05 ; \mathrm{a}$ \\
\hline & $s$ & 9.45 & 10.33 & 9.82 & 34.31 & - \\
\hline \multirow[t]{2}{*}{ Slope, Fine } & $\bar{x}$ & 2.9 & 2.0 & 2.2 & 6.2 & $\mathrm{p}<.05 ; \mathrm{a}, \mathrm{b}$ \\
\hline & $s$ & 3.1 & 2.0 & 2.3 & 5.2 & - \\
\hline \multirow[t]{2}{*}{ Slope, Medium } & $\bar{x}$ & 1.1 & 0.9 & 1.1 & 1.4 & $\mathrm{p}>.05$ \\
\hline & $s$ & 0.8 & 0.7 & 0.8 & 1.1 & - \\
\hline \multirow[t]{2}{*}{ Slope, Coarse } & $\bar{x}$ & 0.2 & 0.2 & 0.2 & 0.2 & $\mathrm{p}<.05 ; \mathrm{a}$ \\
\hline & $s$ & 0.1 & 0.1 & 0.1 & 0.1 & - \\
\hline \multirow[t]{2}{*}{ Viewshed (km2) } & $\bar{x}$ & 1090.2 & 1139.1 & 914.1 & 1193.3 & $p<.05 ; \mathrm{d}$ \\
\hline & $s$ & 3000.0 & 2680.6 & 2424.7 & 2525.4 & - \\
\hline
\end{tabular}




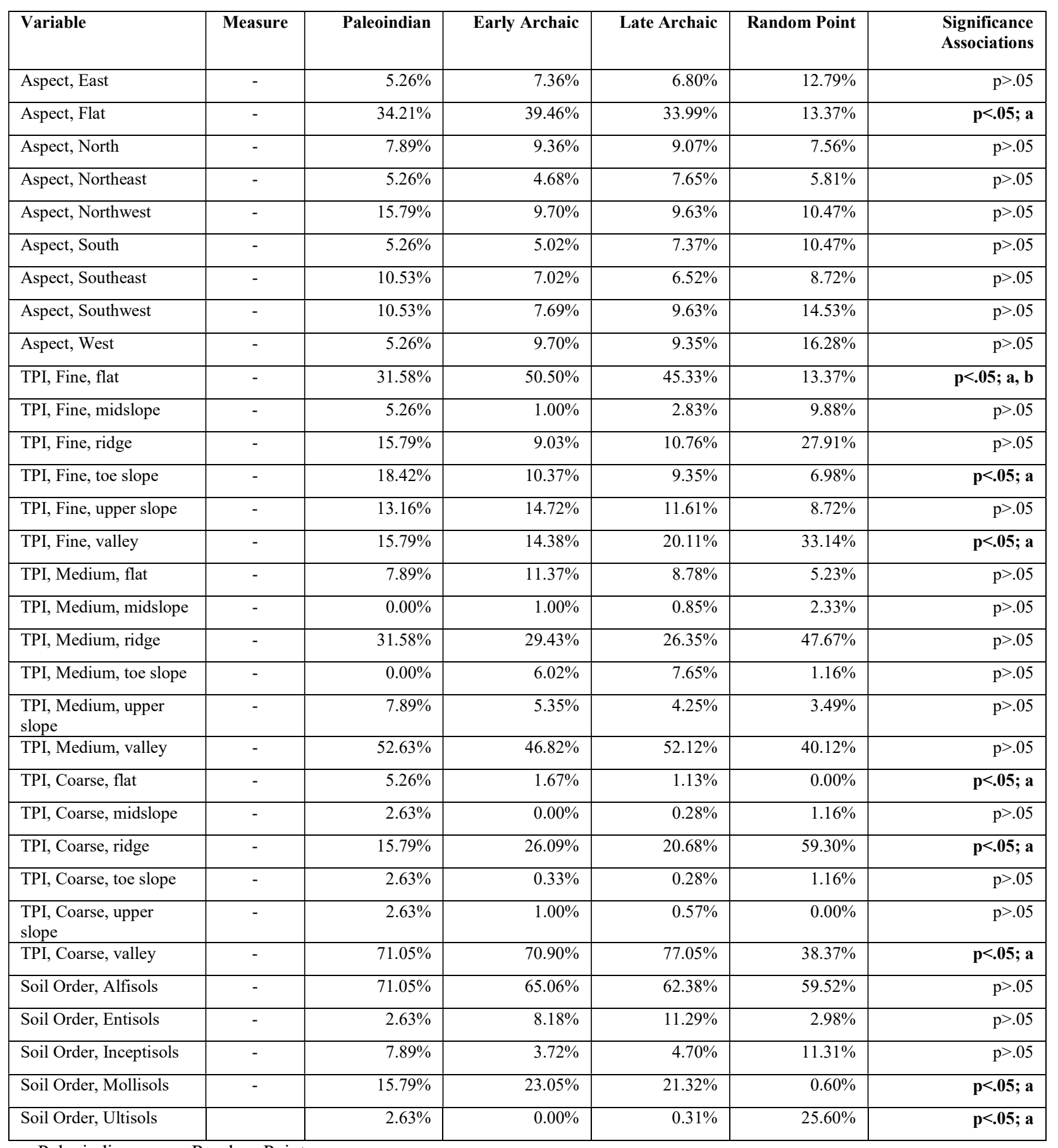

$\mathrm{a}=$ Paleoindian versus Random Point

$\mathrm{b}=$ Paleoindian versus Early Archaic

$\mathrm{c}=$ Paleoindian versus Late Archaic

$\mathrm{d}=$ Early Archaic versus Late Archaic 
Table S5. Interior Low Plateaus model values and statistical results

\begin{tabular}{|c|c|c|c|c|c|c|}
\hline Variable & Measure & Paleoindian & Early Archaic & Late Archaic & Random Point & $\begin{array}{l}\text { Significance } \\
\text { Associations }\end{array}$ \\
\hline \multirow{2}{*}{$\begin{array}{l}\text { Distance to Large River } \\
\text { Confluence Pt. }(\mathrm{km})\end{array}$} & $\bar{x}$ & 33.30 & 39.61 & 39.05 & 46.76 & $\mathrm{p}<.05 ; \mathrm{a}$ \\
\hline & $s$ & 14.50 & 10.38 & 11.04 & 15.95 & - \\
\hline \multirow{2}{*}{$\begin{array}{l}\text { Distance to Large- } \\
\text { Medium River } \\
\text { Confluence Pt. }(\mathrm{km})\end{array}$} & $\bar{x}$ & 23.77 & 24.23 & 25.60 & 20.29 & $\mathrm{p}>.05$ \\
\hline & $S$ & 1288 & 1331 & 1412 & 949 & - \\
\hline \multirow{3}{*}{$\begin{array}{l}\text { Distance to Large River } \\
(\mathrm{km})\end{array}$} & & 4.54 & 5.13 & 5.42 & 7.87 & $\mathrm{p}<\mathbf{0 5} ; \mathrm{a}$ \\
\hline & $x$ & & & & & pon, \\
\hline & $s$ & 7.38 & 7.71 & 8.48 & 5.94 & - \\
\hline \multirow{2}{*}{$\begin{array}{l}\text { Distance to Medium } \\
\text { River }(\mathrm{km})\end{array}$} & $\bar{x}$ & 12.71 & 12.74 & 12.96 & 10.86 & $\mathrm{p}>.05$ \\
\hline & $s$ & 10.75 & 9.05 & 10.14 & 6.37 & - \\
\hline \multirow{2}{*}{$\begin{array}{l}\text { Distance to Wetland } \\
(\mathrm{km})\end{array}$} & $\bar{x}$ & 0.22 & 0.29 & 0.26 & 1.01 & $\mathbf{p}<.05$; a \\
\hline & $s$ & 0.15 & 0.27 & 0.27 & 0.63 & - \\
\hline \multirow[t]{2}{*}{ Distance to Trail $(\mathrm{km})$} & $\bar{x}$ & 1.07 & 2.04 & 1.35 & 5.13 & $\mathbf{p}<.05 ;$ a \\
\hline & $s$ & 1.23 & 3.11 & 1.90 & 3.45 & - \\
\hline \multirow{2}{*}{$\begin{array}{l}\text { Distance to Sand Dune } \\
(\mathrm{km})\end{array}$} & $\bar{x}$ & 61.74 & 49.28 & 51.36 & 22.28 & $\mathbf{p}<.05 ;$ a \\
\hline & $s$ & 36.16 & 29.40 & 30.77 & 9.82 & - \\
\hline \multirow[t]{2}{*}{ TRI, Fine Scale } & $\bar{x}$ & 61.5 & 54.5 & 64.2 & 132.5 & $\mathbf{p}<.05 ;$ a \\
\hline & $s$ & 37.2 & 28.0 & 39.0 & 37.8 & - \\
\hline \multirow[t]{2}{*}{ TRI, Medium Scale } & $\bar{x}$ & 158.2 & 160.6 & 161.1 & 211.1 & $\mathbf{p}<.05 ;$ a \\
\hline & $s$ & 38.0 & 54.3 & 57.1 & 35.9 & - \\
\hline \multirow[t]{2}{*}{ TRI, Coarse Scale } & $\bar{x}$ & 323.4 & 332.0 & 332.7 & 280.6 & $\mathrm{p}>.05$ \\
\hline & $s$ & 120.7 & 115.9 & 114.5 & 29.4 & - \\
\hline \multirow{2}{*}{$\begin{array}{l}\text { Landform Curvature, } \\
\text { Fine }\end{array}$} & $\bar{x}$ & 4.3 & 0.5 & -0.5 & -5.9 & $\mathrm{p}>.05$ \\
\hline & $s$ & 14.7 & 10.3 & 13.0 & 36.0 & - \\
\hline \multirow{2}{*}{$\begin{array}{l}\text { Landform Curvature, } \\
\text { Medium }\end{array}$} & $\bar{x}$ & -10.0 & -23.1 & -12.7 & -5.3 & $\mathrm{p}>.05$ \\
\hline & $s$ & 36.3 & 66.0 & 64.2 & 78.6 & - \\
\hline \multirow{2}{*}{$\begin{array}{l}\text { Landform Curvature, } \\
\text { Coarse }\end{array}$} & $\bar{x}$ & -50.4 & -54.9 & -40.3 & 1.4 & $\mathbf{p}<.05 ; \mathbf{a}$ \\
\hline & $s$ & 62.7 & 87.4 & 87.7 & 77.8 & - \\
\hline \multirow[t]{2}{*}{ Elevation (m) } & $\bar{x}$ & 174.8 & 181.1 & 183.0 & 234.8 & $\mathbf{p}<.05 ; \mathbf{a}$ \\
\hline & $s$ & 33.7 & 44.1 & 42.4 & 41.2 & - \\
\hline \multirow{2}{*}{$\begin{array}{l}\text { Distance to Karst } \\
\text { Landscape }(\mathrm{km})\end{array}$} & $\bar{x}$ & 1.51 & 2.30 & 1.92 & 63.43 & $\mathbf{p}<.05 ; \mathbf{a}$ \\
\hline & $s$ & 2.68 & 3.38 & 3.04 & 25.90 & - \\
\hline \multirow[t]{2}{*}{ Slope, Fine } & $\bar{x}$ & 3.7 & 3.3 & 3.3 & 8.3 & $\mathbf{p}<.05 ; \mathbf{a}$ \\
\hline & $s$ & 3.0 & 3.1 & 2.9 & 6.0 & - \\
\hline \multirow[t]{2}{*}{ Slope, Medium } & $\bar{x}$ & 2.1 & 2.2 & 2.1 & 1.5 & $\mathrm{p}>.05$ \\
\hline & $s$ & 1.4 & 1.4 & 1.3 & 1.0 & - \\
\hline \multirow[t]{2}{*}{ Slope, Coarse } & $\bar{x}$ & 0.1 & 0.1 & 0.1 & 0.1 & $\mathrm{p}>.05$ \\
\hline & $s$ & 0.1 & 0.1 & 0.1 & 0.0 & - \\
\hline \multirow[t]{2}{*}{ Viewshed (km2) } & $\bar{x}$ & 144.7 & 242.6 & 182.6 & 365.6 & $\mathbf{p}<.05 ;$ a \\
\hline & $s$ & 314.1 & 665.0 & 472.0 & 1116.9 & - \\
\hline
\end{tabular}




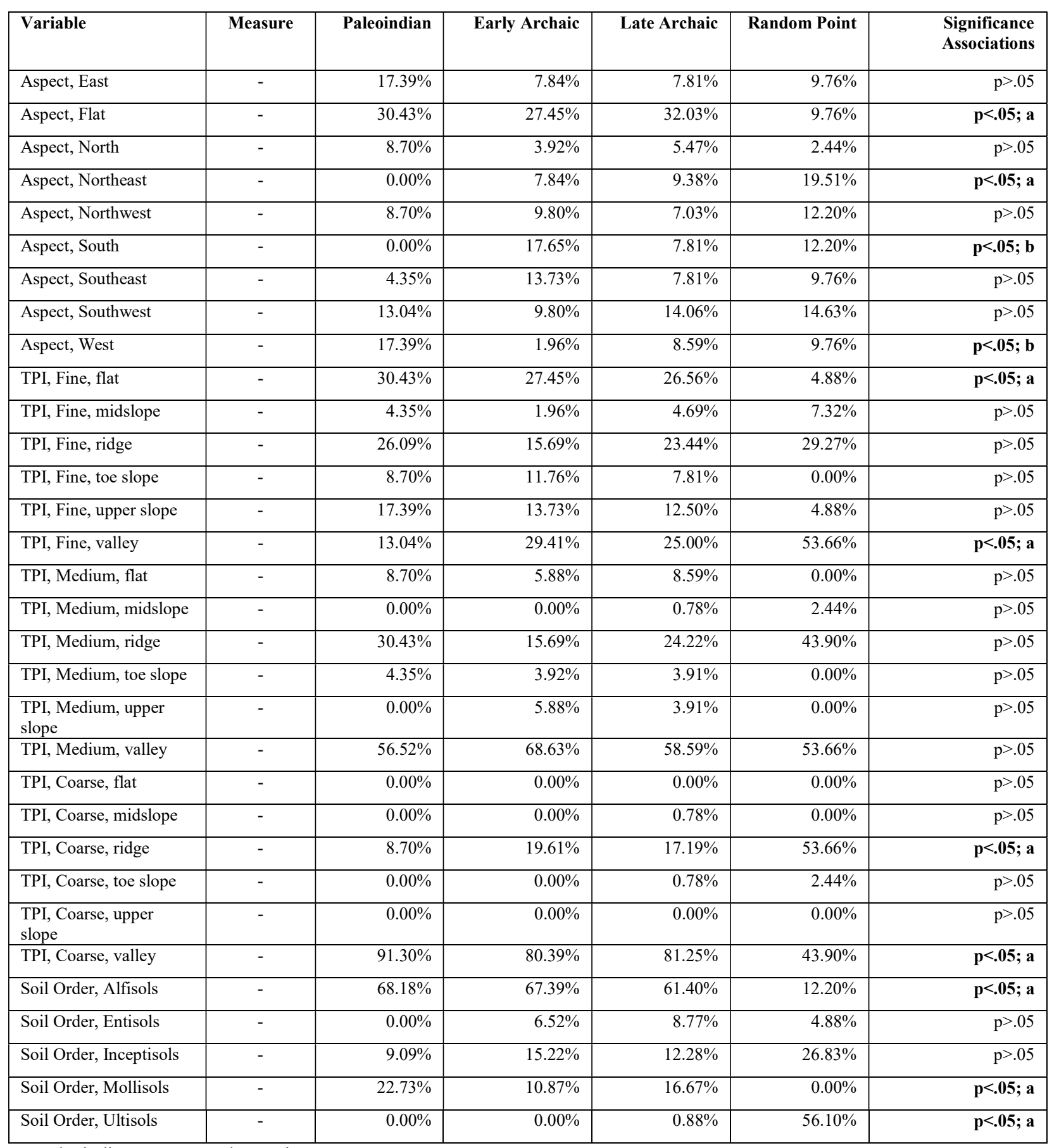

$\mathrm{a}=$ Paleoindian versus Random Point

$\mathrm{b}=$ Paleoindian versus Early Archaic

$\mathrm{c}=$ Paleoindian versus Late Archaic

$\mathrm{d}=$ Early Archaic versus Late Archaic 


\section{S1.3 Data results for the Binary Logistic Regression test}

Binary logistical regression is similar to linear regression except that "instead of predicting the value of a variable $\mathrm{Y}$ from a predicator variable $\mathrm{X} 1$, or several predictor variables $(\mathrm{Xs})$, we predict the probability of Y occurring given known values of X1 (or Xs)" (Field, 2009: 266). The equation for logistical regression with multiple predictor variables is:

$$
\begin{aligned}
& \mathrm{P}(\mathrm{Y})= 1 / 1+\mathrm{e}^{\wedge}-(\mathrm{b} 0+\mathrm{b} 1 \mathrm{X} 1 \mathrm{i},+\mathrm{b} 2 \mathrm{X} 2 \mathrm{i} \ldots \mathrm{BnXni}) \\
& \cdot \mathrm{P}=\text { Probability } \\
& \cdot \mathrm{Y}=\text { Outcome variable (dependent variable) } \\
& \quad \mathrm{e}=\text { natural log }(2.71) \\
& \mathrm{b} 0=\mathrm{Y} \text { intercept (or constant) } \\
& \mathrm{b} 1=\text { gradient } \\
& \mathrm{X} 1=\text { value of the independent (or predictor) variable }
\end{aligned}
$$

A series of chi-square $\left(\chi^{2}\right)$ (categorical variables) and Mann-Whitney $(U)$ (continuous variables) statistical tests were run between the 54 variables calculated for Paleoindian site locations and random point locations (see main text). Of these, 31 variable associations were significant for the Comprehensive Model and were further tested through binary logistic regression in SPSS. The binary logistical regression analysis was used to test the proportional strength of significant results to determine if certain variables were more influential than others in determining site location. Although theoretically, additional binary logistic regression tests could be calculated on

\begin{tabular}{|c|c|c|c|c|c|c|c|c|c|}
\hline & \multirow[t]{2}{*}{ B } & \multirow[t]{2}{*}{ S.E. } & \multirow[t]{2}{*}{ Wald } & \multirow[t]{2}{*}{ df } & \multirow[t]{2}{*}{ Sig. } & \multirow[t]{2}{*}{$\operatorname{Exp}(B)$} & \multicolumn{2}{|c|}{ 95\% C.I.for EXP(B) } \\
\hline & & & & & & & & Lower & Upper \\
\hline \multirow{7}{*}{$\begin{array}{l}\text { Step } \\
1\end{array}$} & $\begin{array}{l}\text { Province, Interior Low } \\
\text { Plateaus }\end{array}$ & 2.638 & .630 & 17.522 & 1 & .000 & 13.989 & 4.067 & 48.114 \\
\hline & Soil Order, Mollisols & 2.305 & .785 & 8.618 & 1 & .003 & 10.026 & 2.152 & 46.722 \\
\hline & Province, Central Lowland & 1.388 & .366 & 14.370 & 1 & .000 & 4.008 & 1.955 & 8.216 \\
\hline & Soil Order, Ultisols & -1.203 & .754 & 2.546 & 1 & .111 & .300 & .068 & 1.316 \\
\hline & Soil Order, Alfisols & 1.160 & .447 & 6.733 & 1 & .009 & 3.190 & 1.328 & 7.663 \\
\hline & $\begin{array}{l}\text { Distance to Wetland } \\
(\mathrm{km})\end{array}$ & -.001 & .000 & 9.938 & 1 & .002 & .999 & .998 & .999 \\
\hline & $\begin{array}{l}\text { Distance to Large River } \\
(\mathrm{km})\end{array}$ & .000 & .000 & .950 & 1 & .330 & 1.000 & 1.000 & 1.000 \\
\hline
\end{tabular}
subgroups in this sample (e.g., by physiographic province), the small Paleoindian sample size makes this approach undesirable.

Table S6. SPSS output model results

\begin{tabular}{|l|r|r|r|}
\hline Step & $\mathbf{- 2}$ Log likelihood & Cox \& Snell R Square & Nagelkerke R Square \\
\hline 1 & $301.802^{\mathrm{a}}$ & .313 & .593 \\
\hline
\end{tabular}

Table S7. SPSS independent variables in logistic regression equation showing coefficients, standard error, Wald statistic, and odds ratio $(\operatorname{Exp}[\mathrm{B}])$ and confidence intervals. 


\begin{tabular}{|c|c|c|c|c|c|c|c|c|}
\hline & \multirow[t]{2}{*}{$\mathbf{B}$} & \multirow[t]{2}{*}{ S.E. } & \multirow[t]{2}{*}{ Wald } & \multirow[t]{2}{*}{ df } & \multirow[t]{2}{*}{ Sig. } & \multirow[t]{2}{*}{$\operatorname{Exp}(B)$} & \multicolumn{2}{|c|}{ 95\% C.I.for EXP(B) } \\
\hline & & & & & & & Lower & Upper \\
\hline $\begin{array}{l}\text { Distance to Large River } \\
\text { Confluence Pt. }(\mathrm{km})\end{array}$ & .000 & .000 & .006 & 1 & .936 & 1.000 & 1.000 & 1.000 \\
\hline Distance to Trail (km) & .000 & .000 & 10.033 & 1 & .002 & 1.000 & 1.000 & 1.000 \\
\hline TRI, Fine Scale & -.015 & .007 & 4.584 & 1 & .032 & .985 & .972 & .999 \\
\hline TRI, Medium Scale & -.009 & .005 & 3.478 & 1 & .062 & .991 & .981 & 1.000 \\
\hline TRI, Coarse Scale & .007 & .004 & 2.925 & 1 & .087 & 1.007 & .999 & 1.015 \\
\hline $\begin{array}{l}\text { Landform Curvature, } \\
\text { Coarse }\end{array}$ & -.001 & .003 & .041 & 1 & .840 & .999 & .994 & 1.005 \\
\hline Elevation (m) & -.004 & .006 & .461 & 1 & .497 & .996 & .984 & 1.008 \\
\hline $\begin{array}{l}\text { Distance to Karst } \\
\text { Landscape }(\mathrm{km})\end{array}$ & .000 & .000 & .040 & 1 & .842 & 1.000 & 1.000 & 1.000 \\
\hline Slope, Fine & -.045 & .053 & .729 & 1 & .393 & .956 & .862 & 1.060 \\
\hline Slope, Medium & .198 & .174 & 1.297 & 1 & .255 & 1.219 & .867 & 1.714 \\
\hline Viewshed (km2) & .000 & .000 & .757 & 1 & .384 & 1.000 & 1.000 & 1.000 \\
\hline Aspect, Flat & -.664 & .407 & 2.657 & 1 & .103 & .515 & .232 & 1.144 \\
\hline Aspect, South & -.649 & .636 & 1.041 & 1 & .308 & .522 & .150 & 1.819 \\
\hline TPI, Fine, flat & .320 & .517 & .383 & 1 & .536 & 1.378 & .500 & 3.798 \\
\hline TPI, Fine, ridge & .454 & .543 & .698 & 1 & .403 & 1.575 & .543 & 4.569 \\
\hline TPI, Fine, toe slope & .466 & .599 & .606 & 1 & .436 & 1.594 & .493 & 5.159 \\
\hline TPI, Fine, valley & -.070 & .548 & .016 & 1 & .898 & .932 & .318 & 2.730 \\
\hline TPI, Medium, flat & .294 & .573 & .263 & 1 & .608 & 1.342 & .436 & 4.127 \\
\hline TPI, Medium, ridge & -.112 & .432 & .068 & 1 & .795 & .894 & .383 & 2.085 \\
\hline $\begin{array}{l}\text { TPI, Medium, upper } \\
\text { slope }\end{array}$ & -.011 & .694 & .000 & 1 & .988 & .989 & .254 & 3.855 \\
\hline TPI, Coarse, ridge & -1.932 & .735 & 6.898 & 1 & .009 & .145 & .034 & .613 \\
\hline TPI, Coarse, valley & -.447 & .719 & .387 & 1 & .534 & .640 & .156 & 2.615 \\
\hline Constant & .505 & 1.878 & .072 & 1 & .788 & 1.658 & & \\
\hline
\end{tabular}




\section{Chapter 3. Introduction and Summary of Findings}

A reanalysis of existing collections and results of new 2015-16 excavation data for Sandy Springs was the subject for the second dissertation chapter in Purtill (2015). Initial results include a reevaluation of Sandy Springs concerning its role in Paleoindian settlement systems and subsistence strategies. Project results were published in 2017 in the Journal of Archaeological Science, Reports (Purtill, 2017), the full text of which is included in Appendix A. The primary findings of this research are as follows:

- The previous claim of saline springs at the Paleoindian Sandy Springs site by Roger Cunningham is tested. Saline groundwater is seen as a vital draw for game animals and human hunters.

- Electrical conductivity and $\mathrm{pH}$ analysis is used to investigate the presence of saline groundwater but tests fail to confirm its presence at the site or within the region. Thus, the accuracy of a significant component of the proposed settlement and subsistence models for Sandy Springs is questioned.

- Alternative explanations are forwarded to explain Paleoindian occupancy at Sandy Springs including the presence of a rare xeric ecosystem and location along an early trail system connecting the Cumberland, Tennessee, and Ohio river valleys.

This research also resulted in additional site-specific supplemental information regarding artifact assemblages not included in the 2017 publication. Specifically, the 1976 and 2015-16 artifact assemblages are analyzed based on procedures outlined in Purtill (2015). It is anticipated that supplemental information will provide the basis for a future article that detail various aspects of the Sandy Springs site. Topics that supplemental information may address include better definition of the 1974 National Register boundaries for Sandy Springs, spatial distribution of artifacts and activity areas including those thought to represent Paleoindian occupation, and the potential that Paleoindian deposits are buried under modern sand dune landforms. 
Chapter 3. Manuscript of Published Journal Article

Status: published in 2017

\title{
Purtill, M. P. (2017). Reconsidering the Potential Role of Saline Springs in the Paleoindian Occupation of Sandy Springs, Adams County, Ohio. Journal of Archaeological Science: Reports, 13, 164-174. https://doi.org/10.1016/j.jasrep.2017.03.054
}

\begin{abstract}
:
The potential role of saline springs in Paleoindian settlement models has been espoused for over 50 years. An early example of this adaptive strategy was proposed in 1973 by Roger

Cunningham in his formative discussion of the Paleoindian Sandy Springs site in Adams County, Ohio. Cunningham argued that saline springs located among local sand dunes acted as a significant draw for migrating game animals and, subsequently, the Paleoindian hunters that pursued them. Despite being widely accepted and repeated in the literature, the claim of salineenriched groundwater at Sandy Springs has never been evaluated quantitatively. To assess the accuracy of Cunningham's claim, this study completed electrical conductivity and $\mathrm{pH}$ analysis of surface water and sediment samples within a 20-km radius of Sandy Springs. Testing failed to identify water samples $>320 \mathrm{ppm}$ total dissolved solids, a result far below established thresholds for brackish or brine classification. Underlying local bedrock geology also is not conducive for the presence of saline springs, and no mention of salt licks, commercial salt wells, or animal trails has been identified in the literature for Adams County, Ohio. Sediment samples from sand dunes locations previously argued to contain saline springs at Sandy Springs also failed to yield statistically different $\mathrm{pH}$ values than those from non-dune, alluvial contexts. Overall, the reported presence of saline springs at Sandy Springs could not be verified by geochemical data. Instead, it is suggested in this article that saline groundwater was not a principal factor in determining Paleoindian occupation of the site. Alternative explanations for site occupancy include the possible presence of rare resources associated with a sand prairie ecosystem and the potential that Sandy Springs was positioned along an early trail system connecting the Upper Ohio Valley with the Tennessee and Cumberland Valleys.
\end{abstract}

Keywords:

Electrical conductivity

Sandy Springs

Paleoindian

Saline Springs

Upper Ohio River Valley 


\section{Introduction}

In the inaugural 1973 issue of Archaeology of Eastern North America, Roger Cunningham was among the earliest to suggest that the location of natural saline springs was a significant draw for late Pleistocene herbivores and the Paleoindian hunters who pursued them in the Upper Ohio Valley (Cunningham, 1973, p. 121; see also Rolingson, 1964, p. 72). Cunningham referred to the proximity of Paleoindian artifacts, saline springs, and the remains of Pleistocene megafauna such as mammoth (Mammut americanus) and bison (Bison antiquus) at northern Kentucky sites such as Big Bone Lick and Upper and Lower Blue Licks (M. C. Hansen, 1983, 1995; Rolingson, 1964) as evidence for this adaptive land-use strategy. As additional support for this pattern, Cunningham further discussed several newly discovered Upper Ohio Valley sites that he claimed yielded Paleoindian material in association with saline springs (Cunningham, 1973, pp. 120122). Since 1973, a purported correlation between saline waters and Paleoindian sites in the eastern U.S. has become widely accepted and embedded in the literature (e.g., Anderson \& Gillam, 2000, p. 44; Brown, 1999, p. 115, 2010; Dincauze, 1993; Maggard \& Stackelbeck, 2008, p. 154; O’Brien, 1996, pp. 446-451; Smith, 1990, pp. 98-111, 1990, p. 244, Kenneth B. Tankersley, 1996, p. 28, 1998, p. 14).

Most prominent among these "new" sites discussed by Cunningham was Sandy Springs in southeastern Adams County, Ohio (Figure 3-1). Based on amateur collector data, Cunningham characterized Sandy Springs as a dense concentration of Paleoindian material distributed among relict sand dunes and saline springs (Cunningham, 1973, p. 122). In 1994, Mark Seeman and colleagues expanded upon Cunningham's description by offering various hypotheses concerning site age and function based on their own analysis of local artifact collections. Notable was the confirmation of at least 98 Paleoindian points from the site (Seeman et al., 1994, p. 81). Repeated occupation throughout the Paleoindian period is suggested by an inventory of Clovis ${ }^{1}$ $(n=14)$, Cumberland $(n=15)$, and lanceolate $(n=34)$ point styles. A high frequency of intact points implied to Seeman et al. (1994, p. 81) that Sandy Springs was neither a primary tool production area nor multi-activity base camp, but instead reflected a series of temporary hunting-collecting stations.

In Seeman et al.'s 1994 article, Cunningham's earlier claim of saline water at Sandy Springs was repeated and a personal communication was cited suggesting that springs recharge "from clay seams among or immediately below the dunes" (Seeman et al., 1994, p. 79). In the same passage, Seeman et al. refers to an early nineteenth century salt works across the Ohio River in Vanceburg, Kentucky, as further support of the claim of high water salinity in area streams. Finally, they cite historical reports of a natural ford on the Ohio River, just upriver from Sandy Springs, as a favorable crossing point for both humans and migrating game (Seeman et al., 1994, p. 79; see also Seeman \& Prufer, 1982, p. 157).

\footnotetext{
${ }^{1}$ In the original publication, Seeman et al., (1994, p. 81) partitioned “Clovis" point taxon into Clovis and Gainey styles. Recent studies, however, have questioned the utility of the "Gainey" taxon suggesting it is not based on empirical or quantitative evidence (e.g., Eren \& Desjardine, 2015, pp. 109-110; Eren, Vanderlaan, \& Holland, 2011). For this study, the Clovis $(n=2)$ and Gainey $(n=12)$ inventory was combined and labeled as Clovis.
} 


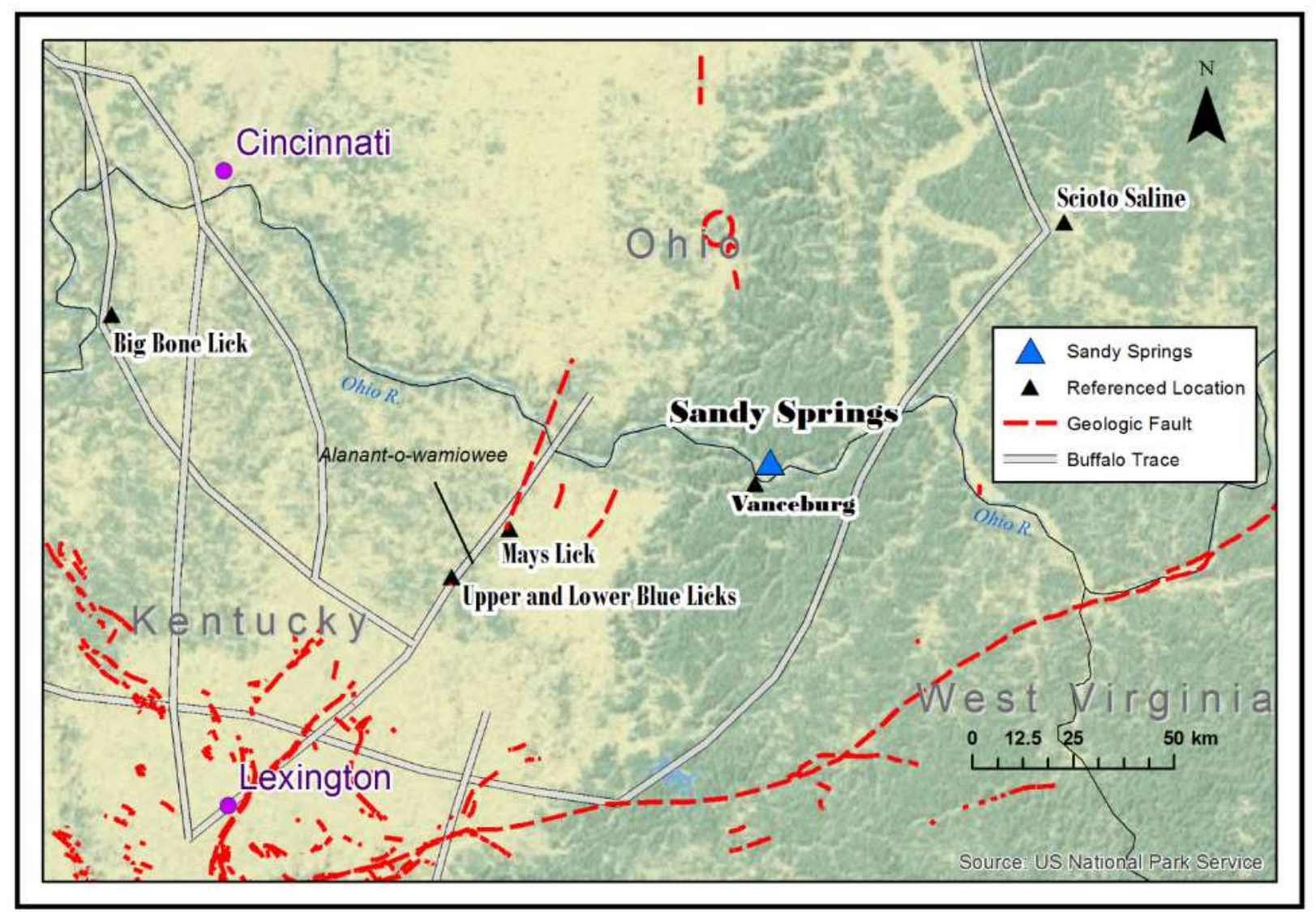

Figure 3-1. Geographic location of Sandy Springs Paleoindian site and locations referenced in text. Geologic fault locations based on Baranoski, 2013, and the Kentucky Geological Survey, 2016. Please note that a small geologic fault exists at the location of the Upper and Lower Blue Licks symbol. Buffalo trace locations based on Jakle, 1969, p. 690.

The report of saline springs has played a significant role in Cunningham's, and later Seeman et al.'s, interpretation as to why Sandy Springs was occupied repeatedly during the Paleoindian period. The presence of saline waters is thought to have attracted migrating game, especially herbivores, which could easily ford the Ohio River near Sandy Springs. Along the hummocky dune surface, it was suggested that Paleoindian hunters found excellent vistas of the surrounding landscape for game monitoring and topographic depressions in which hunters could seek concealment (Cunningham, 1973, pp. 120-122; Seeman et al., 1994, pp. 83-84). This elegant narrative, based largely on the supposition of saline springs, has important implications for many extant eastern U.S. Paleoindian adaptive land-use models, prey choice strategies, and mobility patterns (e.g., Anderson, 2013; Broster et al., 2013; Cannon \& Meltzer, 2004, 2008; Kelly \& Todd, 1988; Lepper \& Meltzer, 1991; Meltzer \& Smith, 1986; Smallwood, 2012; Surovell \& Waguespack, 2009; Kenneth B. Tankersley, 1990; Waguespack \& Surovell, 2003). For example, this perspective posits Paleoindians at Sandy Springs as 'specialized hunters' (e.g., Kelly \& Todd, 1988, p. 240; Kenneth B. Tankersley et al., 1990) reducing risk by revisiting a landscape characterized by predictable, and abundant, animal resources. This incorporates components of both 'place-oriented' and 'technology oriented' strategies as the repeated reoccupation of Sandy Springs (place-oriented) is thought to have supported a specialized hunting lifestyle (technology- 
oriented). A similar argument has been made for the Paleoindian occupation of Sheridan Cave, Huron County, Ohio, where Redmond and Tankersley (2005, p. 524) suggested periodic revisits to the sinkhole/cave to scavenge, or killed, entrapped animals.

The claim of saline waters at Sandy Springs largely has been accepted in the Paleoindian literature (e.g., Gramly \& Funk, 1990, p. 16; Lepper, 1986, p. 53; Lothrop \& Cremeens, 2010, p. 121; Smith, 1990, p. 244; Tune, 2016, p. 311), despite the fact that ionic concentrations of local water sources have not been directly measured through gravimetric or conductivity means. The origin of Cunningham's initial claim is unclear but he cites an extended passage from Stout et al. (1932, p. 11) on the importance and location of Ohio brines and highlights the fact that several local stream names are suggestive of hypersalinity such as Sulfur Creek and Long Lick Creek in Ohio and Salt Lick Creek in Kentucky (Cunningham, 1973, p. 122). Close review of the Stout et al., passage, however, suggests that the authors only reference salt sources in eastern, not western, Ohio. Moreover, nowhere in their volume do the authors list Adams County, Ohio, as containing saline springs or commercial salt wells.

Given this ambiguity, the purpose of this research was to evaluate the long-held assumption that the Sandy Springs landscape contains natural saline waters of such concentration as to have attracted species pursued by late Pleistocene hunters. This study included review of relevant geological literature on potential saline groundwater sources and identification of historical passages that mention regional brackish waters or salt production, including information regarding the salt works noted at Vanceburg, Kentucky. Finally, electrical conductivity testing of surface water and sediment, and $\mathrm{pH}$ testing of sediment from dune and non-dune contexts, were used to assess the salinity of surface waters in the general Sandy Springs area. Electrical conductivity of surface waters from two renowned Ohio Valley saline springs, Big Bone Lick in Union, Kentucky (Kenneth B. Tankersley, 1985, 1996, 2007; Kenneth B. Tankersley, Waters, \& Stafford Jr, 2009), and the Scioto Saline in Jackson, Ohio (M. C. Hansen, 1983, 1995; Hildreth, 1838, p. 57; Y. Liu, Andersen, Williams, \& Jackson, 2013) (Figure 3-1), were tested and compared to Sandy Springs results.

\section{Distribution and composition of saline springs in the eastern U.S.}

"Springs, in the form of seeps and rills and often saline, appear regularly among these high sand terraces and dunes and appear to have been important attractions for herbivores and thus for man who preyed upon them."

Roger Cunningham, 1973, pp. 120-121 (italics added)

Although Cunningham is correct in stating that fresh water springs are common, saline springs are rare in the eastern U.S. due to the humid climate and solubility of exposed evaporite beds (Phalen, 1919, p. 14). The composition, concentration, and location of soluble salts in groundwater vary according to climate, bedrock geology, hydraulic conductivity and gradient, preferential flow paths, and ultimate saline source (Hillel, 2013, pp. 233-257; Todd \& Mays, 2005, pp. 329-333). When present in the eastern U.S., such springs typically result from dissolution of deep-seated evaporitic rocks rich in anhydrite $\left(\mathrm{CaSO}_{4}\right)$, gypsum $\left(\mathrm{CaSO}_{4} * 2 \mathrm{H}_{2} \mathrm{O}\right)$, and halite $(\mathrm{NaCl})$ minerals (Carlson, 1991, p. 11). They are often associated with sedimentary environments where dense fluids and hydrocarbons tend to collect. A second potential saline 
source is connate waters trapped in deep, porous bedrock sections such as sandstones under high hydrostatic pressures and overlain by faulted bedrock. In such contexts, brines and saline solutions may discharge to the surface where they are subject to plant uptake, evaporation, and mineral precipitation as compounds such as gypsum $\left(\mathrm{CaSO}_{4} * 2 \mathrm{H}_{2} \mathrm{O}\right)$.

In some cases, saline water is enriched in sulfur concentrations, often marked by a bluish-white color and sulfur odor (e.g., $\mathrm{H}_{2} \mathrm{~S}$ ). Local bacterial reduction of sulfates (e.g., $\mathrm{SO}_{4}$ ) appears to characterize some springs (McCartney, Finney, \& Maynard, 2005; see also Hem, 1985, pp. 116117). Available water chemistry data on Ohio Valley brines derive from deep well samples where salinity is more concentrated due to variable-density flow paths and interaction between bedrock and fresh groundwater. Typical geochemical gradients include near-surface groundwater rich in bicarbonate anions with chloride-enriched waters occupying deeper sections (Todd \& Mays, 2005, p. 330). Stout et al. (1932, pp. 15-18) report that deep well samples (>500 m) in eastern Ohio produce heavily concentrated brines containing values in the 170,000 to 240,000 ppm total dissolved solids (TDS) range.

When discharged at the surface, saline springs were colloquially referred to as salt licks, or mineral licks, by early Euro-American settlers (M. C. Hansen, 1995, p. 2; Jakle, 1969; Stout , Lamborn, Raymond E., Schaaf, Downs., 1932, p. 15) due to the predilection of ungulates to "lick the earth on account of the saline particles with which it is impregnated" (Imlay, 1793, p. 43). Consumption of salt, especially sodium-based compounds, is essential for various physiological functions in mammals from maintaining osmotic balance in body fluids to facilitating electrical conductivity between nerves (Dethier, 1977, p. 744). The need for supplementary sodium, often on a seasonal basis, is especially prevalent in herbivores of eastern North America where foraged diets typically are sodium-poor (Botkin, Jordan, Dominski, Lowendorf, \& Hutchinson, 1973; D. Fraser \& Reardon, 1980, p. 36; Hellgren \& Pitts, 1997; Weeks \& Kirkpatrick, 1976). Various strategies have been adapted by animals to supplement low-sodium diets such as the geophagous consumption of sodium-enriched soils found through seasonal migrations to saline springs or seeps (e.g., Kreulen, 1985).

Numerous licks such as Big Bone Lick and Upper and Lower Blue Licks of northern Kentucky, Saltville in southwestern Virginia, and the Scioto Saline in eastern Ohio, appear to have been producing saline waters since at least the late Pleistocene based on the presence of fauna of extinct species including mastodon (Mammut americanus), muskox (Bootherium bombifrons), bison (Bison antiquus), and stag-moose (Cervalces scotti), among others (R.A. Boisvert, 1984; M. C. Hansen, 1995, p. 1; C. E. Ray, Cooper, \& Benninghoff, 1967; Kenneth B. Tankersley et al., 2009). In the historic period, licks are known to have attracted seasonally migrating bison (Bison bison americanus) which resulted in the development of well-defined trails called buffalo traces (Jakle, 1969, pp. 691-692; Wilcox, 1970) (see Figure 3-1).

\section{Sandy Springs Environmental Context}

Sandy Springs is located on a broad, $2.4 \mathrm{~km}$ wide meanderbelt of the Ohio River (Figure 3-2). Situated in the heavily dissected Shawnee-Mississippian Plateau of the Appalachian Plateaus province (Brockman, 2006), Sandy Springs is underlain by Silurian, Devonian, and Mississippian shales, limestones, siltstones, and thickly bedded sandstones. Three geomorphic 
surfaces (S1-S3) rise above a modern floodplain (S0). The S1 surface displays a pronounced ridge-and-swale topography and is interpreted as a Holocene alluvial landform correlative to those at Stuart Station, only 40 km downstream of Sandy Springs (Purtill, 2012, pp. 21-41). S2 and S3 surfaces have been interpreted as late Wisconsin outwash terraces (Morris \& Pierce, 1967; Pavey et al., 1999) and reportedly contain up to $45 \mathrm{~m}$ of well-sorted gravels, sands, silts, and clay.

The upper surfaces on both the Ohio and Kentucky sides of the river contain a $~ 304$ ha dune field characterized by prominent relict sand dunes up to $18 \mathrm{~m}$ in height (Morris \& Pierce, 1967; Purtill, 2016; Purtill \& Kite, 2015). Springs have been reported to exist among these dunes (Cunningham, 1973, pp. 121-122) and the author observed one active seep emerging from a sand sheet near U.S. 52. At Sandy Springs, dunes are droughty and support remnant sand prairie vegetation which may be fed by natural springs. The prairie, which likely was more extensive in range prior to agricultural disturbance, supports many xeric plant species including eastern prickly pear cactus (Opuntia humifusa), passion flower (Passiflora incarnata), spreading sandwort (Arenaria patula), little whitlow grass (Draba brachycarpa) and silkgrass (Chrysopis graminifolia) (Noelle \& Blackwell, 1972; M. A. Vincent, Gardner, \& Riley, 2011).

\section{Materials and methods}

\subsection{Sample Locations}

A total of 16 surface water and 14 sediment samples were tested to characterize the potential for saline springs at Sandy Springs (Table 3-1; Figures 3-2 and 3-3). All but three (WS5, WS14, and WS15) derive from within $20 \mathrm{~km}$ of Sandy Springs. Water sample sources include McCall Run, Gilpen Run, Salt Lick Creek, Sulphur Creek, Upper Twin Creek, Turkey Creek, and an unnamed tributary. The active seep near U.S. 52 was sampled (WS10) and sediment samples were collected from a dry gully north of the Sandy Springs Cemetery where a second intermittent seep is reported to exist by local resident Carmel Taylor (141, 142, and 144). Sulphur Creek, a bedrock channel reach just west of Sandy Springs, was sampled at three locations (WS1, WS12, and WS13), each underlain by a different bedrock geology (Estill Shale, Pebbles Dolomite, and Ohio Shale, respectively), to determine if conductivity is constrained by lithology.

Table 3-1. Geographic location of sample sites.

\begin{tabular}{|l|l|l|r|r|}
\hline Sample Site and Context & Code & Sample Type & Latitude & Longitude \\
\hline Unnamed tributary next to dunes & WS11 & Surface Water & 38.61516 & -83.29716 \\
\hline Sand sheet seep & WS10 & Surface Water & 38.61332 & -83.30007 \\
\hline McCall Run & WS4 & Surface Water & 38.60905 & -83.28635 \\
\hline Ohio River & WS9 & Surface Water & 38.60555 & -83.31338 \\
\hline Gilpen Run Creek & WS8 & Surface Water & 38.62823 & -83.30711 \\
\hline Salt Lick Creek & WS7 & Surface Water & 38.60262 & -83.32183 \\
\hline Sulphur Creek & WS1 & Surface Water & 38.63729 & -83.32289 \\
\hline Sulphur Creek & WS13 & Surface Water & 38.64218 & -83.32178 \\
\hline Ohio River & WS3 & Surface Water & 38.64081 & -83.33139 \\
\hline
\end{tabular}




\begin{tabular}{|l|l|l|r|r|}
\hline Sample Site and Context & Code & Sample Type & Latitude & Longitude \\
\hline Salt Lick Creek & WS6 & Surface Water & 38.60111 & -83.34678 \\
\hline Upper Twin Creek & WS16 & Surface Water & 38.63331 & -83.25152 \\
\hline Sulphur Creek & WS12 & Surface Water & 38.65218 & -83.32134 \\
\hline Turkey Creek & WS2 & Surface Water & 38.69816 & -83.10456 \\
\hline Little Salt Creek & WS5 & Surface Water & 39.04706 & -82.63213 \\
\hline Salt spring, Big Bone Lick State Park & WS14 & Surface Water & 38.88643 & -84.75220 \\
\hline Big Bone Creek, Big Bone Lick State Park & WS15 & Surface Water & 38.88675 & -84.75250 \\
\hline Dune & $47,52,60,62$ & Sediment & 38.61378 & -83.29796 \\
\hline Sand Sheet & 4 & Sediment & 38.61294 & -83.29908 \\
\hline Sand sheet & 15 & Sediment & 38.61366 & -83.29931 \\
\hline Dune & 85 & Sediment & 38.61419 & -83.30052 \\
\hline Dune/Suspected Seep area & $141,142,144$ & Sediment & 38.61381 & -83.29768 \\
\hline Tributary Alluvium & 36,37 & Sediment & 38.61561 & -83.30053 \\
\hline Tributary Alluvium & 42,46 & Sediment & 38.61550 & -83.30088 \\
\hline
\end{tabular}

Seven sediment samples from sand dunes and a sand sheet were tested $(4,15,47,52,60,62$, and 85 ) to evaluate Cunningham's claim of saline springs being recharged from clay seams within, or immediately below, dunes (as cited in Seeman et al., 1994, p. 79). All dune samples are from soils classified as Plainfield Series (Lucht \& Brown, 1994) (Table 3-2). Four additional sediment samples $(36,37,42$, and 46), classified as Sciotoville Series soils, were collected from a finegrained alluvium of an unnamed tributary immediate west of the dunes to determine the potential that saline seeps emerge from below dune settings. To determine if $\mathrm{pH}$ values of dune sediments vary significantly from non-dune contexts, an additional 78 fine-grained sediment samples from alluvial contexts were tested. Alluvial samples were collected across the Sandy Springs meanderbelt area as part of a related geomorphological project currently being conducted by the author.

Finally, three non-Sandy Springs surface water samples were collected for comparative purposes. These samples include Big Bone Creek (WS15) in Union, Kentucky, an outlet stream of an active saline spring (WS14) adjacent to Big Bone Creek, and Little Salt Creek (Scioto Saline) in Jackson, Ohio (WS5). As mentioned previously, these locations are known to have been the focus of historical salt works and the presence of elevated water salinity is well established at each location. 


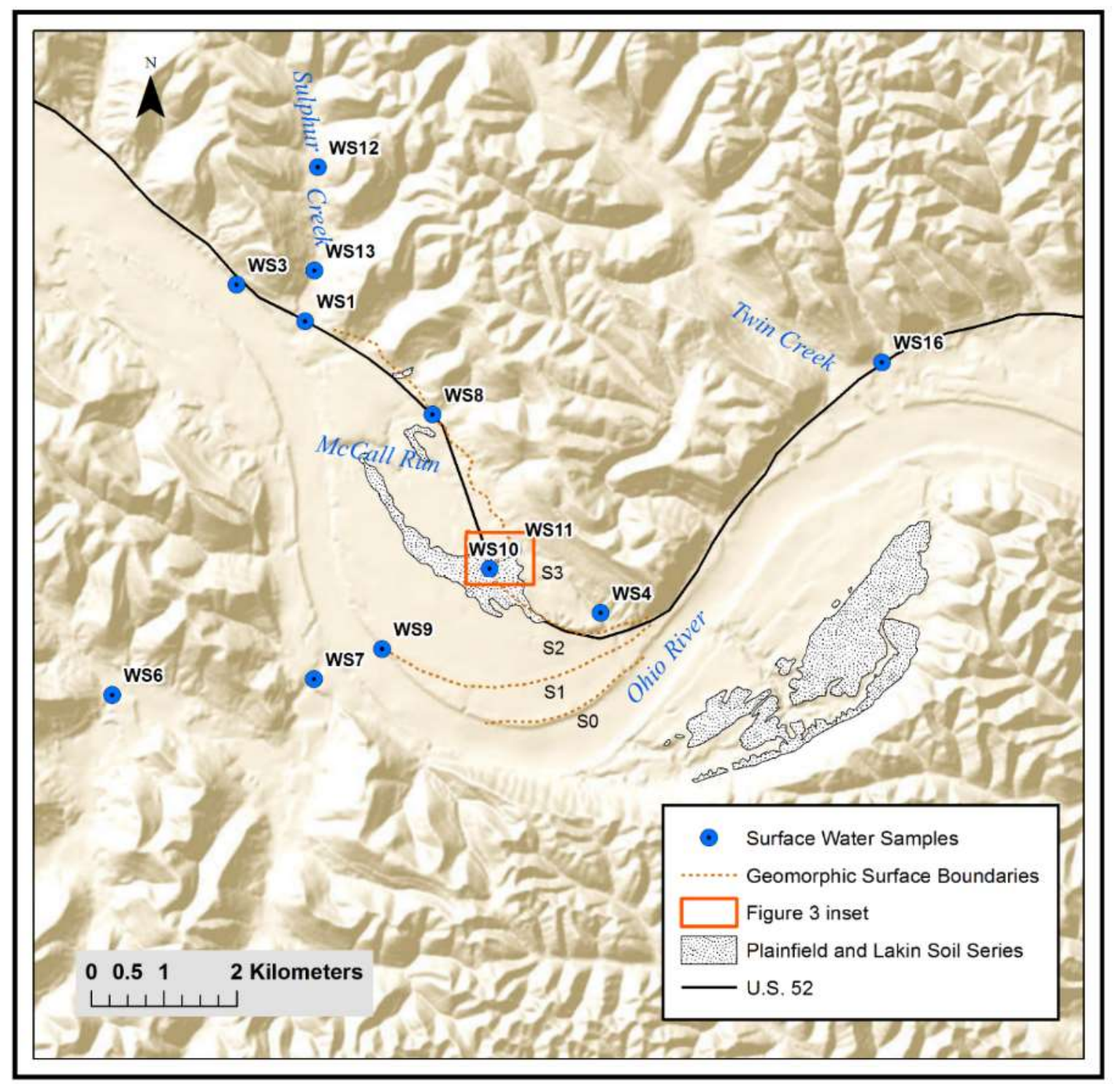

Figure 3-2. Location of surface water samples within the immediate Sandy Springs area. For convenience, Turkey Creek (WS2), located $19.4 \mathrm{~km}$ to the east, is not illustrated. Plainfield and Lakin Series symbol maps the distribution of sandy soils and dunes.

\subsection{Salinity}

Electrical conductivity was measured for all water and sediment samples to determine dissolved ionic concentration, or simply salinity (Walton, 1989). A Hanna Instruments HI9033 multi-range conductivity meter was used to measure water samples and a Mettler Toledo $\mathrm{pH}$ and conductivity meter was used for sediment samples. Water samples were measured directly whereas sediment samples were measured from a saturated paste consisting of 2:1 deionized water to sediment ( $5 \mathrm{~g}$ of sediment: $10 \mathrm{ml}$ of deionized water) mixture. Pastes were agitated and allowed to stand overnight before measurement. Salinity for sediment and water samples initially were calculated as microSiemens $(\mu \mathrm{S} / \mathrm{cm})$ compensated to $25^{\circ} \mathrm{C}$. To facilitate inter-study 
comparability, $\mu \mathrm{S} / \mathrm{cm}$ units were divided by a standard correction factor of 1.56 to convert to estimated ppm TDS (Walton, 1989).

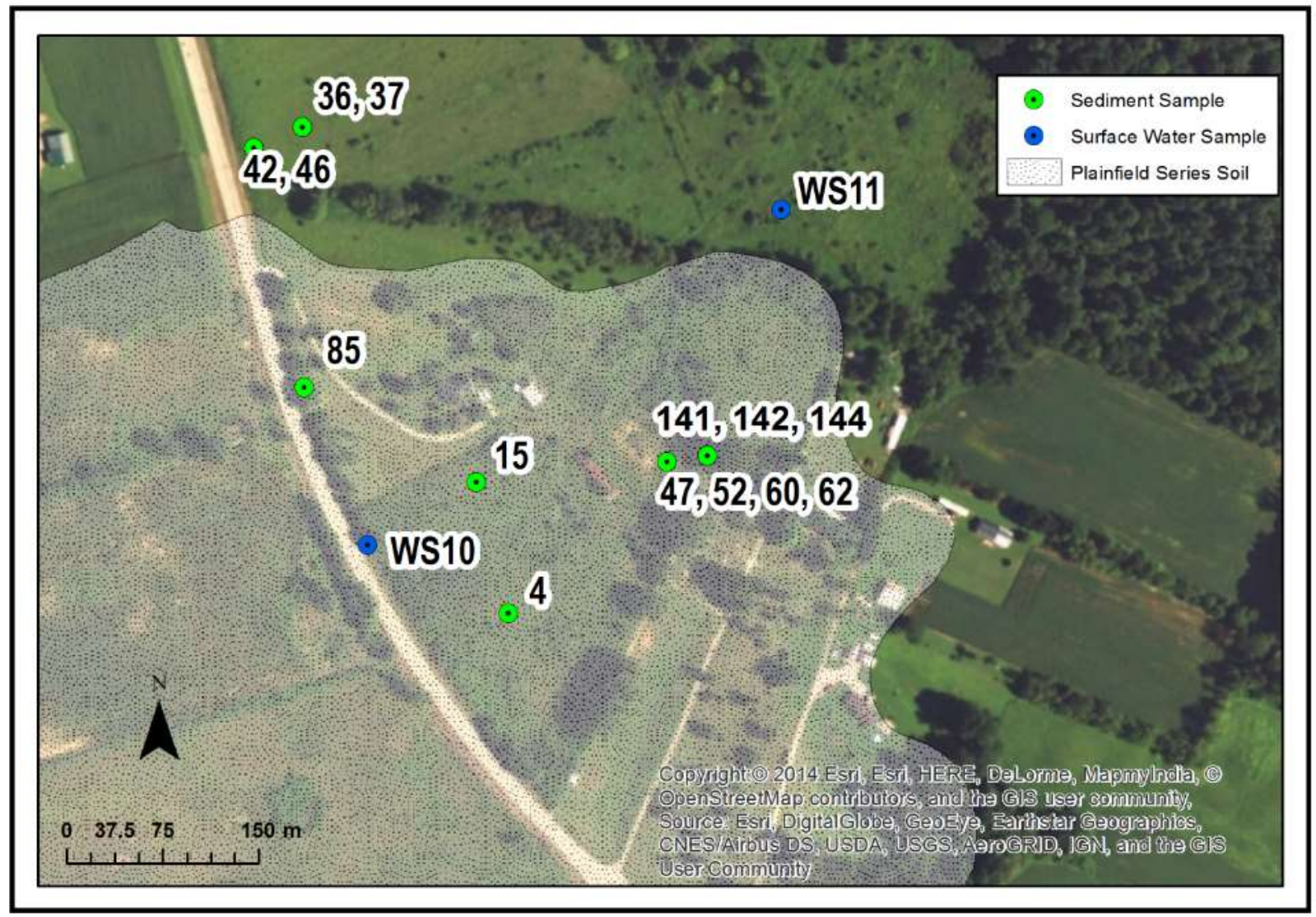

Figure 3-3. Location of sediment samples in relation to dunes and Plainfield Series Soil in Sandy Springs. See Figure 3-2 for figure location.

\section{3. $p H$}

A Mettler Toledo $\mathrm{pH}$ and conductivity meter was used to determine $\mathrm{pH}$ measurements for sediment samples. Measurements were taken on a saturated paste consisting of 1:1 deionized water to sediment ( $5 \mathrm{~g}$ of sediment: $5 \mathrm{ml}$ of deionized water) mixture. Prior to measurement, pastes were agitated and allowed to stand for $\sim 1$ hour.

Table 3-2. Description of soil types and profiles for sediment samples.

\begin{tabular}{|c|c|c|c|c|c|}
\hline Context & Code & USDA-NRCS Soil Type & Soil Profile $^{1}$ & $\begin{array}{l}\text { Sample } \\
\text { Depth (m) }\end{array}$ & $\begin{array}{l}\text { Sample } \\
\text { Texture }\end{array}$ \\
\hline Dune, Cut-bank 1/1a & 52 & $\begin{array}{l}\text { Plainfield sand, } 3 \text { to } 8 \\
\text { percent slopes }\end{array}$ & $\begin{array}{l}\mathrm{A}-\mathrm{Bw}-2 \mathrm{Bw}-3 \mathrm{Bw}-3 \mathrm{C}-4 \mathrm{C}- \\
5 \mathrm{C}-6 \mathrm{C}\end{array}$ & 1.40 & sand \\
\hline Dune, Cut-bank 1/1a & 47 & $\begin{array}{l}\text { Plainfield sand, } 3 \text { to } 8 \\
\text { percent slopes }\end{array}$ & $\begin{array}{l}\text { A-Bw-2Bw-3Bw-3C-4C- } \\
5 \mathrm{C}-6 \mathrm{C}\end{array}$ & 1.60 & sand \\
\hline
\end{tabular}




\begin{tabular}{|c|c|c|c|c|c|}
\hline Context & Code & USDA-NRCS Soil Type & Soil Profile $^{1}$ & $\begin{array}{l}\text { Sample } \\
\text { Depth (m) }\end{array}$ & $\begin{array}{l}\text { Sample } \\
\text { Texture }\end{array}$ \\
\hline Dune, Cut-bank 1/1a & 60 & $\begin{array}{l}\text { Plainfield sand, } 3 \text { to } 8 \\
\text { percent slopes }\end{array}$ & $\begin{array}{l}\text { A-Bw-2Bw-3Bw-3C-4C- } \\
5 \mathrm{C}-6 \mathrm{C}\end{array}$ & 3.45 & sand \\
\hline Dune, Cut-bank 1/1a & 62 & $\begin{array}{l}\text { Plainfield sand, } 3 \text { to } 8 \\
\text { percent slopes }\end{array}$ & $\begin{array}{l}\text { A-Bw-2Bw-3Bw-3C-4C- } \\
5 \mathrm{C}-6 \mathrm{C}\end{array}$ & 4.10 & sand \\
\hline Sand sheet, Shovel Test 5 & 4 & $\begin{array}{l}\text { Plainfield sand, } 3 \text { to } 8 \\
\text { percent slopes }\end{array}$ & A-Ap-Bw-C & 1.60 & sand \\
\hline Sand sheet, Shovel Test 24 & 15 & $\begin{array}{l}\text { Plainfield sand, } 3 \text { to } 8 \\
\text { percent slopes }\end{array}$ & A-Ap-Bw-C & 1.65 & silty sand \\
\hline Dune, Cut-bank 2/2b & 85 & $\begin{array}{l}\text { Plainfield sand, } 3 \text { to } 8 \\
\text { percent slopes }\end{array}$ & A-C1-2C2-2C3-3C4-3C5g & 3.10 & silty sand \\
\hline $\begin{array}{l}\text { Dune/Suspected seep area, } \\
\text { Giddings Probe } 6\end{array}$ & 141 & $\begin{array}{l}\text { Plainfield sand, } 3 \text { to } 8 \\
\text { percent slopes }\end{array}$ & $\begin{array}{l}\text { AC-Bw-C1-C2-2AC'- } \\
2 C^{\prime} 1-2 C^{\prime} 2\end{array}$ & 0.43 & silty sand \\
\hline $\begin{array}{l}\text { Dune/Suspected seep area, } \\
\text { Giddings Probe } 6\end{array}$ & 142 & $\begin{array}{l}\text { Plainfield sand, } 3 \text { to } 8 \\
\text { percent slopes }\end{array}$ & $\begin{array}{l}\text { AC-Bw-C1-C2-2AC'- } \\
2 C^{\prime} 1-2 C^{\prime} 2\end{array}$ & 1.52 & silty sand \\
\hline $\begin{array}{l}\text { Dune/Suspected seep area, } \\
\text { Giddings Probe } 6\end{array}$ & 144 & $\begin{array}{l}\text { Plainfield sand, } 3 \text { to } 8 \\
\text { percent slopes }\end{array}$ & $\begin{array}{l}A C-B w-C 1-C 2-2 A^{\prime}- \\
2 C^{\prime} 1-2 C^{\prime} 2\end{array}$ & 3.00 & sand \\
\hline $\begin{array}{l}\text { Tributary Alluvium, Shovel } \\
\text { Test } 61\end{array}$ & 37 & $\begin{array}{l}\text { Sciotoville silt loam, } 1 \text { to } \\
6 \text { percent slopes }\end{array}$ & Ap-Bt & 1.14 & sandy mud \\
\hline $\begin{array}{l}\text { Tributary Alluvium, Shovel } \\
\text { Test } 61\end{array}$ & 36 & $\begin{array}{l}\text { Sciotoville silt loam, } 1 \text { to } \\
6 \text { percent slopes }\end{array}$ & Ap-Bt & 1.40 & mud \\
\hline $\begin{array}{l}\text { Tributary Alluvium, Shovel } \\
\text { Test } 58\end{array}$ & 42 & $\begin{array}{l}\text { Sciotoville silt loam, } 1 \text { to } \\
6 \text { percent slopes }\end{array}$ & A-Ap-Bt-BC & 0.95 & sandy mud \\
\hline $\begin{array}{l}\text { Tributary Alluvium, Shovel } \\
\text { Test } 58\end{array}$ & 46 & $\begin{array}{l}\text { Sciotoville silt loam, } 1 \text { to } \\
6 \text { percent slopes }\end{array}$ & A-Ap-Bt-BC & 1.10 & sandy mud \\
\hline
\end{tabular}

${ }^{1}$ Bold marks soil horizon from which sediment sample was collected

\section{Results}

\subsection{Review of geological literature}

Regional bedrock geology suggests a low potential for saline springs in the area of Sandy Springs. Upper units of the Silurian Salina Group, thought to be the primary source of Ohio brines and saline waters (Carlson, 1991, pp. 11-12; M. C. Hansen, 1983, 1995; Phalen, 1919, p. 68) are absent in southwestern Ohio in the immediate vicinity of Sandy Springs (Hull, 1990). Furthermore, regional hydrological flow gradients are not conducive to the discharge of hypersaline springs in southwestern Ohio since downward flow occurs in the western part of the state and upward flow and discharge occur in the eastern section (M. C. Hansen, 1995, p. 2). This pattern led (M. C. Hansen, 1995, p. 2) to state that "salt springs, at which connate water (brine) is discharged, appear to be confined to eastern Ohio." Also, mapped geologic faults which could provide efficient pathways for deep-seated saline waters are absent within $32 \mathrm{~km}$ of Sandy Springs (see Figure 3-1).

Although the geologic literature suggests that Ohio saline springs are constrained by the distribution of evaporitic rock units, several of northern Kentucky's most prominent licks - Big Bone Lick, Upper and Lower Blue Licks, and Mays Lick - are not underlain by Salina Group formations (Potter, 2007, p. 25). This absence indicates a potential alternate salt source for at least some Ohio Valley saline springs. The source of many of these northern Kentucky licks remains uncertain, but evidence from Big Bone Lick suggests connate water in permeable Middle Cambrian Mount Simon Sandstone or Middle Ordovician St. Peter Sandstone as a 
primary source (Kenneth B. Tankersley, 2007; see also Kenneth B. Tankersley et al., 2015). Geochemical analysis of Big Bone Lick groundwater produced $\mathrm{Na}: \mathrm{Cl}$ and $\mathrm{Na}: \mathrm{Br}$ ratios suggestive of Cambrian through Silurian connate water (McCartney et al., 2005). Since Big Bone Lick is situated on the western downward limb of the Cincinnati arch and appears to have an Illinois Basin source based on geochemical analysis (McCartney et al., 2005), it likely is hydrologically unrelated to saline water east of the Cincinnati arch axis, including potential springs at Sandy Springs.

Similar to Sandy Springs, Mays Lick, and the Upper and Lower Blue Licks, are situated on the eastern limb of the Cincinnati arch and likely are subject to similar deep hydrological flow patterns. Unlike Sandy Springs, however, these licks are directly underlain by Ordovician rocks and are located within $1 \mathrm{~km}$ of mapped geologic faults (see Figure 3-1). The presence of faults may account for why saline springs occur at places such as Mays Lick and the Upper and Lower Blue Licks, but not in other settings underlain by similar bedrock stratigraphy.

\subsection{Review of historical literature}

The historical literature (e.g., Bownocker, 1906; Brown, 1999; Evans \& Stivers, 1900; M. C. Hansen, 1983, 1995; Hildreth, 1838; Jakle, 1969; Phalen, 1919; Wilber Stout, Ver Steeg, \& Lamb, 1943; Stout, Lamborn, Raymond E., Schaaf, Downs., 1932; Wilcox, 1970) is silent regarding the presence of salt licks, salt works, or buffalo traces in the immediate vicinity of Sandy Springs. It is worth mentioning that two long-term Adams County residents and avid hunters, Carmel Taylor and Ferrel Whisman, both informed the author in 2015 that they were unaware of any salt licks at Sandy Springs. Mr. Taylor even remembers, as a child, drinking 'fresh' water straight from the active seep tested for this study (WS10).

Given that a substantial eighteenth through nineteenth century buffalo trace - Alanant-owamiowee - crossed the Ohio River near present-day Maysville, Kentucky (Jakle, 1969, pp. 690-691) (see Figure 3-1), a lack of known trail systems connecting Sandy Springs to regional licks is noteworthy. The lack of documented animal trails also may, at least partially, contradict the suggestion that Sandy Springs was located along a migratory animal route that served to funnel game into the valley bottomlands (Cunningham, 1973; Gramly \& Funk, 1990, p. 16; Seeman \& Prufer, 1982, p. 157). Although mapped buffalo traces are a relatively recent phenomenon tied to the late Holocene eastern expansion of modern bison (Rostlund, 1960), Smith (1990, p. 244) has argued that these networks likely are a good analogue for a similar trail system used by Pleistocene herbivores.

Although no mention of saline springs or historical salt works, was identified at Sandy Springs proper, Seeman et al. (1994, p. 79) correctly note that Vanceburg, Kentucky, immediately across the Ohio River, possessed a commercial nineteenth century salt works referred to as the Ohio Salt Lick (Cramer, 1814, p. 107; Jakle, 1969, p. 699). An early visitor to the salt work, Andrew Ellicott, mentioned a natural salt spring located in a creek bed "about one mile from the river in the state of Kentucky" (Ellicott, 1803, p. 14). Ellicott provides little additional information regarding this salt spring and it is uncertain if it represents the same location as the commercial Ohio Salt Lick. Collins and Collins (1877, p. 467) describe the Ohio Salt Lick as containing "two salt wells, three hundred feet deep...from which this part of the state was formerly supplied with 
salt." The fact that wells were excavated to a depth of 300 feet may indicate that saline concentrations were comparatively weak at the surface, especially if compared to other prolific licks such as Big Bone Lick. Morris and Pierce (1967) also state that some water wells in the Vanceburg area are known to produce sulfate and iron-concentrated waters, but only at depths where Ohio Shale bedrock has been penetrated. Collectively, the Vanceburg literature suggests the presence of at least one natural salt lick within $\sim 1.6 \mathrm{~km}$ from the Ohio River and Sandy Springs.

\subsection{Salinity}

The results of electrical conductivity testing within $20 \mathrm{~km}$ of Sandy Springs indicate low ionic concentrations in regional water sources (Table 3-3). Surface water samples range between 48.72 and $319 \mathrm{ppm}$, with an average of $185 \mathrm{ppm}$. These samples are well under the $<1000 \mathrm{ppm}$ fresh water upper limit established by the USDA (Glasser et al., 2007, p. 35) The two Ohio River samples yielded the highest (319 ppm, WS9), and third highest (310, WS3), concentrations which likely are elevated due to modern anthropogenic inputs. If Ohio River samples are removed, tributary streams average only 161 ppm with Salt Lick Creek (WS7) in Vanceburg, Kentucky, having the highest concentration at $317 \mathrm{ppm}$. Sample WS10, an active seep that emerges from the sand sheet in Sandy Springs yields the freshest water at $48.7 \mathrm{ppm}$.

Although the sample size is small, evidence suggests that lithology weakly influences water salinity at Sandy Springs. Samples underlain by Peebles Dolomite $(\mathrm{n}=6)$ average $123 \mathrm{ppm}$; whereas samples underlain by Estill Shale $(n=3)$ and Ohio Shale $(n=4)$ average slightly higher concentrations at $263 \mathrm{ppm}$ and $219 \mathrm{ppm}$, respectively. As mentioned, some area water wells excavated into Ohio Shale bedrock possess elevated TDS concentrations (Morris \& Pierce, 1967). All salinity readings in this study, however, would be classified as "fresh" according to USDA.

Table 3-3. Results of electrical conductivity and $\mathrm{pH}$ analysis for surface water and sediment samples

\begin{tabular}{|c|c|c|c|c|c|c|}
\hline Sample Site & Code & $\begin{array}{c}\text { Distance } \\
\text { to } \\
\text { Sandy } \\
\text { Springs } \\
\text { (km) } \\
\end{array}$ & Surficial Geology & $\begin{array}{c}\text { Month and } \\
\text { Year } \\
\text { Collected }\end{array}$ & $\begin{array}{l}\text { ppm } \\
\text { TDS }\end{array}$ & pH \\
\hline \multicolumn{7}{|l|}{ Surface Water Samples } \\
\hline $\begin{array}{l}\text { Unnamed tributary next } \\
\text { to dunes }\end{array}$ & WS11 & 0.0 & $\begin{array}{l}\text { Alluvium over Peebles Dolomite } \\
\text { (Silurian) }\end{array}$ & May, 2016 & 197 & na \\
\hline Sand sheet seep & WS10 & 0.0 & $\begin{array}{c}\text { Alluvium over Peebles Dolomite } \\
\text { (Silurian) }\end{array}$ & October, 2015 & 48.7 & na \\
\hline McCall Run & WS4 & 1.0 & Peebles Dolomite (Silurian) & October, 2015 & 114 & na \\
\hline Ohio River & WS9 & 1.5 & Bedload over Estill Shale (Silurian) & October, 2015 & 319 & na \\
\hline Gilpen Run Creek & WS8 & 2.0 & Peebles Dolomite (Silurian) & October, 2015 & 137 & na \\
\hline Salt Lick Creek & WS7 & 2.4 & Bedload over Ohio Shale (Devonian) & October, 2015 & 317 & na \\
\hline Sulphur Creek & WS1 & 3.5 & Estill Shale (Silurian) & October, 2015 & 159 & na \\
\hline Sulphur Creek & WS13 & 3.9 & Ohio Shale (Devonian) & August, 2016 & 158 & na \\
\hline Ohio River & WS3 & 4.3 & Bedload over Estill Shale (Silurian) & October, 2015 & 310 & na \\
\hline
\end{tabular}




\begin{tabular}{|c|c|c|c|c|c|c|}
\hline Sample Site & Code & $\begin{array}{c}\text { Distance } \\
\text { to } \\
\text { Sandy } \\
\text { Springs } \\
\text { (km) } \\
\end{array}$ & Surficial Geology & $\begin{array}{l}\text { Month and } \\
\text { Year } \\
\text { Collected }\end{array}$ & $\begin{array}{l}\text { ppm } \\
\text { TDS }\end{array}$ & pH \\
\hline Salt Lick Creek & WS6 & 4.4 & Bedload over Ohio Shale (Devonian) & October, 2015 & 238 & na \\
\hline Upper Twin Creek & WS16 & 4.5 & Peebles Dolomite (Silurian) & August, 2016 & 99.4 & na \\
\hline Sulphur Creek & WS12 & 4.9 & Peebles Dolomite (Silurian) & August, 2016 & 143 & na \\
\hline Turkey Creek & WS2 & 19.4 & Ohio Shale (Devonian) & October, 2015 & 164 & na \\
\hline $\begin{array}{l}\text { Little Salt Creek (Scioto } \\
\text { Saline) }\end{array}$ & WS5 & 74.7 & $\begin{array}{l}\text { Bedload over Maxville Limestone } \\
\text { (Mississippian) }\end{array}$ & October, 2015 & 330 & na \\
\hline $\begin{array}{c}\text { Salt lick, Big Bone Lick } \\
\text { State Park }\end{array}$ & WS14 & 130.1 & $\begin{array}{c}\text { Alluvium over Kope Shale } \\
\text { (Ordovician) }\end{array}$ & August, 2016 & 7240 & na \\
\hline $\begin{array}{l}\text { Big Bone Creek, Big } \\
\text { Bone Lick State Park }\end{array}$ & WS15 & 130.1 & $\begin{array}{c}\text { Alluvium over Kope Shale } \\
\text { (Ordovician) }\end{array}$ & August, 2016 & 321 & na \\
\hline \multicolumn{7}{|c|}{ Sediment Samples } \\
\hline $\begin{array}{l}\text { Dune, 3C horizon, } 1.4 \mathrm{~m} \\
\text { bs, sand }\end{array}$ & 52 & 0.0 & $\begin{array}{l}\text { Alluvium over Peebles Dolomite } \\
\text { (Silurian) }\end{array}$ & $\begin{array}{l}\text { September, } \\
2015\end{array}$ & 8.39 & 5.78 \\
\hline $\begin{array}{l}\text { Dune, } 4 \mathrm{C} \text { horizon, } 1.6 \mathrm{~m} \\
\text { bs, sand }\end{array}$ & 47 & 0.0 & $\begin{array}{c}\text { Alluvium over Peebles Dolomite } \\
\text { (Silurian) }\end{array}$ & $\begin{array}{l}\text { September, } \\
2015\end{array}$ & 8.68 & 5.50 \\
\hline $\begin{array}{c}\text { Dune, } 6 \mathrm{C} \text { horizon, } 3.45 \\
\mathrm{~m} \mathrm{bs} \text {, sand }\end{array}$ & 60 & 0.0 & $\begin{array}{c}\text { Alluvium over Peebles Dolomite } \\
\text { (Silurian) }\end{array}$ & October, 2015 & 5.52 & 4.98 \\
\hline $\begin{array}{c}\text { Dune, } 6 \mathrm{C} \text { horizon, } 4.1 \mathrm{~m} \\
\text { bs, sand }\end{array}$ & 62 & 0.0 & $\begin{array}{l}\text { Alluvium over Peebles Dolomite } \\
\text { (Silurian) }\end{array}$ & $\begin{array}{l}\text { September, } \\
2015\end{array}$ & 11.4 & 4.96 \\
\hline $\begin{array}{c}\text { Sand sheet, C horizon, } \\
1.6 \mathrm{~m} \mathrm{bs} \text {, sand }\end{array}$ & 4 & 0.0 & $\begin{array}{l}\text { Alluvium over Peebles Dolomite } \\
\text { (Silurian) }\end{array}$ & May, 2015 & 9.13 & 5.63 \\
\hline $\begin{array}{l}\text { Sand sheet, C horizon, } \\
1.65 \mathrm{~m} \text { bs, silty sand }\end{array}$ & 15 & 0.0 & $\begin{array}{l}\text { Alluvium over Peebles Dolomite } \\
\text { (Silurian) }\end{array}$ & May, 2015 & 39.0 & 5.62 \\
\hline $\begin{array}{l}\text { Dune, 3C5g horizon, } 3.1 \\
\text { m bs, silty sand }\end{array}$ & 85 & 0.0 & $\begin{array}{l}\text { Alluvium over Peebles Dolomite } \\
\text { (Silurian) }\end{array}$ & $\begin{array}{l}\text { November, } \\
2015\end{array}$ & 14.9 & 5.04 \\
\hline $\begin{array}{l}\text { Dune/Seep area, AC } \\
\text { horizon, } 0.43 \mathrm{~m} \text { bs, silty } \\
\text { sand }\end{array}$ & 141 & 0.0 & $\begin{array}{l}\text { Alluvium over Peebles Dolomite } \\
\text { (Silurian) }\end{array}$ & $\begin{array}{l}\text { November, } \\
2015\end{array}$ & 21.2 & 5.16 \\
\hline $\begin{array}{c}\text { Dune/Seep area, Bw } \\
\text { horizon, } 1.52 \mathrm{~m} \text { bs, silty } \\
\text { sand }\end{array}$ & 142 & 0.0 & $\begin{array}{l}\text { Alluvium over Peebles Dolomite } \\
\text { (Silurian) }\end{array}$ & $\begin{array}{l}\text { November, } \\
2015\end{array}$ & 6.80 & 5.86 \\
\hline $\begin{array}{c}\text { Dune/Seep area, C } \\
\text { horizon, } 3 \mathrm{~m} \text { bs, sand }\end{array}$ & 144 & 0.0 & $\begin{array}{l}\text { Alluvium over Peebles Dolomite } \\
\text { (Silurian) }\end{array}$ & $\begin{array}{l}\text { November, } \\
2015\end{array}$ & 8.39 & 6.24 \\
\hline $\begin{array}{l}\text { Tributary Alluvium, Bt } \\
\text { horizon, } 1.14 \mathrm{~m} \mathrm{bs}, \\
\text { Sandy Mud }\end{array}$ & 37 & 0.0 & $\begin{array}{l}\text { Alluvium over Peebles Dolomite } \\
\text { (Silurian) }\end{array}$ & $\begin{array}{l}\text { September, } \\
2015\end{array}$ & 8.10 & 5.49 \\
\hline $\begin{array}{l}\text { Tributary Alluvium, Bt } \\
\text { horizon, } 1.4 \mathrm{~m} \text { bs, Mud }\end{array}$ & 36 & 0.0 & $\begin{array}{l}\text { Alluvium over Peebles Dolomite } \\
\text { (Silurian) }\end{array}$ & $\begin{array}{l}\text { September, } \\
2015\end{array}$ & 16.1 & 5.32 \\
\hline $\begin{array}{l}\text { Tributary Alluvium, Bt } \\
\text { horizon, } 0.95 \mathrm{~m} \mathrm{bs}, \\
\text { Sandy Mud }\end{array}$ & 42 & 0.0 & $\begin{array}{l}\text { Alluvium over Peebles Dolomite } \\
\text { (Silurian) }\end{array}$ & $\begin{array}{l}\text { September, } \\
2015\end{array}$ & 20.4 & 5.11 \\
\hline $\begin{array}{c}\text { Tributary Alluvium, BC } \\
\text { horizon, } 1.1 \mathrm{~m} \text { bs, Sandy } \\
\text { Mud }\end{array}$ & 46 & 0.0 & $\begin{array}{l}\text { Alluvium over Peebles Dolomite } \\
\text { (Silurian) }\end{array}$ & $\begin{array}{l}\text { September, } \\
2015\end{array}$ & 19.2 & 5.17 \\
\hline
\end{tabular}

Samples from surface water sources acclaimed to possess concentrated saline waters, including Little Salt Creek (Scioto Saline) and Big Bone Creek (Big Bone Lick State Park), yielded slightly higher concentrations than Sandy Springs sources at 330 and 321 ppm, respectively. Although more concentrated, these waters still are classified as fresh water. Sample WS13, which was measured just a few meters downstream of an active saline spring producing a strong 
sulfur odor at Big Bone Lick, yielded a brackish water reading of 7,240 ppm, 2000 percent greater than any Sandy Springs sample (Table 3-3). The Big Bone Lick reading reported here is similar to one reported from the Lower Blue Lick where water salinity measured 10,296 ppm in 1850 (Hopkins, 1966). Taken together, brackish water readings from licks at Big Bone Lick and Lower Blue Lick better align with expectations of a spring sufficiently saline as to attract large numbers of sodium-deprived animals.

\section{4. $p H$}

Responses of soil $\mathrm{pH}$ to cation and anion enrichment is complex and related to myriad factors of water chemistry, climate and precipitation trends, and salt composition. For example, most researchers report increasing $\mathrm{pH}$ levels in the presence of sodium chlorides, gypsum, sodium sulfates, and bicarbonates (e.g., Gupta, Singh, \& Abrol, 1989; J. J. Miller, Pawluk, \& Beke, 1993; Schaetzl \& Anderson, 2005, pp. 427-430), although at least one study reports a pH decrease in the presence of calcium carbonates (e.g., Al-Busaidi \& Cookson, 2003). Though soluble salts would not be expected to accumulate in the upper reaches of permeable sediments such as sand, at least in the humid eastern U.S., sediments located below the wetting front or proximal to supersaturated saline groundwater may result in mineral precipitation and associated soil salinization which would alter soil pH, presumably raising it (Schaetzl \& Anderson, 2005, pp. 427-430).

The 14 samples from contexts within, or adjacent, to dunes and the sand sheet yielded $\mathrm{pH}$ values in the slightly acidic range between 4.96 and 6.24, with an average of 5.40 (Table 3-2). When compared to a larger sample of 78 sediment samples from non-dune, alluvial, contexts across Sandy Springs, no statistical difference was identified as these sediments produced a $\mathrm{pH}$ range between 4.35 and 7.98, with an average of $5.40(t=0.0048 ; p=0.996)$. These $\mathrm{pH}$ ranges are well within reported county-wide values for local Plainfield and Sciotoville Soil Series (Lucht \& Brown, 1994, pp. 186-190).

\subsection{Discussion and Conclusion}

As illustrated in recent treatises on eastern U.S. Paleoindian archaeology, it is increasingly clear that many traditional models of Paleoindian chronology, settlement, economics, land-use behavior, and subsistence, require careful reevaluation (e.g., Anderson, 2013; Anderson et al., 2015; Ellis, 2011; Eren et al., 2015; Holliday \& Miller, 2014; Meltzer \& Holliday, 2010; Speth et al., 2013; Waters \& Stafford, 2007). Many current models rely heavily on data from sites that, in truth, are poorly documented or understood. This reliance has resulted in calls for increased efforts to reinvestigate classic eastern U.S. Paleoindian sites (see Gingerich, 2013b). Reinvestigation of sites such as Shoop (Carr, Adovasio, \& Vento, 2013), Bull Brook (B. S. Robinson \& Ort, 2013; B. S. Robinson, Ort, Eldridge, Burke, \& Pelletier, 2009), Plenge (Gingerich, 2013a), and Shawnee-Minisink (Gingerich, 2013c) has provided new insights into intra-site organization and in many cases corrected enduring inaccuracies.

The results of electrical conductivity testing of surface water and sediment samples, $\mathrm{pH}$ testing of sediment samples, and review of relevant geological and historical literature, fail to confirm Cunningham's often cited claim of high salinity for area groundwater and streams at Sandy 
Springs. Geologically, Sandy Springs is not underlain by shallow evaporite rock units nor are significant geologic faults mapped for the area. TDS values of tested water samples within $20 \mathrm{~km}$ of Sandy Springs yielded concentrations between 48.7 and 320 ppm, all well below USDA's brackish water threshold of 1,000 ppm (Glasser et al., 2007, p. 35)). These values also are far below reported values for known salt licks at Big Bone Lick (7,240 ppm, this study) and Lower Blue Licks (10,296 ppm; Hopkins, 1966, p. 2). Finally, no mention of commercial salt wells or animal trails such as buffalo traces was located in the literature for Adams County, Ohio.

Although this study failed to identify evidence of surficial saline waters at Sandy Springs proper, historical evidence suggests that a natural saline spring was situated approximately $1.6 \mathrm{~km}$ south of the Ohio River near the modern town of Vanceburg, Kentucky (Ellicott, 1803, p. 43). It is possible that historic accounts of this spring may be the source of Cunningham's initial claim of hypersaline waters. The absence of known animal trails or buffalo traces connecting this lick to other documented licks such as Lower Blue Licks or Big Bone Lick (see Figure 3-1), fail to suggest a spring of sufficient size or salinity to attract substantial numbers of game or subsequent human predators. Indeed, the simple fact that Paleoindian material is concentrated at Sandy Springs, Ohio, and not $\sim 2 \mathrm{~km}$ away at Vanceburg, Kentucky, suggests that Ellicot's reported lick was only a minor resource during the late Pleistocene.

The possible absence of saline springs from Sandy Springs severely undermines a key component in the narrative that has surrounded the Paleoindian occupation of the site since Cunningham's 1973 article. As initially claimed, the presence of saline waters and subsequent concentration of sodium-deprived game helped explain why Sandy Springs was repeatedly, and intensively, occupied by Paleoindians despite being located in an unglaciated landscape far removed from high-quality bedded chert sources (Lepper \& Meltzer, 1991)). Without the presence of saline springs, it is difficult to offer a reason as to why hunted game would repeatedly concentrate at Sandy Springs in the first place. Accordingly, the abundance of projectile points at Sandy Springs, although clearly indicative of a population heavily invested in hunting strategies (Seeman et al., 1994, p. 83), does not necessarily equate to a site that functioned as a hunting station. In this view, the presence of projectile points at Sandy Springs may be incidental, perhaps simply reflecting the high archaeological visibility of chert artifacts upon a persistently reoccupied late Pleistocene landscape. The possibility that additional resources, including non-economic ones (e.g., see Speth et al., 2013), may have attracted late Pleistocene groups to Sandy Springs is considered in the remainder of this article.

Aside from hunting, I offer two broad hypotheses that may account for the persistent occupation of Sandy Springs by Paleoindians. First, Sandy Springs is characterized by a remnant sand prairie ecosystem which contains a range of rare plant and animal species (Ohio Department of Natural Resources, 2016). Assuming that this ecosystem dates to the late Pleistocene in some form, it is possible that this setting contained desirable resources otherwise difficult to obtain in the upper Ohio River Valley. One possible plant resource that could have been targeted by Paleoindians is the eastern prickly pear cactus (Opuntia humifusa) which was observed to be a prolific colonizer of sand dunes at Sandy Springs. This succulent is known to possess various pharmaceutical benefits including use as an anti-inflammatory agent, and also produces edible foliage and fruits (Abella \& Jaeger, 2004). O. humifusa was used by various historical Native American tribes for both medicinal and subsistence reasons (Gilmore, 1919; Moerman, 1988) 
and plant remains have been found within archaeological middens at several eastern U.S. sites (e.g., McAvoy \& Harrison, 2012; Moseley, 1931).

A second potential reason as to why Sandy Springs was persistently occupied may be related to the initial efforts of wayfinding, trail establishment, and cognitive map development that accompanies early attempts at landscape learning by colonizing populations (Golledge, 2003; Kelly, 2003; Meltzer, 2003). As part of the landscape learning process, preliterate huntergatherer societies are known to focus initially on landforms that are visually unique, easy to identify, and easy to relocate. Through naming and myth creation, such localities become imbued with social meaning which aids in mnemonic learning of new landscapes (see Kelly, 2003, pp. 45-48). Places also may attain significance if they are the scene of dramatic events, especially ones that may reinforce important social norms or practices (e.g., Biesele, 1993, pp. 55-56). Miller (2016, p. 712) also suggests that distinctive locations would have been ideal for periodic aggregation of dispersed Paleoindian populations in the southeast as a way to build, and maintain, social relationships. It also has long been suggested that bedded chert outcrops would have similarly attracted eastern U.S. Paleoindians where socially important information, goods, and perhaps people (i.e., marriage partners), could be exchanged among otherwise dispersed communities (Ellis, 2011; e.g., Eren \& Desjardine, 2015; Gardner, 1977; Smallwood, 2012).

I suggest that Sandy Springs may have served in a similar manner for late Pleistocene groups early in the landscape learning process. Although Sandy Springs is not located close to bedded chert outcrops, the hummocky landscape contains large sand dunes and, coupled with the presence of a xeric biome, the site would have been visually distinctive and easy to describe to others. The visibility of raised dunes may have been enhanced further by an open, grassy, savannah-like environment that, based on pollen data, may have persisted on this broad meanderbelt during this time (see Purtill, 2012, pp. 42-43).

Relatedly, Sandy Springs may have gained prominence among late Pleistocene groups by being strategically located along an early northeast-to-southwest trail system. Seeman \& Prufer (1982, p. 157) initially discussed this possibility and suggested that Sandy Springs may have been situated along a route connecting two areas of high Paleoindian site densities: Tennessee and Cumberland drainages and the upper Ohio River drainage (Anderson, 1990, 1995; Anderson et al., 2015). The documentation of at least 15 Cumberland points from Sandy Springs (Seeman et al., 1994) implies a southerly connection since this point style is geographically restricted to the southeast (Tune, 2106). According to Tune (2016, p. 311), Sandy Springs is located near the northern-most extension of the Cumberland point geographic distribution (Figure 3-4) and he suggests that the site may have functioned as an aggregation location for seasonally dispersed groups ((Tune, 2016, p. 314) (Figure 3-4).

If long-distance travels, perhaps as part of seasonal logistical forays (Boulanger \& Lyman, 2014; Ellis, 2011; Eren \& Redmond, 2011), were occurring between the Tennessee-Cumberland and upper Ohio drainages, one potential route would have been along the Cumberland Escarpment which separates the low-relief Interior Low Plateaus from the elevated, deeply dissected, Appalachian Plateaus Provinces (Fenneman \& Johnson, 1946) (Figure 3-4). This southwest-tonortheast trending escarpment is represented by a series of knobs and ridges that range in relief from $\sim 125 \mathrm{~m}$ in northeastern Kentucky to as high as $600 \mathrm{~m}$ in southern Tennessee, northern 
Alabama, and northwestern Georgia (Simpson \& Florea, 2009, p. 71). The high-relief terrain would have provided a prominent landform to guide travel and several researchers have indicated that physiographic boundary areas were especially attractive to Paleoindians (e.g., Shane Miller, 2016, p. 710; Tune, 2016, p. 311). Significantly, groups following the western escarpment edge northward from the Tennessee and Cumberland drainages would arrive at the upper Ohio River within $10 \mathrm{~km}$ of Sandy Springs (Figure 3-4) where a natural ford could facilitate river crossing (see Seeman et al., 1994, p. 79) The presence of Paleoindian points manufactured from Upper Mercer chert at Sandy Springs (Seeman et al., 1994, p. 81), which crops out to the northeast in east-central Ohio (Wilbur Stout \& Schoenlaub, 1945, pp. 39-60), also indicates a northeasterly connection for the area as well. It is possible that Sandy Springs was strategically positioned along a north-south trail system that, once established early in Paleoindian times, became incorporated into a cognitive map of the region and continued to be used throughout the Paleoindian period. This interpretation fits well with current views of eastern U.S. Paleoindian societies being socially interconnected over broad distances, especially with regards to aspects of chert acquisition (Boulanger et al., 2015; Ellis, 2011; Eren \& Desjardine, 2015; Eren \& Redmond, 2011; Speth et al., 2013). It also may suggest that Sandy Springs, and perhaps other upper Ohio River Paleoindian sites, had occupants more aligned culturally with southern Paleoindian societies than northern ones, at least during the period of Cumberland point manufacture (12,800-12,100 cal yr BP; Tune, 2016, p. 312).

Finally, a word of caution is offered regarding the potential role of salt licks and late Pleistocene hunters. Even at renowned Pleistocene salt licks such as Big Bone Lick in Kentucky and Saltville in Virginia, the possibility that Paleoindian material and Pleistocene megafauna are contemporary or directly associated is tenuous and poorly supported (McCary, 1951, p. 11; Kenneth B. Tankersley, 1996, p. 28). This fact, coupled with the results of this study, reemphasizes the importance of critically reevaluating data from many of the sites we already know since they often form the foundation for many current models of eastern U.S. Paleoindian behavior. 


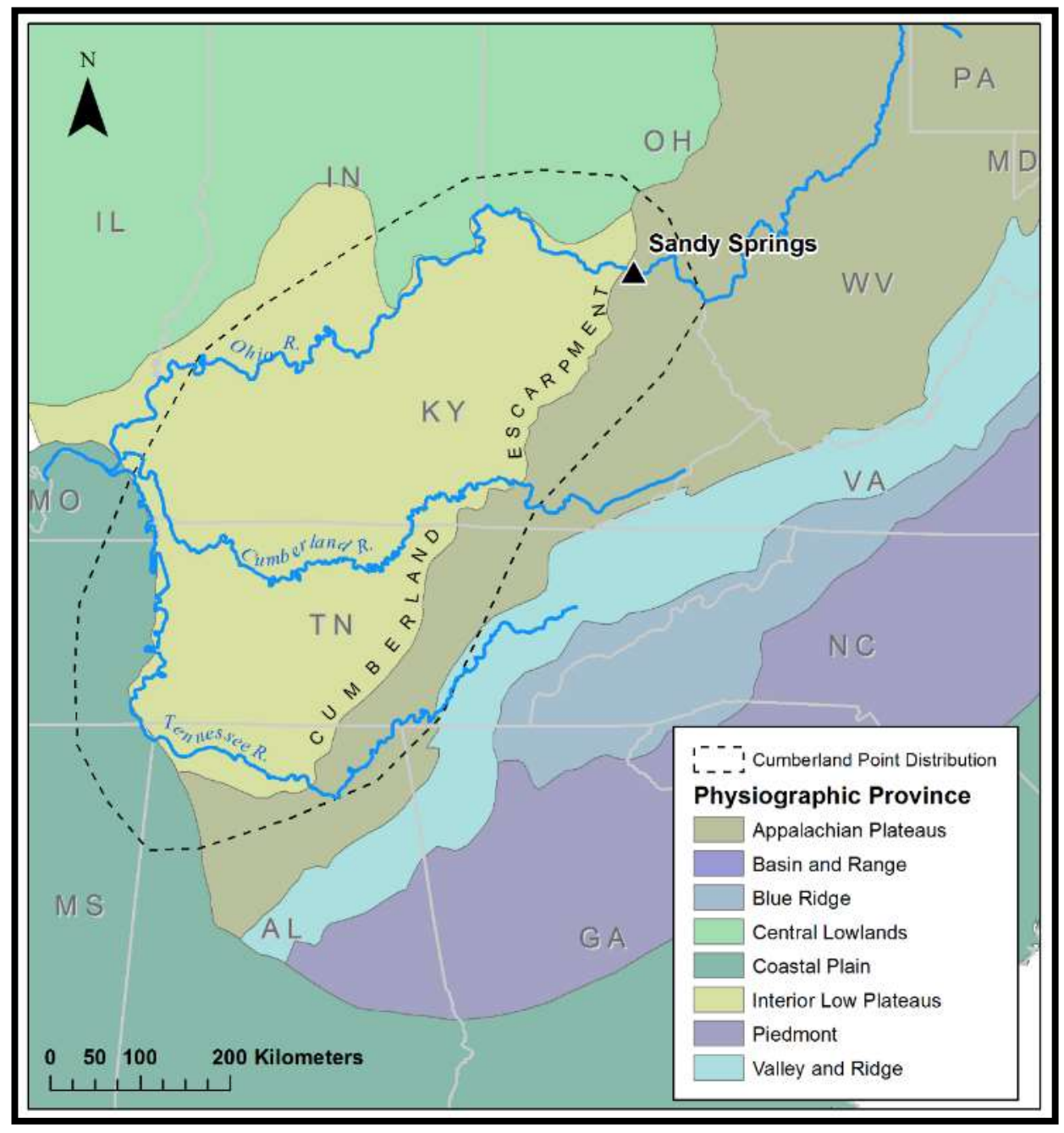

Figure 3-4. Location of Sandy Springs in relation to the Cumberland Escarpment and its position at the northern distribution of Cumberland projectile points (following Tune, 2016, p. 311).

\section{Acknowledgements}

Funding from the 2016 NASA West Virginia Space Grant Consortium Graduate Research Fellowship and the Eberly College of Arts and Sciences, Doctoral Student Research Program, West Virginia University, supported various aspects of this research. Several individuals provided project assistance including J. Steven Kite and Joseph Donovan of West Virginia University; Mark Seeman of Kent State University; Steve Baker of USDA-NRCS; Mike Angle, Frank Fugitt, and Nathan Erber of the Ohio Geological Survey; James Thompson of West Virginia University; Chris Bedel of the Edge of Appalachia Preserve System; and Nancy Stranahan of the Arc of Appalachia. The following also assisted in field and laboratory efforts: Francesca Basil; Andrew Braun; Nick Dadamo; Sara DeAloia; Lena Fox, Nancy Gostic; Zach Haidar; Heather Jewel; Matt Koerner; Miles Reed; Emily Swaney; and Joey Zampayo. Special thanks go to the Adams, Rutledge, and Whisman families for providing property access. Finally, review comments provided by two anonymous reviewers improved the quality of this article. 
Chapter 3. Non-journal Article Supplemental Information

In addition to the reevaluation of the role of saline water in the occupation of Sandy Springs presented in the journal article, research also generated data regarding the Sandy Springs artifact assemblage not included in the final journal submission. This supplement summarizes these findings and forwards preliminary conclusions. This information may be applied to an array of issues including better delineation of National Register boundaries, identification of intrasite activity areas, and a better understanding of site function.

A total of 6003 chipped-stone artifacts from a 1976 archaeological survey at Sandy Springs (Chapman \& Otto, 1976) and 399 artifacts from 2015-16 dissertation survey and excavation are analyzed as part of this research (Tables S8-S10). This reanalysis focuses on chipped-stone artifacts from the 1976 research and includes 88 Refined, Hafted Biface types identifiable to a specific temporal period (Figure S6). Significantly, two previously unidentified Paleoindian Refined, Hafted Bifaces (Clovis and Cumberland) are identified in the 1976 collection. Moreover, this collection yields two additional chipped-stone tool fragments that may also represent Paleoindian Refined, Hafted Bifaces but are too fragmented to accurately identify.

Several tool types and artifacts potentially Paleoindian in origin are identified (Figures S7-S13). Assignment of individual artifacts to the Paleoindian period is based on a combination of factors including consideration of techno-morphological traits, raw material use, and flaking pattern (Justice, 1987; Kraft, 1973; Seeman \& Prufer, 1982; Seeman et al., 1994; Smith Jr, 1995). Preference for high-quality, bedded Upper Mercer and Vanport cherts from Ohio, and Harrison County (Wyandotte) and Attica cherts from Indiana is well established for Paleoindian populations (Anderson, 1995; Mullett, 2009; Seeman, 1994; Seeman et al., 1994; Kenneth B. Tankersley et al., 1990). At Sandy Springs, Vanport and Harrison County cherts were also used to manufacture seven Middle Woodland lamellar bladelets and one Late Archaic Refined, Hafted Biface (Matanzas Side Notched). Upper Mercer chert also was used to manufacture artifacts from the Late Archaic (Brewerton Side Notched) and Middle Woodland (Snyders Cluster) periods. Although this analysis indicates a potential that fine-grained cherts such as Vanport, Upper Mercer, or Harrison County were used to manufacture post-Paleoindian tools, the presence of Upper Mercer at Sandy Springs is still considered a potential indicator of Paleoindian activity based on results from previous research (Meltzer, 2009; Mullett, 2009; Prufer \& Baby, 1963; Kenneth B. Tankersley \& Isaac, 1990; Werner et al., 2017). Upper Ohio Valley collections tend to be poor in Upper Mercer chert as it typically accounts for less than 1\% of area artifact assemblages (e.g., Purtill, 2012). At some Sandy Springs sites, it high proportions (e.g., Site $33 \mathrm{AD} 30=\sim 12 \%$ ) suggest deliberate preference of this material by some prehistoric occupants.

The spatial distribution of several dominate chert types in the 1976 collection (Upper Mercer, Vanport, Brassfield, Harrison County, Newman, Paoli, and Boyle) are mapped to determine if spatial patterning across Sandy Springs can be detected (Figures S14-S20). Mapping is based on relative proportions of chert types extrapolated from each archaeological site centerpoint $(n=26)$. 
This approach is done since more systematic approaches such as transect survey or piece plotting were not employed during the 1976 survey (Chapman \& Otto, 1976). To provide some insight into the patterning of potential Paleoindian sites across the Sandy Springs landscape, artifact densities were mapped by site centerpoints with proportional symbols. These symbols were then shown on a map with the 1976 survey boundaries illustrated. A few sites occur outside reported survey boundaries (e.g., 33AD105) and it is unclear if artifacts associated with these sites were recovered during the 1976 surface survey or by other means (e.g., recovered from amateur collectors).

In 2015-16, new survey and excavation was undertaken on properties to the north of U.S. 52 to determine if site boundaries (e.g., 33AD30, 33AD92) extend into the sand dune and sand sheet areas (Figure S16-S17). Excavations were supplemented with limited surface collection of the Adams Family property. The focus of this surface collection was to recovery artifacts of potential Paleoindian origin. Hence, only artifacts manufactured from fine-grained material such as Harrison County and Upper Mercer cherts were collected (Figures S14, S16).

The reanalysis of the 1976 collection and 2015-16 investigation allows for the advancement of several preliminary conclusions regarding Sandy Springs:

- The distribution of artifacts and material types (Upper Mercer) of suspected Paleoindian origin suggest that the initial 1974 National Register boundaries drawn for the "Adams County Paleo-Indian District" (Drennen, 1974) require substantial revision. Although accurate site boundaries remain uncertain, artifacts thought to be associated with Paleoindian occupation extend up to $0.7 \mathrm{~km}$ outside of the current National Register boundary to the north, east, and south.

- Tools manufactured from specific raw material types fail to demonstrate homogenous spatial patterning across the landscape. Noteworthy is the high concentration of Upper Mercer chert at Site 33AD30, long considered to be the main focus of the Paleoindian activity at Sandy Springs.

- In his initial discussion of Sandy Springs, Cunningham (1973) posited that some Paleoindian artifacts were recovered from "within" sand dunes suggesting the potential for site burial by aeolian sedimentation during segments of the Holocene. Based on Holocene ages revealed by OSL dating of landforms (see Chapter 4), Cunningham's statement remains potentially valid on both the S2 and S3 landforms.

- Cunningham (1973) also suggested that Paleoindian occupation occurred within a series of discrete occupations, or hot spots, across the landscape although in close proximity to dunes. This study supports this notion as suspected Paleoindian artifacts are distributed across the landscape into several apparent clusters. Further survey would be needed to delineated suspected clusters.

- Seeman and colleagues identified 98 Paleoindian Refined, Hafted Bifaces in amateur collections from Sandy Springs (Seeman et al., 1994). To this inventory, the 1976 collection contains at least two additional artifacts (Clovis and Cumberland). 
- Although rare, the 1976 collection did contain several spurred endscrapers that appear morphologically similar to ones common in more northern assemblages (Seeman, 1994; Seeman, Loebel, Comstock, \& Summers, 2013). This may indicate some hide processing occurred at Sandy Springs.

This reanalysis has demonstrated that much new information can be garnered from collections that already exist at Sandy Springs. Analyses such as this should continue to be conducted on existing collections to see what new information can be gleaned.

Table S8. Artifact inventory from Sandy Springs

\begin{tabular}{|c|c|c|c|}
\hline & 2015-16 Collection & 1976 Collection & Total \\
\hline Chipped Stone tool & 7 & 603 & 610 \\
\hline End Scraper & 1 & 83 & 84 \\
\hline indet Biface & - & 138 & 138 \\
\hline Indet Uniface & - & 47 & 47 \\
\hline Refined, Hafted Biface & 4 & 175 & 179 \\
\hline Refined, Non-hafted Biface & - & 38 & 38 \\
\hline Side Scraper & - & 61 & 61 \\
\hline Unrefined Biface & 2 & 61 & 63 \\
\hline Core & 1 & 204 & 205 \\
\hline Conical core & - & 6 & 6 \\
\hline Discoidal Core & - & 2 & 2 \\
\hline Irregular core & 1 & 195 & 196 \\
\hline Spherical Core & - & 1 & 1 \\
\hline Debitage & 85 & 5196 & 5281 \\
\hline Complete Flake & 25 & 1348 & 1373 \\
\hline Debris & - & 520 & 520 \\
\hline Distal FF & 35 & 1862 & 1897 \\
\hline Proximal FF & 25 & 1466 & 1491 \\
\hline FCR & 305 & - & 305 \\
\hline (blank) & 305 & - & 305 \\
\hline Hammerstone & 1 & - & 1 \\
\hline (blank) & 1 & - & 1 \\
\hline Grand Total & 399 & 6003 & 6402 \\
\hline
\end{tabular}

Table S9. Raw material chert type proportions by site for the Sandy Springs 1976 and 2015-16 collections

\begin{tabular}{|l|l|l|l|l|l|l|l|l|l|l|l|}
\hline Site & Brass. & Van. & UM. & Del. & Pa. & New. & Bo. & KB. & HC. & Oth. & Total \\
\hline $33 \mathrm{AD} 30$ & $9.27 \%$ & $1.32 \%$ & $11.92 \%$ & $0.00 \%$ & $11.92 \%$ & $19.87 \%$ & $0.66 \%$ & $1.32 \%$ & $4.64 \%$ & $39.07 \%$ & $100.00 \%$ \\
\hline $33 \mathrm{AD} 79$ & $6.92 \%$ & $0.00 \%$ & $0.00 \%$ & $1.26 \%$ & $15.72 \%$ & $22.64 \%$ & $0.00 \%$ & $0.00 \%$ & $0.00 \%$ & $53.46 \%$ & $100.00 \%$ \\
\hline $33 \mathrm{AD} 80$ & $7.66 \%$ & $0.00 \%$ & $0.19 \%$ & $0.38 \%$ & $25.48 \%$ & $24.52 \%$ & $0.77 \%$ & $0.96 \%$ & $1.53 \%$ & $38.51 \%$ & $100.00 \%$ \\
\hline $33 \mathrm{AD} 81$ & $13.65 \%$ & $0.16 \%$ & $0.16 \%$ & $0.48 \%$ & $9.37 \%$ & $40.32 \%$ & $0.48 \%$ & $0.95 \%$ & $0.32 \%$ & $34.13 \%$ & $100.00 \%$ \\
\hline
\end{tabular}




\begin{tabular}{|c|c|c|c|c|c|c|c|c|c|c|c|}
\hline Site & Brass. & Van. & UM. & Del. & Pa. & New. & Bo. & KB. & HC. & Oth. & Total \\
\hline 33AD82 & $15.60 \%$ & $0.20 \%$ & $0.20 \%$ & $3.20 \%$ & $10.20 \%$ & $41.60 \%$ & $0.20 \%$ & $0.80 \%$ & $0.20 \%$ & $27.80 \%$ & $100.00 \%$ \\
\hline 33AD83 & $3.45 \%$ & $0.00 \%$ & $6.90 \%$ & $3.45 \%$ & $17.24 \%$ & $20.69 \%$ & $3.45 \%$ & $0.00 \%$ & $6.90 \%$ & $37.93 \%$ & $100.00 \%$ \\
\hline 33AD84 & $11.79 \%$ & $0.00 \%$ & $0.42 \%$ & $1.05 \%$ & $9.05 \%$ & $34.32 \%$ & $0.00 \%$ & $0.42 \%$ & $0.00 \%$ & $42.95 \%$ & $100.00 \%$ \\
\hline $33 \mathrm{AD} 85$ & $15.93 \%$ & $0.00 \%$ & $0.16 \%$ & $0.65 \%$ & $3.74 \%$ & $39.02 \%$ & $0.00 \%$ & $1.30 \%$ & $0.16 \%$ & $39.02 \%$ & $100.00 \%$ \\
\hline 33AD86 & $16.22 \%$ & $0.00 \%$ & $0.42 \%$ & $1.41 \%$ & $8.46 \%$ & $24.40 \%$ & $0.00 \%$ & $0.71 \%$ & $0.00 \%$ & $48.38 \%$ & $100.00 \%$ \\
\hline 33AD87 & $20.00 \%$ & $1.14 \%$ & $0.00 \%$ & $2.29 \%$ & $12.00 \%$ & $19.43 \%$ & $0.57 \%$ & $2.86 \%$ & $0.00 \%$ & $41.71 \%$ & $100.00 \%$ \\
\hline 33AD88 & $14.47 \%$ & $1.32 \%$ & $0.66 \%$ & $0.66 \%$ & $13.82 \%$ & $15.13 \%$ & $2.63 \%$ & $3.29 \%$ & $0.66 \%$ & $47.37 \%$ & $100.00 \%$ \\
\hline 33AD89 & $7.46 \%$ & $1.99 \%$ & $1.49 \%$ & $1.49 \%$ & $16.92 \%$ & $27.36 \%$ & $0.00 \%$ & $1.00 \%$ & $1.49 \%$ & $40.80 \%$ & $100.00 \%$ \\
\hline $33 \mathrm{AD} 90$ & $19.67 \%$ & $1.64 \%$ & $3.28 \%$ & $1.64 \%$ & $13.11 \%$ & $18.03 \%$ & $0.00 \%$ & $0.00 \%$ & $0.00 \%$ & $42.62 \%$ & $100.00 \%$ \\
\hline 33AD91 & $14.53 \%$ & $0.00 \%$ & $0.43 \%$ & $0.43 \%$ & $24.36 \%$ & $23.93 \%$ & $0.00 \%$ & $0.00 \%$ & $0.00 \%$ & $36.32 \%$ & $100.00 \%$ \\
\hline 33AD92 & $10.49 \%$ & $2.78 \%$ & $0.93 \%$ & $1.23 \%$ & $16.67 \%$ & $29.01 \%$ & $0.00 \%$ & $0.93 \%$ & $0.31 \%$ & $37.65 \%$ & $100.00 \%$ \\
\hline 33AD94 & $0.00 \%$ & $0.00 \%$ & $0.00 \%$ & $0.00 \%$ & $0.00 \%$ & $50.00 \%$ & $0.00 \%$ & $0.00 \%$ & $0.00 \%$ & $50.00 \%$ & $100.00 \%$ \\
\hline 33AD95 & $22.73 \%$ & $0.00 \%$ & $0.00 \%$ & $0.00 \%$ & $18.18 \%$ & $22.73 \%$ & $9.09 \%$ & $0.00 \%$ & $0.00 \%$ & $27.27 \%$ & $100.00 \%$ \\
\hline $33 \mathrm{AD} 96$ & $12.20 \%$ & $0.00 \%$ & $4.88 \%$ & $0.00 \%$ & $4.88 \%$ & $31.71 \%$ & $0.00 \%$ & $0.00 \%$ & $2.44 \%$ & $43.90 \%$ & $100.00 \%$ \\
\hline 33AD97 & $11.54 \%$ & $0.00 \%$ & $0.00 \%$ & $0.00 \%$ & $23.08 \%$ & $23.08 \%$ & $0.00 \%$ & $0.00 \%$ & $3.85 \%$ & $38.46 \%$ & $100.00 \%$ \\
\hline 33AD98 & $19.00 \%$ & $1.08 \%$ & $2.15 \%$ & $2.51 \%$ & $13.26 \%$ & $19.00 \%$ & $2.87 \%$ & $0.36 \%$ & $3.23 \%$ & $36.56 \%$ & $100.00 \%$ \\
\hline 33AD99 & $12.96 \%$ & $5.56 \%$ & $5.56 \%$ & $0.00 \%$ & $16.67 \%$ & $14.81 \%$ & $3.70 \%$ & $1.85 \%$ & $0.00 \%$ & $38.89 \%$ & $100.00 \%$ \\
\hline $\begin{array}{l}\text { 33AD10 } \\
0\end{array}$ & $20.22 \%$ & $0.56 \%$ & $0.00 \%$ & $0.56 \%$ & $17.98 \%$ & $21.91 \%$ & $0.00 \%$ & $0.56 \%$ & $0.56 \%$ & $37.64 \%$ & $100.00 \%$ \\
\hline $\begin{array}{l}33 \mathrm{AD} 10 \\
2\end{array}$ & $29.69 \%$ & $0.00 \%$ & $0.44 \%$ & $2.62 \%$ & $12.23 \%$ & $17.90 \%$ & $0.00 \%$ & $0.44 \%$ & $0.87 \%$ & $35.81 \%$ & $100.00 \%$ \\
\hline $\begin{array}{l}33 \mathrm{AD} 10 \\
3\end{array}$ & $16.54 \%$ & $2.36 \%$ & $0.79 \%$ & $1.57 \%$ & $8.66 \%$ & $22.05 \%$ & $0.79 \%$ & $3.94 \%$ & $0.00 \%$ & $43.31 \%$ & $100.00 \%$ \\
\hline $\begin{array}{l}33 \mathrm{AD} 10 \\
4\end{array}$ & $22.95 \%$ & $0.00 \%$ & $0.00 \%$ & $1.64 \%$ & $14.75 \%$ & $19.67 \%$ & $1.64 \%$ & $0.00 \%$ & $0.00 \%$ & $39.34 \%$ & $100.00 \%$ \\
\hline $\begin{array}{l}33 \mathrm{AD} 10 \\
5\end{array}$ & $20.00 \%$ & $2.86 \%$ & $2.86 \%$ & $2.86 \%$ & $8.57 \%$ & $25.71 \%$ & $0.00 \%$ & $0.00 \%$ & $0.00 \%$ & $37.14 \%$ & $100.00 \%$ \\
\hline $\begin{array}{l}\text { Grand } \\
\text { Total }\end{array}$ & $\begin{array}{l}14.51 \\
\%\end{array}$ & $0.55 \%$ & $0.88 \%$ & $1.25 \%$ & $12.56 \%$ & $28.82 \%$ & $0.48 \%$ & $0.93 \%$ & $0.67 \%$ & $39.33 \%$ & $100.00 \%$ \\
\hline
\end{tabular}

Bo = Boyle chert; Brass. = Brassfield chert; Del. = Delaware chert; Van. = Vanport; New. = Newman chert; Pa. = Paoli chert; Oth. $=$ unknown chert. 


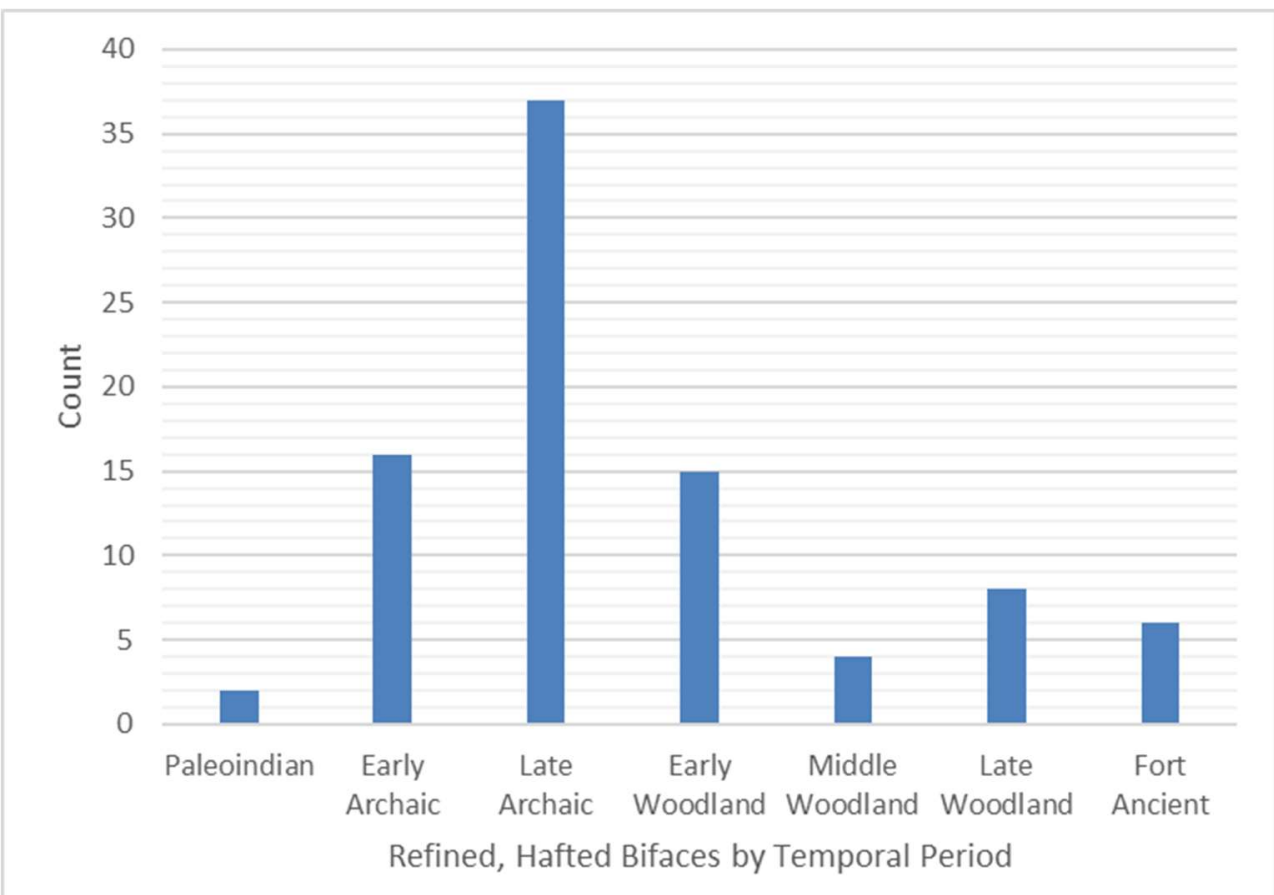

Figure S6. Inventory of Refined, Hafted Biface types by temporal period at Sandy Springs $(\mathrm{n}=88)$

Table S10. Raw material frequencies by time period

\begin{tabular}{|l|r|r|r|r|r|r|r|r|r|r|r|}
\hline & Bo. & Brass. & C-G. & Del. & New. & Pa. & MiSa. & UM. & Van. & Unk. & Total \\
\hline Paleoindian & 1 & - & - & - & - & - & - & 1 & - & - & 2 \\
\hline Early Archaic & - & 2 & 1 & - & 7 & 3 & 1 & - & - & 2 & 16 \\
\hline Late Archaic & 2 & 13 & 1 & 2 & 9 & - & - & 1 & 1 & 8 & 37 \\
\hline Early Woodland & 2 & 3 & - & 1 & 4 & 2 & - & - & - & 3 & 15 \\
\hline Middle Woodland & - & - & - & - & 2 & - & - & 1 & - & 1 & 4 \\
\hline Late Woodland & - & - & - & - & 4 & 1 & - & - & - & 3 & 8 \\
\hline Fort Ancient & - & 1 & - & 1 & 3 & - & - & - & - & 1 & 6 \\
\hline Total & $\mathbf{5}$ & $\mathbf{1 9}$ & $\mathbf{2}$ & $\mathbf{4}$ & $\mathbf{2 9}$ & $\mathbf{6}$ & $\mathbf{1}$ & $\mathbf{3}$ & $\mathbf{1}$ & $\mathbf{1 8}$ & $\mathbf{8 8}$ \\
\hline
\end{tabular}

Bo = Boyle chert; Brass. = Brassfield chert; C-G. = Cedarville-Guelph chert; Del. = Delaware chert; New. = Newman chert; Pa.

$=$ Paoli chert; MiSa. $=$ micaceous sandstone; UM. = Upper Mercer; Van. = Vanport; Unk. = unknown chert. 


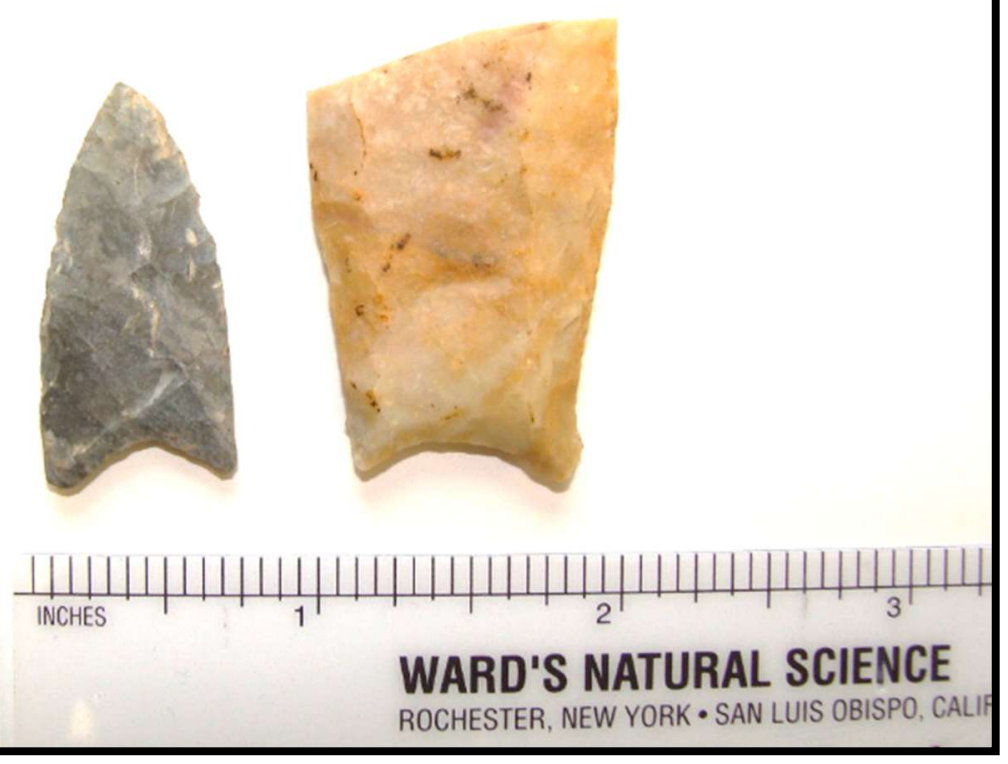

Figure S7. Left: Upper Mercer Clovis refined hafted (cat. \# 3) recovered from Site 33AD30. Right: Boyle Cumberland refined hafted biface fragment (cat. \# 366) recovered from Site 33AD87.

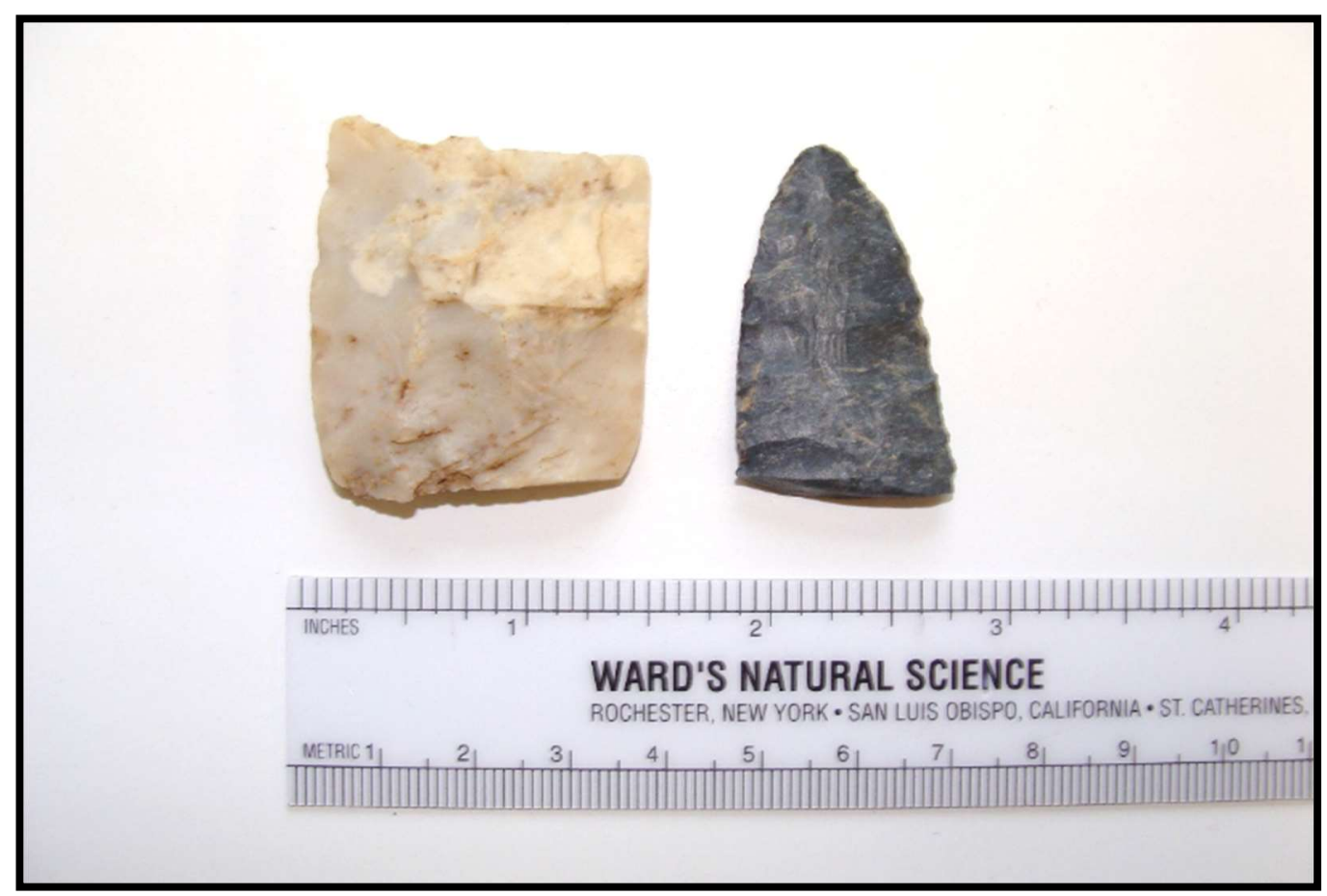

Figure S8. Left: Vanport refined hafted biface fragment (cat. \# 762) from unknown provenience (possibly Roger Cunningham donated collection). Right: Upper Mercer indeterminate biface fragment (cat. \# 4) recovered from Site 33AD89. 


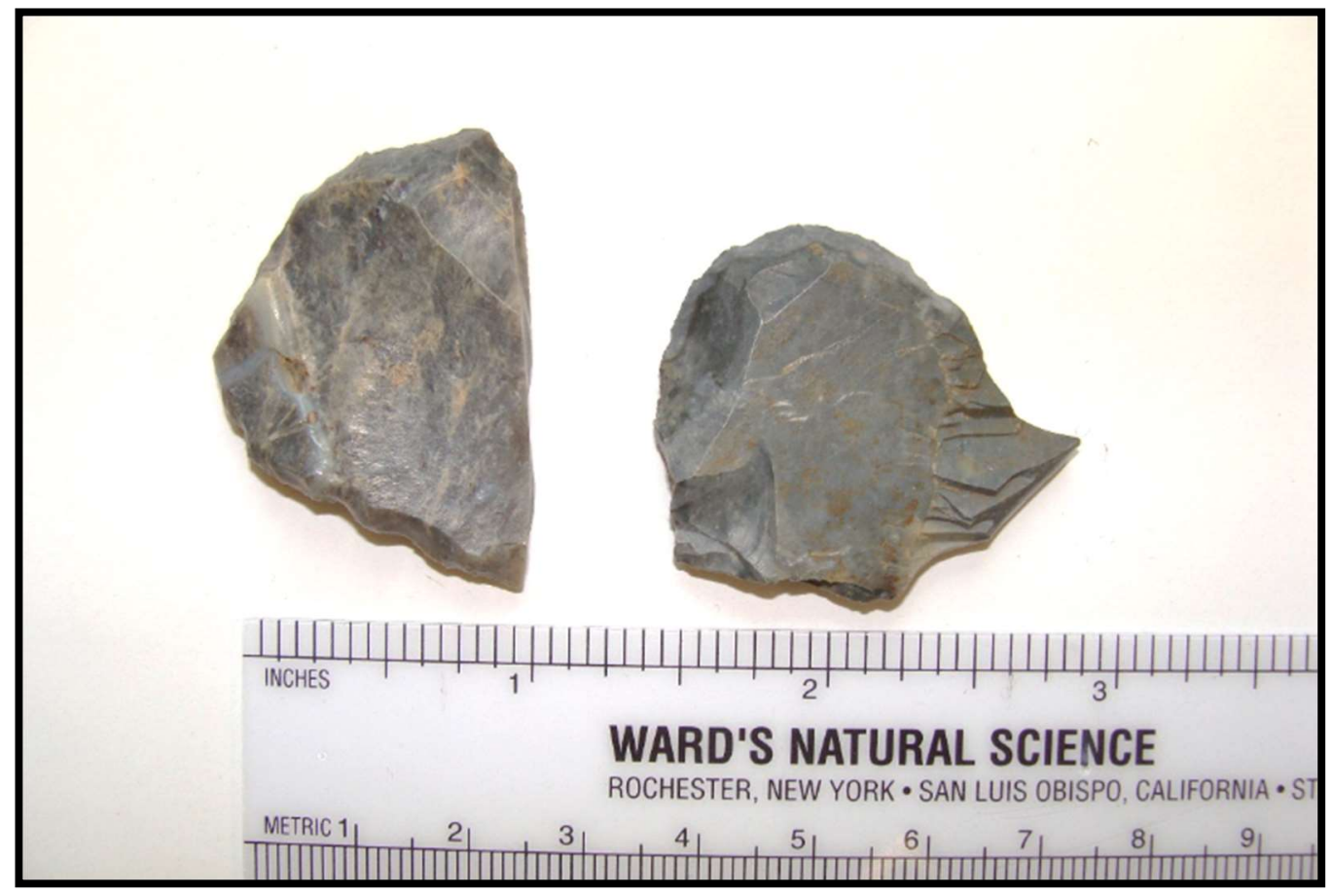

Figure S9. Left: Upper Mercer end scraper fragment (cat. \# 36). Right: Upper Mercer end scraper with possible burin break (cat. \# 34). Both recovered from Site 33AD30.

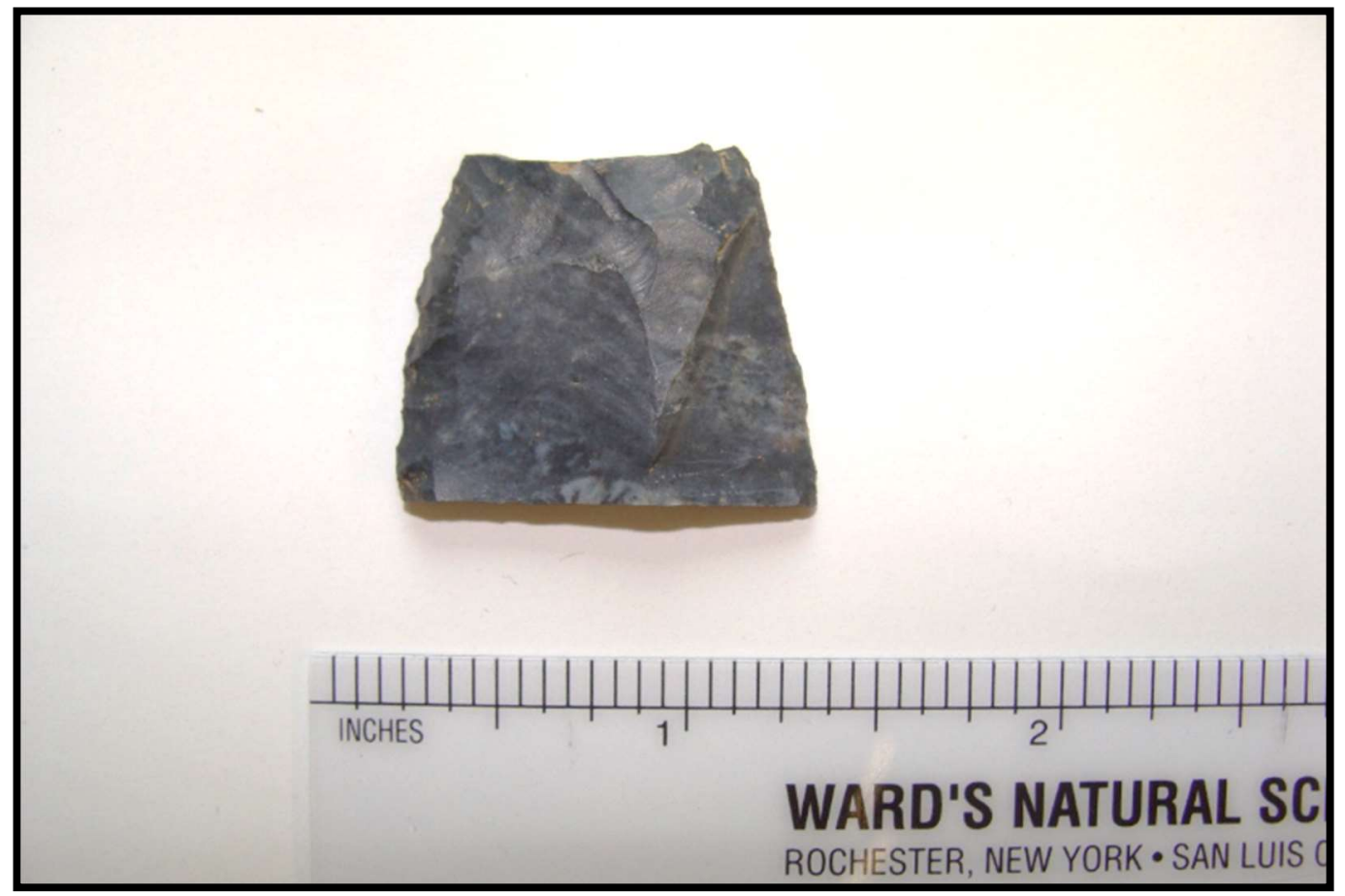

Figure S10. Upper Mercer indeterminate uniface fragment (cat. \# 35). "Flute-like" flake scar on outer surface and reworked into uniface. Recovered from Site 33AD30. 


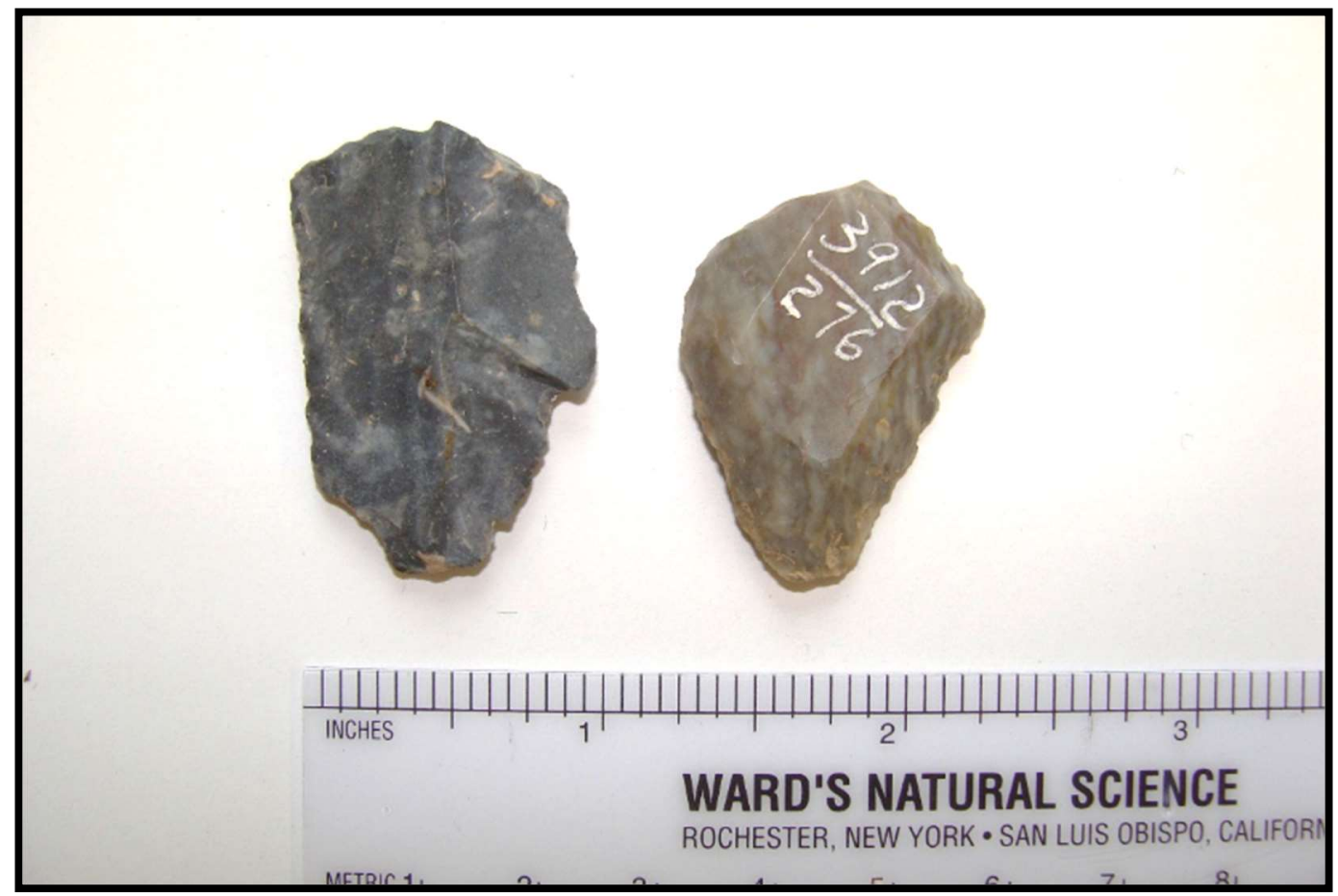

Figure S11. Left: Upper Mercer end scraper with graver (cat. \# 404) recovered from Site 33AD91. Right: Spurred end scraper of unknown fine-grained material (cat. \# 276) recovered from Site 33AD88.
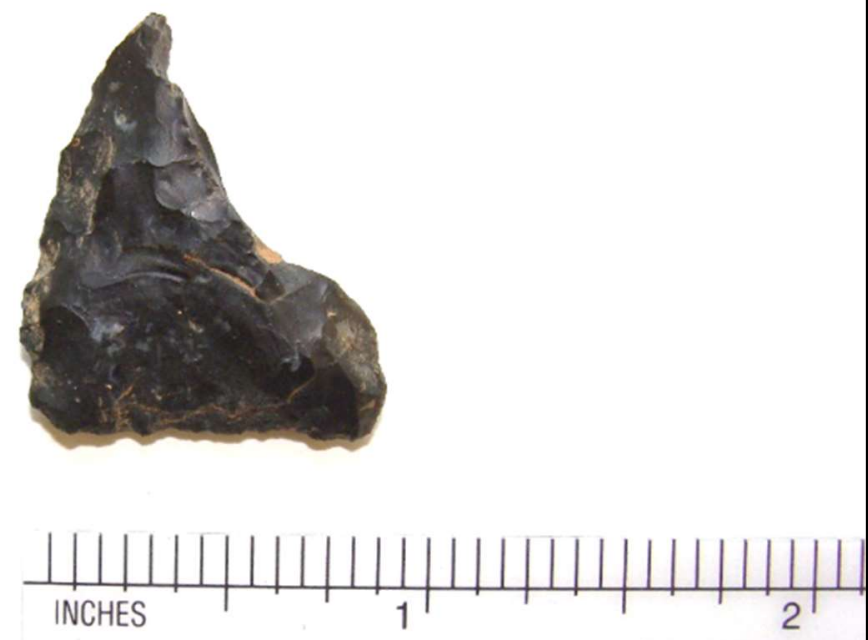

WARD'S NAT

ROCHESTER, NEW YOF

METRIC 1

$2+3$

5

Figure S12. Upper Mercer indeterminate biface fragment (cat. \# 286) recovered from Site 33AD88. Possible recycled fluted point fragment. 


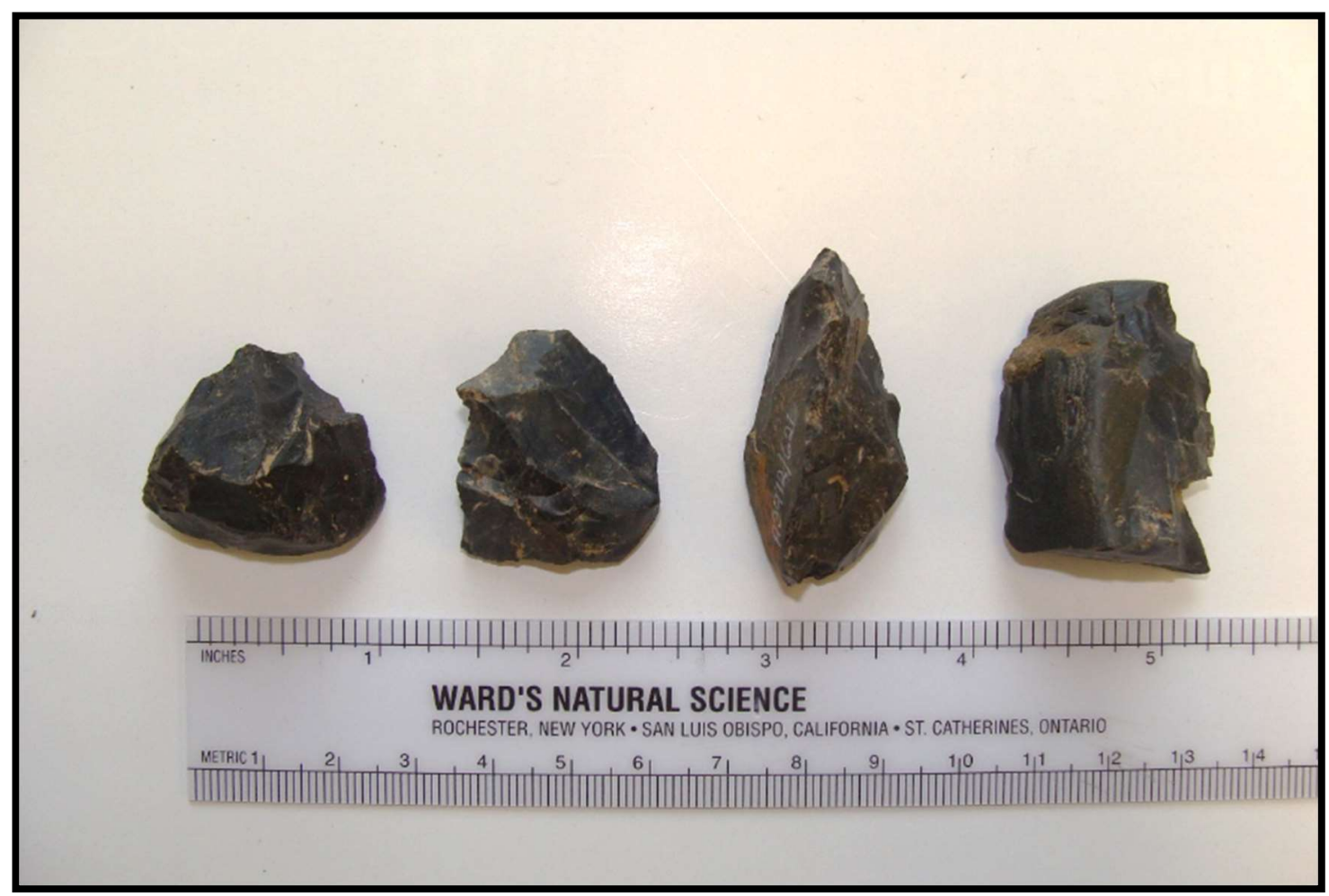

Figure S13. Upper Mercer cores (cat. \# 621) recovered from Site 33AD98. 


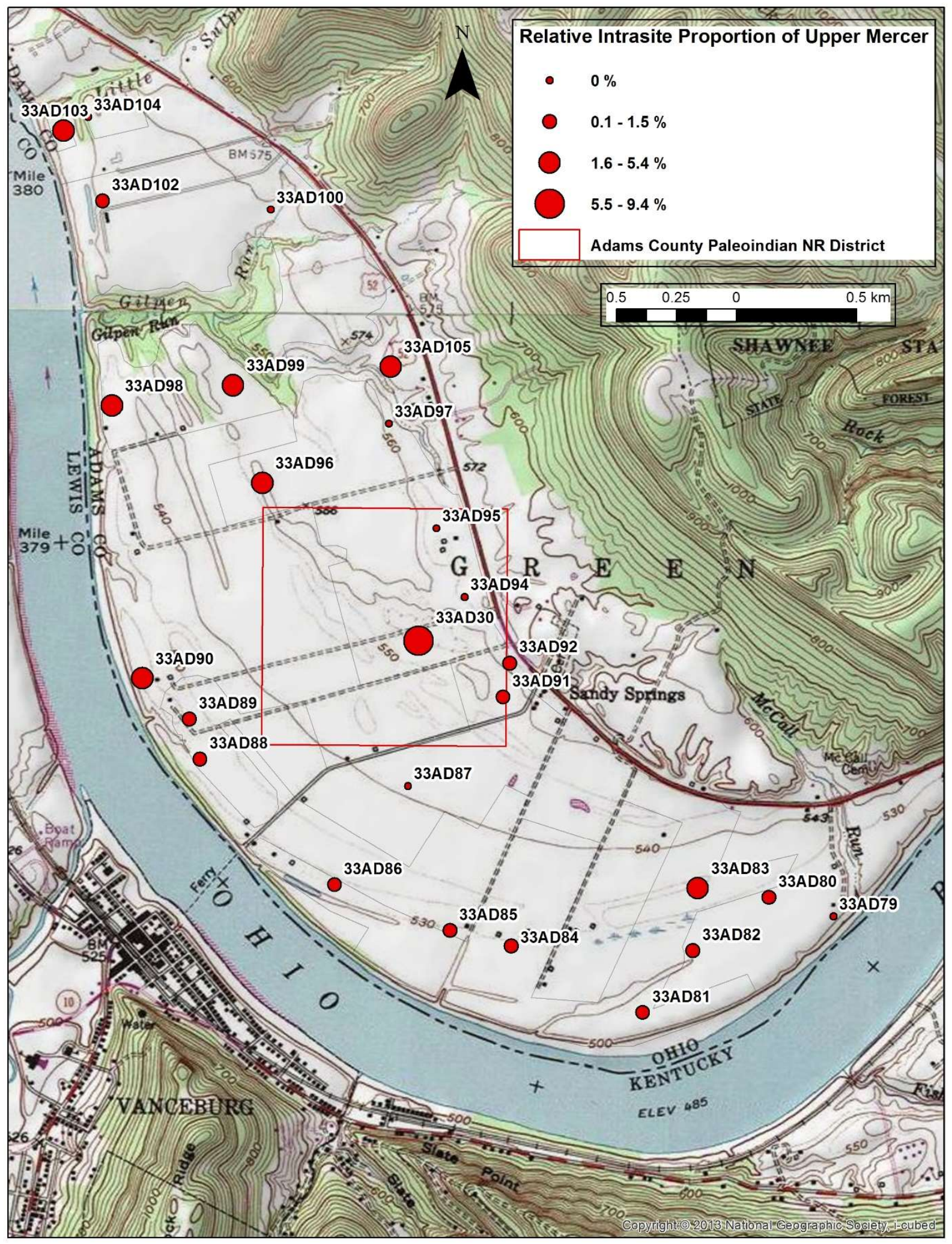

Figure S14. Graduated symbol map showing relative proportion of artifacts recovered during 1976 manufactured from Upper Mercer chert. Based on site centerpoints. 


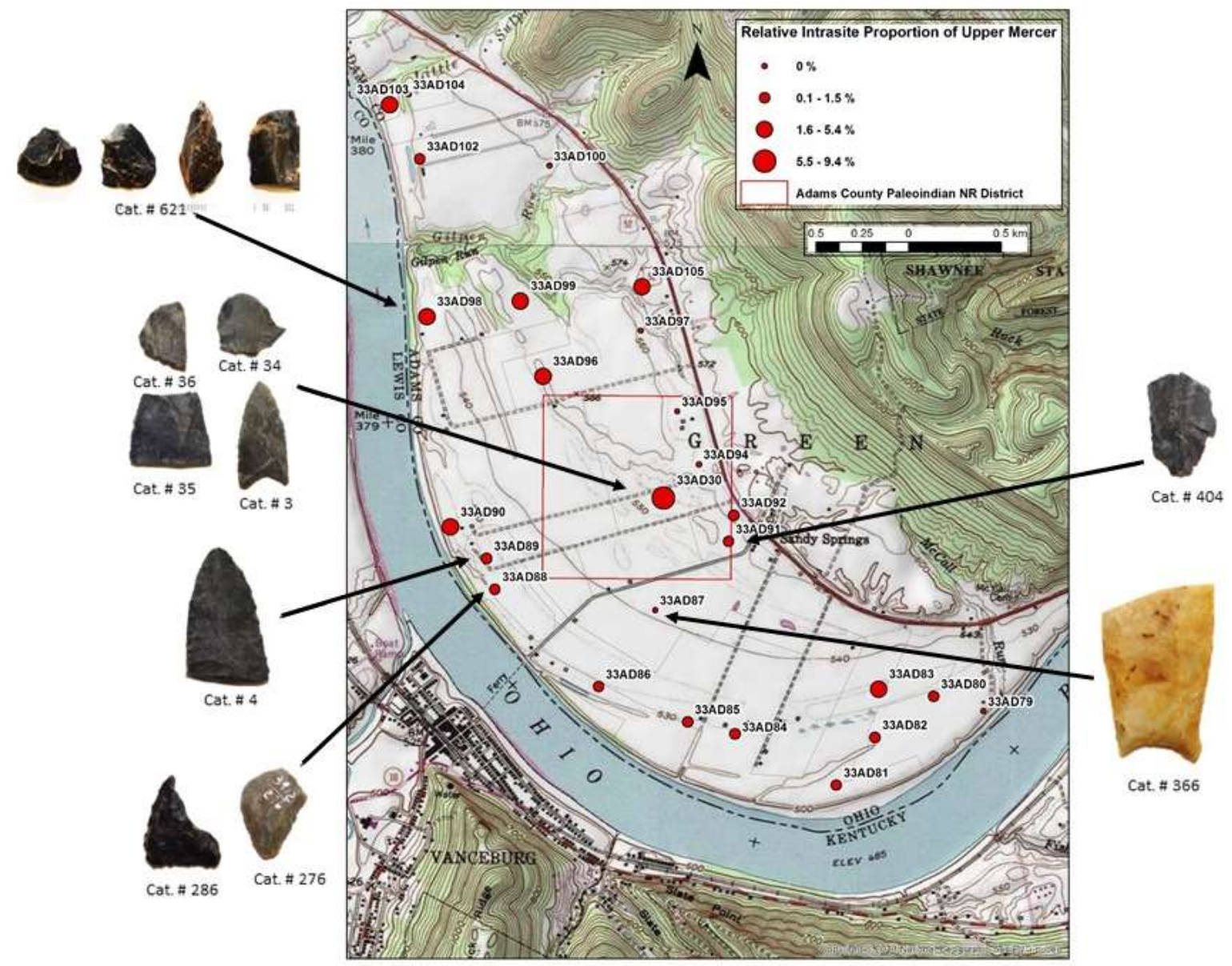

Figure S15. Plotting of suspected Paleoindian artifacts against backdrop of Upper Mercer chert distributions. 


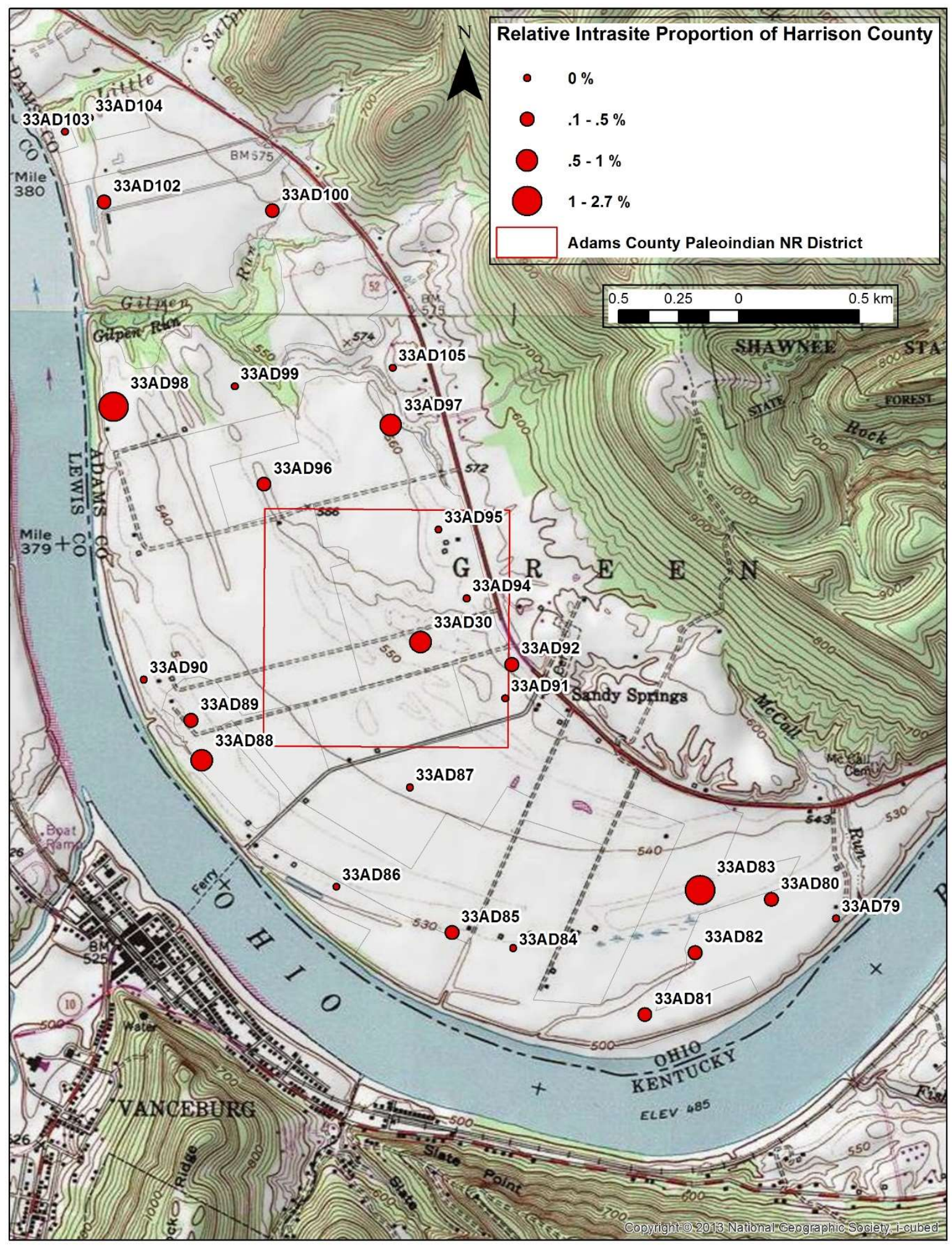

Figure S16. Graduated symbol map showing relative proportion of artifacts recovered during 1976 manufactured from Harrison County chert. Based on site centerpoints 


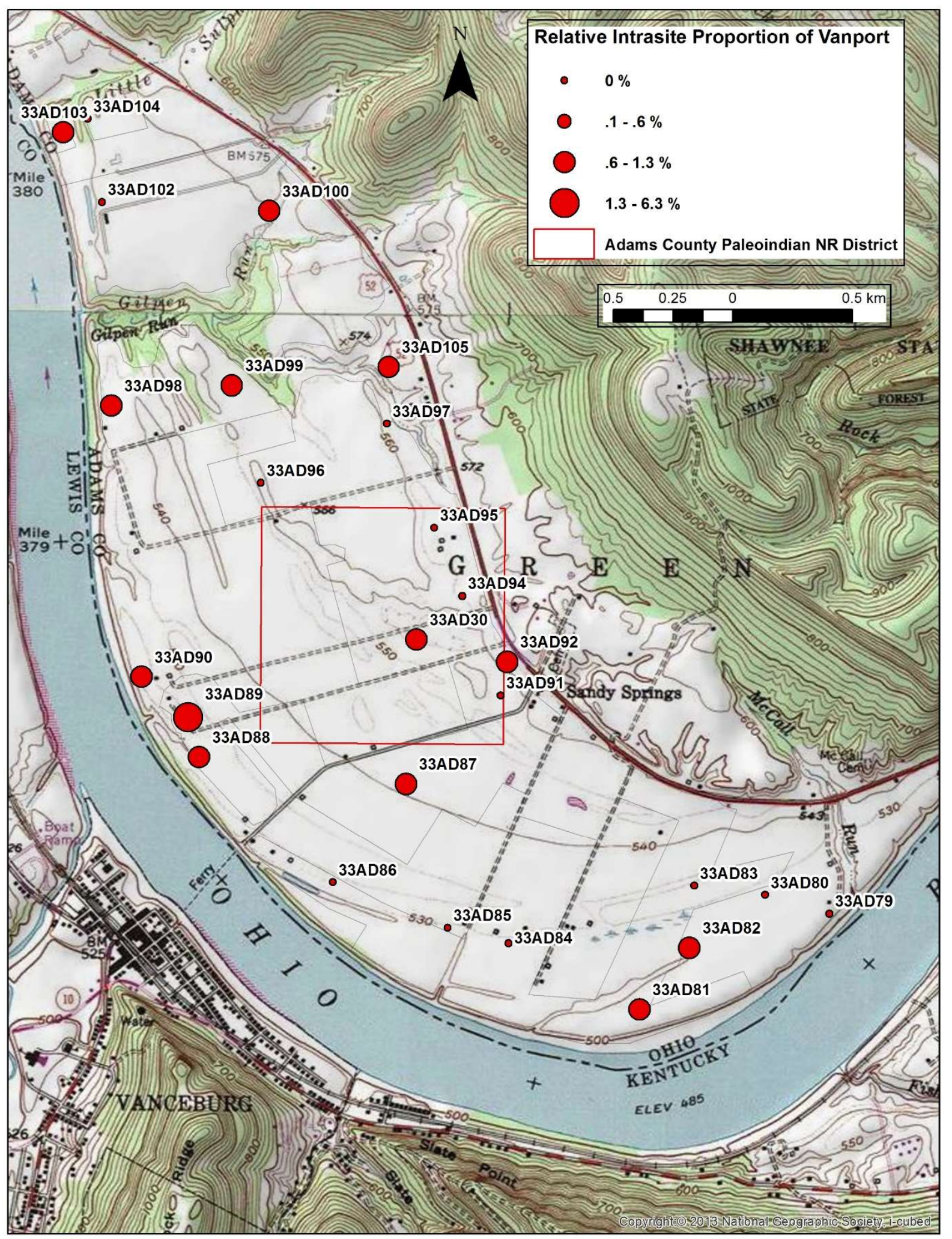

Figure S17. Graduated symbol map showing relative proportion of artifacts recovered during 1976 manufactured from Vanport chert. Based on site centerpoints. 


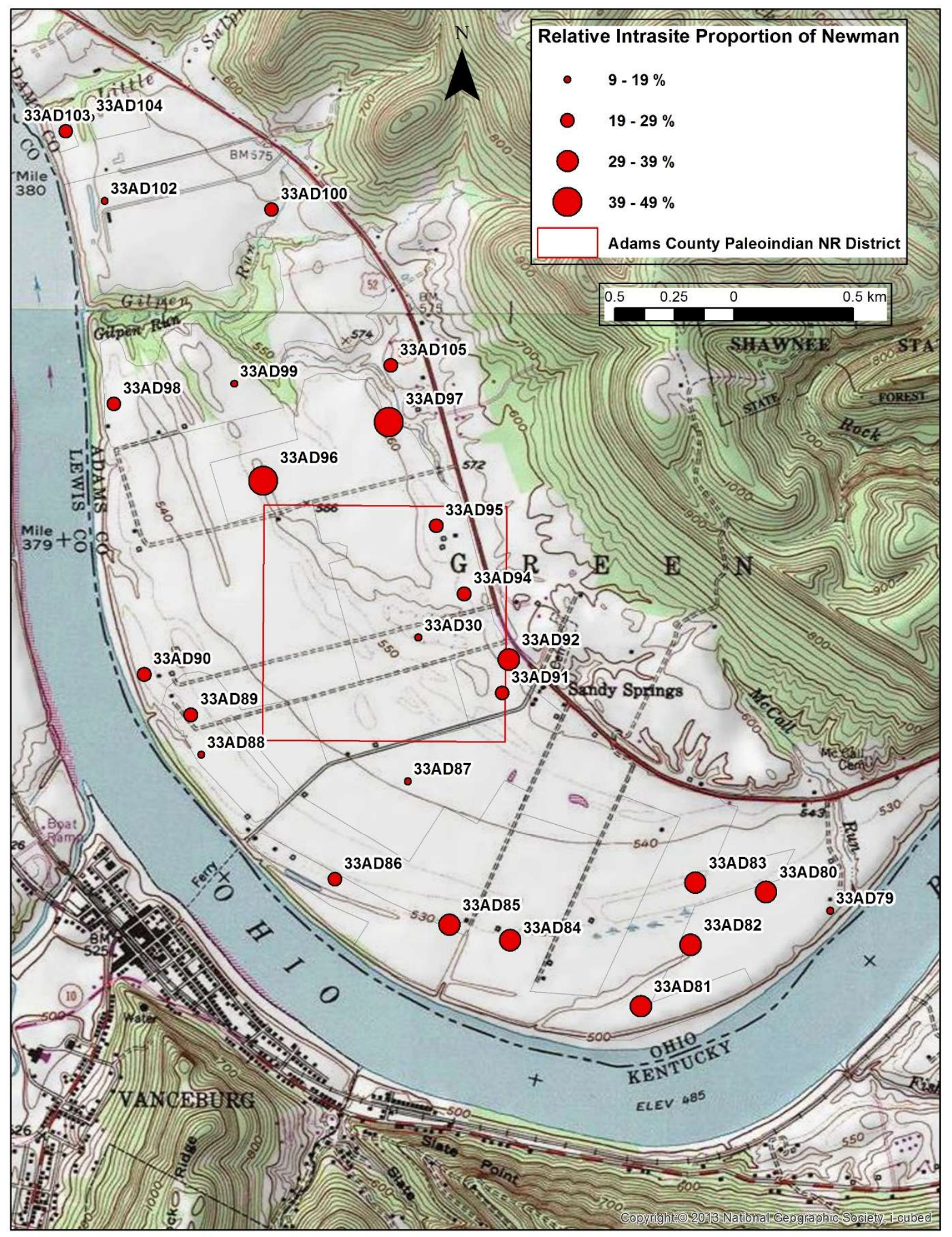

Figure S18. Graduated symbol map showing relative proportion of artifacts recovered during 1976 manufactured from Newman chert. Based on site centerpoints. 


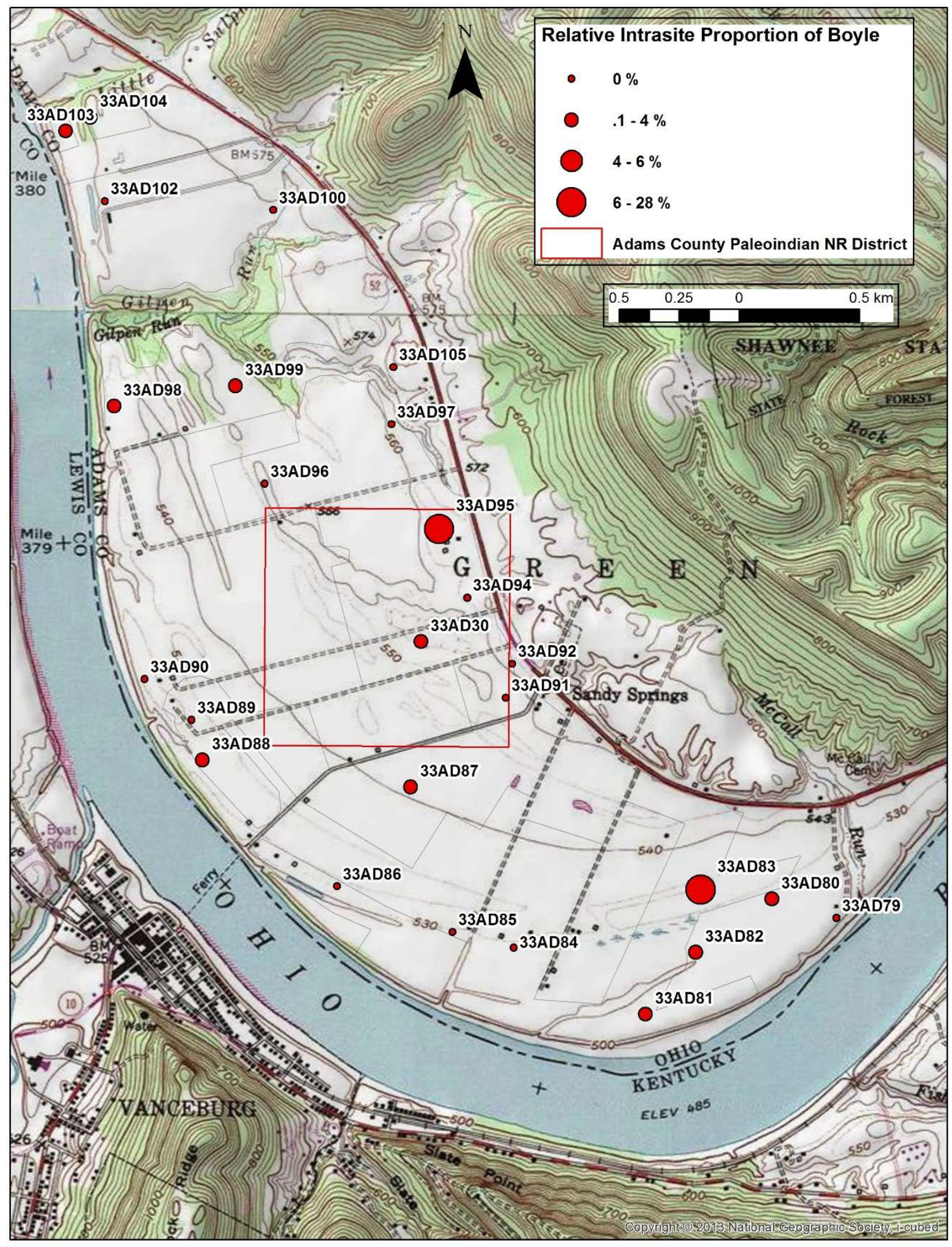

Figure S19. Graduated symbol map showing relative proportion of artifacts recovered during 1976 manufactured from Boyle chert. Based on site centerpoints. 


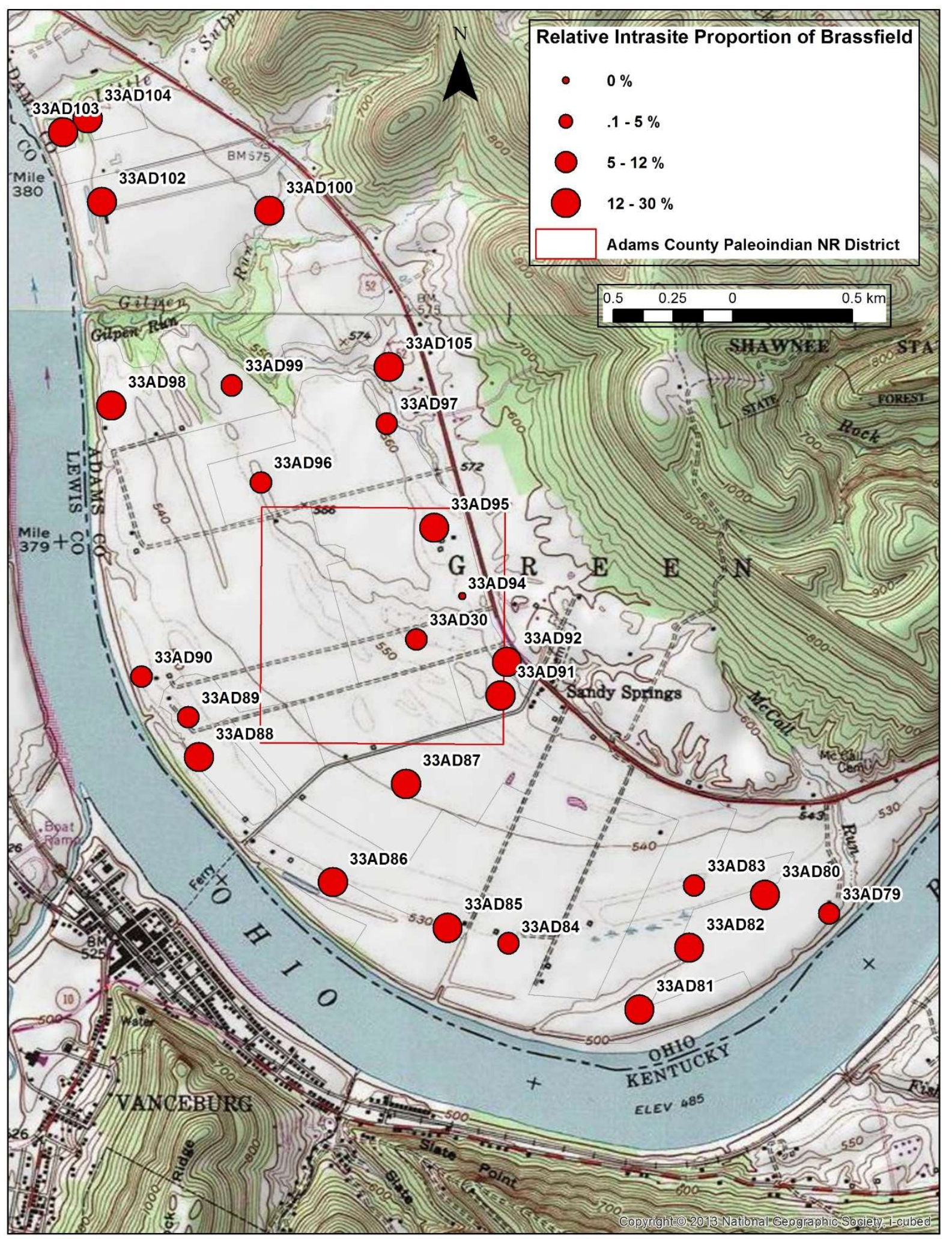

Figure S20. Graduated symbol map showing relative proportion of artifacts recovered during 1976 manufactured from Brassfield chert. Based on site centerpoints. 


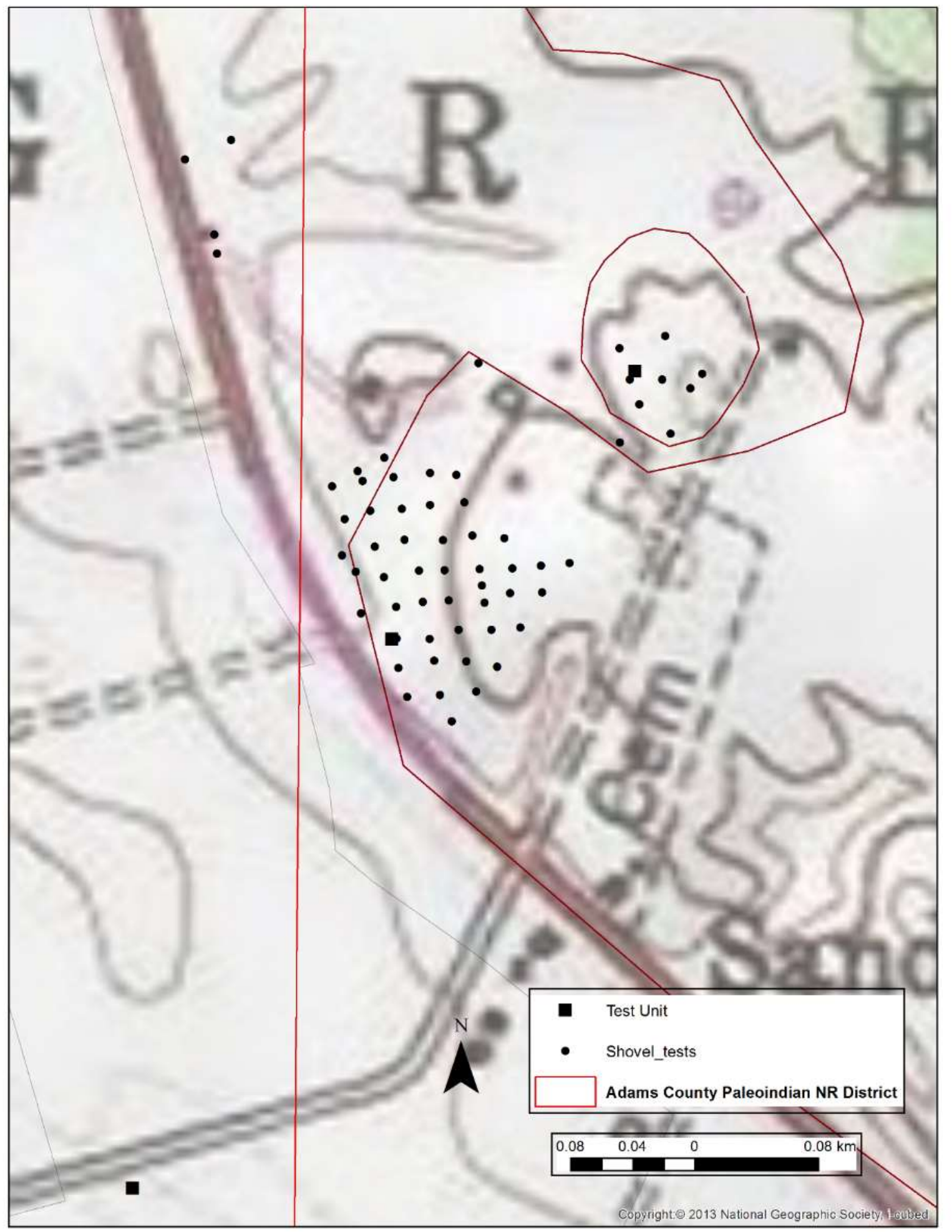

Figure S21. Location of shovel tests and test units excavated during the 2015-16. Location of Sandy Springs cemetery illustrated on background map. 


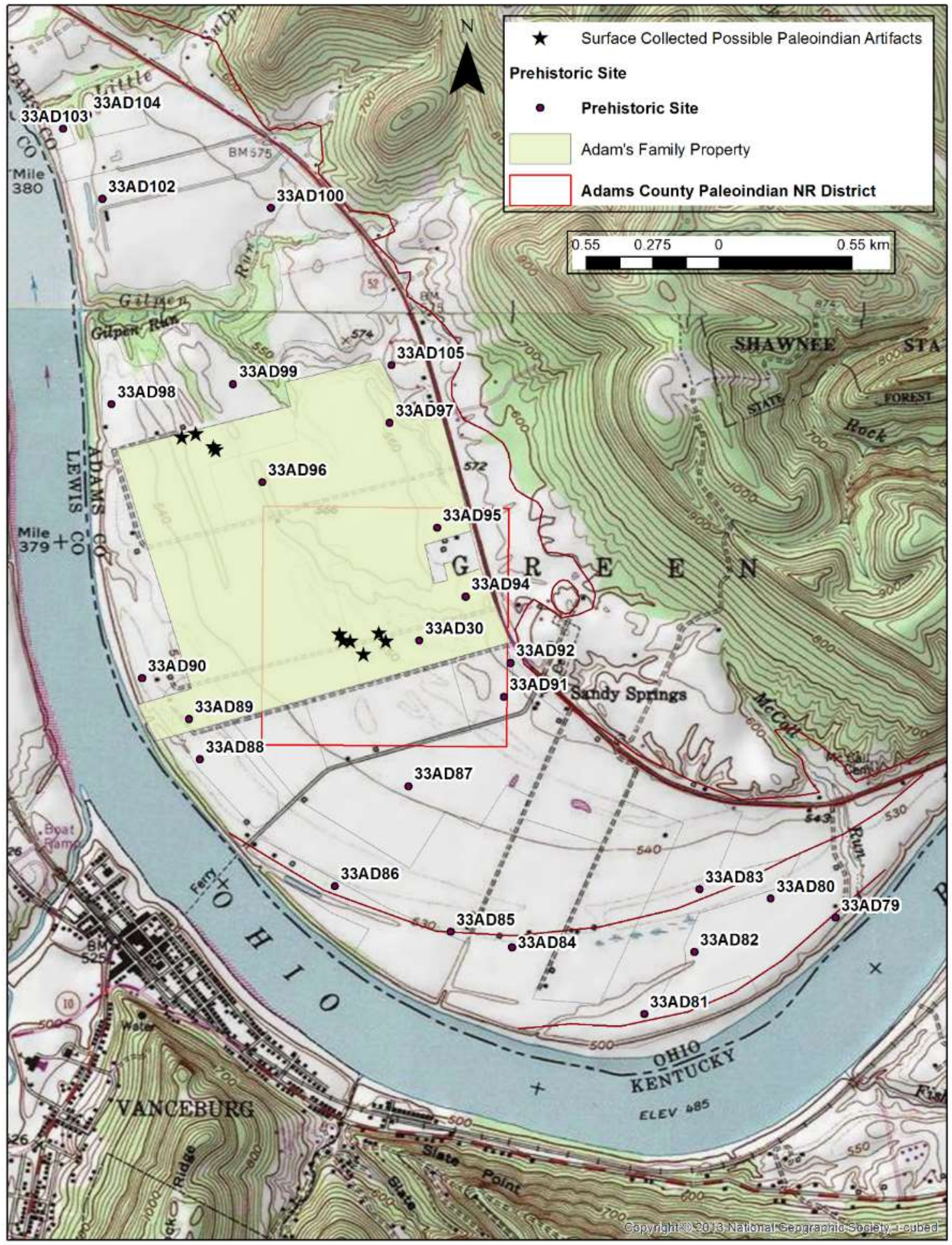

Figure S22. Location of potential Paleoindian artifacts recovered during surface collection of Adam's family property in 2015-16. Please note that the entire property was not subject to surface collection due to time constraints. 
CHAPTER 4. MIDWESTERN SAND DUNES, GEOARCHAEOLOGY, AND LIDAR: GEOMORPHIC LANDFORM ANALYSIS OF THE SANDY SPRINGS PALEOINDIAN SITE IN THE UPPER OHIO RIVER VALLEY

\section{Chapter 4. Introduction and Summary of Findings}

Development of a geochronology for the Sandy Springs landscape was proposed to better understand the potential spatial and stratigraphic distribution of Paleoindian material in the area (Purtill, 2015). In addition, this chapter seeks to determine the likelihood that aeolian sediments associated with relict dunes blanket unknown Paleoindian components on adjacent landforms. Such sedimentation is thought to have occurred through dune reactivation and mobilization events or through deposition of fines winnowed from nearby destabilized landforms. A manuscript of project results has been prepared and will be submitted to Geomorphology for consideration for publication. The primary findings of this research were as follows:

- Research indicates that Sandy Springs is located on a late Quaternary landscape shaped by aeolian-alluvial processes which have deposited sediments that have the potential to cover archaeological deposits including Paleoindian components.

- Aeolian-alluvial landforms that date to the late Pleistocene through Holocene have the potential to cover currently undocumented archaeological deposits including Paleoindian components.

- The broader impacts demonstrate that upper Ohio Valley Pleistocene landforms are commonly capped by late Pleistocene through Holocene aeolian sediments, a condition not always appreciated by archaeologists. This understanding significantly expands the inventory of landforms in the upper Ohio Valley that may contain buried archaeological components.

- A multi-tiered statistical and graphical approach to particle-size analysis is successful at discriminating depositional environments. This approach has great promise for developing geochronologies of late Quaternary landscapes and for determining the potential for buried archaeological components.

- Research also suggests that paleoclimate, including the well-documented 8.2 and $4.2 \mathrm{ka}$ events, may have played a significant role in shaping landscapes in the upper Ohio Valley throughout the late Pleistocene and the Holocene.

In addition to the main article text, this research produces additional information that can be developed into a future article. Micromorphological analysis is used to help determine the presence of clay aggregates in dune landforms that likely are the source of clay particles that form lamellae banding common in several dunes. 
Chapter 4. Journal Article Manuscript

Status: under review with Geomorphology

Late Quaternary aeolian and fluvial interaction in the upper Ohio Valley: The evolution of the Sandy Springs landscape, Adams County, Ohio (U.S.A.)

Authors:

Purtill, Matthew P.

Corresponding author:

Ball State University

Applied Anthropology Laboratories

Burkhardt Building (BB), room 314

Ball State University

Muncie, IN 47306

mpurtill@bsu.edu

Kite, J. Steven

West Virginia University

Department of Geology \& Geography

330 Brooks Hall, 98 Beechurst Ave.

Morgantown, WV 26506

jkite@wvu.edu

Forman, Steven L.

Baylor University

Department of Geology

Baylor University

One Bear Place \#97354

Waco, TX 76798

Steven_Forman@baylor.edu

Keywords:

Quaternary

Paleoclimatology

8.2 and 4.2 ka paleoclimate event

North America

Particle-size analysis

AMS and OSL dating 


\begin{abstract}
Geomorphic, pedogenic, stratigraphic, and sedimentary data, and chronologic observations were used to reconstruct aeolian and alluvial landform histories in the upper Ohio River Valley at Sandy Springs, Adams County, Ohio. Sandy Springs contains three geomorphic surfaces (S1-S3) that gradually rise between 6 and $46 \mathrm{~m}$ in elevation above a modern floodplain (S0). On higher S2 and S3 surfaces, sand- and silt-dominated lithostratigraphic units form complex and compound dunes, sand-mantled alluvial ridges, a longitudinal dune, a compound barchan-like dune, a climbing dune, an interdune sand sheet, and fine-textured coversands. Two aeolian facies (aeolian units I and II), four alluvial facies (alluvial units I, II, III, and IV), and one colluvial facies (colluvial unit I) were defined through a multi-tiered approach including consideration of principal component analysis of particle-size data, inspection of particle-size cumulative graphs, geomorphic context, eight optically stimulated luminescence ages, and a single radiocarbon age. Results indicate a late Quaternary landscape shaped by interaction between fluvial and aeolian processes since at least ca. $17 \mathrm{ka}$. Aeolian sedimentation was notably active between 11 and 1.4 ka on older S2 and S3 surfaces and is interpreted to be influenced by local wind fetch, sediment availability, and potential paleoclimate linkages. S3 coversand deposition at 8.2 ka may link to the North Atlantic Bond 5 paleoclimate event; whereas widespread dune reactivation and erosion at $4.5 \mathrm{ka}$ may represent prolonged drought previously documented for the Northern Hemisphere between 4.4 and $4.2 \mathrm{ka}$. Collectively, this study demonstrates that aeolian sedimentation actively sculpted many landforms in the upper Ohio Valley throughout the late Pleistocene and into the Holocene.
\end{abstract}

\title{
1.0 Introduction
}

Alluvial valleys are inherently dynamic systems shaped by a complex interplay of aeolian, fluvial, lacustrine, and glacial processes through time and space. Such interactions abound for the Ohio River in the eastern U.S. as it traverses multiple physiographic provinces of varied geological history including the Appalachian Plateaus, Interior Low Plateaus, Central Lowlands, and Coastal Plain (Fenneman \& Johnson, 1946). Although the timing and nature of fluvial, lacustrine, and glacial processes in the Ohio Valley have been studied to some degree (Counts, Murari, Owen, Mahan, \& Greenan, 2015; Fowke, 1925; Gray, 1984; Purtill, 2012; L. L. Ray, 1974; R. W. Robinson, 2009; Simard, 1989; Stafford, 2004; Stafford \& Creasman, 2002), research on aeolian landforms has focused on their sand-and-gravel mining potential (G. S. G. S. Fraser \& Fishbaugh, 1986; Friberg, 1970; Simard, 1989).

The association between aeolian landforms and proxies for Quaternary paleoclimate and paleowind reconstruction has yet to be defined rigorously in the Ohio Valley, as has been done for other aeolian depositional sequences (Arbogast, Luehmann, William Monaghan, Lovis, \& Wang, 2017; Booth et al., 2005; Forman, 2015; Forman et al., 2005; Hanson, Arbogast, Johnson, Joeckel, \& Young, 2010; Klemsdal, 2010; Nicholas Lancaster, 1981; J. A. Mason et al., 2011; J. A. Mason, Swinehart, Goble, \& Loope, 2004; Roskin, Tsoar, Porat, \& Blumberg, 2011; Sitzia et al., 2017; A. E. C. Stone \& Thomas, 2008; Telfer \& Thomas, 2007; Thomas, 2013; Wells, 1983; Wright, Forman, Waters, \& Ravesloot, 2011). In the eastern U.S., where relict sand dunes in alluvial settings are common, geomorphic research is revealing complex chronostratigraphies of 
aeolian and fluvial activity, some of which appear to be in response to late Pleistocene and Holocene droughts (Zoran Kilibarda \& Blockland, 2011; Krieg, Bettis, \& Forman, 2004; Miao, Hanson, Wang, \& Young, 2010; H Wang et al., 2011; Hong Wang, Stumpf, Miao, \& Lowell, 2012). Establishment of detailed chronostratigraphies are essential for accurate reconstruction of alluvial landscape histories and determination of valley sensitivity to past climatic conditions.

To better understand the depositional history of upper Ohio Valley aeolian and alluvial landforms, this paper presents geomorphic, pedogenic, stratigraphic, and sedimentary observations, and chronologic data for Sandy Springs, Adams County, Ohio (Fig. 4-1). Sandy Springs is a rolling, stepped-surface landscape characterized by sandy to silty aeolian landforms that straddle both the Ohio and Kentucky sides of the Ohio River (Chaplin \& Mason, 1967; Morris \& Pierce, 1967; Purtill, 2016; Purtill \& Kite, 2015). Sandy Springs also is known to archaeologists due to the presence of a substantial late Pleistocene Paleoindian archaeological site (33AD30), remains of which have been documented amongst the relict dunes (Cunningham, 1973; Purtill, 2017; Seeman et al., 1994). The archaeological site is listed to the National Register of Historic Places and appears associated with an early trail system connecting the upper Ohio Valley with parts of the Midsouth (Purtill, 2017). This research evaluates previous geological and pedological studies of Sandy Springs and proposes new interpretations regarding the depositional environments that formed geomorphic surfaces and associated aeolian landforms. Furthermore, this study considers possible links between paleoclimate events and the development of aeolian landforms at Sandy Springs. Particle-size data, principal components analysis (PCA), particle-size cumulative graphs, optically stimulated luminescence (OSL) and accelerator mass spectrometry (AMS) radiocarbon ages, and geomorphic context are used to define two aeolian facies (aeolian units I and II), four alluvial facies (alluvial units I, II, III, and

IV), and one colluvial facies (colluvial unit I). This late Quaternary landscape, constrained by the rolling alluvial topography, reflects a complex interaction of fluvial and aeolian processes since at least ca. $17 \mathrm{ka}$. OSL ages suggests substantial aeolian sedimentation occurred at Sandy Springs throughout the late Pleistocene and Holocene; some of may be linked with previously documented paleoclimate episodes at $8.2 \mathrm{ka}$ and 4.4 to $4.2 \mathrm{ka}$ (Booth et al., 2005; Y.-X. Li, Yu, \& Kodama, 2007; Willard, Bernhardt, Korejwo, \& Meyers, 2005).

\subsection{Environmental Background}

Sandy Springs is located in a heavily dissected section of the Shawnee-Mississippian Plateau of the Appalachian Plateaus province (Brockman, 2006). Regional bedrock geology includes Silurian, Devonian, and Mississippian shales, limestones, siltstones, and thickly bedded sandstones (Coogan, 1996; Slucher et al., 2006). The upper Ohio River flows within a broad valley bottom, averaging $2.3 \mathrm{~km}$ in width, between the Scioto River confluence at Portsmouth, Ohio, and Sandy Springs. Below Portsmouth, the Ohio River has a straight channel pattern $($ sinuosity $=1.09)$ for ca. $19 \mathrm{~km}$ until it reaches Garrison, Kentucky, at which point the river becomes more sinuous (sinuosity $=1.16$ ) through Sandy Springs (Fig. $4-1)$. The $\sim 14 \mathrm{~km}$ reach between Garrison and Sandy Springs represents the first major meander bend downriver from the Scioto River, a major Wisconsinan glacial outwash system (Hubbard, 1954; Kempton \& Goldthwait, 1959; L. L. Ray, 1974). Immediately downriver from Sandy Springs, where bedrock geology transitions from Devonian to Silurian rocks, the valley constricts to an average width of $1.5 \mathrm{~km}$ and again straightens for $\sim 22 \mathrm{~km}$ (sinuosity =1.08). Currently, pool elevation of this 
reach of the Ohio River is dam-controlled at $148 \mathrm{~m}$, although early navigation charts list a lowwater elevation of $141 \mathrm{~m}$ at Sandy Springs (Jones, 1916: 163). Low-water elevations are used in this study to approximate pre-dam pool averages (following Simard, 1989: 21-25). The highwater mark for the January 1937 flood is $164 \mathrm{~m}$ at Sandy Springs (Morris \& Pierce, 1967), which is the flood of record for the Ohio River (NOAA, 2017).

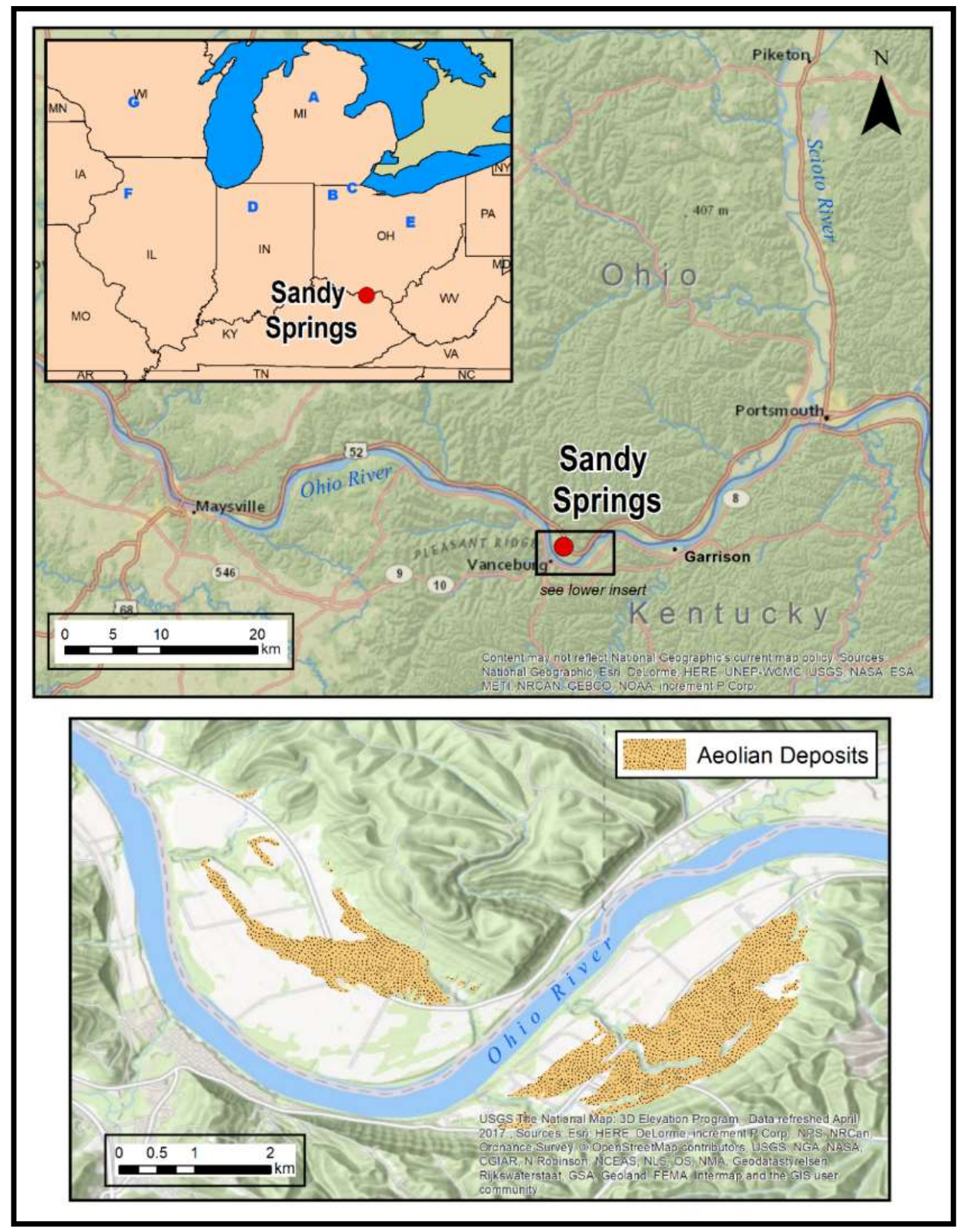

Fig. 4-1. Upper image: location of Sandy Springs along the Ohio River and additional sites mentioned in the text (A=Arbogast et al., 2015; B = Blockland 2013; $\mathrm{C}=$ Campbell et al., 2011; D $=$ Kilibarda and Blockland 2011; $\mathrm{E}=$ Lutz et al., 2007; $\mathrm{F}=$ Miao et al., 2010; $\mathrm{G}=$ Rawling et al., 
2008). Lower image: combined distribution of sediments interpreted as aeolian in geologic and pedologic literature (Chaplin \& Mason, 1967; Morris \& Pierce, 1967; USDA-NRCS, 2017). This study focuses on area north of the Ohio River.

Sandy Springs has a broad stepped-surface landscape that gently slopes towards the Ohio River. Previous work defined as many as four alluvial/outwash surfaces or terraces above a modern floodplain (Morris \& Pierce, 1967; Pavey et al., 1999). Morris \& Pierce (1967) mapped a distinct terrace at $185 \mathrm{~m}$ of supposed Illinoian age (ca. $160 \mathrm{ka}$ ) and three surfaces at 175, 169, and $166 \mathrm{~m}$ of Wisconsinan age, sensus lato; 70 to $15 \mathrm{ka}$ ago (Morris \& Pierce, 1967). These surfaces are underlain by up to $\sim 45 \mathrm{~m}$ of well-sorted, commonly cross-bedded gravels, sands, silts, and clay. The highest Illinoian landform is mantled with up to $5 \mathrm{~m}$ of silty sediment interpreted as loess (Morris \& Pierce, 1967).

In contrast, later mapping at Sandy Springs identified only two outwash landforms (O1, O2) above modern alluvium (Pavey et al., 1999). A high wedge-shaped O1 landform composed of gravel and sand appears to be an outwash terrace constructed during the Late Wisconsinan, ca. 22 to $18 \mathrm{ka}$ (Pavey et al., 1999), which is markedly younger than Illinoian age reported by Morris and Pierce (1967). The lower expansive O2 landform is composed of gravel and sand outwash with an inferred age of 18 to $15 \mathrm{ka}$ and appears correlative to Morris and Pierce's (1967) Lower, Middle, and Upper Wisconsinan terraces.

Several soil series are mapped at Sandy Springs (Lucht \& Brown, 1994). Lower to middle elevation surfaces primarily are mapped on well drained landforms as Elkinsville silt loam (Ultic Hapludalfs) and Sciotoville silt loam (Aquic Fragiudalfs), and on poorly drained landforms as Peoga silt loam (Fragic Epiaqualfs). Sandy soils on these surfaces are mapped as Plainfield sand (Typic Udipsamment) in Ohio and Lakin loamy sand (Lamellic Udipsamments) in Kentucky (USDA-NRCS, 2017). The high surface (O1 following Pavey et al., 1999) is mapped as Otwell silt loam (Oxyaquic Fragiudalfs). Plainfield and Lakin series soils form on aeolian landforms that exhibit complex morphologies identified at Sandy Springs as dunes. Dunes are restricted to midelevation surfaces and cover ca. 450 ha of land (Morris \& Pierce, 1967; Purtill, 2017; Purtill \& Kite, 2015).

Pollen data from contexts in the upper Ohio Valley (Fredlund, 1989; Purtill, 2012: 42-47) indicate a transition in arboreal composition during the late Pleistocene to mid-Holocene. Data indicate an early savanna-like setting dominated by spruce (Picea sp.) and grasses during the late Pleistocene transitioned by the mid-Holocene into a mixed mesophytic forest of oak (Quercus sp.), hickory (Carya sp.), maple (Acer sp.), chestnut (Castanea sp.), walnut (Juglans sp.), and elm (Ulmus sp.).

Remnant sand-prairie vegetation grows on sandy soils not under current cultivation. Where preserved, the prairie supports xeric plant species, including eastern prickly pear cactus (Opuntia humifusa), little whitlow grass (Draba brachycarpa), passion flower (Passiflora incarnata), silkgrass (Chrysopis graminifolia), and spreading sandwort (Arenaria patula) (Purtill, 2017; M. A. Vincent et al., 2011). 
The modern climate for southern Ohio is temperate with hot summers but no significant precipitation shortages throughout the year (Peel et al., 2007; Thornthwaite, 1931). Average annual precipitation is $1092 \mathrm{~mm}$, with the highest monthly totals between March and August, while mean annual temperature is $11.8^{\circ} \mathrm{C}$ (Lucht \& Brown, 1994: 124). Modern surface wind speed and direction vary seasonally. Based on 1930-1996 climatic data from stations at Lexington and Jackson in Kentucky; Cincinnati, Columbus, and Dayton in Ohio; Pittsburg, Pennsylvania; and Huntington, West Virginia; surface wind direction at Sandy Springs ranges from S to WSW with more southerly orientation during summer months and a more western orientation during winter months (National Climatic Data Center, 1996). Paleoclimatic reconstructions from late Pleistocene proxies suggest a more northerly (WNW) wind direction was typical in eastern North American during the Wisconsinan glaciation (Zoran Kilibarda \& Blockland, 2011; Thorson \& Schile, 1995; Wells, 1983).

\subsection{Methods}

\subsection{Sample locations, field and laboratory descriptions, and morphometric landform analysis}

To best capture the complex history of landscape evolution, geomorphic, pedogenic, sedimentary, and stratigraphic information were obtained through inspection of 33 profile sections and 203 sediment samples (see Supplementary Material, Appendix A, Fig. S23 and S22). Sections were dispersed across several geomorphic surfaces and settings, and included cutbank exposures (prefixed 'CB'), bucket augers ('BA'), soil test pits ('STP' or 'U'), and auger holes extracted by a truck-mounted $4.1-\mathrm{cm}$ diameter Giddings probe ('GP'). Sampling ranged from the modern surface to as deep as $4.5 \mathrm{~m}$. Sediment samples were described using NRCS-USDA standards for texture, structure, sorting, horizon assignment, and contacts (Schoeneberger, Wysocki, Benham, \& Staff, 2012). Sedimentary structures were described in the field and attention was given to bedding contacts and dip directions. Lithological discontinuities were inferred through combined field observation and laboratory identification of textural breaks, shown by sand fraction mean and standard deviation values, and uniformity values for the $<2 \mathrm{~mm}$ fractions (e.g., Cremeens and Mokma, 1986; Schaetzl, 1998; Schaetzl and Anderson, 2005: 224). Micromorphologic properties were examined in 12 thin sections from 7 geomorphic sections as a means to further explore, or confirm, sedimentologic and pedologic formation dynamics. Micromorphology analysis followed established protocols and terminology of FitzPatrick (1984), Stoops (2003, 2010), and Vepraskas \& Wilson (2008). Munsell values were determined in the laboratory on moistened samples using a Konica Minolta CR-400 Chroma Meter.

Airborne laser altimetry (LiDAR) data aided morphometric analysis of discrete landform elements (e.g., dunes, ridges, knobs, etc.) and helped characterize the continuous land surface (i.e., geomorphic surfaces) (Fig 2.). Data tiles at $0.762 \mathrm{~m}$ resolution in ESRI Grid format were obtained from the Ohio Geographically Referenced Information Program website (OGRIP, 2015). Sand dune classification was based on morphometric traits derived from LiDAR data and followed established classifications (Cooke, Warren, \& Goudie, 1993; Nick Lancaster, 2011; Pye, 1982; Thomas, 2011). In cases where sedimentary structures within dunes could be identified, initial dune classifications were verified. 


\subsection{OSL and AMS Dating}

The chronology presented here is based on age determinations on one AMS radiocarbon sample and eight OSL sediment samples. The radiocarbon age was obtained through Beta Analytic, Inc. on a sample from a buried A horizon (2Ab horizon) at $\sim 0.55 \mathrm{~m}$ depth in section CB4. The radiocarbon age was calibrated using IntCal13 software (Reimer et al., 2013). Eight sediment samples were retrieved from various geomorphic contexts and submitted for OSL dating (Aitken, 1998) at the Geoluminescence Dating Research Lab at Baylor University. Single aliquot regeneration (SAR) protocols (Murray \& Wintle, 2003) were used for OSL dating to estimate the apparent equivalent dose of the $63-44,250-355,355-425$, and $425-500 \mu \mathrm{m}$ quartz fractions for 28 to 61 separate aliquots. A full discussion of OSL methods is provided in Supplemental Material, Appendix B.

\subsection{Particle-size analysis}

Particle-size analysis was conducted via sieve-pipette method (Folk, 1974; Poppe, McMullen, Williams, \& Paskevich, 2014) using the Wentworth, or logarithmic phi $(\varphi)$, scale between $-1 \varphi$ and $10+\varphi$ in whole-step increments. This study divides clay and silt at $0.004 \mathrm{~mm}$ or at $8 \varphi$. Upon air drying and removal of macro-organics, 10-40 $\mathrm{g}$ of sediment from each sample were ground in a pestle and mortar and oven dried at $65^{\circ} \mathrm{C}$ for 24 hours to remove water weight. The larger $40 \mathrm{~g}$ samples were reserved for samples of high sand content following Twenhofel and Tyler (1941) to assure adequate silt-clay proportions for analysis. Next, $10 \mathrm{ml}$ of dispersing solution (10\% sodium hexametaphosphate) and $40 \mathrm{ml}$ of deionized water was added to the sample, agitated, and allowed to stand for 24 hours. The sample then was mechanically agitated for 15 minutes and wet sieved through $0.0625 \mathrm{~mm}$ mesh to segregate coarse from fine fractions. Upon drying, coarse sediment was dry sieved through six nested sieve screens ( $\varphi$ units $-1,0,1,2,3,4)$ via a Ro-tap mechanical shaker for 15 minutes then weighed. The fine fraction was subject to pipette withdraw on a timed basis following Poppe et al. (2014). Pipetted samples were oven dried, then weighed.

Statistics were calculated on grain size $\varphi$ distributions including graphical median $\left(M d_{\varphi}\right)$ (Folk \& Ward, 1957); first moment mean $\left(\bar{x}_{\varphi}\right)$, second moment standard deviation $\left(\sigma_{\varphi}\right)$, third moment skewness $(S k)$, fourth moment kurtosis $(K)$ (Friedman, 1961; Pye and Tsoar, 2009: 58-60); and coefficient of variation ( $C V$ ) (Wong and Lee, 2005: 65). $M d_{\varphi}, \bar{x}_{\varphi}, \sigma_{\varphi}, S k$, and $K$ statistics were calculated using Gradistat V.8 software (Blott \& Pye, 2001). The above-listed six textural parameters were calculated on three dataset iterations which resulted in a total of 18 separate statistics calculated as part of the PCA. First, six statistical parameters were calculated for the entire $\varphi$ distribution range $(-1 \varphi-10+\varphi)$. Second, to limit potential effects of pedogenic weathering or additions of dust, the same statistical parameters were recalculated on a clay-free basis (-1 $\varphi$ through $8 \varphi ; \varphi$ clay-free) (Ivester and Leigh, 2003: 302; Leigh, 1998: 314; Schaetzl and Anderson, 2005: 219). Finally, since the sand-sized grains are comparatively immobile in soil profiles (Karathanasis and Macneal, 1994; Schaetzl and Anderson, 2005: 219), the third iteration of statistics was calculated that included only sand fractions $(0 \varphi-4 \varphi ; \varphi$ sand $)$ as a means to better characterize primary depositional environments.

A PCA, a data reduction technique that transforms individual variables into orthogonal components (Field, 2009: 633-641; Rogerson, 2010: 297-302), was calculated in SPSS v. 18.0 to further explore the graphical and moment statistical results. Although PCA has not been used 
previously in particle-size analysis, at least to the author's knowledge, other data reduction and multivariate statistical approaches have been employed to interpret depositional environments including discriminant analysis (Kasper-Zubillaga \& Carranza-Edwards, 2005; Moiola \& Spencer, 1979; Moiola, Spencer, \& Weiser, 1974; Purkait \& Majumdar, 2014), factor analysis (Klovan, 1966), and logistic regression (P. Vincent, 1986). Sedimentary facies were defined for samples based on several criteria including PCA results, particle-size cumulative graphs, consideration of geomorphic context, and chronometric dates.
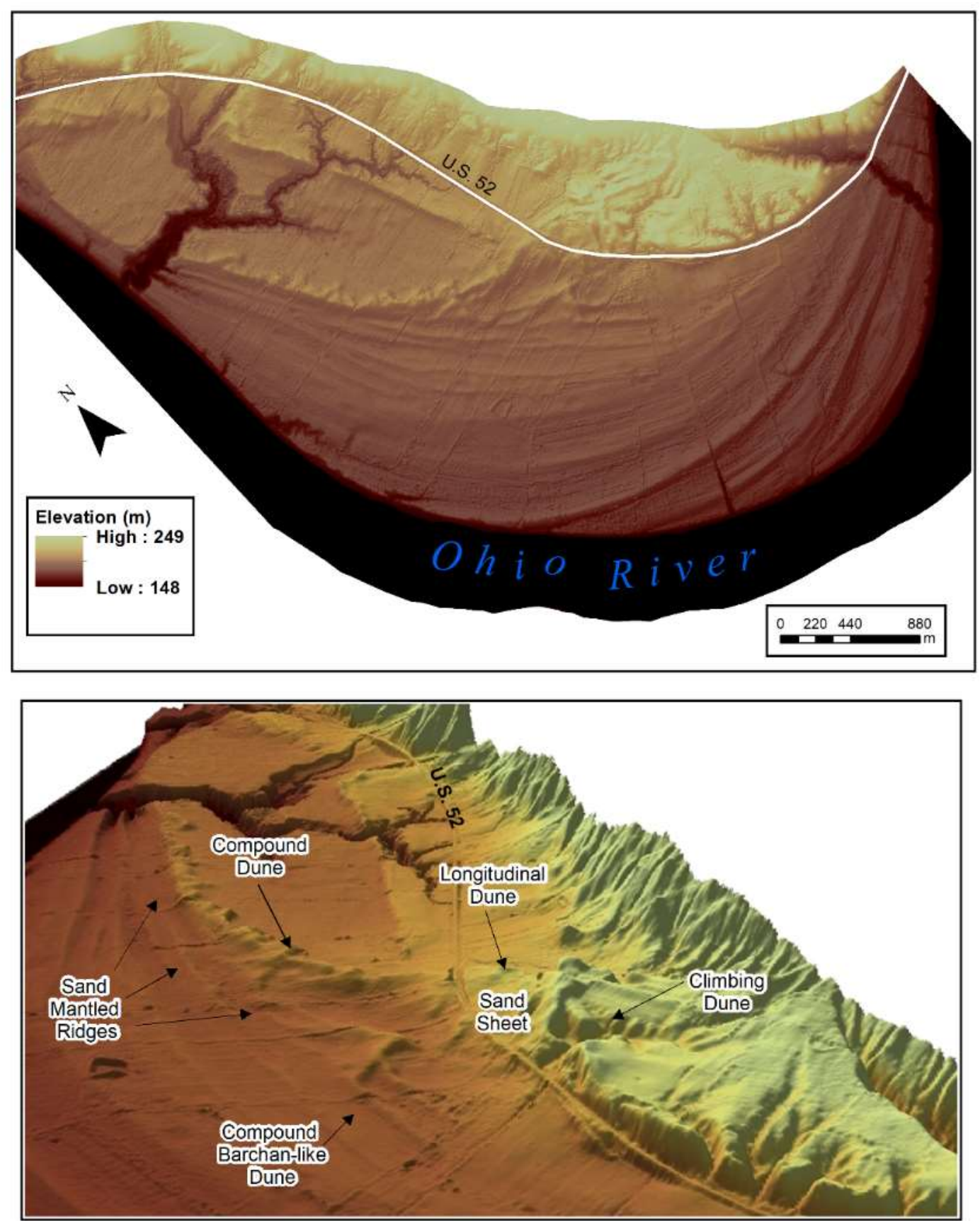

Fig. 4-2. Upper image: Side-illuminated vertical 2D LiDAR image showing the Sandy Springs landscape. Lower image: rotated view of upper image illustrated with ArcScene oblique 3D 
representation of LiDAR image at 3x vertical exaggeration showing dune types, interdunal sand sheet, and sand-mantled ridges.

\subsection{Results}

Building upon Morris and Pierce (1967) and Pavey et al. (1999), this study defined three geomorphic surfaces (S1-S3) above a modern floodplain (S0) (see also Purtill, 2016, 2017; Purtill \& Kite, 2015) (Fig. 4-3). Surfaces were defined following inspection of topographic and longitudinal profile data, chronometric dates, interpretation of DEM data using TerEx software (J. C. Stout \& Belmont, 2013), and review of a regional geoarchaeological assessment (e.g., Purtill, 2012: 21-41).

\subsection{SO and S1 Geomorphic Surfaces}

The modern floodplain (S0) is a narrow landform separated from S1 by a 6-m escarpment. The $\mathrm{S} 1$ tread rises 17 to $21 \mathrm{~m}$ above the Ohio River's pre-lock \& dam low-water elevation of $141 \mathrm{~m}$ (Jones, 1916: 163). The S1 landscape is characterized by pronounced ridge-and-swale topography with an azimuthal orientation close to true E to W. Although not directly dated during this study, the S1 may be correlative to a T2 surface $17 \mathrm{~m}$ above the low-water mark of 139 m downriver at Stuart Station (near Maysville, Ky., Fig. 4-1). If correlative, an AMS radiocarbon age of 10,600 +/- $50 \mathrm{BP}(12,696$ to 12,426 cal yrs BP; Beta 256762) from $2 \mathrm{~m}$ below the T2 surface at Stuart Station (Purtill, 2012: 28) indicates a predominately Holocene age for the uppermost S1 overbank fine sediments.

\subsection{S2 Geomorphic Surface, Sand Dunes, and Sand Mantles}

Rising from 21 to $39 \mathrm{~m}$ above the Ohio low-water elevation, the S2 surface gradually slopes $1.7 \%$ towards the Ohio River and has low ridge-and-swale topography. Azimuthal orientation of $\mathrm{S} 2$ ridge-and-swale landforms is roughly NW to SE. S2 most closely equates with the $\mathrm{O} 2$ outwash landform defined by Pavey et al. (1999) and the Wisconsin low through high landforms of Morris and Pierce (1967). The suggested 18 to $15 \mathrm{ka}$ age for the O2 by Pavey et al., (1999) is supported in this study by an OSL age of 16,805+/-1175 (BG 4176) obtained from a 2Btx horizon within a silty unit underlying a thin $(0.4 \mathrm{~m})$ sand unit on a S2 ridge crest (Table 4-1; Fig. 4-4).

In addition to the 1937 high-water mark, a potential earlier S2 fluvial trim line is identified at 166 m (Fig. 4-3). Below this elevation, LiDAR data reflects a 'smoothed' appearance suggestive of fluvial planation, whereas above it relief remains complex. A distinctive S2 landform above $166 \mathrm{~m}$ is a $1.06 \mathrm{~km}^{2}$ low-relief closed oval basin. This basin is incised up to $9 \mathrm{~m}$ by Gilpin Run and has ca. $1 \mathrm{~m}$ of fine overbank sediments overlying moderately sorted, matrix-supported, low-angle cross-stratified gravels interpreted as fluvially reworked outwash. The comparative thinness of overbank sediments, coupled with low elevation and relief of the closed basin, suggests an erosional unconformity. An AMS radiocarbon age of $70+/-30 \mathrm{BP}$ (260 to 0 cal yrs BP; $\delta^{13} \mathrm{C}=-26.8$; Beta 442868) (Table 4-1; Fig. 4-4) from a 2Ab horizon of section CB4 likely dates the initiation of Euro-American land clearance activity and subsequent erosion sometime after 1800 A.D. 


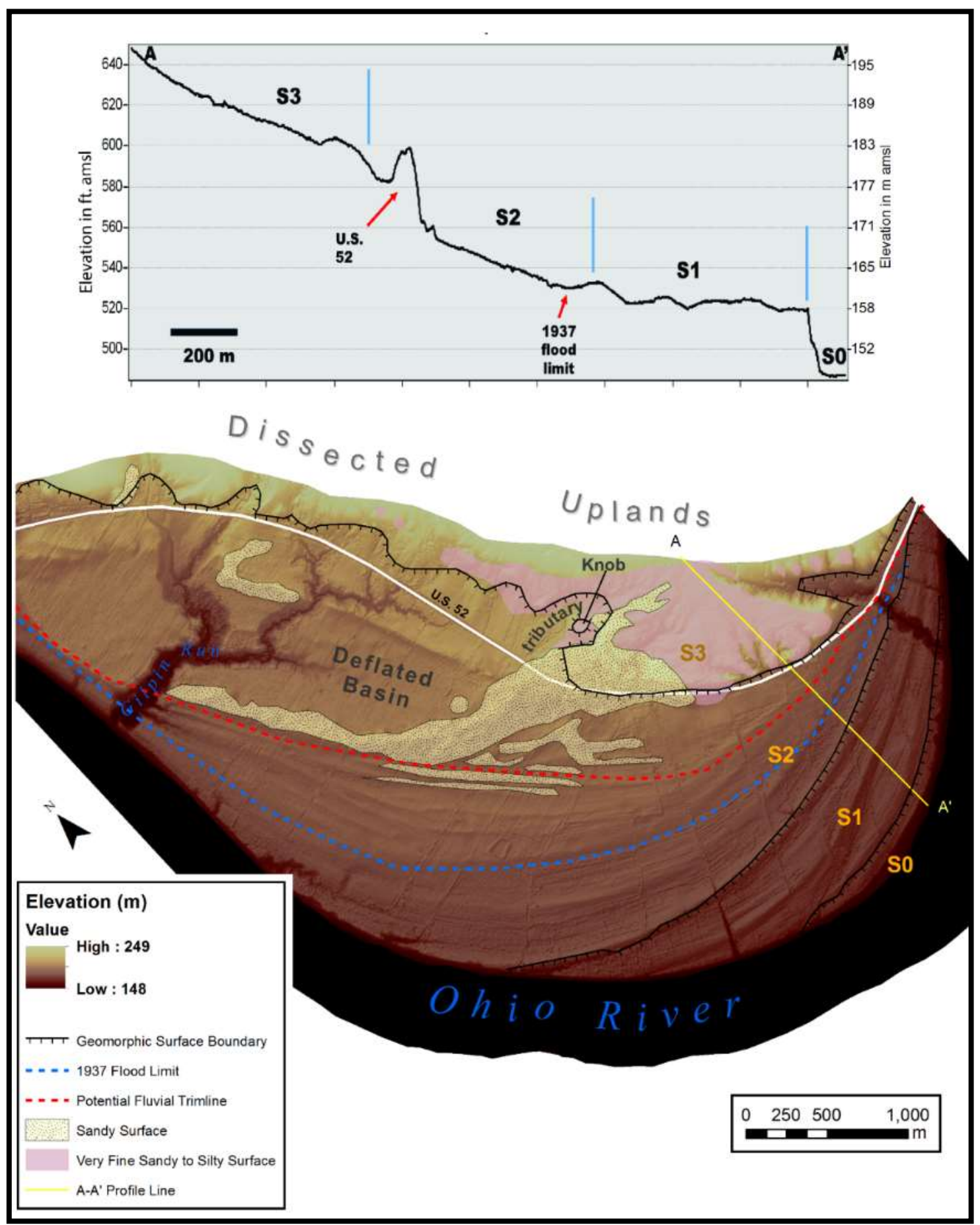

Fig. 4-3. Upper image: A-A' cross-section line showing defined geomorphic surfaces and general surface topography. Lower image: Sandy Springs LiDAR image showing distribution of various landforms, geomorphic surfaces (S0-S3), fluvial trim lines, and surficial distribution of sandy to very fine sandy to silty sediments. Depicted sediment texture distributions are based on geological mapping of Morris and Pierce (1967) as modified by the results of the current study. 
Intact outwash deposits were not encountered outside of the deflated basin boundary even in Giddings probes that reached ca. $4.5 \mathrm{~m}$ depth. Inspection of 20 water well logs in the immediate Sandy Springs area indicate sand and gravel deposits occur at various depths, but mostly $>2 \mathrm{~m}$ (ODNR, 2017). Isolated pockets of pebbles and cobbles are common on the S2 surface, both within swales and on ridges.

Above $166 \mathrm{~m}$, S2 contains an array of hummocky landforms dominated by sandy units of variable thickness. Morphology and landscape position allow classification of these landforms into several dune types including complex and compound dunes, a longitudinal dune, a compound barchan-like dune, a climbing dune, and an interdune sand sheet (see Fig. 4-2). Average dune heights range between 3 and $5 \mathrm{~m}$ west of U.S. 52, but increase up to $9 \mathrm{~m}$ in height east of U.S. 52 near the S2-S3 escarpment. The longitudinal dune east of U.S. 52 also contain high-angle $\left(>20^{\circ}\right)$ and low-angle planar cross-beds with ENE to N dip directions (Fig. 4-5, see also Supplemental Material). Planar cross-beds typically were identified at depths of ca.1 m or more, but locally were observed as shallow as $0.4 \mathrm{~m}$ (e.g., section CB1.1a). Cross-bed visibility was enhanced in beds with redox features where bedding was accentuated by secondary precipitates of Fe and Mn oxides.

Lamellae-accentuated cross-bedding was observed in the longitudinal and barchan dunes and have dips of $5^{\circ} \mathrm{NE}$ and $6^{\circ} \mathrm{ENE}$, respectively. These wavy lamellae accentuated beds, which are identified between 0.7 and $2.2 \mathrm{~m}$ below surface, morphologically resemble wind ripples that commonly occur on sand sheets or the stoss side of dunes and result in wind-ripple laminae (Bagnold, 1941; Hunter, 1977; G Kocurek, 1996). Micromorphological analysis of wavy lamellae, however, revealed well-oriented limpid clay domains that coat and bridge individual grains and fill pore space within lamellae (see Fig. 4-5C). In XPL, lamellae clay domains are birefringent and exhibit sharp extinction lines suggestive of illuvial translocation (Fedoroff, 1974; Stephen, 1960; Stoops, 2003: 19) and are interpreted as secondary pedogenic structures (J. Elmo Rawling, 2000). Lamellae formation and orientation likely were influenced by the position of primary bedding planes where subtle textural discontinuities facilitated deposition of clay translocated within wetting fronts (Schaetzl and Anderson, 2005: 368; Soil Survey Staff, 1999: 82). Particle-size analysis and micromorphology show $36 \%$ more clay in lamellae than interlamellae zones in the $2 \mathrm{Bt} \& \mathrm{E}$ horizon.

Sand units west of U.S. 52 reflect sand mantling of underlying alluvial ridge crests and are not true dune forms. Unlike sand sheets that are typically low relief, nearly featureless, and commonly winnowed of fine-textured sediments (Cooke et al., 1993), sand mantles are units of sand-textured sediments unconformably overlaying fine-textured alluvial sediments on a rolling ridge landform. The sand mantle units studied here were unstratified and ranged in thickness between 0.4 and $2 \mathrm{~m}$. It is likely that additional sandy, likely aeolian landforms once existed below the $164 \mathrm{~m}$ trim line but that past flood scouring and planation have eroded all traces of them. Four OSL ages indicate that S2 aeolian processes at Sandy Springs were active during the Holocene between 11.0 and 1.4 ka (Table 4-1; Fig. 4-4). Although Holocene ages only date the upper ca. $3 \mathrm{~m}$ of aeolian sediment for S2 landforms, the $17 \mathrm{ka}$ (BG 4176) OSL age of the underlying alluvial ridge crest at section CB6 constrains the initial timing of aeolian activity on this surface. 
Table 4-1. Results of OSL dating for Sandy Springs

\begin{tabular}{|c|c|c|c|c|c|c|c|c|c|c|c|c|}
\hline $\begin{array}{l}\text { Lab } \\
\text { number }\end{array}$ & $\begin{array}{l}\text { Section/ } \\
\text { Depth } \\
\text { (m) }\end{array}$ & $\begin{array}{l}\text { Facies } \\
\text { (Setting) }^{\mathrm{a}}\end{array}$ & Aliquots $^{\text {b }}$ & $\begin{array}{l}\text { Grain Size } \\
(\mu \mathrm{m})\end{array}$ & $\begin{array}{l}\text { Equivale- } \\
\text { nt dose } \\
\text { (Gray) }^{c}\end{array}$ & $\begin{array}{l}\text { Overdisp- } \\
\text { ersion } \\
(\%)^{\mathrm{d}}\end{array}$ & $\begin{array}{l}\mathrm{U} \\
()^{\mathrm{e} p m}\end{array}$ & $\begin{array}{l}\text { Th } \\
(p p m)^{e}\end{array}$ & $\begin{array}{l}\mathrm{K} \\
(\%)^{\mathrm{e}}\end{array}$ & $\begin{array}{l}\text { Cosmic dose } \\
\text { rate } \\
(\mathrm{mGray} / \mathrm{yr})\end{array}$ & $\begin{array}{l}\text { Dose rate } \\
(\mathrm{mGray} / \mathrm{yr})^{\mathrm{f}}\end{array}$ & OSL age $(y r)^{g}$ \\
\hline BG 4173 & $\begin{array}{l}\text { CB1/ } \\
1.25\end{array}$ & $\begin{array}{l}\text { aeolian unit I } \\
\text { (climbing dune) }\end{array}$ & $41 / 51$ & $425-355$ & $10.53 \pm 0.54$ & $23 \pm 3$ & $\begin{array}{l}0.77 \pm \\
0.01\end{array}$ & $\begin{array}{l}1.84 \pm \\
0.01\end{array}$ & $\begin{array}{l}0.58 \pm \\
0.01\end{array}$ & $0.185 \pm 0.019$ & $0.95 \pm 0.05$ & $11,055 \pm 820$ \\
\hline BG 4160 & $\begin{array}{l}\text { CB1a/ } \\
2.85\end{array}$ & $\begin{array}{l}\text { aeolian unit I } \\
\text { (climbing dune) }\end{array}$ & $60 / 71 / 8$ & $425-500$ & $4.00 \pm 0.23$ & $53 \pm 5$ & $\begin{array}{l}0.76 \pm \\
0.01\end{array}$ & $\begin{array}{l}1.72 \pm \\
0.01\end{array}$ & $\begin{array}{l}0.59 \pm \\
0.01\end{array}$ & $0.160 \pm 0.016$ & $0.90 \pm 0.05$ & $4450 \pm 350$ \\
\hline BG 4174 & $\begin{array}{l}\mathrm{U} 1 / \\
0.75\end{array}$ & $\begin{array}{l}\text { indeterminate (likely aeolian) } \\
(\mathrm{knob})\end{array}$ & $32 / 35 / 9$ & $355-250$ & $14.10 \pm 0.71$ & $37 \pm 5$ & $\begin{array}{l}2.00 \pm \\
0.01\end{array}$ & $\begin{array}{l}4.41 \pm \\
0.01\end{array}$ & $\begin{array}{l}0.92 \pm \\
0.01\end{array}$ & $0.192 \pm 0.019$ & $1.72 \pm 0.09$ & $8190 \pm 585$ \\
\hline BG 4161 & $\begin{array}{l}\mathrm{U} 2 / \\
0.76\end{array}$ & $\begin{array}{l}\text { mixed aeolian unit } 1 / \text { alluvial } \\
\text { unit } 1 \\
\text { (compound barchan dune) }\end{array}$ & $61 / 70 / 10$ & $425-355$ & $7.82 \pm 0.60$ & $48 \pm 4$ & $\begin{array}{l}2.04 \pm \\
0.01\end{array}$ & $\begin{array}{l}4.92 \pm \\
0.01\end{array}$ & $\begin{array}{l}088 \pm \\
0.01\end{array}$ & $0.196 \pm 0.020$ & $1.72 \pm 0.09$ & $4590 \pm 420$ \\
\hline BG 4175 & $\begin{array}{l}\mathrm{U} 3 / \\
0.85\end{array}$ & $\begin{array}{l}\text { untested (likely aeolian) } \\
\text { (sand sheet) }\end{array}$ & $28 / 35 / 6$ & $425-355$ & $7.43 \pm 0.40$ & $37 \pm 5$ & $\begin{array}{l}1.13 \pm \\
0.01\end{array}$ & $\begin{array}{l}2.70 \pm \\
0.01\end{array}$ & $\begin{array}{l}0.69 \pm \\
0.01\end{array}$ & $0.195 \pm 0.020$ & $1.19 \pm 0.06$ & $6235 \pm 470$ \\
\hline BG 4162 & $\begin{array}{l}\text { CB4/ } \\
1.71\end{array}$ & $\begin{array}{l}\text { alluvial unit II } \\
\text { (alluvial terrace tread) }\end{array}$ & $51 / 63 / 9$ & $425-355$ & $7.58 \pm 0.49$ & $44 \pm 4$ & $\begin{array}{l}1.83 \pm \\
0.01\end{array}$ & $\begin{array}{l}4.47 \pm \\
0.01\end{array}$ & $\begin{array}{l}1.05 \pm \\
0.01\end{array}$ & $0.177 \pm 0.018$ & $1.74 \pm 0.09$ & $4360 \pm 300$ \\
\hline BG 4176 & $\begin{array}{l}\text { CB6/ } \\
0.97\end{array}$ & $\begin{array}{l}\text { alluvial unit I } \\
\text { (alluvial ridge) }\end{array}$ & $23 / 26$ & 63-44 & $53.85 \pm 2.62$ & $21 \pm 3$ & $\begin{array}{l}3.93 \pm \\
0.01\end{array}$ & $\begin{array}{l}11.10 \pm \\
0.01\end{array}$ & $\begin{array}{l}1.49 \pm \\
0.01\end{array}$ & $0.192 \pm 0.019$ & $3.20 \pm 0.16$ & $16,805 \pm 1175$ \\
\hline BG 4163 & $\begin{array}{l}\text { BA8/ } \\
0.7\end{array}$ & $\begin{array}{l}\text { aeolian unit I } \\
\text { (alluvial ridge) }\end{array}$ & $56 / 63 / 6$ & $425-355$ & $2.56 \pm 0.13$ & $40 \pm 4$ & $\begin{array}{l}1.79 \pm \\
0.01\end{array}$ & $\begin{array}{l}2.88 \pm \\
0.01\end{array}$ & $\begin{array}{l}1.05 \pm \\
0.01\end{array}$ & $0.198 \pm 0.020$ & $1.61 \pm 0.08$ & $1400 \pm 110$ \\
\hline
\end{tabular}

a Facies units defined in Section 4.4. 'Untested' refers to a setting where particle-size analysis was not conducted. 'Indeterminate' refers to a sample that could not be classified into a distinct facies unit through PCA.

${ }^{\mathrm{b}}$ Aliquots used in equivalent dose calculations versus original aliquots measured.

${ }^{c}$ Equivalent dose calculated on a pure quartz fraction with about $40-100$ grains/aliquot and analyzed under blue-light excitation ( $\left.470 \pm 20 \mathrm{~nm}\right)$ by single aliquot regeneration protocols (Murray \& Wintle, 2003). The central age model of Galbraith et al., (1999) was used to calculated equivalent dose when overdispersion values are $<25 \%$ (at 1 sigma errors) A finite mixture age model was used with overdispersion values $>25 \%$ to determine the youngest equivalent dose population, which is the third value listed. ${ }^{\mathrm{d}}$ Values reflects precision beyond instrumental errors; values of $\leq 25 \%$ (at 1 sigma limit) indicate low dispersion in equivalent dose values and an unimodal distribution.

eU, Th and $\mathrm{K}$ content analyzed by inductively-coupled plasma-mass spectrometry analyzed by ALS Laboratories, Reno, NV; U content includes Rb equivalent.

${ }^{\mathrm{f}}$ Cosmic dose rate calculated from parameters in Prescott and Hutton (1994).

${ }^{\mathrm{g} S y s t e m a t i c}$ and random errors calculated in a quadrature at 1 standard deviation. Datum year is AD 2010. 


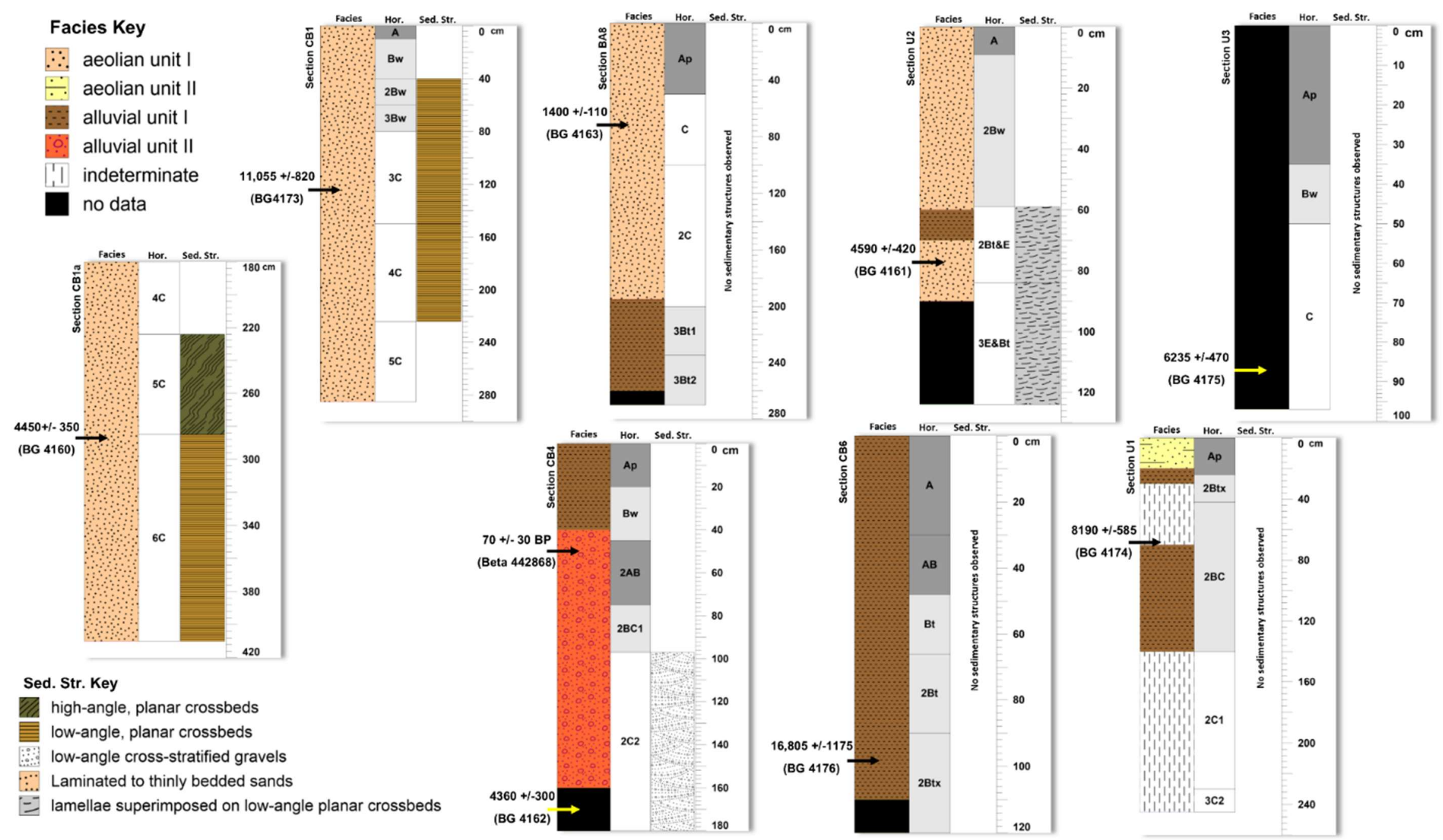

Fig. 4-4. Stratigraphic sections showing facies, soil horizons, sedimentary structures, and OSL or AMS radiocarbon ages for key locations (see Fig. S6 and S7 for detail on section locations). 
Two OSL ages that exhibit an age reversal with depth in the section CB1/1a profile of the S2 longitudinal dune deserve discussion (see Fig. 4-4). An OSL age of ca. 11 ka was obtained from $1.25 \mathrm{~m}$ and was characterized by low-angle planar cross beds. Approximately $3 \mathrm{~m}$ to the north, a second lower section of the same dune yielded an OSL age of ca. $4.5 \mathrm{ka}$ at a depth of $2.85 \mathrm{~m}$. This significantly younger age was associated with high-angle $\left(33^{\circ}\right)$ planar cross-beds with NNE dip.

Since no statistical justification is present to reject either OSL result, we interpret the age reversal between these two portions of the section as evidence of erosional undercutting and reactivation of the previously stabilized longitudinal dune. In this scenario, significant dune construction occurred prior to ca. $11 \mathrm{ka}$ and sedimentation likely ceased soon after. The lowangle, cross-bedding observed at $1.25 \mathrm{~m}$ likely reflects topset stratification. By $4.5 \mathrm{ka}$, the northern toe of the longitudinal dune eroded perhaps in response to gully incision associated with a natural spring approximately $15 \mathrm{~m}$ to the north (Purtill, 2017: 166). If this scenario is correct, erosional activity undercut and destabilized the NNE side of the dune causing reactivation of a slip face through grain flow. Additional evidence for this reactivation comes from the $4.5 \mathrm{ka}$ OSL sample where an overdispersion equivalent dose value of 53+/-5\% strongly suggests shortterm sediment transport (Forman, 2015: 5).

\subsection{S3 Geomorphic Surface}

The S3 surface rises from 39 to $46 \mathrm{~m}$ above the Ohio low-water elevation. This surface abuts dissected bedrock uplands to the east and has an eroded, discontinuous boundary to the west. The S3 surface is incised deeply by several unnamed ephemeral drainages and gradually slopes toward the Ohio River at a 2.8\% slope. Portions of the S3 riser are weathered and rounded; whereas, a prominent 3-m escarpment defines the southern boundary. An isolated erosional S3 remnant herein referred to as the knob (Fig. 4-3) is adjacent to a climbing sand dune landform. The S3 surface corresponds to Pavey et al.'s (1999) O1 outwash landform and Morris and Pierce's (1967) high Illinoian landform. Based on particle-size data and an OSL age of $8.2 \mathrm{ka}$ (BG 4174), study results support Morris and Pierce's (1967) interpretation of a ca. $1 \mathrm{~m}$ mantle of primarily unstratified aeolian sediment on this high surface and demonstrates that aeolian deposition occurred into the early Holocene. S3 sediments below ca. $1 \mathrm{~m}$ on the knob remain undated and show a mixture of alluvial and aeolian deposition with local laminated sands observed in some sections.

\subsection{Particle-size analysis and facies definition}

Of the 203 sediment samples retrieved during fieldwork, 194 were subject to particle-size and statistical analysis $\left(M d_{\varphi}, \bar{x}_{\varphi}, \sigma_{\varphi}, S k, K\right.$, and $\left.C V\right)$. Table 4-3 summarizes statistical results of the complete $\varphi$ range for 84 samples from the upper-most lithological units by landform type. This selective approach was adopted since the upper-most lithological unit best represents the processes that shaped current landforms. Complete statistical calculations by sample location are available in Supplementary Material, Appendix C. 

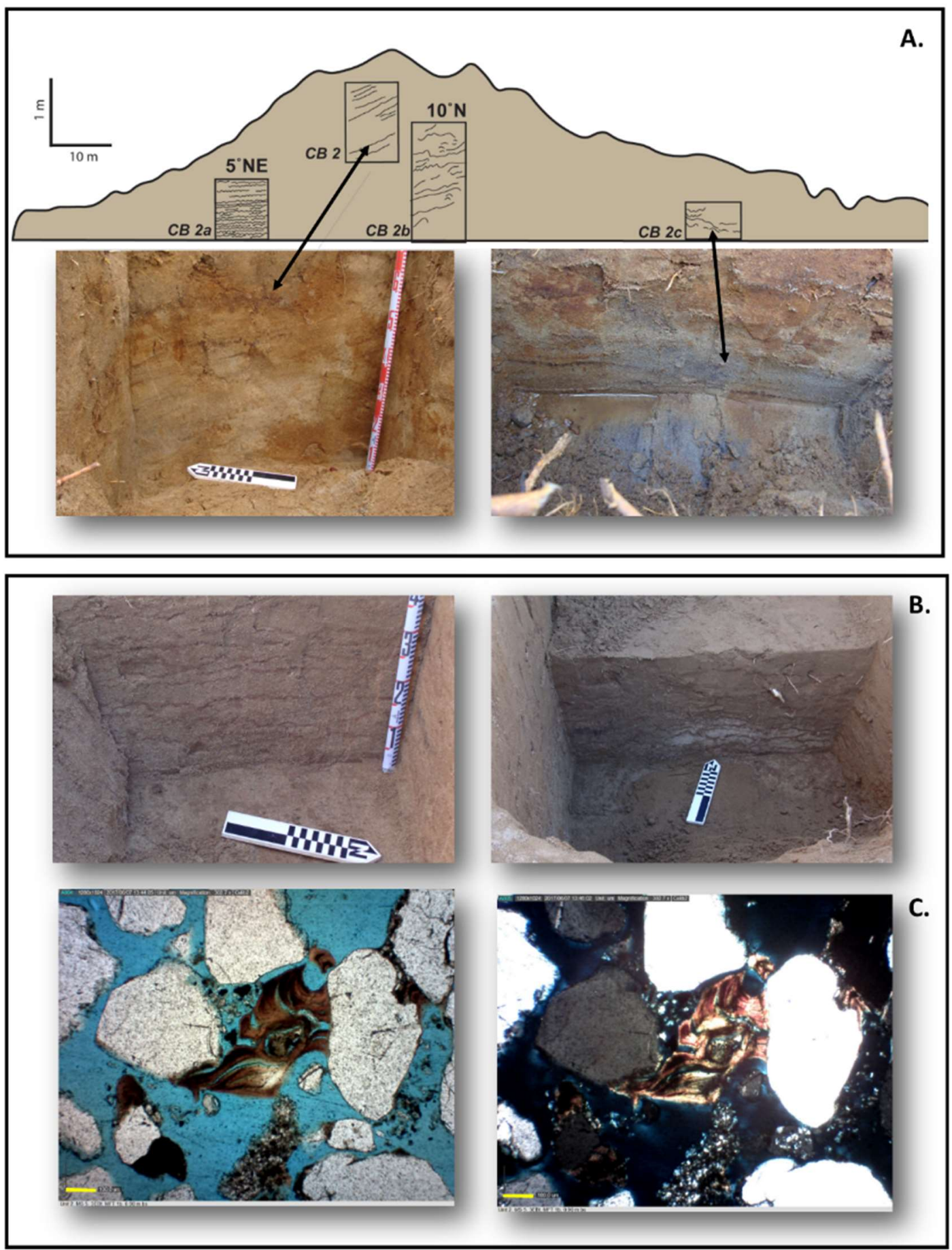

Fig. 4-5. Examples of primary planar cross-beds and secondary lamellae structures in dunes. A. East profile of longitudinal dune at U.S. 52 road cut showing bedding planes true dip angle and direction. B. View of secondary lamellae within compound barchan-like dune. Scale north arrow illustrates direction of magnetic north. C. PPL (left) and XPL (right) micrographs of welloriented illuviated clay domains linking quartz grains within lamellae band of 3E\&Bt horizon at $0.9 \mathrm{~m}$ depth in compound barchan-like dune. Note sharp extinction lines for clay domains in XPL. Yellow bar on micrographs represents 100 microns. 
A multivariate PCA was calculated to determine if depositional environments could be ascertained from particle-size data at Sandy Springs. The PCA was calculated for all 18 particlesize statistics outlined in Section 3.3 to identify parameters most responsible for assemblage variance. The appropriateness of PCA analysis with this dataset was confirmed initially through the Kaiser-Meyer-Olkin measure (0.752) and Bartlett's Test of Sphericity $\left(\chi^{2}=6868.89 ; d f=\right.$ 153; $p<0.001$ ) (Field, 2009: 647-648). Three factors with eigenvalues $>1$ were retained and account for 81 percent of assemblage variability. Variables with loadings $>0.4$ (Field, 2009: 666; Stevens, 2002) are shown in Table 4-2. The following statistics have high factor scores and

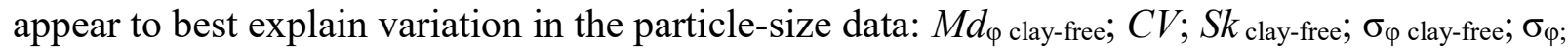
$K$; and $C V_{\text {sand }}$ (Table 4-3).

Table 4-2. Particle-size characteristics (complete $\varphi$ range) by landform type for uppermost samples in each lithological unit

\begin{tabular}{|l|r|r|r|r|r|r|r|}
\hline \multicolumn{1}{|c|}{ Landform } & $\begin{array}{c}\text { Sample } \\
\text { Size }\end{array}$ & $\boldsymbol{M d}_{\boldsymbol{\varphi}}$ & $\overline{\boldsymbol{x}}_{\boldsymbol{\varphi}}$ & $\boldsymbol{\sigma}_{\boldsymbol{\varphi}}$ & $\boldsymbol{S k}$ & $\boldsymbol{K}$ & $\boldsymbol{C V}(\boldsymbol{\%})$ \\
\hline Compound Barchan Dune & 3 & 1.64 & 2.44 & 2.40 & 2.54 & 9.10 & 97.85 \\
\hline Sand Dune, Longitudinal Dune & 9 & 1.46 & 1.82 & 1.76 & 3.10 & 15.67 & 94.14 \\
\hline Sand Dune, Climbing Dune & 4 & 1.53 & 1.96 & 1.82 & 3.62 & 18.30 & 90.62 \\
\hline Sand Dune/Gully, Climbing Dune & 3 & 1.89 & 3.26 & 2.61 & 1.13 & 3.31 & 80.03 \\
\hline Sand Sheet & 15 & 1.46 & 1.85 & 1.72 & 3.11 & 14.59 & 90.78 \\
\hline Knob & 6 & 4.79 & 5.22 & 2.52 & 0.70 & 2.79 & 49.82 \\
\hline Alluvial Ridge (sand mantle) & 14 & 3.59 & 4.24 & 2.74 & 1.15 & 4.44 & 71.74 \\
\hline Alluvial Swale & 1 & 1.52 & 2.10 & 2.15 & 2.73 & 10.00 & 102.34 \\
\hline Alluvial Terrace Tread & 18 & 5.61 & 6.13 & 2.86 & 0.12 & 2.06 & 47.91 \\
\hline Tributary Drainage & 11 & 5.62 & 6.07 & 2.65 & 0.17 & 2.51 & 44.43 \\
\hline
\end{tabular}

Factor 1 and 2 scores plotted by geomorphic setting demonstrate strong spatial clustering with minimal overlap along two distinct axes (Fig. 4-6). At least three patterns are evident. First, sand dune and interdunal sand sheet sediments show little variation and plot almost exclusively along a single axis. Second, alluvial sediments plot along on second axis but in a slightly more dispersed pattern than dune sediments. Third, the upper 2 to $3 \mathrm{~m}$ of sediment on the knob landform tend to disperse between sand dune and alluvial axes and fail to show a strong pattern.

Initial plotting of PCA factors, along with consideration of geomorphic context, grain characteristics, particle size cumulative graphs, and chronometric ages, were used to define six sedimentary facies: aeolian units I and II; colluvial unit I; alluvial units I, II, III, and IV (Table 44). A seventh indeterminate unit was created for samples failing to fit the six defined facies. Although sample plotting shows strong agreement between geomorphic setting and hypothesized depositional environment (e.g., most sand dune sediments plot on the aeolian unit I axes), sample plot position was used to reclassify some samples. For example, some alluvial ridge samples, initially assumed to reflect alluvial unit I, actually plot on the aeolian unit I axes in Fig. 4-6. Based on plot position, these samples are reinterpreted for this study as windblown sediments and reclassified as aeolian unit I. Similarly, compound barchan-like dune samples plot primarily on the aeolian unit I axes but several plot along the alluvial unit I axes suggesting some aeolian- 
fluvial inter-mixing is responsible for current landform morphologies. Following assignment of facies type, samples were re-plotted by factor scores in Fig. 4-7. Table 4-4 provides summary statistics for the complete $\varphi$ range by facies type for all samples.

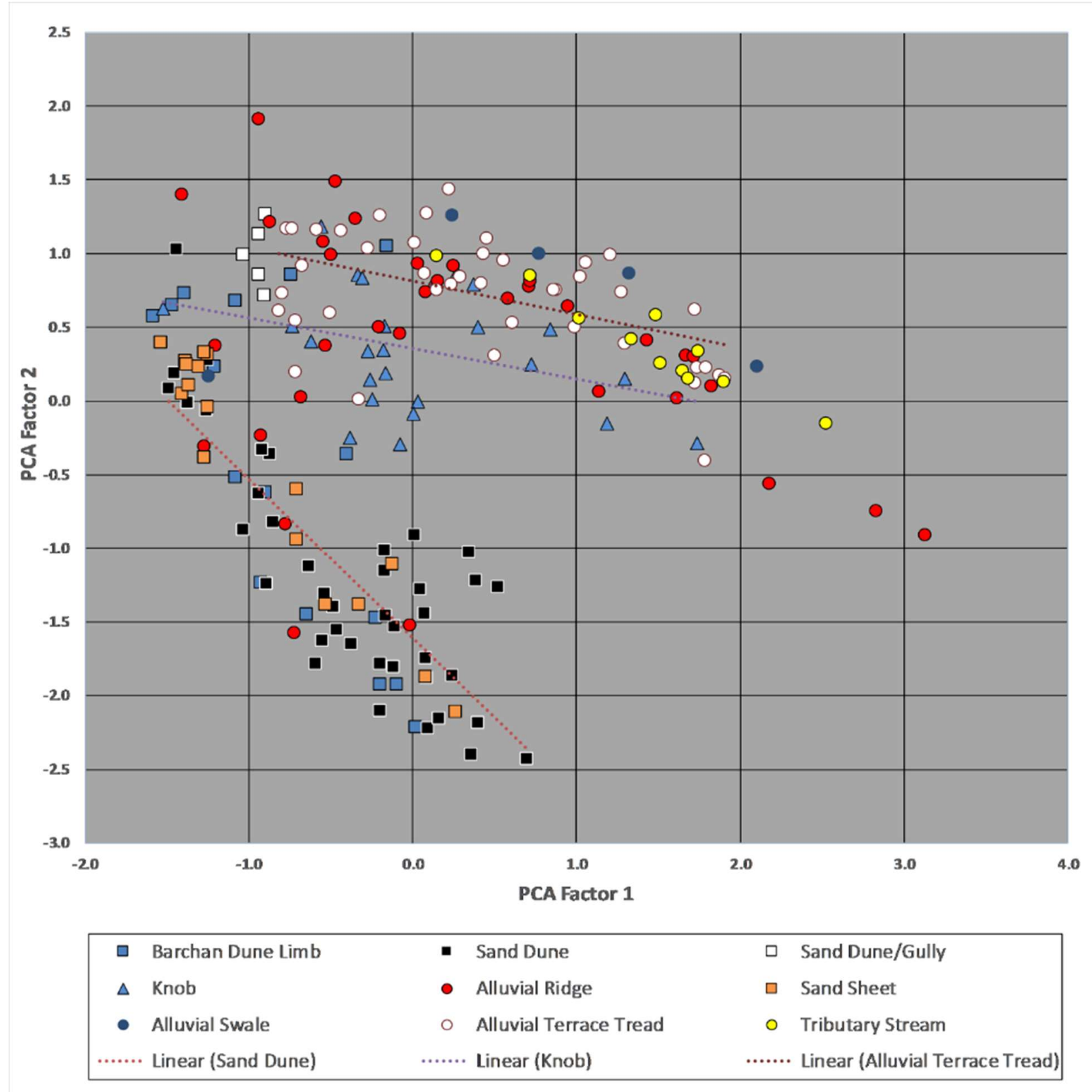

Fig. 4-6. Initial bi-plot of PCA Factors 1 and 2 by geomorphic context showing strong clustering of sand dune and alluvial samples along distinct axes. Trend lines shown for sand dune, knob, and alluvial terrace tread samples. Variables used to create each factor are detailed in Table 4-3. 
Table 4-3. PCA rotated factor scores (varimax rotation with Kaiser normalization)

\begin{tabular}{|l|r|r|r|}
\hline Variables & $\begin{array}{c}\text { PCA } \\
\text { Factor 1 }\end{array}$ & $\begin{array}{c}\text { PCA } \\
\text { Factor 2 }\end{array}$ & $\begin{array}{c}\text { PCA } \\
\text { Factor 3 }\end{array}$ \\
\hline$M d_{\varphi \text { clay-free }}$ & .851 & .422 & -.162 \\
\hline$C V$ & -.831 & -.038 & .284 \\
\hline$S k_{\text {clay-free }}$ & -.815 & -.347 & -.003 \\
\hline$M d_{\varphi}$ & .801 & .501 & -.101 \\
\hline $\bar{x}_{\varphi \text { clay-free }}$ & .799 & .530 & -.170 \\
\hline$\sigma_{\varphi \text { sand }}$ & .779 & .511 & .160 \\
\hline$C V_{\text {clay-free }}$ & -.755 & .315 & .467 \\
\hline $\bar{x}_{\varphi}$ & .740 & .606 & -.145 \\
\hline$K_{\text {sand }}$ & -.575 & -.463 & .108 \\
\hline$\sigma_{\varphi \text { clay-free }}$ & .148 & .902 & .183 \\
\hline$\sigma_{\varphi}$ & .122 & .901 & .046 \\
\hline$K$ & -.208 & -.859 & .059 \\
\hline$S k$ & -.489 & -.787 & .024 \\
\hline$K_{\text {clay-free }}$ & -.377 & -.651 & .030 \\
\hline$C V_{\text {sand }}$ & .335 & .218 & .838 \\
\hline$M d_{\varphi \text { sand }}$ & .445 & .381 & -.770 \\
\hline $\bar{x}_{\varphi \text { sand }}$ & .479 & .441 & -.717 \\
\hline$S k_{\text {sand }}$ & -.304 & .228 & .503 \\
\hline
\end{tabular}

Sediment from aeolian facies (aeolian units I and II) collectively have a mean size of $2.2 \varphi$, are moderately sorted $\left(\sigma_{\varphi}=1.7 \varphi\right)$, have a very coarse positive skew $(S k=3.2)$, are very leptokurtic $(K=17.1)$, and have an average $C V$ of $80.1 \%$. Micromorphological analysis of aeolian facies at Sandy Springs revealed sub-rounded, low sphericity grain morphologies. Only $7 \%$ of grains interpreted as aeolian are well-rounded with high sphericity, a morphology traditionally associated with far-traveled aeolian dune sands (Pye and Tsoar, 2009: 82). In general, textural parameters from Sandy Springs compare closely with other studies of aeolian sediments (e.g., Friedman, 1979, 1961; Ivester and Leigh, 2003; Leigh, 1998) with some notable exceptions. Aeolian sediments at Sandy Springs tend to be slightly finer, less well sorted, and have stronger positive skew and kurtosis than previously reported (e.g., Friedman, 1961; Ivester and Leigh, 2003: 302-303; Leigh, 1998: 318-321). For the $\varphi$ clay-free fraction along the Coastal Plain, Leigh (1998: 319-320) reported that $C V$ values between $40 \%$ and $70 \%$ clearly distinguished aeolian from other sediments which ranged between $70 \%$ and $140 \%$. Although $C V$ also distinguishes aeolian from non-aeolian sediments at Sandy Springs, both for complete $\varphi$ and $\varphi$ clay-free fractions (see Supplementary Material, Appendix C), the data trend is reversed in this study such that aeolian deposits display greater dispersion about the mean than non-aeolian sediments (see Table 4-4). This reversal may reflect variation in environmental settings between the glaciofluvial valley at Sandy Springs and unglaciated Coastal Plain sites (Leigh, 1998). 
Table 4-4. Particle-size description and characteristics (complete $\varphi$ range) by facies type

\begin{tabular}{|l|r|l|r|r|r|r|r|r|}
\hline Facies & \multicolumn{1}{|l}{ Size } & $\begin{array}{l}\text { General Description and } \\
\text { Interpretation }\end{array}$ & $\boldsymbol{M d}_{\boldsymbol{\varphi}}$ & $\overline{\boldsymbol{x}}_{\boldsymbol{\varphi}}$ & $\boldsymbol{\sigma}_{\boldsymbol{\varphi}}$ & $\boldsymbol{S k}$ & $\boldsymbol{K}$ & $\boldsymbol{C V}$ \\
\hline aeolian unit I & 82 & $\begin{array}{l}\text { Dunes and sand-mantled ridges, } \\
\text { high and low angle planar cross } \\
\text { beds and lamellae in dunes }\end{array}$ & 1.5 & 1.8 & 1.6 & 3.4 & 18.6 & 83.7 \\
\hline aeolian unit II & 9 & $\begin{array}{l}\text { Fine-textured coversands, } \\
\text { unstratified }\end{array}$ & 4.8 & 5.2 & 2.4 & 0.8 & 3.3 & 46.9 \\
\hline colluvial unit I & 5 & $\begin{array}{l}\text { Aeolian and alluvial sediments } \\
\text { reworked by gravity, } \\
\text { unstratified }\end{array}$ & 1.8 & 3.2 & 2.6 & 1.2 & 3.5 & 82.5 \\
\hline alluvial unit I & 69 & $\begin{array}{l}\text { Ohio River fine-grained } \\
\text { alluvium, rare laminated sands }\end{array}$ & 4.6 & 5.2 & 2.8 & 0.6 & 2.7 & 56.8 \\
\hline alluvial unit II & 3 & $\begin{array}{l}\text { Ohio River coarse-grained } \\
\text { alluvium, gravel cross beds }\end{array}$ & 3.5 & 3.8 & 3.4 & 0.7 & 2.9 & 103.0 \\
\hline alluvial unit III & 3 & $\begin{array}{l}\text { Ohio River fine-grained swale } \\
\text { alluvium, unstratified }\end{array}$ & 7.4 & 7.2 & 2.9 & -0.4 & 2.3 & 41.3 \\
\hline alluvial unit IV & 11 & $\begin{array}{l}\text { Tributary fine-grained } \\
\text { alluvium, unstratified }\end{array}$ & 5.6 & 6.1 & 2.6 & 0.2 & 2.5 & 44.4 \\
\hline indeterminate & 12 & - & 2.4 & 3.1 & 2.2 & 2.2 & 7.8 & 71.4 \\
\hline Total & $\mathbf{1 9 4}$ & - & $\mathbf{3 . 2}$ & $\mathbf{3 . 7}$ & $\mathbf{2 . 2}$ & $\mathbf{1 . 9}$ & $\mathbf{9 . 8}$ & $\mathbf{6 9 . 1}$ \\
\hline
\end{tabular}

Sediments assigned to aeolian unit I facies form dunes, sand sheets, and sand-mantled ridge landforms. The medium sand texture suggests short-distance suspension, surface traction and saltation as primary sediment transport modes (Pye and Tsoar, 2009: 113-115). The upper meter of the low-relief sand sheet has been accumulating since $6.2 \mathrm{ka}$ (see Table 4-1) likely through ripple bed accretion (G Kocurek, 1996; Pye \& Tsoar, 2009) although such sedimentary structures were not observed. In the eastern part of Sandy Springs, aeolian sediments are deposited in several dunes that abut the $\mathrm{S} 3$ scarp. Aeolian landforms rise up to $9 \mathrm{~m}$ above current alluvial surfaces and may be as much as $18 \mathrm{~m}$ in total thickness (Morris \& Pierce, 1967). West of U.S. 52 on the $\mathrm{S} 2$ terrace tread, aeolian unit I thinly mantles pre-existing alluvial ridge crests and are not true dunes. Sand mantles are unstratified and range in thickness between 0.4 and $2.0 \mathrm{~m}$ and PCA results suggest mixed aeolian-fluvial depositional processes especially west of U.S. 52. Aeolian unit I sediments are brown to yellowish brown with hues ranging from 8.1 YR to 0.8 Y. Most samples are sand although muddy sand, silty sand, and clayey sand sediments also occur. OSL ages from aeolian unit I sediments at Sandy Springs suggest deposition primarily during the Holocene (see Table 4-1 and discussion above). It is noteworthy that OSL ages only date the upper $3 \mathrm{~m}$ of landforms and likely reflect dune remobilization not initial deposition. 


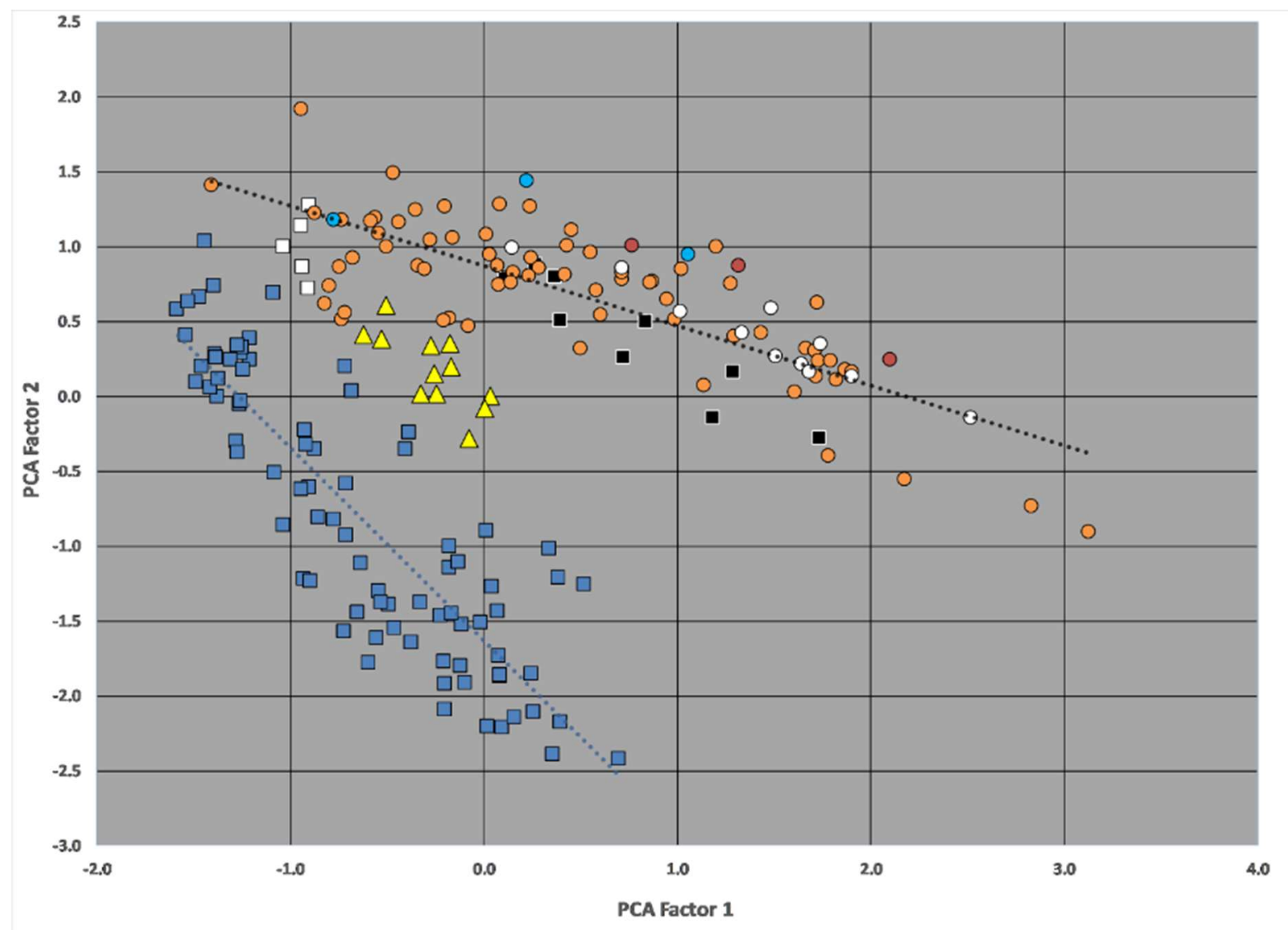

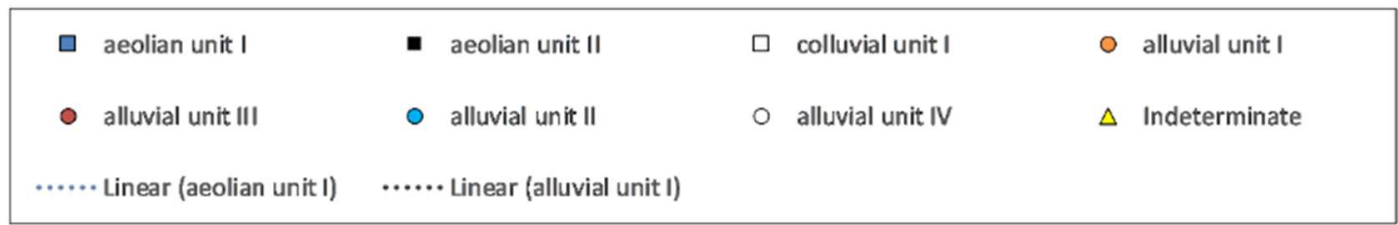

Fig. 4-7. Reclassification of sediment samples by facies type. Trend lines shown for aeolian unit I and alluvial unit I facies.

The aeolian unit II facies is unstratified sandy silt that blanket portions of older deposits on the S3 surface (see also Morris and Pierce, 1967). The fact that aeolian unit II mantles older landforms and has sand content that averages greater than $25 \%$ suggests this unit represents a fine-textured coversand deposit (Pye \& Tsoar, 2009; Schwan, 1986; Singhvi, Bluszcz, Bateman, $\&$ Rao, 2001). The very fine sand to silt texture at Sandy Springs suggests modified saltation and short-distance suspension as primary transport modes (Pye and Tsoar, 2009: 113-115). Sediments commonly are dark reddish brown with hues between 8.3 YR and 9.7 YR. On the knob, aeolian unit II facies also occur at depths between 1.6 and $3.9 \mathrm{~m}$ in section GP5, suggesting earlier undated aeolian deposition. Colluvial unit I was identified along the southern knob edge within an extant spring-fed gully (Purtill, 2017: 166). It is defined from five samples and appears to represent a colluvial mixture of aeolian unit I and alluvial unit I sediments and share characteristics of each facies (see Table 4-4). 
Alluvial sediment (alluvial units I - IV) has a mean size of $5.4 \varphi$ and is poorly sorted $\left(\sigma_{\varphi}=2.8 \varphi\right.$ ). Alluvial sediment has a coarse positive skew $(S k=0.5)$, is mesokurtic $(K=2.7)$, and has an average $C V$ of $56.3 \%$. Alluvial unit I sediment occurs broadly across $\mathrm{S} 2$ on alluvial ridge and tread landforms. In some S3 surface profiles, this facies underlies aeolian unit II, often as unweathered, laminated to thinly bedded sands. Sediments tend to be sandy mud to silty sand and range in color from reddish (7.6 YR) to yellowish brown (9.9 YR) hues.

Alluvial units I and IV sediments were defined from a limited number of S2 samples. In both cases, facies properties are similar to typical alluvial unit I sediments except for the tendency to be more fine-textured, symmetrical, and platykurtic (see Table 4-4). Texture ranges from a sandy silt to a sandy mud for tributary sediments and sandy mud to mud for swale sediments. Colors for both facies were brown to yellowish brown with hues between $9.5 \mathrm{YR}$ and $0.5 \mathrm{Y}$. A fluctuating water table in the tributary basin sediments is suggested by common redoximorphic features.

Although the PCA analysis was broadly successful at distinguishing aeolian from alluvial sediments based on grain characteristics, considerable overlap in plotted factor scores exists among some facies. For example, sediments identified as aeolian unit II facies and alluvial unit I facies overlap considerably (Fig. 4-7). These facies, however, generally are distinguishable in $\varphi$ cumulative distribution graphs (Fig. 4-8). Aeolian unit I, illustrated for comparison in figure 8, is characterized by a consistent pattern with $>70$ percent of sediment in the 1-2 $\varphi$ size range. In contrast, alluvial unit I sediments show considerable variability with a less consistent pattern.

\subsection{Discussion}

\subsection{Alluvial Landform Development}

In the upper Ohio Valley, the Ohio River flows through a semi-confined valley where channel meandering is bedrock constrained. In reaches where the valley broadens, such as at Sandy Springs, the river meanders more freely and alluvial surfaces have developed through lateral accretion and overbank sedimentation. Significant lateral migration is evident at Sandy Springs for the Holocene S1 and Pleistocene S2 surfaces, where a gradual slope with ridge-and-swale topography resemble slip-off terraces (Leopold et al., 1964: 461). Fine overbank sediment, ranging in thickness between $1 \mathrm{~m}$ in the deflated basin and $>4.5 \mathrm{~m}$ elsewhere, characterize the upper sediment units of S2. Overbank sediments on S2, and presumably on other surfaces, are underlain by sandy gravel interpreted as fluvially reworked outwash (Morris \& Pierce, 1967; Pavey et al., 1999). 

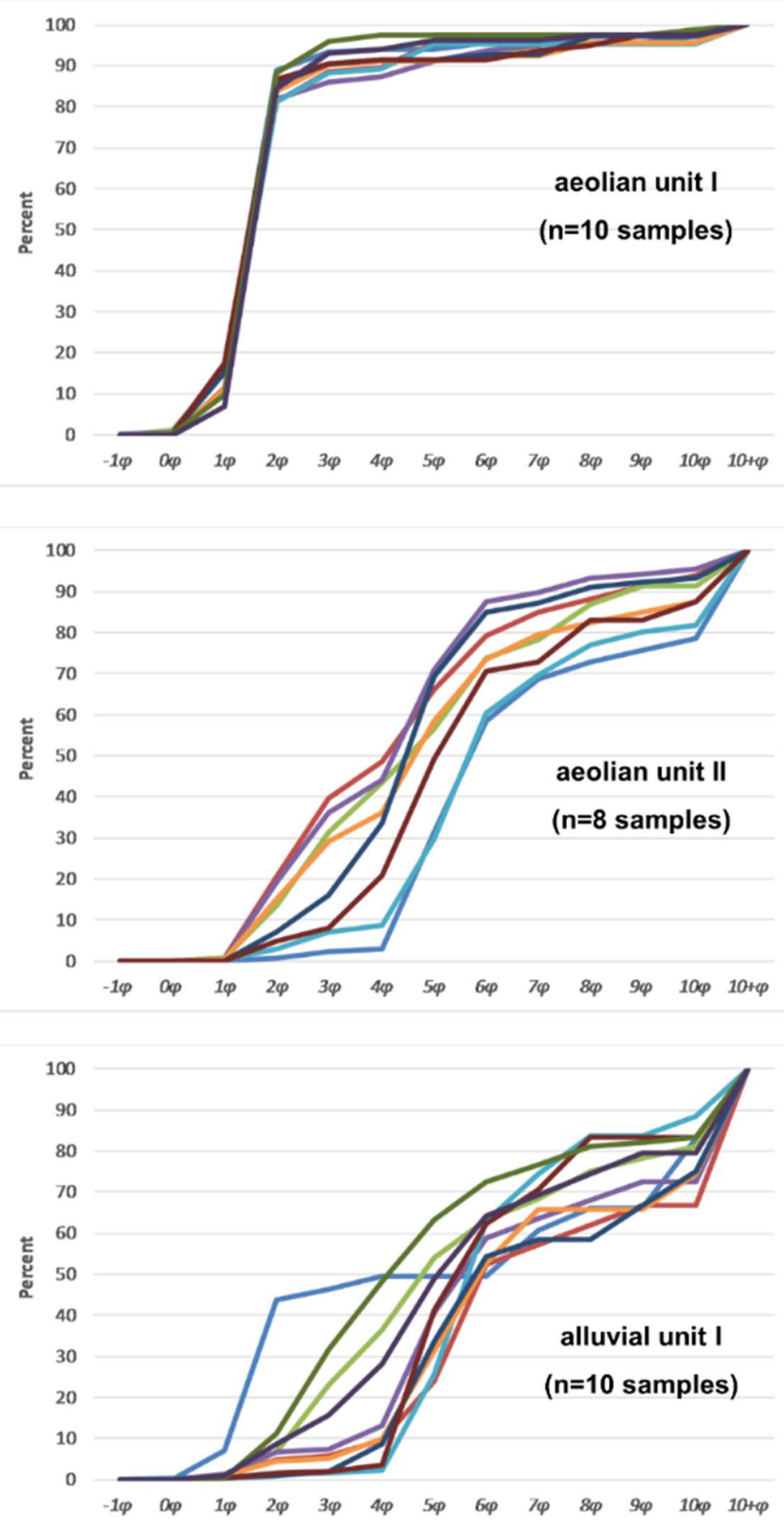

Fig. 4-8. Cumulative graphs $(\varphi)$ of particle-size data comparing aeolian units I and II, and alluvial unit I.

The rotation of alluvial ridge and swale orientation between S1 and S2 (Figs. 4-2 and 4-3) shows that an upstream translation of the Ohio River channel has been occurring since the late Pleistocene. The fact that translation is occurring upstream, instead of a more typical downstream migration pattern (Charlton, 2008: 142-143; Fryirs and Brierley, 2013: 184-185), 
may be related to downriver bedrock-influenced valley confinement or to unrecognized factors. This translation pattern is resulting in downstream erosion of the S2 surface near Gilpin Run. Historically, large floods have inundated significant portions of the S2 surface, perhaps as high as $166 \mathrm{~m}$ where a fluvial trim line is recognized in LiDAR data.

The highest and oldest alluvial surface recognized at Sandy Springs, S3, reflects a heavily eroded, discontinuous landform abutting dissected bedrock uplands. Tributary erosion has isolated a relict portion of the S3 as a knob that mistakenly was mapped as Plainfield series dune sand (Lucht \& Brown, 1994). The knob and other segments of the S3 surface (Morris \& Pierce, 1967) are mantled with fine sandy material interpreted as coversands deposited well into the Holocene at $8.2 \mathrm{ka}$ and perhaps later. A precise depositional age and history of underlying S3 facies remain undated and poorly understood. Particle-size data from knob sediments suggests a complex depositional history including both aeolian and fluvial construction of the upper $4.5 \mathrm{~m}$ of the S3 surface.

\subsection{Aeolian Landform Development}

Consistent with earlier upper Ohio Valley studies (e.g., Chappell, 1988; Rutledge et al., 1975; Simard, 1989), aeolian deposits and landforms are restricted to the older S2 and S3 surfaces at Sandy Springs. Aeolian sedimentation, interpreted here as some level of dune reactivation, was active during the Holocene between 11 and $1.4 \mathrm{ka}$. The $\sim 17 \mathrm{ka}$ age on alluvial ridge-and-swale topography on $\mathrm{S} 2$ constrains the timing of $\mathrm{S} 2$ aeolian activity.

Aeolian-fluvial compound dunes and sand-mantled ridges that partially rim the S2 deflated basin at Sandy Springs morphologically resemble Carolina bay systems in coastal environments (e.g., Markewich and Markewich, 1994; Pye, 1982), or pan-lunette systems in arid environments (e.g., Shaw and Bryant, 2011). Hypotheses for the formation of these systems vary, but most imply upwind aeolian deflation of topographic lows during periods of reduced soil moisture or watertable drawdown and immediate downwind deposition (cf., Goudie and Thomas, 1986). In semihumid or humid climates, sediment transport is influenced by vegetated terrain that encourages deposition and continued accretion of aeolian sediments. These landforms often are termed coppice or source-bordering dunes and may exhibit complex surface morphologies (Cohen et al., 2010; B. Liu \& Coulthard, 2015; Markewich \& Markewich, 1994; Moore \& Daniel Jr., 2011).

We suggest similar processes formed aeolian landforms at Sandy Springs. First, at the ca. 23 ka maximum extent of the Scioto and Miami sublobes of the Laurentide Ice Sheet (Fullerton, 1986; Glover et al., 2011) and during any subsequent advances-retreats, the upper Ohio River would have transported significant volumes of glacial outwash, especially immediately downriver of major outlet tributaries such as the Scioto River (Kempton \& Goldthwait, 1959; L. L. Ray, 1974). Although the transition of Ohio River from a braided to single-thread system is commonly placed between $20 \mathrm{ka}$ and $12 \mathrm{ka}$ (Glover et al., 2011; Mandel, 1988; Purtill, 2012: 2141; Ray, 1974), stratigraphic and radiocarbon evidence from $160 \mathrm{~km}$ upriver near Apple Grove, West Virginia, potentially suggests a sediment-rich braided pattern as late as $8.5 \mathrm{ka}$ (Rogers, 1990). The relatively wide, sinuous, and low gradient Ohio River valley between Garrison, Kentucky, and Sandy Springs would have reduced river flow strength and transport capacity (Fryirs and Brierley 2013: 70-71; Leopold et al. 1964: 271-317) resulting in a glaciofluvial 
sediment sink for fine sediments especially at significant river bends such as Sandy Springs. Finally, the glaciofluvial sediments deposited at Sandy Springs would have been conducive to aeolian transport due to the long local wind fetch which would have increased the potential for erosion (G Kocurek, 1996; Gary Kocurek \& Lancaster, 1999). Grain shape and sorting along with high OSL overdispersion values $(>30 \%)$ for Sandy Springs sediments are consistent with the view that aeolian sediments are locally sourced (Forman, 2015: 5; Pye and Tsoar, 2009: 82). Deflation of underlying fluvially reworked outwash on the deflated basin on S2, likely in response to some combination of reduced vegetation cover and groundwater drawdown associated with incision of Gilpin Run, is the likely source of sands and silts for S2 dunes and sand mantles as well as S3 coversands.

S2 aeolian deposits are thickest east of U.S. 52, where a longitudinal dune transitions to a $9 \mathrm{~m}$ high climbing dune that abuts the $\mathrm{S} 3$ escarpment. The orientation and thickness of these crossbedded dunes appear windswept and aligned with modern winter WSW winds. The fact that S2 longitudinal and climbing dunes, and the interdunal sand sheet, exhibit coarse textures $\left(\bar{x}_{\varphi}<2.0\right.$ $\varphi)$ suggests winnowing of fines and deposition immediately downwind as fine-textured coversands $\left(\bar{x}_{\varphi}=5.5 \varphi\right)$ on the elevated $\mathrm{S} 3$.

Unlike thick aeolian units east of U.S. 52, modern prevailing wind directions fail to fully explain sand mantling on alluvial ridge crests and formation of compound dunes on the western upwind edge of the S2 deflation basin. Based on particle-size data, we interpret these landforms as resulting from a complex interplay of aeolian-fluvial depositional and erosional processes. This analysis reinforces the recognition that humid to semi-humid alluvial valleys constrain aeolian depositional architecture in ways distinct from open dryland environments (e.g., Langford, 1989; Liu and Coulthard, 2015; Zhou et al., 2014). For example, S2 alluvial ridge crests, which act as promontories to decrease and redirect surface wind vectors, were preferred points of deposition for up to $2 \mathrm{~m}$ of very fine sandy sediment $\left(\bar{x}_{\varphi}=4.24 \varphi\right)$. In this case, sedimentation was strongly influenced by the morphology of underlying alluvial topography, and perhaps vegetation, in addition to surface wind vectors and sediment availability.

\subsection{Regional Comparisons and Possible Paleoclimate Correlations}

Recent geochronological studies are yielding a growing inventory of $<12$ ka late Pleistocene through Holocene OSL and radiocarbon ages for aeolian landforms from a variety of geomorphic settings in the eastern U.S. (Campbell, Fisher, \& Goble, 2011; E. Hansen, Arbogast, Packman, \& Hansen, 2002; Z. Kilibarda, Venturelli, \& Goble, 2014; Krieg et al., 2004; Lutz, Wiles, Lowell, $\&$ Michaels, 2007). Most relevant to the current study are aeolian landforms such as dune fields located within alluvial valleys, paleochannels, or outwash/till plains (Arbogast et al., 2015; Blockland, 2013; Zoran Kilibarda \& Blockland, 2011; Miao et al., 2010; J E Rawling, Hanson, Young, \& Attig, 2008; Hong Wang et al., 2012). Dune fields are common in eastern U.S. alluvial settings, especially low-relief glaciated landscapes where dunes tend to reflect parabolic and sand sheet forms. Similar to Sandy Springs, individual dunes heights vary for eastern U.S. landforms but most are less than $15 \mathrm{~m}$ in height (Blockland, 2013: 27-32; Kilibarda and Blockland, 2011: 307; Miao et al., 2010: 764; Rawling et al., 2008: 495). 
Late Pleistocene through Holocene aeolian sedimentation predominately is interpreted as reactivation and resculpting of the upper sections of extant Pleistocene dunes (Blockland, 2013; Miao et al., 2010), an interpretation also favored for Sandy Springs. Similar to dunes at Sandy Springs, most $<12$ ka ages for aeolian landforms in the eastern U.S. are recovered from shallow $<2 \mathrm{~m}$ deposits, although early ages from depths between 5 and $7 \mathrm{~m}$ also are reported (Arbogast et al., 2015: 112; Kilibarda and Blockland, 2011: 316; Rawling et al., 2008: 498). At least one dune in Illinois, the Bill Farm site, has OSL ages suggesting construction entirely during the Holocene (Miao et al., 2010: 768). At Sandy Springs, OSL ages suggest that significant sections of S2 sand mantles and the compound barchan dune also were constructed primarily during the Holocene (see Table 4-1 and Fig. 4-4). Although buried surfaces (i.e., Ab horizons) were not identified in aeolian contexts at Sandy Springs, their occurrence at other sites in Illinois, Ohio, and Wisconsin (Blockland, 2013; Miao et al., 2010; J E Rawling et al., 2008; Hong Wang et al., 2012) suggests aeolian depositional hiatuses were common over the last 12 ka years in the eastern U.S.

The construction of late Pleistocene through Holcoene aeolian landforms in the eastern U.S. has been attributed to a combination of factors including environmental change, notably increased aridity (Campbell et al., 2011; Zoran Kilibarda \& Blockland, 2011; Miao et al., 2010), increased sediment supply (Arbogast et al., 2015), and increased sediment availability through

groundwater drawdown and deflation (Miao et al., 2010; J E Rawling et al., 2008). Although not widely studied, local disturbances also may be responsible for site-specific reactivations such as animal overgrazing, wildfires, and Native American land-use practices (Miao et al., 2010: 770). Although local wind fetch and sediment availability are interpreted as important factors for initiating aeolian processes at Sandy Springs, OSL and AMS radiocarbon dating and internal dune architecture also suggest at least two possible linkages of local landscape instability to panregional Holocene paleoclimate events.

First, the OSL age of 8.2 ka from S3 deposits indicates early Holocene deposition of finetextured coversands on this high surface. Although based on a single age, we suggest the possibility that this depositional event is related to the $8.2 \mathrm{ka}$ paleoclimate event, or North Atlantic Bond Event 5 (Bond et al., 1997). The $8.2 \mathrm{ka}$ event reflects an abrupt centennial-scale cooling and drying episode characterized by increased windiness that appears related to North Atlantic sea-surface cooling due to the final collapse of the Laurentide Ice Sheet (e.g., Alley et al., 1997; Alley and Ágústsdóttir, 2005; Bond et al., 1997; Ellison et al., 2006; Hu et al., 1999; Kobashi et al., 2007; Li et al., 2007; Shuman et al., 2002; Yu and Eicher, 2001). In North America specifically, increased dust frequencies in lacustrine sediments have been cited as evidence for increased aeolian activity across the continental U.S. at this time (e.g., Dean et al., 2002; Fritz et al., 2001; Hu et al., 1999; Lutz et al., 2007; Tornqvist et al., 2004). Potential linkages to the $8.2 \mathrm{ka}$ event have been suggested from two additional Ohio sites. Coring of lacustrine sediments at northcentral Ohio's Brown's Lake reveal two distinct, organic-poor silt beds dated between 8.9 and $8.2 \mathrm{ka}$ (Lutz et al., 2007), which is interpreted as evidence for increased aeolian activity. Aeolian reactivation of late Pleistocene beach deposits and sand dunes in northwestern Ohio also has been documented beginning $\sim 8.8 \mathrm{ka}$ and may reflect an early onset of the $8.2 \mathrm{ka}$ event (Campbell et al., 2011).

A second possible paleoclimate link includes evidence of likely synchronous reworking of sediments on three different Sandy Springs landforms at 4.5 ka (BG 4160, BG 4161, BG 4162, 
see Table 4-1). One of these ages derives from the longitudinal dune characterized by high-angle $\left(>30^{\circ}\right)$ planar cross-beds indicative of dune reactivation and slipface grain flow. A second age is from the limb section of the compound barchan-like dune and may reflect either dune/limb construction or subsequent reactivation. The final age derives from a gully cut in the S2 deflated basin that exposed low-angle cross-stratification of reworked outwash. The significant erosion, transport, and deposition at $\sim 4.5 \mathrm{ka}$ corresponds to a period of lower lake levels and increased aridity in northeastern U.S. centered at $\sim 4.4 \mathrm{ka}$ (Y.-X. Li et al., 2007) and severe drought at $\sim 4.2$ ka for significant portions of the Northern Hemisphere (e.g., Alley et al., 2003; Booth et al., 2005; Booth et al., 2004; Dean, 1997; Staubwasser et al., 2003). Drought conditions like those reported for the $4.2 \mathrm{ka}$ event are known to result in severe landscape modification and initiation of aeolian processes such as dune reactivation across portions of North America (e.g., Forman et al., 2001; Mason et al., 2004; Mason et al., 1997) and a similar set of processes may have been occurring throughout the upper Ohio River Valley during this time.

\subsection{Conclusions}

This research has documented various aspects of a dynamic late Quaternary alluvial landscape within the upper Ohio Valley. Geomorphic, pedogenic, stratigraphic, and sedimentary observations, and chronostratigraphic data, demonstrate that a complex interplay of aeolianalluvial processes sculpted the Sandy Springs landscape since at least $17 \mathrm{ka}$. Aeolian sedimentation as dune sand on S2 (aeolian unit I) and fine-textured coversands (aeolian unit II) on S3 was active between 11 and $1.4 \mathrm{ka}$. This finding indicates that even for Pleistocene landforms in the unglaciated upper Ohio Valley, significant aeolian-fluvial erosion and sedimentation occurred episodically throughout the Holocene. The dating of sediment units at Sandy Springs suggest that the 4.2 ka paleoclimate event, and perhaps the earlier 8.2 event, played significant roles in development of landscapes in the upper Ohio Valley into the Holocene. Although initial LiDAR and soils data suggested a near continuous distribution of aeolian dunes surrounding the S2 deflation basin, this study has revealed a more complex interaction of fluvial and aeolian processes with the underlying alluvial topography. Whereas hummocky terrain proximal to S3 at Sandy Springs reflects relict dunes consisting of crossbedded sands of varied thickness, aeolian landforms to the west of US 52 on the S2 tread are not true dunes structures, but instead reflect sand-mantled alluvial ridge crests. This finding reinforces the understanding that more humid alluvial valleys constrain aeolian-fluvial processes in ways distinct from more open dryland settings such as ergs or coastal plains.

\section{Acknowledgements}

Geochronology and micromorphology work is supported by a 2016 NASA West Virginia Space Grant Consortium Graduate Research Fellowship. We thank various individuals for providing project assistance including Steve Baker of USDA-NRCS; Mike Angle, Frank Fugitt, and Nathan Erber of the Ohio Geological Survey; James Thompson and Kathy Benison of West Virginia University; Chris Bedel of the Edge of Appalachia Preserve System; and Nancy Stranahan of the Arc of Appalachia. The following also assisted in field and laboratory efforts: Francesca Basil, Andrew Braun, Nick Dadamo, Sara DeAloia, Nancy Gostic, Zach Haidar, Heather Jewel, Matt Koerner, Miles Reed, Emily Swaney, Katie Wasley, and Joey Zampayo. Special thanks to the Adams, Rutledge, and Whisman families for providing property access. 
Chapter 4. Journal Article Supplementary Material

\section{S1. Additional Graphics}

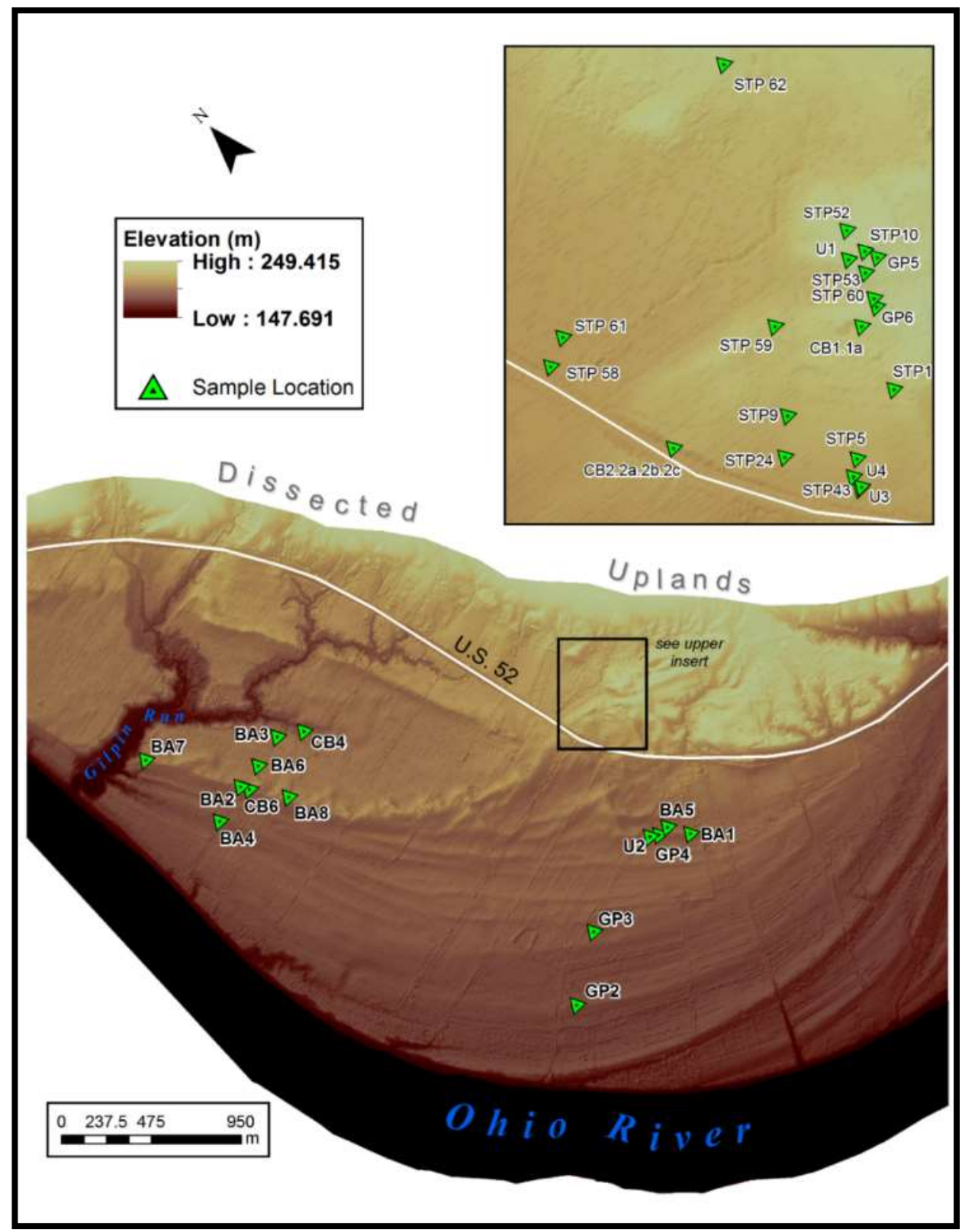

Fig. S23. Distribution of sample locations across Sandy Springs $(B A=$ bucket auger; $\mathrm{CB}=$ cutbank exposure; $\mathrm{GP}=$ Giddings probe; $\mathrm{STP}$ or $\mathrm{U}=$ soil test pit). 


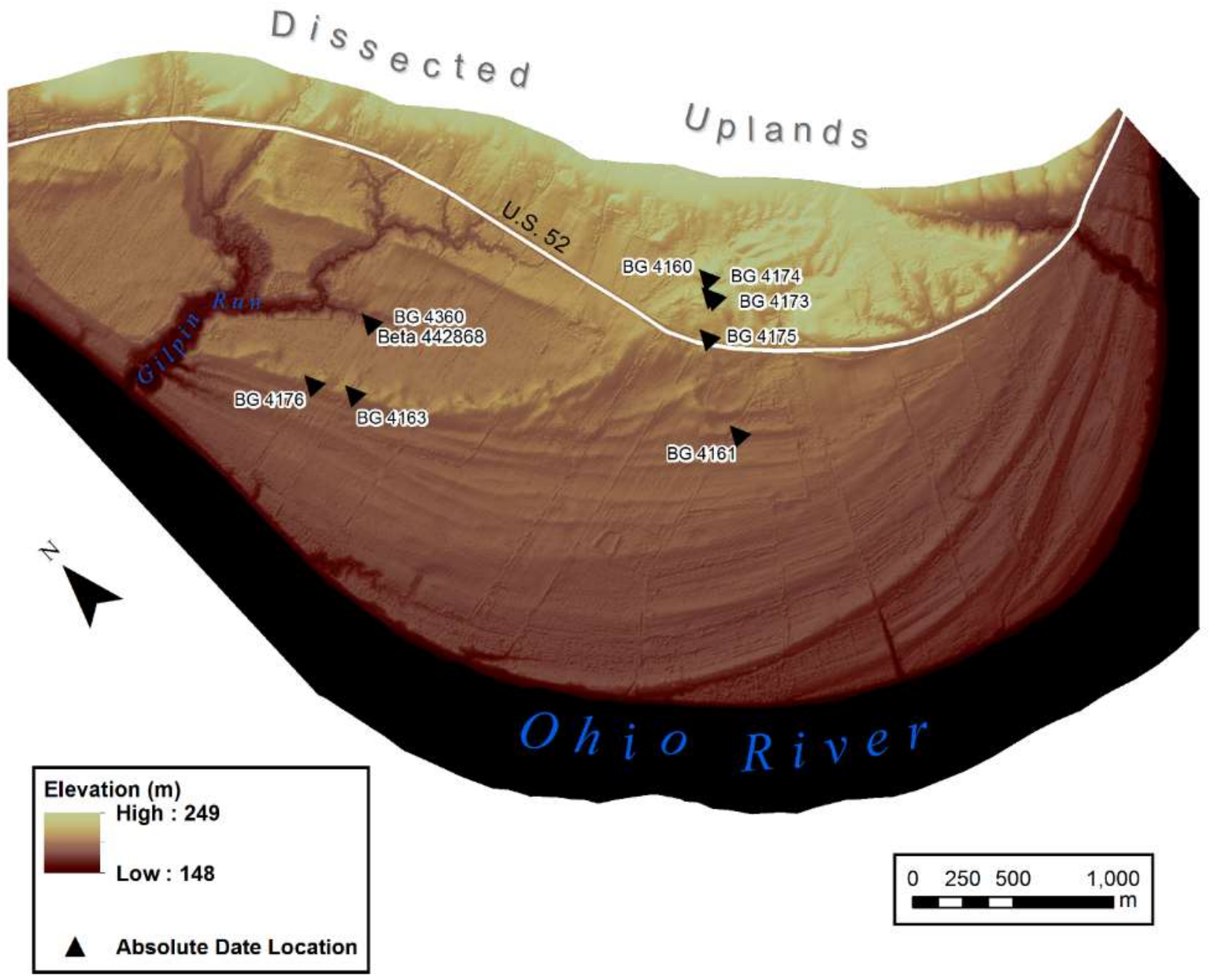

Fig. S24. OSL and AMS sample locations. "BG" reflect OSL sample locations; whereas "Beta" represent AMS sample location. 
S2. Results of OSL dating of eight sediments samples from the Sandy Springs Project, Adams County, Ohio

Steven J. Forman

Eight sediment samples were submitted to the Geoluminescence Dating Research Lab at Baylor University for OSL dating (see main text Table 4-1). Single aliquot regeneration (SAR) protocols (Murray \& Wintle, 2003) were used for OSL dating to estimate the apparent equivalent dose of the $425-355,425-500,355-250$, and 63-44 $\mu \mathrm{m}$ quartz fractions for 28 to 61 separate aliquots (see main text Table 4-1). Each aliquot contained approximately 100 quartz grains corresponding to a $1.5 \mathrm{~mm}$ to $2.0 \mathrm{~mm}$ circular diameter of grains adhered (with silicon) to a $1 \mathrm{~cm}$ diameter circular aluminum disc. Analyzed sands were mineralogically mature with $\mathrm{SiO} 2$ content of $70 \%$ to $90 \%$ of the non-carbonate fraction and are predominantly $(>80 \%)$ well-sorted quartz grains. The quartz fraction was isolated by density separations using the heavy liquid $\mathrm{Na}$-polytungstate, and a 40-min immersion in HF (40\%) was applied to etch the outer $\sim 10 \mu \mathrm{m}$ of grains, which is affected by alpha radiation (Mejdahl \& Christiansen, 1994). Quartz grains were rinsed finally in $\mathrm{HCl}(10 \%)$ to remove insoluble fluorides. Quartz separates purity was evaluated by petrography and point counting of a representative aliquot. Samples that showed $>1 \%$ of non-quartz minerals were retreated with HF and rechecked petrographically. The purity of quartz separates was tested by exposing aliquots to infrared excitation ( $1.08 \mathrm{w}$ from a laser diode at $845 \pm 4 \mathrm{~nm}$ ), which preferentially excites feldspar minerals. Samples measured showed weak emissions $(<200$ counts/s), at or close to background counts with infrared excitation, and ratio of emissions from blue to infrared excitation of $>20$, indicating a spectrally pure quartz extract (Duller, BøtterJensen, \& Murray, 2003).

A series of experiments was performed to evaluate the effect of preheating at 200, 220, 240 and $260{ }^{\circ} \mathrm{C}$ on isolating the most robust time-sensitive emissions and thermal transfer of the regenerative signal prior to the application of SAR dating protocols (see Murray \& Wintle, 2003). These experiments entailed giving a known dose (20 Gy) and evaluating which preheat resulted in recovery of this dose. There was concordance with the known dose (20 Gy) for preheat temperatures above $220{ }^{\circ} \mathrm{C}$ with an initial preheat temperature used of $220{ }^{\circ} \mathrm{C}$ for $40 \mathrm{~s}$ in the SAR protocols. A "cut heat" at $220^{\circ} \mathrm{C}$ for $40 \mathrm{~s}$ was applied prior to the measurement of the test dose and a final heating at $260{ }^{\circ} \mathrm{C}$ for $40 \mathrm{~s}$ was applied to minimize carryover of luminescence to the succession of regenerative doses (see main text Table 4-1). A test for dose reproducibility was also performed following procedures of Murray and Wintle (2003) with the initial and final regenerative dose of $\sim 16$ Gy yielding concordant luminescence responses (at one-sigma error).

Typical OSL shine-down curves for quartz grains are shown in Fig. S25. The curve shapes show that OSL signal is probably dominated by a fast component, with the OSL emission decreasing by 90 to $95 \%$ during the first 4 seconds of stimulation. The regenerative growth curves are modeled by using the exponential plus linear form. For many aliquots the regenerative growth curves (Fig. S25) show that (1) the recuperation is close to zero; (2) the recycling ratio is consistent with unity at $1 \sigma$; (3) the natural Lx/Tx ratio is within $20 \%$ of the saturated level. Most aliquots were removed because of unacceptable recycling ratio and De values at or close to saturation with errors of $>10 \%$. Error analysis for equivalent dose calculations assumed 
measurement error of $1 \%$ and Monte Carlo simulation repeats of 2000 . Recuperation is lower than 3\% for all samples, which indicates insignificant charge transfer during the measurements. These favorable luminescence characteristics for a majority of aliquots indicate that credible equivalent dose values for these sediments can be determined by the SAR protocol.

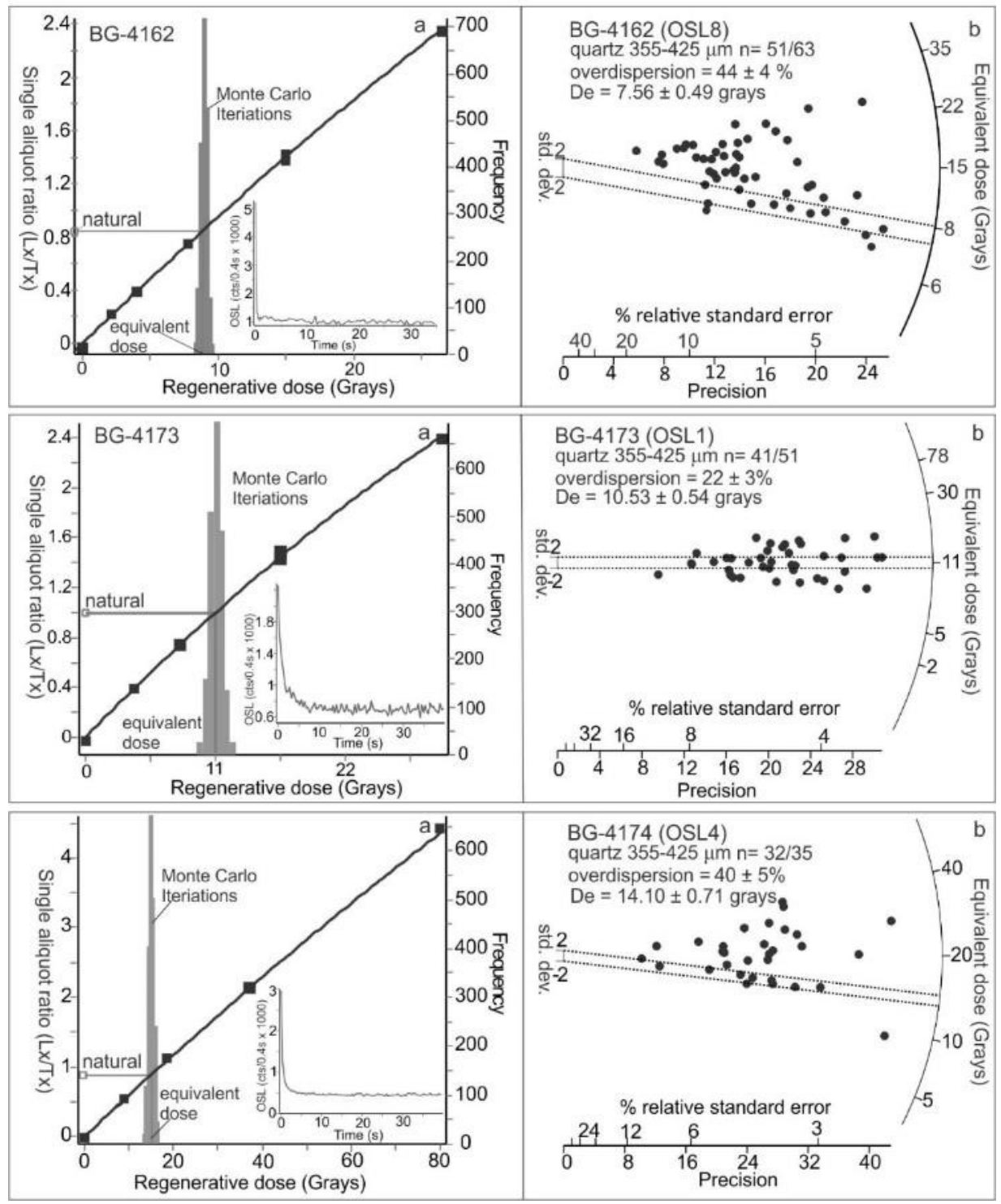

Fig. S25. Representative regenerative dose growth curves, with inset representative natural shine down curve, and radial plots of equivalent dose values on small aliquots (2-mm plate of 44-500 $\mu \mathrm{m}$ quartz fraction grains, see main text Table 1).

Calculation of equivalent dose by SAR protocols was accomplished for 23 to 60 aliquots. Equivalent dose distributions were usually log normal and the scatter in the data is quantified with overdispersion values (see main text Table 4-1; Fig. S25). An overdispersion percentage of a De distribution is an estimate of the relative standard deviation from a central De value in 
context of a statistical estimate of errors (Galbraith \& Roberts, 2012; Galbraith et al., 1999). A zero overdispersion percentage indicates high internal consistency in De values with $95 \%$ of the De values within $2 \sigma$ errors. Overdispersion values $\leq 20 \%$ are routinely assessed for quartz grains that are well solar reset, like aeolian sands (e.g., Wright et al., 2011) and this value is considered a threshold metric for calculation of a De value using the central age model of Galbraith et al. (1999). Overdispersion values $>20 \%$ indicate mixing or grains of various ages or partial solar resetting of grains; the minimum age model (three parameters) may be an appropriate statistical treatment for such data and effectively weights for the youngest De distribution. However, some studies have concluded that overdispersion values between 20 and $32 \%$ may reflect a signal De population, particularly if the De distribution is symmetrical, with the dispersion related to variability associated with micro-dosimetry and/or sedimentary processes (e.g., Arnold \& Roberts, 2009). We consider overdispersion values $>20 \%$ (at one sigma limits) to indicate post depositional mixing of grains of various ages, partial solar resetting of grains or complex microdosimetry; the Finite Mixture Model (FMM) is an appropriate statistical treatment for such data (Galbraith \& Green, 1990), and this model was used for quartz extracts that yielded overdispersion values of $25 \%$ (Table S11).

Table S11. Single Aliquot Regeneration Protocols

\begin{tabular}{|c|l|}
\hline Step & Treatment \\
\hline 1 & Natural dose or give beta dose \\
\hline 2 & Preheat $240^{\circ} \mathrm{C}$ for $10 \mathrm{~s}$ \\
\hline 3 & Stimulate with blue light $(470 \mathrm{~nm})$ for $40 \mathrm{~s}$ at $125^{\circ} \mathrm{C}$ \\
\hline 4 & Give beta test dose $(6.6$ Gray) \\
\hline 5 & Preheat $240{ }^{\circ} \mathrm{C}$ for $10 \mathrm{~s}$ \\
\hline 6 & Stimulate with blue light $(470 \mathrm{~nm})$ for $40 \mathrm{~s}$ at $125^{\circ} \mathrm{C}$ \\
\hline 7 & Stimulate with blue light for $40 \mathrm{~s}$ at $280^{\circ} \mathrm{C}$ \\
\hline 8 & Return to step 1 \\
\hline
\end{tabular}

The environmental dose rate is critical measurement for calculating a luminescence age, which is an estimate of the exposure of quartz grains to ionizing radiation from the decay of the $U$ and $T h$ series, $40 \mathrm{~K}$, and cosmic sources during the burial period. The $\mathrm{U}$, Th and $\mathrm{K}$ concentrations are determined by inductively coupled plasma mass spectrometry by Activation Laboratory LTD, Ontario, Canada. The beta and gamma doses were adjusted according to grain diameter to compensate for mass attenuation for the dose rate (Fain et al., 1999). Beta and gamma attenuation coefficients for 355 to $425 \mu \mathrm{m}$ are 0.800 and 0.997 , respectively. The $\mathrm{U}$, Th and $\mathrm{K} 2 \mathrm{O}$ content was determined for the bulk sediment to calculate the dose rate. A cosmic ray component, considering location, elevation and depth of strata sampled is between 0.16 and 0.20 $\mathrm{mGy} / \mathrm{yr}$ and is included in the estimated dose rate (Prescott \& Hutton, 1994). There is uncertainty in assessing the moisture content of a sample during the burial period. We estimated moisture contents from present values, particle size characteristics and in reference to the water table. 
Chapter 4. Non-journal Supplemental Information: Micromorphological Analysis

Micromorphological analysis of sediments was proposed as a method to better understand the depositional and environmental history of the Sandy Springs landscape (Purtill, 2015).

Micromorphology involves the examination of sediment thin-sections to identify such properties as the presence of illuviated clay, clay aggregates, elements of fabric, organic residues, etc.

(FitzPatrick, 1984; Stoops, 2003, 2010; Vepraskas \& Wilson, 2008). Due to spatial limitations for journal submission, the full results of the micromorphological analysis were not included in the main article text. Since this information could be useful in reconstruction various formational aspects of alluvial dunes and may be the basis of a future article in its own right, micromorphological results are provided below.

Twelve 5 x $7.5 \mathrm{~cm}$ thin-sections, prepared at National Petrographic Services, Inc., Rosenberg, Texas, were analyzed following protocols established by Stoops (2003). Slides are from a variety of contexts, primarily from aeolian and alluvial settings. All 12 slides are scanned with a polarizing microscope under plane polarized light (PPL), cross-polarized light (XPL), and oblique incident light (OIL). Observations regarding each slides microstructure and porosity, groundmass, organic material, and pedofeatures are recorded on standardized forms.

Additionally, four slides are subject to detailed compositional analysis of mineral grains through point counting within a $1 \mathrm{~mm}$ (horizontal) by $2 \mathrm{~mm}$ (vertical) grid system similar to the procedure suggested by FitzPatrick (1984: 104-106). This approach provides comprehensive slide coverage yielding between 800 and 1000 potential mineral identifications per thin-section. Longest axial length and roundness, slightly modified from Powers (1953), are recorded for identified grains. When voids are encountered at observation points, the closest grain within 0.5 $\mathrm{mm}$ is selected for identification.

Quantitative point counting is conducted on four of these slides (MS1, MS4, MS5, and MS11) to provide detailed compositional data (Table S12). In all contexts, quartz dominates the identifiable assemblage at $>85$ percent. Chert is common in aeolian contexts accounting for up to seven percent of identified grains in thin-section. Muscovite is rare in medium-sand textured aeolian units but more common $(\sim 1-3 \%)$ in very fine- to fine-textured sediment interpreted as aeolian (MS11) and possibly fluvial (MS9).

Overall, grain morphologies are submature, primarily sub-rounded particles of low sphericity. Only seven percent of grains interpreted as aeolian are well-rounded with high sphericity, a morphology traditionally associated with far-traveled aeolian dune sands (Pye and Tsoar, 2009: 82). Aeolian thin-section sediments exhibit apedal, coarse monic, single-grain microstructures. Very fine to fine sand textured micro-laminations within a close porphyric $\mathrm{c} / \mathrm{f}$ related distribution pattern also is noted at $3.1 \mathrm{~m}$ of the knob landform (MS11). 
Distinct clay aggregates or pellets represent $\sim 10$ percent of the grains identified in Sandy Springs thin-sections from deposits interpreted as aeolian (MS1-MS3; MS5-MS7). In contrast, clay aggregates are rare $(<1 \%)$ in non-aeolian contexts. Aggregates have been commonly reported in central and eastern U.S. dune and loess deposits (e.g., Kilibarda and Blockland, 2011: 309-310; Mason et al., 2003). Pelletization occurs under arid or seasonally dry conditions where wind erosion detaches and entrains the edges of mud curls or salt-mud efflorescences (Pye, 1987: 27; Pye and Tsoar, 2009: 93; Shaw and Bryant, 2011: 392). The presence of intact aggregates in minimally weathered $\mathrm{C}$ horizons of Peoria Loess in Nebraska suggest to some that pellets represent transported sediments and not pedogenic features (Mason et al., 2003: 385). In contrast, similar aggregates reported from A horizons in grassland soils are thought pedogenically developed (e.g., Sanborn and Pawluk, 1989).

At Sandy Springs, clay aggregates are tempered predominately with silt-sized quartz grains although muscovite grains occur less frequently (see Fig. S26C). Aggregates typically exhibit clear subrounded boundaries of low sphericity. Evidence of particle disaggregation and coalescing, reported as common by some (Mason et al., 2003: 383), is infrequent at Sandy Springs. Secondary (hydro)oxides impregnate some aggregates. Clay aggregates have an effective particle size between medium and coarse sand $(\bar{x}=0.6 \mathrm{~mm}$, or $0.7 \varphi)$. Axial grain length of clay aggregates is $0.47 \mathrm{~mm}$ but if corrected for thin-section measurement error (Stoops, 2003: 12) have a mean apparent grain size increases to $0.6 \mathrm{~mm}$. Clay aggregate axial length is 14 percent longer than quartz grains in aeolian deposits. The presence of abundant, intact, clay aggregates from minimally weathered C horizon dune contexts at Sandy Springs are interpreted as the result of aeolian not pedogenic processes. This interpretation is based on the observation that most clay aggregates in thin-section have sharp boundaries and appear anorthic which fails to suggest in-situ pedogenic development (Stoops, 2003).

Finally, micromorphological results also inform on the structure and origin of lamellae (Bt horizon) and inter-lamellae (E horizon) zones within the barchan-like dune (MS5 and MS6). Analysis revealed well-oriented limpid clay domains that coat and bridge individual grains and fill pore space within lamellae (see Fig. S26D-E). In XPL, lamellae clay domains are birefringent and exhibit sharp extinction lines suggestive of illuvial translocation (Fedoroff, 1974; Stephen, 1960; Stoops, 2003: 19) and are interpreted as secondary or pedogenic structures (J. Elmo Rawling, 2000). MS5 and MS6 also reveal clay aggregates as roughly twice as abundant in interlamellae zones than lamellae bands. This abundance likely indicates the weathering of clay aggregates is the primary source of clay found within lamellae and dunes as suggested by others (e.g., Bowler, 1973; Kilibarda et al., 2008). 
Table S12. Point counting compositional data of micromorphological thin-section samples (Facies defined in main text)

\begin{tabular}{|l|l|l|r|r|r|r|r|r|r|}
\hline Facies & $\begin{array}{l}\text { Geomorphic } \\
\text { Context }\end{array}$ & $\begin{array}{l}\text { Micromorphology } \\
\text { Sample Number } \\
\text { (depth) }\end{array}$ & Q & C & F & M & B/H & Q:CA & Q:F \\
\hline Aeolian unit I & Sand Dune & $\begin{array}{l}\text { MS1 }(2.65 \mathrm{~m}), \text { MS5 } \\
(0.9 \mathrm{~m})\end{array}$ & $91 \%$ & $6 \%$ & $<1 \%$ & $<1 \%$ & $<1 \%$ & $7.4: 1$ & $36.2: 1$ \\
\hline Aeolian unit II & Knob & MS11 $(3.1 \mathrm{~m})$ & $94 \%$ & $\begin{array}{r}<1 \\
\%\end{array}$ & $1 \%$ & $3 \%$ & $1 \%$ & $665: 1$ & $83: 1$ \\
\hline Indeterminate & Knob & MS4 $(0.42 \mathrm{~m})$ & $96 \%$ & $\begin{array}{r}1.5 \\
\%\end{array}$ & $1 \%$ & $<1 \%$ & $<1 \%$ & $120: 1$ & $90: 1$ \\
\hline
\end{tabular}

$\mathrm{Q}=$ quartz; $\mathrm{C}=$ chert; $\mathrm{F}$ = feldspar; $\mathrm{M}=$ muscovite; $\mathrm{B}=$ biotite; $\mathrm{H}=$ amphibole/hornblende; $\mathrm{Q}: \mathrm{CA}$ = quartz:clay aggregate ratio; $\mathrm{Q}: \mathrm{F}=$ quartz:feldspar ratio.

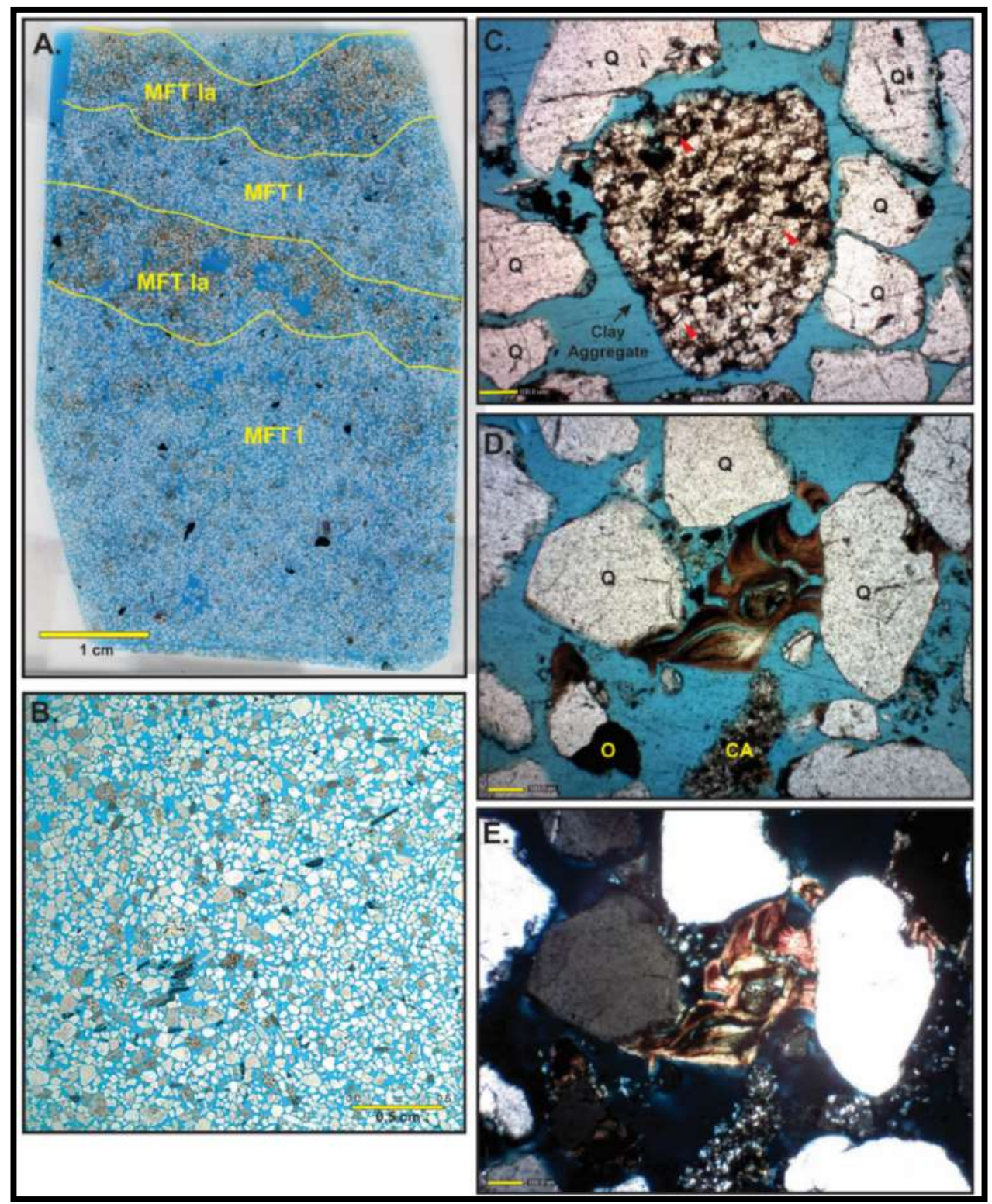

Fig. S26. Representative photomicrographs and scans from Sandy Springs thin-sections with pore space dyed blue. A. Scan of MS5 thin-section slide from $0.90 \mathrm{~m}$ in a 3E\&Bt horizon within 
compound barchan-like dune limb (U2). Slide contains two microfacies types (MFT) representing inter-lamellae (MFT I) and lamellae (MFT Ia) zones. B. Photomicrograph in PPL showing section of MS1 at $2.65 \mathrm{~m}, 5 \mathrm{C}$ horizon, from longitudinal dune (CB1a). Brown, "dirty" grains tempered with silt-sized quartz primarily represent clay aggregates. Black, rod-shaped (hydro)oxides show weak parallel alignment matching macro-observations of bedding planes with a true dip orientation of $33^{\circ} \mathrm{NNE}$. C. Isolated view of clay aggregate grain identified in MS1 (see B. for context description). Red arrows denote silt-sized muscovite grains within aggregate. Impregnative black (hydro)oxide formation within aggregate also evident. PPL (D.) and XPL (E.) views of well-oriented illuviated clay domains linking quartz grains in compound barchan limb (U2) at $0.9 \mathrm{~m}$ in a $3 \mathrm{E} \& \mathrm{Bt}$ horizon. Note sharp extinction lines for clay domains in XPL. $\mathrm{Q}=$ quartz; $\mathrm{CA}=$ clay aggregate; $\mathrm{O}=$ (hydro)oxide. 


\section{CHAPTER 5. CONCLUDING REFLECTIONS}

Recent literature has made it is increasingly clear that many traditional models of eastern U.S. Paleoindian chronology, settlement, economics, land-use behavior, and subsistence, require careful reevaluation (e.g., Anderson, 2013; Anderson et al., 2015; Ellis, 2011; Eren et al., 2015; Holliday \& Miller, 2014; Meltzer \& Holliday, 2010; Speth et al., 2013; Waters \& Stafford, 2007). Many current models rely heavily on data from poorly documented sites or from hypothesized environmental co-associations often based on qualitative observations. Recent trends in archaeological research include calls to reinvestigate classic eastern U.S. Paleoindian sites to determine what new insights are available (see Gingerich, 2013b, 2018).

This dissertation revisits the issue of Paleoindian occupation and land-use behavior in southern Ohio and the upper Ohio River Valley in the eastern U.S. The approach of this research was multi-scalar in nature and included investigating the problem at both the regional and local levels. It is argued that this multi-scalar approach is essential at revealing the various aspects of Paleoindian land-use behavior, including a better understanding of the physical environment within which they interacted. The multi-scalar approach also heeds Michael Waters' words that "good archaeology cannot be done without good geology" (Waters, 2004) as most archaeological geology is conducted at a more local scalar level. In following this approach, this research has built upon interpretations offered by Seeman \& Prufer (1982) and has provided new insights into Paleoindian lifeways. Both previously proposed and newly generated hypotheses of Paleoindian land use in southern Ohio were explored through a GIS and statistical modeling approach. A reevaluation of the geomorphological and archaeological history of the Sandy Springs landscape also was conducted. Below is a list of the primary research findings:

- Regional level:

- Study results broadly indicate that Paleoindians did not practice a uniform landuse strategy in the upper Ohio Valley but instead adapted strategies to fit local conditions.

- A potentially distinct Paleoindian land-use strategy was identified for the Interior Low Plateaus province of Ohio. This strategy may reflect strengthening post-11 ka interaction between groups occupying the Cumberland, Tennessee, and upper Ohio drainages. This strategy also may indicate a preference for prairie environments.

- The combined modeling of Paleoindian, Early Archaic, and Late Archaic site distributions provides a method to assess the potential of collector bias. Results indicate that current population densities are positively correlated with archaeological site densities of all periods and demonstrates that collector bias in site distribution is not restricted to Paleoindian assemblages. Cross-temporal approaches provide a means to mitigate the influence of such bias in future studies. 
- Local, or site level:

- Sandy Springs represents one of the largest Paleoindian sites in the upper Ohio River Valley. Geomorphological and geochronological research indicates that Sandy Springs is located on a late Quaternary landscape shaped by aeolianalluvial processes that have the potential to cover archaeological deposits including Paleoindian components.

- Reanalysis of artifacts from the 1976 survey and the results of new survey suggest that Paleoindian occupation of the landscape occurred in several spatial loci across the landscape.

- The previous claim of saline springs at the Paleoindian Sandy Springs site by Roger Cunningham was tested through electrical conductivity and $\mathrm{pH}$ analysis. Cunningham and others have asserted that saline groundwater was a draw for Paleoindian groups which explained why Sandy Springs was so intensively occupied. Results of this research indicate that groundwater at Sandy Spring is low in salinity thus questioning the accuracy of the existing settlement and subsistence model forwarded for the site.

- Alternative explanations of Paleoindian occupancy at Sandy Springs were advanced including the presence of a rare xeric ecosystem and its location along an early trail system connecting the Cumberland, Tennessee, and Ohio river valleys.

- The broader impacts of the geomorphological research potentially demonstrate that many upper Ohio Valley Pleistocene landforms are capped by Holocene aeolian sediments. This realization has important implications for the potential of archaeological site burial in the Ohio Valley.

- A multi-tiered statistical and graphical approach to particle-size analysis was successful at discriminating depositional environments at Sandy Springs.

- Research also suggests that paleoclimate, including the well-documented 8.2 and $4.2 \mathrm{ka}$ events, may have played a significant role in shaping landscapes in the upper Ohio Valley throughout the late Pleistocene and the Holocene.

Although this dissertation addressed various aspects of Paleoindian occupation and land-use behavior and methodological approaches to modeling, assessing collector bias, and determination of depositional environments, additional research should build upon these results. The following thoughts or comments are offered to help build a future foundation:

1. The approach of modeling archaeological and environmental variables should be pursued and refined; especially issues related to variable resolution, where variables modeled at a particular resolution may be mismatched to the scale of the cultural behavior under study (Harvey, 1968; Lam \& Quattrochi, 1992; K. H. Stone, 1972).

2. More attention also should focus on identifying 'cultural' variables (G. R. Lock \& Harris, 2006) that may be influential in determining site location during the Paleoindian period and beyond. 
3. This research identified that collector bias is indeed an issue for archaeological site distribution studies as they related to modern population densities. This bias is not restricted to Paleoindian sites, but appears to extend equally to all time periods considered in this study. It was demonstrated that a cross-temporal approach is an effective way to evaluate the validity of observed site patterns. Another, perhaps more promising approach, would be to weight archaeological and random data points by modern population density as a means for controlling for the uneven distribution of 'potential collectors' across the modern landscape.

4. Relatedly, this dataset may have been biased to a large degree by the urban presence of the city of Cincinnati and surrounding suburbs in Hamilton County. The fact that many archaeological sites are not recorded in the footprint of Cincinnati undoubtedly is related to urban sprawl and not prehistoric population distributions. Future studies for southern Ohio should consider excluding Hamilton from any datasets to help control for this form of bias.

5. The results of the geomorphological and geochronological analysis at Sandy Springs demonstrates that late Pleistocene to Holocene aeolian sediments blanket many landforms in the upper Ohio Valley. In such contexts, the potential for these sediments cover unidentified archaeological resources has not been fully appreciated in the archaeological community (see Purtill, 2017). Future research should focus on identifying these landforms and evaluating their potential for site burial. The particle-size and PCA methodology developed herein has considerable potential for identifying aeolian sediments and should be refined and applied to other environmental settings.

6. Finally, the development of more accurate models of Paleoindian occupation and landuse behavior is inextricably linked to the number of known Paleoindian archaeological sites with good provenience information. Continued efforts should encourage site identification in southern Ohio and elsewhere. Newly discovered sites must be inventoried within the state OAI system so future studies can build upon or refine the results of this dissertation. 


\section{REFERENCES}

Abella, S. R., \& Jaeger, J. F. (2004). Ecology and Eastern Prickly Pear Cactus (Opuntia humifusa) in Oak Openings Preserve, Northwestern Ohio. The Michigan Botanist, 43, 1-11.

Aden, D. J., Pavey, R. R., Jones, M. D., \& Angle, M. P. (2012). Surfacial Geology of the Defiance 30 X 60-minute Quadrangle in Ohio (1:100,000 scale). Ohio Department of Natural Resources, Division of Geological Survey Map SG-2-DEF. Columbus.

Aitken, M. J. (1998). An introduction to optical dating: The dating of Quaternary sediments by the use of photon-stimulated luminescence. Oxford science publications. https://doi.org/10.1002/(SICI)1520-6548(200001)15:1<81::AID-GEA5>3.3.CO;2-Y

Al-Busaidi, A. S., \& Cookson, P. (2003). Salinity-pH Relationships in Calcareous Soils. Agricultural and Marine Sciences, 8(1), 41-46.

Alley, R. B., \& Ágústsdóttir, A. M. (2005). The 8k Event: Cause and Consequences of a Major Holocene Abrupt Climate Change. Quaternary Science Reviews, 24(10-11), 1123-1149. https://doi.org/10.1016/j.quascirev.2004.12.004

Alley, R. B., Marotzke, J., Nordhaus, W. D., Overpeck, J. T., Peteet, D. M., Pielke, R. A., ... Trumbore, S. (2003). Abrupt Climate Change. Science (New York, N.Y.), 299(5615), 200510. https://doi.org/10.1126/science.1081056

Alley, R. B., Mayewski, P. A., Sowers, T., Stuiver, M., Taylor, K. C., \& Clark, P. U. (1997). Holocene Climatic Instability: A Prominent, Widespread Event 8200 yr Ago. Geology, 25(6), 483-486. https://doi.org/10.1130/0091-7613(1997)025<0483:HCIAPW>2.3.CO;2

Anderson, D. G. (1990). The Paleoindian Colonization of Eastern North America. Research in Economic Anthropology Supplement, 5, 163-216.

Anderson, D. G. (1995). Paleoindian Interaction Networks in the Eastern Woodlands. In M. S. Nassaney \& K. E. Sassaman (Eds.), Native American Interaction: Multiscalar Analyses and Interpretations in the Eastern Woodlands (pp. 1-26). Knoxville: University of Tennessee Press.

Anderson, D. G. (1996). Models of Paleoindian and Early Archaic Settlement in the Lower Southeast. In D. G. Anderson \& K. E. Sassaman (Eds.), The Paleoindian and Early Archaic Southeast (pp. 29-57). Tuscloosa: University of Alabama Press.

Anderson, D. G. (2013). Paleoindian Archaeology in Eastern North America: Current Approaches and Future Directions. In J. A. M. Gingerich (Ed.), In the Eastern Fluted Point Tradition (pp. 371-404). Salt Lake City: The University of Utah Press.

Anderson, D. G., \& Faught, M. K. (1998). The Distribution of Fluted Paleoindian Projectile Points: Update 1998. Archaeology of Eastern North America.

Anderson, D. G., \& Faught, M. K. (2000). Palaeoindian artefact distributions: evidence and implications. Antiquity, 74, 507-513.

Anderson, D. G., \& Gillam, J. C. (2000). Paleoindian Colonization of the Americas: Implications from an Examination of Physiography, Demography, and Artifact Distribution. American Antiquity, 65(1), 43-66.

Anderson, D. G., Smallwood, A. M., \& Miller, D. S. (2015). Pleistocene Human Settlement in the Southeastern United States: Current Evidence and Future Directions. PaleoAmerica, 1(1), 7-51.

Arbogast, A. F., Luehmann, M. D., Miller, B. A., Wernette, P. A., Adams, K. M., Waha, J. D., ... Young, A. R. (2015). Late-Pleistocene Paleowinds and Aeolian Sand Mobilization in North-central Lower Michigan. Aeolian Research, 16, 109-116. 
https://doi.org/10.1016/j.aeolia.2014.08.006

Arbogast, A. F., Luehmann, M. D., William Monaghan, G., Lovis, W. A., \& Wang, H. (2017). Paleoenvironmental and geomorphic significance of bluff-top dunes along the Au Sable River in Northeastern Lower Michigan, USA. Geomorphology, 297, 112-121. https://doi.org/10.1016/j.geomorph.2017.09.017

Arnold, L. J., \& Roberts, R. G. (2009). Stochastic modelling of multi-grain equivalent dose (De) distributions: Implications for OSL dating of sediment mixtures. Quaternary Geochronology, 4(3), 204-230. https://doi.org/10.1016/j.quageo.2008.12.001

Bagnold, R. A. (1941). The Physics of Blown Sand and Desert Dunes. London, U.K.: Chapman \& Hall.

Baranoski, M. T. (2013). Structure Contour Map on the Precambrian Unconformity Surface in Ohio and Related Basement Features (Version 2.0). Columbus, Ohio Department of Natural Resources, Division of Geological Survey Map PG-23, Scale. Columbus: Ohio Department of Natural Resources.

Ben Wu, X., \& Smeins, F. E. (2000). Multiple-scale habitat modeling approach for rare plant conservation. Landscape and Urban Planning, 51(1), 11-28. https://doi.org/10.1016/S01692046(00)00095-5

Bettis, E. A., Benn, D. W., \& Hajic, E. R. (2008). Landscape Evolution, Alluvial Architecture, Environmental History, and the Archaeological Record of the Upper Mississippi River Valley. Geomorphology, 101(1), 362-377.

Biesele, M. (1993). Women Like Meat: The Folklore and Foraging Ideology of the Kalahari Ju/'hoan. Bloomington: Indiana University Press.

Binford, L. R. (2001). Constructing Frames of Reference: An Analytical Method for Archaeological Theory Building Using Hunter-Gatherer and Environmental Data Sets. Berkeley: University of California Press.

Blackmar, J. (2001). Regional variability in Clovis, Folsom, and Cody land use. Plains Anthropologist, 46(175), 65-94.

Blockland, J. D. (2013). The Surficial Geology of Fulton County, Ohio: Insight into the Late Pleistocene-Early Holocene Glaciated Landscape of the Huron-Erie Lake Plain, Fulton County, Ohio, USA. University of Toledo, Toledo.

Blott, S. J., \& Pye, K. (2001). GRADISTAT: A Grain Size Distribution and Statistics Package for the Analysis of Unconsolidated Sediments. Earth Surface Processes and Landforms, 26(11), 1237-1248.

Boisvert, R. A. (1984). Kentucky Salt Licks: A Preservation Planning Perspective. Lexington.

Boisvert, R. A. (2012). The Paleoindian Period in New Hampshire. In C. Chapdelaine (Ed.), Late Pleistocene Archaeology \& Ecology in the Far Northeast (pp. 77-94). College Station: Texas A\&M University Press.

Bond, G., Showers, W., Cheseby, M., Lotti, R., Almasi, P., DeMenocal, P., ... Bonani, G. (1997). A Pervasive Millennial-Scale Cycle in North Atlantic Holocene and Glacial Climates. Science, 278(5341), 1257-1266. https://doi.org/10.1126/science.278.5341.1257

Booth, R. K., Jackson, S. T., Forman, S. L., Kutzbach, J. E., Bettis, E. A., Kreig, J., \& Wright, D. K. (2005). A Severe Centennial-scale Drought in Midcontinental North America 4200 Years Ago and Apparent Global Linkages. The Holocene, 15(3), 321-328. https://doi.org/10.1191/0959683605hl825ft

Booth, R. K., Jackson, S. T., \& Gray, C. E. D. (2004). Paleoecology and High-resolution Paleohydrology of a Kettle Peatland in Upper Michigan. Quaternary Research, 61(1), 1-13. 
https://doi.org/10.1016/j.yqres.2003.07.013

Botkin, D. B., Jordan, P. A., Dominski, A. S., Lowendorf, H. S., \& Hutchinson, G. E. (1973). Sodium Dynamics in a Northern Ecosystem. Proceedings of the National Academy of Sciences of the United States of America, 70(10), 2745-2748.

Boulanger, M. T., Buchanan, B., O’Brien, M. J., Redmond, B. G., Glascock, M. D., \& Eren, M. I. (2015). Neutron Activation Analysis of 12,900-year-old Stone Artifacts Confirms 450 $510 \mathrm{~km}$ Clovis Tool-stone Acquisition at Paleo Crossing (33ME274), Northeast Ohio, USA. Journal of Archaeological Science, 53, 550-558.

Boulanger, M. T., \& Lyman, R. L. (2014). Northeastern North American Pleistocene Megafauna Chronologically Overlapped Minimally with Paleoindians. Quaternary Science Reviews, $85,35-46$.

Bowler, J. M. (1973). Clay Dunes: Their Occurrence, Formation and Environmental Significance. Earth-Science Reviews, 9, 315-338.

Bownocker, J. A. (1906). Salt Deposits and the Salt Industry in Ohio. Springfield: Springfield Publishing Company.

Brockman, C. S. (2006). Physiographic Regions of Ohio. Columbus: Ohio Division of Geological Survey, Map with table, 1:2,500,000, approximate scale.

Broster, J. B., Norton, M. R., Miller, D. S., Tune, J. W., \& Baker, J. D. (2013). Tennessee's Paleoindian Record: The Cumberland and Lower Tennessee River Watersheds. In J. A. M. Gingerich (Ed.), In the Eastern Fluted Point Tradition (pp. 299-314). Salt Lake City: The University of Utah Press.

Brown, I. W. (1999). Salt Manufacture and Trade from the Perspective of Avery Island, Louisana. Midcontinental Journal of Archaeology, 24(2), 113-151. Retrieved from http://www.jstor.org/stable/20708115

Brown, I. W. (2010). The Archaeology of Salt Springs in the Eastern Woodlands of the United States. In S. Li \& L. von Falkenhausen (Eds.), Salt Archaeology in China: Global Comparative Perspectives (Vol. 2) (pp. 375-409). Beijing: Science Press.

Bryan, A. L. (1977). Developmental Stages and Technological Traditions. Annals of the New York Academy of Sciences, 288(1), 355-368. https://doi.org/10.1111/j.17496632.1977.tb33628.x

Buchanan, B. (2003). The Effects of Sample Bias on Paleoindian Fluted Point Recovery in the United States. North American Archaeologist, 24(4), 311-338.

Buchanan, B., \& Collard, M. (2010). A Geometric Morphometrics-based Assessment of Blade Shape Differences among Paleoindian Projectile Point Types from Western North America. Journal of Archaeological Science, 37(2), 350-359. https://doi.org/10.1016/j.jas.2009.09.047

Buchanan, B., Hamilton, M. J., Kilby, J. D., \& Gingerich, J. A. M. (2016). Lithic Networks Reveal Early Regionalization in Late Pleistocene North America. Journal of Archaeological Science, 65, 114-121. https://doi.org/10.1016/j.jas.2015.11.003

Buchanan, B., O’Brien, M. J., \& Collard, M. (2014). Continent-wide or Region-specific? A Geometric Morphometrics-based Assessment of Variation in Clovis Point Shape. Archaeological and Anthropological Sciences, 6(2), 145-162.

Campbell, M. C., Fisher, T. G., \& Goble, R. J. (2011). Terrestrial Sensitivity to Abrupt Cooling Recorded by Aeolian Activity in Northwest Ohio, USA. Quaternary Research, 75(3), 411416. https://doi.org/10.1016/j.yqres.2011.01.009

Cannon, M. D., \& Meltzer, D. J. (2004). Early Paleoindian Foraging: Examining the Faunal 
Evidence for Large Mammal Specialization and Regional Variability in Prey Choice. Quaternary Science Reviews, 23(18-19), 1955-1987.

https://doi.org/http://dx.doi.org/10.1016/j.quascirev.2004.03.011

Cannon, M. D., \& Meltzer, D. J. (2008). Explaining Variability in Early Paleoindian Foraging. Quaternary International, 191(1), 5-17.

Carlson, E. H. (1991). Minerals of Ohio (Vol. Bulletin 6). Columbus: State of Ohio, Department of Natural Resources, Division of Geological Survey.

Carr, K. W., Adovasio, J. M., \& Vento, F. J. (2013). A Report on the 2008 Field Investigations at the Shoop Site (36DA20). In J. A. M. Gingerich (Ed.), In the Eastern Fluted Point Tradition (pp. 75-103). Salt Lake City: The University of Utah Press.

Chaplin, J. R., \& Mason, C. E. (1967). Geologic Map of the Garrison Quadrangle, KentuckyOhio, and Part of the Pond Run Quadrangle, Lewis County, Kentucky. Reston.

Chapman, F. R., \& Otto, M. P. (1976). An Archaeological Reconnaissance Survey of the Sandy Springs Area, Adams County, Ohio. Unpublished Report on File, Department of Archaeology, Ohio Historical Society.Columbus. Columbus: Ohio Historical Society.

Chappell, G. A. (1988). Identification and Correlation of the Fluvial Terraces in the Ohio River Valley Near Proctorville, Ohio and Cox Landing, West Virginia. Marshall University, Department of Geology, Huntington.

Charlton, R. (2008). Fundamentals of Fluvial Geomorphology. London: Routledge Publishing.

Childress, V. (2001). Gazetteer of Ohio Streams. Columbus: Ohio Department of Natural Resources, Division of Water.

Cochran, D. R., Richey, K. D., \& Maust, L. A. (1990). Early Paleoindian Economies in the Glaciated Regions of Indiana. Research in Economic Anthropology Supplement, 5, 143159.

Cohen, T. J., Nanson, G. C., Larsen, J. R., Jones, B. G., Price, D. M., Coleman, M., \& Pietsch, T. J. (2010). Late Quaternary Aeolian and Fluvial Interactions on the Cooper Creek Fan and the Association between Linear and Source-bordering Dunes, Strzelecki Desert, Australia. Quaternary Science Reviews, 29(3-4), 455-471. https://doi.org/10.1016/j.quascirev.2009.09.024

Collins, L., \& Collins, R. H. (1877). History of Kentucky. Louisville: Richard H. Collins.

Coogan, A. H. (1996). Ohio's Surface Rocks and Sediments (Ohio Divis). Fossils of Ohio: Ohio Division of Geological Survey Bulletin (Vol. 70). Columbus: Ohio Department of Natural Resources.

Cooke, R. U., Warren, A., \& Goudie, A. S. (1993). Desert Geomorphology. London: University College London Press.

Counts, R. C., Murari, M. K., Owen, L. A., Mahan, S. A., \& Greenan, M. (2015). Late Quaternary Chronostratigraphic Framework of Terraces and Alluvium along the Lower Ohio River, Southwestern Indiana and Western Kentucky, USA. Quaternary Science Reviews, 110, 72-91. https://doi.org/10.1016/j.quascirev.2014.11.011

Cramer, Z. (1814). The Navigator. Pittsburgh: Cramer, Spear and Eichbaum.

Cremeens, D. L., \& Mokma, D. L. (1986). Argillic Horizon Expression and Classification in the Soils of Two Michigan Hydrosequences. Soil Science Society of America Journal, 50, 1002-1007. https://doi.org/10.2136/sssaj1986.03615995005000040034x

Cunningham, R. M. (1973). Paleo-hunters along the Ohio River. Archaeology of Eastern North America, 1(1), 118-126.

Daniel Jr., I. R., Moore, C. R., \& Caynor, E. C. (2013). Sifting the Sands of Time: 
Geoarchaeology, Culture Chronology, and Climate Change at Squires Ridge, Northeastern North Carolina. Southeastern Archaeology, 32(2), 253-270. Retrieved from

http://search.ebscohost.com/login.aspx?direct=true \&db=f5h\&AN=94058474\&site=ehostlive

Dean, W. E. (1997). Rates, Timing, and Cyclicity of Holocene Eolian Activity in North-central United States: Evidence from Varved Lake Sediments. Geology, 25(4), 331-334. https://doi.org/10.1130/0091-7613(1997)025<0331:RTACOH >2.3.CO;2

Dean, W. E., Forester, R. M., \& Bradbury, J. P. (2002). Early Holocene Change in Atmospheric Circulation in the Northern Great Plains: An Upstream View of the 8.2 ka Cold Event. Quaternary Science Reviews, 21(16-17), 1763-1775. https://doi.org/10.1016/S02773791(02)00002-1

Death, R.G., Fuller, I.C., \& Macklin, M.G. (2015). Resetting the River Template: The Potential for Climate-related Extreme Floods to Transform River Geomorphology and Ecology. Freshwater Biology, 60(12), 2477-2496.

Delcourt, P. A., Delcourt, H. R., \& Davidson, J. L. (1983). Mapping and Calibration of Modern Pollen-vegetation Relationships in the Southeastern United States. Review of Palaeobotany and Palynology, 39(1), 1-45.

Denny, M., \& Benedetti-Cecchi, L. (2012). Scaling Up in Ecology: Mechanistic Approaches. Annual Review of Ecology, Evolution, and Systematics, 43(1), 1-22. https://doi.org/10.1146/annurev-ecolsys-102710-145103

Dethier, V. G. (1977). The Taste of Salt. American Scientist, 65(6), 744-751.

Dincauze, D. F. (1993). Fluted Points in the Eastern Forests. In O. Soffer \& N. D. Praslov (Eds.), From Kostenki to Clovis (pp. 279-292). New York: Springer.

Dorwin, J. T. (1966). Fluted Points and Late Pleistocene Geochronology in Indiana. Indiana Historical Society, Prehistory Research Series, 4, 145-188.

Drennen, B. C. (1974). Adams County Paleo-Indian District Nomination Form. Columbus: Ohio Historical Society.

Duke, D., \& King, J. (2014). A GIS model for predicting wetland habitat in the Great Basin at the Pleistocene-Holocene transition and implications for Paleoindian archaeology. Journal of Archaeological Science, 49(1), 276-291. https://doi.org/10.1016/j.jas.2014.05.012

Duller, G. A. T., Bøtter-Jensen, L., \& Murray, A. S. (2003). Combining infrared- and green-laser stimulation sources in single-grain luminescence measurements of feldspar and quartz. In Radiation Measurements (Vol. 37, pp. 543-550). https://doi.org/10.1016/S13504487(03)00050-7

Ellicott, A. (1803). The Journal of Andrew Ellicott. Philadelphia: Ayer Publishing.

Ellis, C. J. (2011). Measuring Paleoindian Range Mobility and Land-use in the Great Lakes/Northeast. Journal of Anthropological Archaeology, 30(3), 385-401. https://doi.org/http://dx.doi.org/10.1016/j.jaa.2011.05.001

Ellison, C. R. W. ., Chapman, M. R., \& Hall, I. R. (2006). Surface and Deep Ocean Interactions During the Cold Climate Event 8200 Years Ago. Science, 312(5782), 1929-1932. https://doi.org/10.1126/science.1127213

Eren, M. I., Buchanan, B., \& O’Brien, M. J. (2015). Social Learning and Technological Evolution during the Clovis Colonization of the New World. Journal of Human Evolution, 80, 159-170. https://doi.org/http://dx.doi.org/10.1016/j.jhevol.2015.01.002

Eren, M. I., Chao, A., Chiu, C.-H., Colwell, R. K., Buchanan, B., Boulanger, M. T., ... O’Brien, M. J. (2016). Statistical Analysis of Paradigmatic Class Richness Supports greater 
Paleoindian Projectile-point Diversity in the Southeast. American Antiquity, 81(1), 174192. https://doi.org/10.7183/0002-7316.81.1.174

Eren, M. I., \& Desjardine, A. (2015). Flaked Stone Tools of Pleistocene Colonizers: Overshot Flaking at the Red Wing Site, Ontario. In T. A. Jennings \& A. M. Smallwood (Eds.), Clovis: On the Edge of a New Understanding (pp. 109-120). College Station: Texas A\&M Press.

Eren, M. I., \& Redmond, B. G. (2011). Clovis Blades at Paleo Crossing (33ME274), Ohio. Midcontinental Journal of Archaeology, 36(2), 173-194.

Eren, M. I., Vanderlaan, S., \& Holland, J. D. (2011). Overshot Flaking at the Arc Site, Genesee County, New York: Examining the Clovis-Gainey Connection. The Open Anthropology Journal, 4, 40-52.

Evans, N. W., \& Stivers, E. B. (1900). A History of Adams County, Ohio. West Union: Emmons B. Stivers.

Fain, J., Soumana, S., Montret, M., Miallier, D., Pilleyre, T., \& Sanzelle, S. (1999).

Luminescence and ESR dating Beta-dose attenuation for various grain shapes calculated by a Monte-Carlo method. Quaternary Science Reviews. https://doi.org/10.1016/S02773791(98)00056-0

Fedoroff, N. (1974). Classification of Accumulations of Translocated Particles. In G. K. Rutherford (Ed.), Soil Microscopy (pp. 695-700, 711, 712). Kingston: Limestone Press.

Fenneman, N. M., \& Johnson, D. W. (1946). Physiographic Divisions of the Conterminous U.S. Washington, D.C.: US Geological Survey.

Field, A. (2009). Discovering Statistics using SPSS. Los Angeles: Sage publications.

FitzPatrick, E. A. (1984). The Micromorphology of Soils. London: Chapman and Hall.

Folk, R. L. (1974). Petrology of Sedimentary Rocks. Austin: Hemphill Publishing Company.

Folk, R. L., \& Ward, W. C. (1957). Brazos River Bar: A Study in the Significance of Grain Size Parameters. Journal of Sedimentary Research, 27(1), 3-26.

Forman, S. L. (2015). Episodic Eolian Sand Deposition in the Past 4000 Years in Cape Cod National Seashore, Massachusetts, USA in Response to Possible Hurricane/storm and Anthropogenic Disturbances. Frontiers in Earth Science, 3, 1-17. https://doi.org/https://doi.org/10.3389/feart.2015.00003

Forman, S. L., Marin, L., Pierson, J., Gómez, J., Miller, G. H., \& Webb, R. S. (2005). Aeolian Sand Depositional Records from Western Nebraska: Landscape Response to Droughts in the Past 1500 Years. The Holocene, 15(7), 973-981.

Forman, S. L., Oglesby, R., \& Webb, R. S. (2001). Temporal and Spatial Patterns of Holocene Dune Activity on the Great Plains of North America: Megadroughts and Climate Links. Global and Planetary Change, 29(1-2), 1-29. https://doi.org/http://dx.doi.org.www.libproxy.wvu.edu/10.1016/S0921-8181(00)00092-8

Forsyth, J. L. (1970). A Geologist Looks at the Natural Vegetation Map of Ohio. The Ohio Journal of Science, 70(3), 180-191.

Fowke, G. (1925). The Genesis of the Ohio River. Proceedings of the Indiana Academy of Science, 34, 81-102.

Fraser, D., \& Reardon, E. (1980). Attraction of Wild Ungulates to Mineral-Rich Springs in Central Canada. Holarctic Ecology, 3(1), 36-39.

Fraser, G. S. G. S., \& Fishbaugh, D. A. D. A. (1986). Alluviation of the Ohio River Valley Near Evansville, Indiana and its Effect on the Distribution of Sand and Gravel in the Area (Vol. Geological). Bloomington: Indiana Department of Natural Resources. 
Fredlund, G. G. (1989). Holocene Vegetational History of the Gallipolis Locks and Dam Project Area, Mason County, West Virginia (Vol. Contract P). Lexington: Cultural Resource Analysts, Inc.

Freeman, A. K. L., Smith, E. E., \& Tankersley, K. B. (1996). A Stone's Throw from Kimmswick: Clovis Period Research in Kentucky. In D. G. Anderson \& K. E. Sassaman (Eds.), The Paleoindian and Early Archaic Southeast (pp. 385-403). Tuscaloosa: The University of Alabama Press.

Friberg, J. F. (1970). Mineralogy and Provenance of the Recent Alluvial Sands of the Ohio River Drainage Basin (Ph.D.). Bloomington: Department of Geology, Indiana University.

Friedman, G. M. (1961). Distinction between Dune, Beach, and River Sands from their Textural Characteristics. Journal of Sedimentary Research, 31(4), 514-529.

Friedman, G. M. (1979). Address of the Retiring President of the International Association of Sedimentologists: Differences in size disributions of populations of Particles among Sands of Various Origins. Sedimentology, 26, 3-32.

Fritz, S. C., Metcalfe, S. E., \& Dean, W. (2001). Holocene Climate Patterns in the Americas Inferred from Paleolimnological Records. In Interhemispheric Climate Linkages (pp. 241263). https://doi.org/10.1016/B978-012472670-3/50018-5

Frokling, T. A., \& Szabo, J. P. (1998). Quaternary Geology along the Eastern Margin of the Scioto Lobe in Central Ohio. Columbus: Ohio Division of Geological Survey Guidebook No. 16.

Fryirs, K. A., \& Brierley, G. J. (2013). Geomorphic Analysis of River Systems: An Approach to Reading the Landscape. Oxford: John Wiley \& Sons.

Fullerton, D. S. (1986). Stratigraphy and Correlation of Glacial Deposits from Indiana to New York and New Jersey. Quaternary Science Reviews, 5, 23-37.

Galbraith, R. F., \& Green, P. F. (1990). Estimating the component ages in a finite mixture. International Journal of Radiation Applications and Instrumentation. Part D. Nuclear Tracks and Radiation Measurements, 17(3), 197-206.

Galbraith, R. F., \& Roberts, R. G. (2012). Statistical aspects of equivalent dose and error calculation and display in OSL dating: An overview and some recommendations. Quaternary Geochronology. https://doi.org/10.1016/j.quageo.2012.04.020

Galbraith, R. F., Roberts, R. G., Laslett, G. M., Yoshida, H., \& Olley, J. M. (1999). Optical Dating of Single and Multiple Grains of Quartz from Jimmum Rock Shelter, Northern Austrailia: Part I, Experimental Design and Statistical Models. Archaeometry, 41(2), 339364. https://doi.org/doi: 10.1111/j.1475- 4754.1999.tb00987.x

Gardner, W. M. (1974). The Flint Run Complex: Pattern and Process during the Paleoindian to Early Archaic. In W. M. Gardner (Ed.), The Flint Run Complex: A Preliminary Report 1971-73 Seasons (pp. 5-47). Washington, D.C.: Occasional Publication, No. 1, Archeology Laboratory, Department of Anthropology, The Catholic University of America.

Gardner, W. M. (1977). Flint Run Paleoindian Complex and Its Implications for Eastern North American Prehistory. Annals of the New York Academy of Sciences, 288(1), 257-263. https://doi.org/10.1111/j.1749-6632.1977.tb33621.x

Gilmore, M. R. (1919). Uses of Plants by the Indians of the Missouri River Region. Washington, D.C.: 33rd Annual Report of the Bureau of American Ethnology.

Gingerich, J. A. M. (2011). Down to Seeds and Stones: A New Look at the Subsistence Remains From Shawnee-Minisink. American Antiquity, 76(1), 127-144. https://doi.org/10.7183/0002-7316.76.1.127 
Gingerich, J. A. M. (2013a). Fifty Years of Discovery at Plenge: Rethinking the Importance of New Jersey's Largest Paleoindian Site. In J. A. M. Gingerich (Ed.), In the Eastern Fluted Point Tradition (pp. 121-147). Salt Lake City: The University of Utah Press.

Gingerich, J. A. M. (2013b). In the Eastern Fluted Point Tradition. (J. A. M. Gingerich, Ed.), In the Eastern Fluted Point Tradition. Salt Lake City: The University of Utah Press.

Gingerich, J. A. M. (2013c). Revisiting Shawnee-Minisink. In J. A. M. Gingerich (Ed.), In the Eastern Fluted Point Tradition (pp. 218-258). Salt Lake City: The University of Utah Press.

Gingerich, J. A. M. (2018). Discussion of the Eastern Paleoindian Record. In J. A. M. Gingerich (Ed.), In the Eastern Fluted Point Tradition, Volume II (pp. 409-430). Salt Lake City: The University of Utah Press.

Glasser, S., Gauthier-Warinner, J., Keely, J., Gurrieri, J., Tucci, P., Summers, P., ... McCormack, K. (2007). Technical Guide to Managing Ground Water Resources. United States Department of Agriculture: Washington, DC, USA. Washington, D.C.: United States Department of Agriculture.

Glover, K. C., Lowell, T. V, Wiles, G. C., Pair, D., Applegate, P., \& Hajdas, I. (2011). Deglaciation, Basin Formation and Post-glacial Climate Change from a Regional Network of Sediment Core Sites in Ohio and Eastern Indiana. Quaternary Research, 76, 401-410. https://doi.org/10.1016/j.yqres.2011.06.004

Goldthwait, R. P. (1958). Wisconsin Age Forests in Western Ohio. I, Age and Glacial Events. The Ohio Journal of Science, 58(4), 209-219. Retrieved from http://hdl.handle.net/1811/4546

Golledge, R. G. (2003). Human Wayfinding and Cognitive Maps. In M. Rockman \& J. Steele (Eds.), Colonization of Unfamiliar Landscapes: The Archaeology of Adaptation (pp. 2543). London: Routledge.

Goudie, A. S., \& Thomas, D. S. G. (1986). Lunette Dunes in Southern Africa. Journal of Arid Environments, 10, 1-12.

Gramly, R. M., \& Funk, R. E. (1990). What is Known and Not Known about the Human Occupation of the Northeastern United States until 10,000 BP. Archaeology of Eastern North America, 18, 5-31.

Gray, H. H. (1984). Archaeological Sedimentology of Overbank Silt Deposits on the Floodplain of the Ohio River near Louisville, Kentucky. Journal of Archaeological Science, 11, 421432.

Guisan, A., Weiss, S. B., \& Weiss, A. D. (1999). GLM versus CCA Spatial Modeling of Plant Species Distribution. Plant Ecology, 143(1), 107-122. Retrieved from http://www.jstor.org/stable/20050798

Gupta, R. K., Singh, R. R., \& Abrol, I. P. (1989). Influence of Simultaneous Changes in Sodicity and $\mathrm{pH}$ on the Hydraulic Conductivity of an Alkali Soil Under Rice Culture. Soil Science, $147(1), 28-33$.

Halligan, J. J. (2013). Reconstructing the Pleistocene Environment of the Greater Southeast. In J. A. M. Gingerich (Ed.), In the Eastern Fluted Point Tradition (pp. 58-74). Salt Lake City: The University of Utah Press.

Hansen, E., Arbogast, A., Packman, S., \& Hansen, B. (2002). Post-Nipissing Origin of a Backdune Complex Along the Southeastern Shore of Lake Michigan. Physical Geography, 23(3), 233-244. https://doi.org/10.2747/0272-3646.23.3.233

Hansen, M. C. (1983). Ohio's Salt Industry. Ohio Geology Newsletter, Division of Geological 
Survey, 1983(Fall), 1-6.

Hansen, M. C. (1995). The Scioto Saline - Ohio's Early Salt Industry. Division of Geological Survey GeoFacts (Vol. GeoFacts N). Columbus: Ohio Department of Natural Resources, Division of Geological Survey.

Hansen, M. C. (1995). The Teays River: Ohio Department of Natural Resources. Division of Geological Survey, GeoFacts (Vol. GeoFacts N). Columbus: Ohio Department of Natural Resources, Division of Geological Survey.

Hanson, P. R., Arbogast, A. F., Johnson, W. C., Joeckel, R. M., \& Young, A. R. (2010). Megadroughts and Late Holocene Dune Activation at the Eastern Margin of the Great Plains, North-central Kansas, USA. Aeolian Research, 1(3-4), 101-110. https://doi.org/http://dx.doi.org/10.1016/j.aeolia.2009.10.002

Harris, T. M. (2006). Scale as Artifact: GIS, Ecological Fallacy, and Archaeological Analysis. In G. Lock \& B. L. Molyneaux (Eds.), Confronting Scale in Archaeology (pp. 39-54). New York: Springer.

Harvey, D. (1968). Processes, patterns and scale problems in geographical research. Transactions of the Institute of British Geographers, 45, 71-78.

Haynes, C. V. (1964). Fluted Projectile Points: Their Age and Dispersion: Stratigraphically controlled radiocarbon dating provides new evidence on peopling of the New World. Science, 145(3639), 1408-1413. https://doi.org/10.1126/science.145.3639.1408

Hellgren, E. C., \& Pitts, W. J. (1997). Sodium Economy in White-tailed Deer (Odocoileus virginianus). Physiological and Biochemical Zoology, 70(5), 547-555.

Hem, J. D. (1985). Study and Interpretation of the Chemical Characteristics of Natural Water (Third). Alexandria: U.S. Geological Survey Water-Supply Paper 2254.

Hemmings, C. A. (2004). The Organic Clovis: A Single Continent-wide Cultural Adaptation. University of Florida, Gainesville U6 - ctx_ver=Z39.882004\&ctx_enc $=$ info\%3Aofi $\% 2$ Fenc\%3AUTF-

8\&rfr_id=info:sid/summon.serialssolutions.com\&rft_val_fmt=info:ofi/fmt:kev:mtx:disserta tion\&rft.genre $=$ dissertation\&rft.title $=$ The + organic $+\overline{\text { Clovis }} \% 3 \mathrm{~A}+\mathrm{A}+$ single + continent wide+cultural+adapta. Retrieved from http://wvu.summon.serialssolutions.com/2.0.0/link/0/eLvHCXMwnV1Ra8IwED7cnmSDb UzZ3IT8gW5pU1Lji4ib7AfsXa5JBKFUnXX_d01LYhuL3tMAuFow3eXuy_3AajkRUYnmEBuzCqT54bCh3QUo0WDGaegyHvGWMu pHLXthrYrRPO3W5CskdutLSfNXmgcmycqMlmG7GKFFdbG0kNguQ40SbUbk8v72eoW7uS-Q20hNmGWx1

Hildreth, S. P. (1838). Salt Springs. Ohio Division of Geological Survey, 54-62.

Hillel, D. (2013). Fundamentals of Soil Physics. New York: Academic press.

Hollenbach, K. D. (2009). Foraging in the Tennessee River Valley: 12,500 to 8,000 years ago. Tuscaloosa: University of Alabama Press. Retrieved from https://books.google.com/books?hl=en\&lr=\&id=MBxt3IzNq5QC\&oi=fnd\&pg=PR7\&dq=F oraging + in + the + Tennessee + River + Valley $,+12,500+$ to $+8,000+$ years + ago\&ots $=0 \mathrm{M} \_$vuspulF $\&$ sig=fw1 PLCsdKlgKoubFEBxKxBqDqAM

Holliday, V. T., \& Miller, D. S. (2014). The Clovis Landscape. In K. E. Graf, C. V Ketron, \& M. R. Waters (Eds.), Paleoamerican Odyssey (pp. 221-245). College Station: Texas A\&M University Press.

Hopkins, H. T. (1966). The Fresh-saline Water Interface in Kentucky. (10th ed.). Lexington: Kentucky Geological Survey. 
Hu, F. S., Slawinski, D., Wright, H. E., Ito, E., Johnson, R. G., Kelts, K. R., .. Boedigheimer, A. (1999). Abrupt Changes in North American Climate during Early Holocene Times. Nature, 400(6743), 437-440. https://doi.org/Doi 10.1038/22728

Hubbard, G. D. (1954). Terrace Interpretation in Southeastern Ohio. The Ohio Journal of Science, 54(6), 365-377.

Hull, D. N. (1990). Generalized Column of Bedrock Units in Ohio. Ohio, Ohio Department of Natural Resources, Geological Survey. Columbus: Ohio Department of Natural Resources, Division of Geological Survey.

Hunter, R. E. (1977). Basic types of stratification in small eolian dunes. Sedimentology, 24(3), 361-387. https://doi.org/10.1111/j.1365-3091.1977.tb00128.x

Imlay, G. (1793). A Topographical Description of the Western Territory of North America. London: William Jones.

Ivester, A. H., \& Leigh, D. S. (2003). Riverine Dunes on the Coastal Plain of Georgia, USA. Geomorphology, 51(4), 289-311. https://doi.org/http://dx.doi.org/10.1016/S0169$555 \mathrm{X}(02) 00240-4$

Jackson, L. J. (1990). Interior Paleoindian Settlement Strategies: A First Approximation for the Lower Great Lakes. Early Paleoindian Economies of Eastern North America, 5, 95-142.

Jackson, L. J., Ellis, C., Morgan, A. V., \& McAndrews, J. H. (2000). Glacial Lake Levels and Eastern Great Lakes Palaeo-Indians. Geoarchaeology - An International Journal, 15(5), 415-440. https://doi.org/10.1002/(SICI)1520-6548(200006)15:5<415::AIDGEA2>3.0.CO;2-2

Jacobson, R. B., Elston, D. P., \& Heaton, J. W. (1988). Stratigraphy and Magnetic Polarity of the High Terrace Remnants in the Upper Ohio and Monongahela Rivers in West Virginia, Pennsylvania, and Ohio. Quaternary Research, 29(3), 216-232.

Jakle, J. A. (1969). Salt on the Ohio Valley Frontier, 1770-1820. Annals of Teh Association of American Geographers, 59(4), 687-709.

Jenness, J. (2006). Topographic Position Index (tpi_jen.avx) extension for ArcView 3.x, v. 1.2. Jodry, M. A. (2005). Envisioning Water Transport Technology in Late-Pleistocene America. In R. Bonnichsen, B. T. Lepper, D. Stanford, \& M. R. Waters (Eds.), Paleoamerican Origins: Beyond Clovis (pp. 133-160). College Station: Texas A\&M University Press.

Johnson, E., Politis, G., Gutierrrez, M., Martinez, G., \& Miotti, L. (2006). Grassland Archaeology in the Americas: From the U.S. Southern Plains to the Argentinean Pampas. In J. E. Morrow \& C. Gnecco (Eds.), Paleoindian Archaeology: A Hemispheric Perspective (pp. 44-68). Gainseville: University Press of Florida.

Jones, R. R. (1916). The Ohio River: Charts, Drawings, and Description of Features Affecting Navigation. Washington, D.C.: Government Printing Office.

Jordan, P. (2008). Hunters and Gatherers. In R. A. Bentley, H. D. G. Maschner, \& C. Chippindale (Eds.), Handbook of Archaeological Theories (pp. 447-466). New York: Altamira Press.

Justice, N. D. (1987). Stone Age Spear and Arrow Points of the Midcontinental and Eastern United States: A Modern Survey and Reference. Bloomington: Indiana University Press.

Karathanasis, A. D., \& Macneal, B. R. (1994). Evaluation of Parent Material Uniformity Criteria in Loess-influenced Soils of West-central Kentucky. Geoderma, 64(1-2), 73-92. https://doi.org/10.1016/0016-7061(94)90090-6

Kasper-Zubillaga, J. J., \& Carranza-Edwards, A. (2005). Grain Size Discrimination between Sands of Desert and Coastal Dunes from Northwestern Mexico. Revista Mexicana de 
Ciencias Geologicas, 22(3), 383-390.

Kelly, R. L. (1995). The Foraging Spectrum. Washington, D.C.: Smithsonian Institution Press.

Kelly, R. L. (2003). Colonization of New Land by Hunter-Gatherers. In M. Rockman \& J. Steele (Eds.), Colonization of Unfamiliar Landscapes: The Archaeology of Adaptation (pp. 4458). London: Routledge.

Kelly, R. L., \& Todd, L. C. (1988). Coming into the Country: Early Paleoindian Hunting and Mobility. American Antiquity, 53(2), 231-244.

Kempton, J. P., \& Goldthwait, R. P. (1959). Glacial Outwash Terraces of the Hocking and Scioto River Valleys, Ohio. The Ohio Journal of Science, 59(3), 135-151.

Kidder, T. R., Adelsberger, K. A., Arco, L. J., \& Schilling, T. M. (2008). Basin-scale Reconstruction of the Geological Context of Human Settlement: An Example from the Lower Mississippi Valley, USA. Quaternary Science Reviews, 27(11), 1255-1270.

Kilibarda, Z., Argyilan, E., \& Blockland, J. (2008). Wind Deposition of Mud Aggregates and their Role in Development of Lamellae in the Fair Oaks Dunes, Indiana. Catena, 72(2), $235-247$. https://doi.org/http://dx.doi.org.www.libproxy.wvu.edu/10.1016/j.catena.2007.05.006

Kilibarda, Z., \& Blockland, J. (2011). Morphology and Origin of the Fair Oaks Dunes in NW Indiana, USA. Geomorphology, 125(2), 305-318. https://doi.org/10.1016/j.geomorph.2010.10.011

Kilibarda, Z., Venturelli, R., \& Goble, R. (2014). Late Holocene Dune Development and Shift in Dune-building Winds along Southern Lake Michigan. Special Paper of the Geological Society of America (Vol. 508). https://doi.org/10.1130/2014.2508(04)

Klemsdal, T. (2010). The Eolian Landforms and Sediment in the Valley of River Jømna, East of Elverum, South-east Norway. Norsk Geografisk Tidsskrift - Norwegian Journal of Geography, 64(2), 94-104. https://doi.org/10.1080/00291951.2010.481138

Klovan, J. E. (1966). The Use of Factor Analysis in Determining Depositional Environments from Grain Size Distributions. Journal of Sedimentary Petrology, 36, 115-125.

Kobashi, T., Severinghaus, J. P., Brook, E. J., Barnola, J. M., \& Grachev, A. M. (2007). Precise Timing and Characterization of Abrupt Climate Change 8200 Years Ago from Air Trapped in Polar Ice. Quaternary Science Reviews, 26(9-10), 1212-1222.

https://doi.org/10.1016/j.quascirev.2007.01.009

Kocurek, G. (1996). Desert Aeolian Systems. In H. G. Reading (Ed.), Sedimentary Environments: Processes, Facies and Stratigraphy (pp. 125-153). Oxford: Blackwell Science Ltd.

Kocurek, G., \& Lancaster, N. (1999). Aeolian System Sediment State: Theory and Mojave Desert Kelso Dune Field Example. Sedimentology, 46(3), 505-515. https://doi.org/10.1046/j.1365-3091.1999.00227.x

Koldehoff, B., \& Loebel, T. J. (2009). Clovis and Dalton: Unbounded and Bounded Systems in the Midcontinent of North America. In B. Adams \& B. S. Blades (Eds.), Lithic Materials and Paleolithic Societies (pp. 270-287). West Sussex: Blackwell Publishing.

Kraft, H. C. (1973). The Plenge Site: A Paleo-indian Occupation Site in New Jersey. Archaeology of Eastern North America, 1(1), 56-117.

Kreulen, D. A. (1985). Lick use by large herbivores: A review of benefits and banes of soil consumption. Mammal Rev., 15, 107-123.

Krieg, J. R., Bettis, E. A., \& Forman, S. L. (2004). Evidence for late Holocene eolian sand reactivation in the Green River lowland, northwestern Illinois; regional response to drought 
and potential implications. Abstracts with Programs - Geological Society of America, 36(5), 70 .

Kvamme, K. L. (2006). There and Back Again: Revisiting Archaeological Locational Modeling. In M. W. Mehrer \& K. L. Wescott (Eds.), GIS and Archaeological Site Location Modeling (pp. 3-38). Boca Raton: CRC Press.

Lam, N. S.-N. N., \& Quattrochi, D. A. (1992). On the issues of scale, resolution, and fractal analysis in the mapping sciences. Professional Geographer, 44(1), 88-98. https://doi.org/10.1111/j.0033-0124.1992.00088.x

Lancaster, N. (1981). Paleoenvironmental Implications of Fixed Dune Systems in Southern Africa. Paleogeography, Palaeoclimatology, Palaeoecology, 33, 327-346.

Lancaster, N. (2011). Desert Dune Processes and Dynamics. In D. S. G. Thomas (Ed.), Arid Zone Geomorphology: Process, Form and Change in Drylands (3rd ed., pp. 487-515). Oxford: Wiley-Blackwell.

Lane, L., \& Anderson, D. G. (2001). Paleoindian Occupations of the Southern Appalachians: A View from the Cumberland Plateau of Kentucky and Tennessee. In L. P. Sullivan Prezzano,Susan C., (Ed.), Archaeology of the Appalachian highlands (pp. 88-102). Knoxville: University of Tennessee Press.

Langford, R. P. (1989). Fluvial-aeolian Interactions: Part I, Modern Systems. Sedimentology, 36(6), 1023-1035. https://doi.org/10.1111/j.1365-3091.1989.tb01540.x

Legg, R. J., Regis, R. R., Lambert, J. M., Liesch, M., \& Travis, C. B. (2017). Geoarchaeological Modeling of Late Paleoindian Site Location in the Northwestern Great Lakes Region. PaleoAmerica, 3(2), 150-160.

Leigh, D. S. (1998). Evaluating Artifact Burial by Eolian Versus Bioturbation Processes, South Carolina Sandhills, USA. Geoarchaeology, 13(3), 309-330.

Leigh, D. S. (2006). Terminal Pleistocene Braided to Meandering Transition in Rivers of the Southeastern USA. Catena, 66(1), 155-160.

Leigh, D. S. (2008). Late Quaternary Climates and River Channels of the Atlantic Coastal Plain, Southeastern USA. The 39th Annual Binghamton Geomorphology Symposium: Fluvial Deposits and Environmental History: Geoarchaeology, Paleohydrology, and Adjustment to Environmental Change, 101(1-2), 90-108.

https://doi.org/http://dx.doi.org/10.1016/j.geomorph.2008.05.024

Leigh, D. S., Srivastava, P., \& Brook, G. A. (2004). Late Pleistocene Braided Rivers of the Atlantic Coastal Plain, USA. Quaternary Science Reviews, 23(1), 65-84.

Leopold, L. B., Wolman, M. G., \& Miller, J. P. (1964). Fluvial Processes in Geomorphhology. New York: Dover Publications, Inc.

Lepper, B. T. (1983). Fluted Point Distributional Patterns in the Eastern United States: A Contemporary Phenomenon. Midcontinental Journal of Archaeology, 8(2), 269-285.

Lepper, B. T. (1985). The Effects of Cultivation and Collecting on Ohio Fluted Point Finds: A Reply to Seeman and Prufer. Midcontinental Journal of Archaeology, 10(2), 241-250.

Lepper, B. T. (1986). Early Paleo-Indian Land Use Patterns in the Central Muskingum River Basin, Coshocton County, Ohio. Early Paleo-Indian Land Use Patterns in the Central Muskingum River Basin, Coshocton County, Ohio. The Ohio State University, Department of Anthropology, Columbus.

Lepper, B. T. (1988). Early Paleo-Indian Foragers of Midcontinental North America. North American Archaeologist, 9(1). https://doi.org/10.2190/UK8E-GYAX-FMKM-89CN

Lepper, B. T., \& Meltzer, D. J. (1991). Late Pleistocene Human Occupation of the Eastern 
United States. In R. Bonnichsen \& K. L. Turnmire (Eds.), Clovis: Origins and Adaptations (pp. 175-184). Corvallis: Center for the Study of the First Americans, Oregon State University.

Lewis, T. M. N. (1953). The Paleo-Indian Problem in Tennessee. Tennessee Archaeologist, 9, 38-40.

Li, Y.-X., Yu, Z., \& Kodama, K. P. (2007). Sensitive Moisture Response to Holocene Millennial-scale Climate Variations in the Mid-Atlantic Region, USA. The Holocene, 17(1), 3-8. https://doi.org/10.1177/0959683606069386

Liu, B., \& Coulthard, T. J. (2015). Mapping the Interactions between Rivers and Sand Dunes: Implications for Fluvial and Aeolian Geomorphology. Geomorphology, 231, 246-257. https://doi.org/http://dx.doi.org.www.libproxy.wvu.edu/10.1016/j.geomorph.2014.12.011

Liu, Y., Andersen, J. J., Williams, J. W., \& Jackson, S. T. (2013). Vegetation History in Central Kentucky and Tennessee (USA) during the Last Glacial and Deglacial Periods. Quaternary Research, 79(2), 189-198.

Lock, G. R., \& Harris, T. M. (2000). Introduction: Return to Ravello. In G. R. Lock (Ed.), Beyond the Map: Archaeology and Spatial Technologies (pp. xiii-xxv). Ravello: IOS Press.

Lock, G. R., \& Harris, T. M. (2006). Enhancing Predictive Archaeological Modeling: Integrating Location, Landscape, and Culture. In M. W. Mehrer \& K. L. Wescott (Eds.), GIS and Archaeological Site Location Modeling (pp. 36-55). Boca Raton: CRC Press.

Loebel, T. J. (2012). Pattern or Bias? A Critical Evaluation of Midwestern Fluted Point Distributions using Raster Based GIS. Journal of Archaeological Science, 39(5), 12051217.

Lombardo, U., \& Prümers, H. (2010). Pre-Columbian human occupation patterns in the eastern plains of the Llanos de Moxos, Bolivian Amazonia. Journal of Archaeological Science, 37(8), 1875-1885. https://doi.org/10.1016/j.jas.2010.02.011

Lothrop, J. C., \& Bradley, J. W. (2012). Paleoindian Occupations in the Hudson Valley, New York. In C. Chapdelaine (Ed.), Late Pleistocene Archaeology \& Ecology in the Far Northeast (pp. 9-47). College Station: Tempus Publishing. Retrieved from https://books.google.com/books?hl=en\&lr=\&id=DfV9YSOwFwEC\&oi=fnd\&pg=PA9\&dq $=$ Paleoindian+occupations + in + the + Hudson + Valley,+ New + York\&ots $=$ NFZonJDxF_\&sig $=$ 7xgfLXvXTVSe3W2hWSwDm77RPaI

Lothrop, J. C., \& Cremeens, D. L. (2010). 33Ms391: A Paleoindian Site in Southwestern Ohio. Current Research in the Pleistocene, 27, 120-122.

Lothrop, J. C., Lowery, D. L., Spiess, A. E., \& Ellis, C. J. (2016). Early Human Settlement of Northeastern North America. PaleoAmerica, 2(3), 192-251.

https://doi.org/10.1080/20555563.2016.1212178

Lucht, T. E., \& Brown, D. L. (1994). Soil Survey of Adams County, Ohio. Columbus: United States Department of Agriculture - Soil Conservation Service.

Lutz, B., Wiles, G., Lowell, T., \& Michaels, J. (2007). The 8.2-ka Abrupt Climate Change Event in Brown's Lake, Northeast Ohio. Quaternary Research, 67(2), 292-296. https://doi.org/10.1016/j.yqres.2006.08.007

Maggard, G. J., \& Stackelbeck, K. L. (2008). Paleoindian Period. In D. Pollack (Ed.), The Archaeology of Kentucky: An Update (Volume I) (pp. 73-192). Frankfort: The Kentucky Heritage Council.

Mandel, R. D. (1988). Geomorphology of the Ohio River Valley at the Gallipolis Locks and Dam, West Virginia. Lexington: Cultural Resource Analysts, Inc. 
Markewich, H. W., \& Markewich, W. (1994). An Overview of Pleistocene and Holocene Inland Dunes in Georgia and the Carolinas: Morphology, Distribution, Age, and Paleoclimate (Vol. U.S. Geolo). Washington: U.S. Government Printing Office.

Mason, J. A., Jacobs, P. M., Greene, R. S. B., \& Nettleton, W. D. (2003). Sedimentary Aggregates in the Peoria Loess of Nebraska, USA. Catena, 53(4), 377-397. https://doi.org/http://dx.doi.org/10.1016/S0341-8162(03)00073-0

Mason, J. A., Swinehart, J. B., Goble, R. J., \& Loope, D. B. (2004). Late-Holocene Dune Activity Linked to Hydrological Drought, Nebraska Sand Hills, USA. Holocene, 14(2), 209-217. https://doi.org/10.1191/0959683604hl677rp

Mason, J. A., Swinehart, J. B., Hanson, P. R., Loope, D. B., Goble, R. J., Miao, X., \& Schmeisser, R. L. (2011). Late Pleistocene Dune Activity in the Central Great Plains, USA. Quaternary Science Reviews, 30(27-28), 3858-3870. https://doi.org/http://dx.doi.org/10.1016/j.quascirev.2011.10.005

Mason, J. P., Swinehart, J. B., \& Loope, D. B. (1997). Holocene History of Lacustrine and Marsh Sediments in a Dune-blocked Drainage, Southwestern Nebraska Sand Hills, U.S.A. Journal of Paleolimnology, 17(1), 67-83. https://doi.org/10.1023/A:1007917110965

Mason, R. J. (1962). The Paleo-Indian Tradition in Eastern North America. Current Anthropology, 3(3), 227-278. https://doi.org/10.1086/200289

McAvoy, W. A., \& Harrison, J. W. (2012). Plant community classification and the flora of Native American shell-middens on the Delmarva Peninsula. Maryland Naturalist, 52, 1-34.

McCartney, D. M., Finney, M. A., \& Maynard, J. B. (2005). Sources of the Salt in the Big Bone Lick Springs, Northern Kentucky. Denver: Geological Society of America.

McCary, B. C. (1951). A Workshop Site of Early Man in Dinwiddie County, Virginia. American Antiquity, 17(1), 9-17.

McCord, B. K., \& Cochran, D. R. (2008). The Adena Complex: Identity and Context in EastCentral Indiana. In M. P. Otto \& B. G. Redmond (Eds.), Transitions: Archaic and Early Woodland Research in the Ohio Country (pp. 334-361). Athens: Ohio University Press.

McWeeney, L. J., \& McWeeney J., L. (2013). Paleoindian Environment and Subsistence Paradigm Case from New England to Virginia and Ohio. In J. A. M. Gingerich (Ed.), In the Eastern Fluted Point Tradition (pp. 38-57). Salt Lake City: The University of Utah Press.

Mejdahl, V., \& Christiansen, H. H. (1994). Procedures used for luminescence dating of sediments. Quaternary Science Reviews, 13(5-7), 403-406. https://doi.org/10.1016/02773791(94)90049-3

Meltzer, D. J. (1985). On Stone Procurement and Settlement Mobility in Eastern Fluted Point Groups. North American Archaeologist, 6(1), 1-24. https://doi.org/10.2190/T0JL-P9E8X45J-QBDX

Meltzer, D. J. (1988). Late Pleistocene Human Adaptations in Eastern North America. Journal of World Prehistory, 2(1), 1-52. https://doi.org/10.1007/BF00975121

Meltzer, D. J. (2003). Lessons in Landscape Learning. In M. Rockman \& J. Steele (Eds.), Colonization of Unfamiliar Landscapes: The Archaeology of Adaptation (pp. 222-241). London: Routledge Publishing.

Meltzer, D. J. (2009). First Peoples in a New World: Colonizing Ice Age America. Oakland: University of California Press.

Meltzer, D. J., \& Holliday, V. T. (2010). Would North American Paleoindians have Noticed Younger Dryas Age Climate Changes? Journal of World Prehistory, 23(1), 1-41.

Meltzer, D. J., \& Smith, B. D. (1986). Paleoindian and Early Archaic Subsistence Strategies in 
Eastern North America. In S. Neusius (Ed.), Foraging, Collecting and Harvesting: ArchaicPeriod Subsistence and Settlement in the Eastern Woodlands (pp. 2-31). Carbondale: Center for Archaeological investigations Occasional Paper.

Miao, X., Hanson, P. R., Wang, H., \& Young, A. R. (2010). Timing and Origin for Sand Dunes in the Green River Lowland of Illinois, Upper Mississippi River Valley, USA. Quaternary Science Reviews, 29(5), 763-773.

Miller, D. S., Gingerich, J. A. M. M., Miller, D. S., Gingerich, J. A. M. M., Miller, D. S., \& Gingerich, J. A. M. M. (2013). Paleoindian Chronology and the Eastern Fluted Point Tradition. In J. A. M. Gingerich (Ed.), In the Eastern Fluted Point Tradition (pp. 9-37). Salt Lake City: The University of Utah Press. Retrieved from https://scholar.google.com/scholar?hl=en\&as_sdt=0\%2C15\&q=Paleoindian + Chronology $+\mathrm{a}$ nd + the + Eastern+Fluted + Point + Tradition\&btnG $=$

Miller, G. L., Bebber, M. R., Rutkoski, A., Haythorn, R., Boulanger, M. T., Buchanan, B., ... Eren, M. I. (2018). Hunter-gatherer Gatherings: Stone-tool Microwear from the Welling Site (33-Co-2), Ohio, U.S.A. supports Clovis use of Outcrop-related Base Camps during the Pleistocene Peopling of the Americas. World Archaeology.

https://doi.org/10.1080/00438243.2018.1461128

Miller, J. J., Pawluk, S., \& Beke, G. J. (1993). Soil Salinization at a Side-hill Seep and Closed Basin in Southern Alberta. Canadian Journal of Soil Science, 73, 209-222.

Moerman, D. E. (1988). Native American Ethnobotany. Portland: Timber Press.

Moiola, R. J., \& Spencer, A. B. (1979). Differentiation of Eolian Deposits by Discriminant Analysis. In E. D. McKee (Ed.), A Study of Global Sand Seas (pp. 53-60). Washington, D.C.: United States Government Printing Office, Geological Survey Professional Paper 1052.

Moiola, R. J., Spencer, A. B., \& Weiser, D. (1974). Differentiation of Modern Sand Bodies by Linear Discriminant Analysis. Gulf Coast Association of Geological Societies Transactions, $24,321-326$.

Moore, C. R., \& Daniel Jr., I. R. (2011). Geoarchaeological Investigations of Stratified Sand Dune Ridges along the Tar River, North Carolina. In C. R. Ewen, T. R. Whyte, \& R. P. S. D. Davis Jr. (Eds.), The Archaeology of North Carolina: Three Archaeological Symposia (pp. 1-42). Raleigh: North Carolina Archaeological Council.

Morris, R. H., \& Pierce, K. L. (1967). Geologic Map of the Vanceburg Quadrangle, KentuckyOhio. Reston: U.S. Geological Survey.

Morrow, J. E. (2014). Early Paleoindian Mobility and Watercraft: An Assessment from the Mississippi River Valley. Midcontinental Journal of Archaeology, 39(2), 103-129.

Moseley, E. L. (1931). Some Plants that were probably brought to Northern Ohio from the West by Indians. Papers of the Michigan Academy of Sciences, Arts \& Letters, 13, 169-172.

Mullett, A. N. (2009). Paleoindian Mobility Ranges Predicted by the Distribution of Projectile Points Made of Upper Mercer and Flint Ridge Flint. Kent State University, Department of Anthropology, Kent.

Murray, A. S., \& Wintle, A. G. (2003). The Single Aliquot Regenerative Dose Protocol: Potential for Improvements in Reliability. Radiation Measurements, 37, 377-381. https://doi.org/doi: 10.1016/S1350-4487(03)00053-2

Nakoinz, O. (2010). Concepts of Central Place Research in Archaeology. In J. MÜLLER (Ed.), Landscapes and Human Development: The Contribution of European Archaeology (pp. 251-264). Bonn: Rudolf Habelt. 
National Climatic Data Center. (1996). Climatic Wind Data for the United States: 1930-1996. Retrieved July 4, 2017, from https://www.ncdc.noaa.gov

NOAA. (2017). National Weather Service. Retrieved January 6, 2017, from http://water.weather.gov

Noelle, H. J., \& Blackwell, W. H. (1972). The Cactaceae in Ohio. Castanea, 37(2), 119-124.

O'Brien, M. J. (1996). Paradigms of the Past: The Story of Missouri Archaeology. Columbia: University of Missouri Press.

O’Shea, J., Lemke, A. K., \& Reynolds, R. G. (2013). 'Nobody Knows the way of the Caribou'”: Rangifer hunting at $45^{\circ}$ North Latitude. Quaternary International, 297, 36-44. https://doi.org/10.1016/j.quaint.2013.01.010

ODNR. (2017). ODNR Division of Water Resources. Retrieved January 1, 2017, from http://water.ohiodnr.gov/search-file-well-logs

OGRIP. (2015). Ohio Geographically Referenced Information Program. Retrieved June 6, 2015, from http://ogrip.oit.ohio.gov/

Pavey, R. R., Goldthwait, R. P., Brockman, C. S., Hull, D. N., Swinford, E. Mac, \& Van Horn, R. G. (1999). Quaternary Geology of Ohio. Columbus: Ohio Division of Geological Survey.

Peel, M.C.; Finlayson, B.L.; McMahon, T. A. (2007). Updated World Map of the KöppenGeiger Climate Classification. Hydrology and Earth System Sciences, 11, 1633-1644. https://doi.org/https://doi.org/10.5194/hess-11-1633-2007, 2007

Pevny, C. D., Thulman, D. K., \& Faught, M. K. (2018). Ancestor-Descendant Relationships in the Early Holocene: Evidence for Clovis Paleoindian-Bolen Early Archaic Cultural Continuity in Florida. In J. A. M. Gingerich (Ed.), In the Eastern Fluted Point Tradition, Volume II (pp. 213-239). Salt Lake City: The University of Utah Press.

Phalen, W. C. (1919). Salt Resources of the United States (Vol. Bulletin 6). Washington, DC: United States Geological Survey. Retrieved from http://purl.fdlp.gov/GPO/gpo59827

Pitblado, B. L., Cannon, M. B., \& Fowler, B. (2011). Predictive Modeling of the Paleoindian Record in Southeastern Idaho and Northern Utah. Idaho Archaeologist, 34, 21-34.

Poppe, L. J., McMullen, K. Y., Williams, S. J., \& Paskevich, V. F. (2014). USGS East-coast Sediment Analysis: Procedures, Database, and GIS data. U.S. Geological Survey Open-File Report 2005-1001. Reston: U.S. Geological Survey.

Potter, P. E. (2007). Exploring the Geology of the Cincinnati/Northern Kentucky Region (Second Revised Edition) (Series XII, Vol. Special Pu). Lexington: Kentucky Geological Survey, University of Kentucky.

Powers, M. C. (1953). A New Roundness Scale for Sedimentary Particles. Journal of Sedimentary Research, 23, 117-119. https://doi.org/10.1306/D4269567-2B26-11D7$8648000102 \mathrm{C} 1865 \mathrm{D}$

Prasciunas, M. M. (2011). Mapping Clovis: Projectile Points, Behavior, and Bias. American Archaeology, 76, 107-126. https://doi.org/10.7183/0002-7316.76.1.107

Prescott, J. R., \& Hutton, J. T. (1994). Cosmic Ray Contributions to Dose Rates for Luminescence and ESR Dating: Large Depths and Long-term Time Variations. Radiation Measurements, 23, 497-500. https://doi.org/doi: 10.1016/1350- 4487(94)90086-8

Prufer, O. H. (1963). The McConnell Site: A Late Palaeo-Indian Workshop in Coshocton County, Ohio. Cleveland: Cleveland Museum of Natural History.

Prufer, O. H. (1975). The Scioto Valley Archaeological Survey. In O. H. Prufer \& D. H. McKenzie (Eds.), Studies in Ohio Archaeology (pp. 267-328). Kent: Kent State University Press. 
Prufer, O. H., \& Baby, R. S. (1963). The Paleo-indians of Ohio. Columbus: Ohio Historical Society.

Purkait, B., \& Majumdar, D. Das. (2014). Distinguishing Different Sedimentary Facies in a Deltaic System. Sedimentary Geology, 308, 53-62.

https://doi.org/10.1016/j.sedgeo.2014.05.001

Purtill, M. P. (2009). The Ohio Archaic: A Review. In T. E. Emerson, D. T. McElrath, \& A. C. Fortier (Eds.), Archaic Societies: Diversity and Diversity across the Midcontinent (pp. 565606). Albany: SUNY Press.

Purtill, M. P. (2012). A Persistent Place: A Landscape Approach to the Prehistoric Archaeology of the Greenlee Tract in Southern Ohio. Raleigh: Lulu Press, Inc.

Purtill, M. P. (2015). Ph.D. Proposal: Towards a better understanding of Paleoindian Native American settlement in southern Ohio: a multi-scalar approach. Morgantown.

Purtill, M. P. (2016). Aeolian and Fluvial Interaction in the Middle Ohio River Valley: New Geomorphic, Stratigraphic, and Sedimentological Evidence from Sandy Springs, Adams County, Ohio. In Geological Society of America Abstracts with Programs. Vol. 48, No.75, p.89. Denver.

Purtill, M. P. (2017). Reconsidering the Potential Role of Saline Springs in the Paleoindian Occupation of Sandy Springs, Adams County, Ohio. Journal of Archaeological Science: Reports, 13, 164-174. https://doi.org/10.1016/j.jasrep.2017.03.054

Purtill, M. P. (2017). Windblown Sediments and their Potential for Late Pleistocene/Holocene Site Burial: An Indiana and Ohio Example. Midcontinental Archaeological Conference, Annual Meeting. Indianapolis.

Purtill, M. P., \& Kite, J. S. (2015). Midwestern Sand Dunes, Geoarchaeology, and Lidar: Preliminary Geomorphic Landform Analysis of the Sandy Springs Paleoindian Site in the Upper Ohio River Valley. In Geological Society of America Abstracts with Programs. Vol. 47, No. 5, p.89 (Vol. 47). Madison: Geological.

Pye, K. (1982). Morphological Development of Coastal Dunes in a Humid Tropical Environment, Cape Bedford and Cape Flattery, North Queensland. Geografiska Annaler.Series A.Physical Geography, 64A, 213-227.

Pye, K. (1987). Aeolian Dust and Dust Deposits. London: Academic Press.

Pye, K., \& Tsoar, H. (2009). Aeolian Sand and Sand Dunes. Berlin: Springer.

Rawling, J. E. (2000). A Review of Lamellae. Geomorphology, 35, 1-9.

Rawling, J. E., Hanson, P. R., Young, A. R., \& Attig, J. W. (2008). Late Pleistocene Dune Construction in the Central Sand Plain of Wisconsin, USA. Geomorphology, 100(3-4), 494-505. https://doi.org/http://dx.doi.org/10.1016/j.geomorph.2008.01.017

Ray, C. E., Cooper, B. N., \& Benninghoff, W. S. (1967). Fossil Mammals and Pollen in a Late Pleistocene Deposit at Saltville, Virginia. Journal of Paleontology, 608-622.

Ray, L. L. (1974). Geomorphology and Quaternary Geology of the Glaciated Ohio River Valley: A Reconnaissance Study: a Study of the Geomorphic Development and Drainage Modifications Resulting from the Quaternary Glaciations of the Ohio River Valley, from Above Cincinnati, Oh. Washington: US Government Printing Office.

Redmond, B. G., \& Tankersley, K. B. (2005). Evidence of Early Paleoindian Bone Modification and Use at the Sheriden Cave Site (33WY252), Wyandot County, Ohio. American Antiquity, 70(3), 503-526.

Reimer, P. J., Bard, E., Bayliss, A., Beck, J. W., Blackwell, P. G., Ramsey, C. B., ... van der Plicht, J. (2013). IntCal13 and Marine13 Radiocarbon Age Calibration Curves 0-50,000 
Years cal BP. Radiocarbon, 55(4), 1869-1887. https://doi.org/10.2458/azu_js_rc.55.16947

Resources, O. D. of N. (2016). Rare Plants in Ohio. Retrieved from $\mathrm{http}: / /$ naturepreserves.ohiodnr.gov/rareplants

Ridges, M. (2006). Regional Dynamics of Hunting and Gathering: An Australian Case Study Using Archaeological Predictive Modeling. In M. W. Mehrer \& K. L. Westcott (Eds.), GIS and Archaeological Site Location Modeling (pp. 115-134). Boca Raton: Taylor \& Francis.

Riley, S. J., DeGloria, S. D., \& Elliot, R. (1999). A Terrain Ruggedness Index that Quantifies Topographic Heterogeneity. Intermountain Journal of Sciences, 5(1-4), 23-27.

Ritchie, W. A. (1965). The archaeology of New York State. Garden City: Natural History Press. Retrieved from https://www.amazon.com/archaeology-New-York-

State/dp/B0007DLCXE/ref=sr_1_1?s=books\&ie=UTF8\&qid=1521410413\&sr=1$1 \&$ keywords $=$ The + archaeology + of + New + York + STate

Rittenour, T. M., Blum, M. D., \& Goble, R. J. (2007). Fluvial Evolution of the Lower Mississippi River Valley during the Last 100 k.y. Glacial Cycle: Response to Glaciation and Sea-level Change. Bulletin of the Geological Society of America, 119(5-6), 586-608. https://doi.org/10.1130/B25934.1

Rittenour, T. M., Goble, R. J., \& Blum, M. D. (2005). Development of an OSL Chronology for Late Pleistocene Channel Belts in the lower Mississippi Valley, USA. Quaternary Science Reviews, 24(23), 2539-2554.

Robinson, B. S., \& Ort, J. C. (2013). Spatial Organization at Bull Brook. In J. A. M. Gingerich (Ed.), In the Eastern Fluted Point Tradition (pp. 104-120). Salt Lake City: The University of Utah Press.

Robinson, B. S., Ort, J. C., Eldridge, W. A., Burke, A. L., \& Pelletier, B. G. (2009). Paleoindian Aggregation and Social Context at Bull Brook. American Antiquity, 74(3), 423-447.

Robinson, F. W., Crock, J. G., \& Dorshow, W. (2018). Paleoindian Sites, Site Patterning, and Travel Corridors along the Southern Arm of the Champlain Sea. In J. A. M. Gingerich (Ed.), In the Eastern Fluted Point Tradition, Volume II (pp. 326-350). Salt Lake City: The University of Utah Press.

Robinson, R. W. (2009). Holocene Landform Evolution and Natural Site Formation Processes at the West Blennerhassett Archaeological Site (46WD83-A), Wood County, West Virginia. Department of Geology and Geography, West Virginia University, Morgantown.

Rogers, R. L. (1990). Late Quaternary Stratigraphy and Geologic History of the Upper Ohio River Valley, Near Gallipolis Locks and Dam. West Virginia University, Department of Geology and Geography, Morgantown.

Rogerson, P. A. (2010). Statistical Methods for Geography: A Student's Guide (3rd ed.). London: Sage Publications, Ltd.

Rolingson, M. A. (1964). Paleo-Indian Culture in Kentucky: A Study based on Projectile Points. Lexington: University of Kentucky Press.

Roper, D., \& Lepper, B. T. (1991). Archaeological Data Recovery from Four Sites at the William H. Zimmer Generating Station, Clermont County, Ohio. Jackson: Commonwealth Cultural Resources Group, Inc.

Roskin, J., Tsoar, H., Porat, N., \& Blumberg, D. G. (2011). Palaeoclimate Interpretations of Late Pleistocene Vegetated Linear Dune Mobilization Episodes: Evidence from the Northwestern Negev Dunefield, Israel. Quaternary Science Reviews, 30(23-24), 33643380. https://doi.org/http://dx.doi.org/10.1016/j.quascirev.2011.08.014

Rostlund, E. (1960). The Geographic Range of the Historic Bison in the Southeast. Annals of the 
Association of American Geographers, 50(4), 395-407.

Rutledge, E. M., Holowychuk, N., Hall, G. F., \& Wilding, L. P. (1975). Loess in Ohio in

Relation to Several Possible Source Areas: I. Physical and Chemical Properties. Soil Science Society of America Journal, 39, 1125-1132.

https://doi.org/doi:10.2136/sssaj1975.03615995003900060031x

Ryan, P. A. (2010). Environmental effects of sediment on New Zealand streams: A review, New Zealand. Journal of Marine and Freshwater Research, 25(2), 207-221. https://doi.org/10.1080/00288330.1991.9516472

Sanborn, P., \& Pawluk, S. (1989). Microstructural Diversity in Ah Horizons of Black Chernozemic Soils, Alberta and British Columbia. Geoderma, 221-240.

Schaetzl, R. J. (1998). Lithologic Discontinuities in Some Soils on Drumlins: Theory, Detection, and Application. Soil Science, 163(7), 570-590.

Schaetzl, R. J., \& Anderson, S. (2005). Soils: Genesis and Geomorphology. Cambridge: Cambridge University Press.

Schmidlin, T. W. (1996). Climate and Weather. In L. Peacefull (Ed.), The Geography of Ohio (pp. 16-30). Kent: Kent State University Press.

Schoeneberger, P. J., Wysocki, D. A., Benham, E. C., \& Staff, S. S. (2012). Field Book for Describing and Sampling Soils, Version 3.0. Washington: U.S. Government Printing Office.

Schwan, J. (1986). The origin of horizontal alternating bedding in weichselian aeolian sands in Northwestern Europe. Sedimentary Geology, 49(1-2), 73-108. https://doi.org/10.1016/0037-0738(86)90016-3

Seeman, M. F. (1994). Intercluster Lithic Patterning at Nobles Pond: A Case for" Disembedded" Procurement among Early Paleoindian Societies. American Antiquity, 59(2), 273-288.

Seeman, M. F., Loebel, T. J., Comstock, A., \& Summers, G. L. (2013). Working with Wilmsen: Paleoindian End Scraper Design and Use at Nobles Pond. American Antiquity, 78(3), 407432.

Seeman, M. F., \& Prufer, O. H. (1982). An Updated Distribution of Ohio Fluted Points. Midcontinental Journal of Archaeology, 7(2), 155-169.

Seeman, M. F., \& Prufer, O. H. (1984). The Effects of Cultivation and Collecting on Ohio Fluted Point Finds: A Cautionary Note. Midcontinental Journal of Archaeology, 9(2), 227-233.

Seeman, M. F., Summers, G., Dowd, E., \& Morris, L. (1994). Fluted Point Characteristics at Three Large Sites: The Implications for Modeling Early Paleoindian Settlement Patterns in Ohio. In W. S. Dancey (Ed.), The First Discovery of America: Archaeological Evidence of the Early Inhabitants of the Ohio Area (pp. 77-94). Columbus: Ohio Archaeological Council.

Shane, L. C. K. (1994). Intensity and Rate of Vegetation and Climatic Change in the Ohio Region between 14,000 and 9,000 14C YBP. In W. S. Dancey (Ed.), The First Discovery of America: Archaeological Evidence of the Early Inhabitants of the Ohio Area (pp. 7-22). Columbus: The Ohio Archaeological Council.

Shane, L. C. K., Snyder, G. G., \& Anderson, K. H. (2001). Holocene Vegetation and Climate Changes in the Ohio Region. In O. H. Prufer, S. E. Pedde, \& R. S. Meindl (Eds.), Archaic Transitions in Ohio \& Kentucky Prehistory (pp. 11-58). Kent: Kent State University Press.

Shane Miller, D. (2016). Modeling Clovis Landscape Use and Recovery Bias in the Southeastern United States Using the Paleoindian Database of the Americas (PIDBA). American Antiquity, 81(4), 697-716.

Shaw, P. A., \& Bryant, R. G. (2011). Pans, Playas and Salt Lakes. In D. S. G. Thomas (Ed.), 
Arid Zone Geomorphology: Process, Form and Change in Drylands (3rd ed., pp. 373-401). Oxford: Wiley-Blackwell.

Sholts, S. B., Stanford, D. J., Flores, L. M., \& Wärmländer, S. K. T. S. (2012). Flake Scar Patterns of Clovis Points Analyzed with a New Digital Morphometrics Approach: Evidence for Direct Transmission of Technological Knowledge across early North America. Journal of Archaeological Science, 39(9), 3018-3026. https://doi.org/10.1016/j.jas.2012.04.049

Shott, M. J. (2002). Sample Bias in the Distribution and Abundance of Midwestern Fluted Bifaces. Midcontinental Journal of Archaeology.

Shott, M. J. (2004). Hunter-gatherer Aggregations in Theory and Evidence: The Eastern North American Paleoindian Case. In G. M. Crothers (Ed.), Hunters and Gatherers in Theory and Archaeology (Vol. Occasional, pp. 68-102). Carbondale: Southern Illinois University.

Shuman, B., Webb III, T., Bartlein, P., \& Williams, J. W. (2002). The Anatomy of a Climatic Oscillation: Vegetation Change in Eastern North America during the Younger Dryas Chronozone. Quaternary Science Reviews, 21(16), 1777-1791.

Sillitoe, P., \& Hardy, K. (2003). Living Lithics: Ethnoarchaeology in Highland Papua New Guinea. Antiquity, 77(297), 555-566.

Simard, C. M. (1989). Geologic History of the Lower Terraces and Floodplains of the Upper Ohio River Valley. Department of Geology and Geography, West Virginia University, Morgantown.

Simpson, L. C., \& Florea, L. J. (2009). The Cumberland Plateau of Eastern Kentucky. In A. N. Palmer \& M. V Palmer (Eds.), Caves and Karst of America (Volume 1, pp. 70-79). Huntsville: National Speleological Society.

Singhvi, A. K., Bluszcz, A., Bateman, M. D., \& Rao, M. S. (2001). Luminescence dating of loess-palaeosol sequences and coversands: Methodological aspects and palaeoclimatic implications. Earth-Science Reviews, 54(1-3), 193-211. https://doi.org/10.1016/S00128252(01)00048-4

Sitzia, L., Bertran, P., Sima, A., Chery, P., Queffelec, A., \& Rousseau, D.-D. (2017). Dynamics and Sources of Last Glacial Aeolian Deposition in Southwest France derived from Dune Patterns, Grain-size Gradients and Geochemistry, and Reconstruction of Efficient Wind Directions. Quaternary Science Reviews, 170, 250-268. https://doi.org/https://doi.org/10.1016/j.quascirev.2017.06.029

Slucher, E. R., Swinford, E. M., Larsen, G. E., Schumacher, G. A., Shrake, D. K., Rice, C. K., ... Rea, R. G. (2006). Bedrock Geologic Map of Ohio, BG-1, Version 6. Columbus: Ohio Department of Natural Resources, Division of Geological Survey.

Smallwood, A. (2012). Clovis Technology and Settlement in the American Southeast: Using Biface Analysis to Evaluate Dispersal Models. American Antiquity, 77(4), 689-713.

Smith, E. E. (1990). Paleo-Indian Settlement and Lithic Procurement Patterns in the Karstic Region of Southcentral Indiana. Indiana University, Department of Anthropology, Bloomington.

Smith, E. E. (1990). Paleoindian Economy and Settlement Patterns in the Wyandotte Chert Source Area, Unglaciated South-Central Indiana. Early Paleoindian Economies of Eastern North America, 5, 217-258.

Smith Jr, E. E. (1995). The Magnet Site (12PE171): A Late Paleoindian Site in Perry County, Southern Indiana. Southeastern Archaeology, 14(2), 158-181.

Soil Survey Staff. (1999). Soil Taxonomy. A Basic System of Soil Classification for Making and Interpreting Soil Surveys. Washington: U.S. Department of Agriculture, Natural Resources 
Conservation Service.

Speth, J. D., Newlander, K., White, A. A., Lemke, A. K., \& Anderson, L. E. (2013). Early Paleoindian Big-game Hunting in North America: Provisioning or Politics? Quaternary International, 285, 111-139.

Spiess, A., Cowie, E., \& Bartone, R. (2012). Geographic Clusters of Fluted Point. In C. Chapdelaine (Ed.), Late Pleistocene Archaeology \& Ecology in the Far Northeast (pp. 95112). College Station: Texas A \& M University Press.

Stafford, C. R. (2004). Modeling soil-geomorphic Associations and Archaic Stratigraphic Sequences in the Lower Ohio River Valley. Journal of Archaeological Science, 31(8), 1053-1067. https://doi.org/http://dx.doi.org/10.1016/j.jas.2004.01.004

Stafford, C. R., \& Creasman, S. D. (2002). The Hidden Record: Late Holocene Landscapes and Settlement Archaeology in the Lower Ohio River Valley. Geoarchaeology, 17(2), 117-140.

Staubwasser, M., Sirocko, F., Grootes, P. M., \& Segl, M. (2003). Climate Change at the 4.2 ka BP Termination of the Indus Valley Civilization and Holocene South Asian Monsoon Variability. Geophysical Research Letters, 30, 1425-1428. https://doi.org/10.1029/2002GL016822

Stephen, I. (1960). Clay Orientation in Soils. Science Progress, 48, 322-331.

Stevens, J. P. (2002). Applied Multivariate Statistics for the Social Sciences (4th ed.). Mahwah: Lawrence Erlbaum Associates.

Stone, A. E. C., \& Thomas, D. S. G. (2008). Linear Dune Accumulation Chronologies from the Southwest Kalahari, Namibia: Challenges of Reconstructing Late Quaternary Palaeoenvironments from Aeolian Landforms. Quaternary Science Reviews, 27(17-18), 1667-1681. https://doi.org/http://dx.doi.org/10.1016/j.quascirev.2008.06.008

Stone, K. H. (1972). A geographer's strength: the multiple-scale approach. Journal of Geography, 71(6), 354-362.

Stoops, G. (2003). Guidelines for Analysis and Description of Soil and Regolith Thin Sections. Madison: Soil Science Society of America.

Stoops, G. (2010). Interpretation of Micromorphological Features of Soils and Regoliths. Oxford: Elsevier.

Stothers, D. M. (1996). Resource procurement and band territories: A model for lower Great Lakes Paleoindian and Early Archaic settlement systems. Archaeology of Eastern North America, 24, 173-216. Retrieved from http://www.jstor.org/stable/40914410

Stout, J. C., \& Belmont, P. (2013). TerEx Toolbox for Semi-automated Selection of Fluvial Terrace and Floodplain Features from LiDAR. Earth Surface Processes and Landforms, 39(5), 569-580. https://doi.org/10.1002/esp.3464

Stout, W., \& Schoenlaub, R. A. (1945). The Occurrence of Flint in Ohio. Bulletin, Geological Survey of Ohio, Fourth Series. Columbus: Geological Survey of Ohio.

Stout, W., Ver Steeg, K., \& Lamb, G. F. (1943). Geology of Water in Ohio:(a Basic Report). Columbus: Geological Survey of Ohio.

Stout, Lamborn, Raymond E., Schaaf, Downs., W. (1932). Brines of Ohio (Vol. Bulletin 3). Columbus: Geological Survey of Ohio.

Surovell, T. A., \& Waguespack, N. M. (2009). Human Prey Choice in the Late Pleistocene and its Relation to Megafaunal Extinctions. In G. Haynes (Ed.), American Megafaunal Extinctions at the End of the Pleistocene (pp. 77-105). New York: Springer.

Survey, K. G. (2016). Kentucky Geological Survey Geologic Map Service. Retrieved from http://kgs.uky.edu/kgsmap/kgsgeoserver/viewer.asp 
Tankersley, K. B. (1985). The Potential for Early Man Sites at Big Bone Lick, Kentucky. Tennessee Anthropologist, 10, 27-49.

Tankersley, K. B. (1990). Late Pleistocene Lithic Exploitation in the Midwest and Midsouth: Indiana, Ohio, and Kentucky. Research in Economic Anthropology Supplement, 5, 259-302.

Tankersley, K. B. (1996). Ice Age Hunters and Gatherers. In R. B. Lewis (Ed.), Kentucky Archaeology (pp. 21-38). Lexington: The University of Kentucky Press.

Tankersley, K. B. (1998). Variation in the Early Paleoindian Economies of Late Pleistocene Eastern North America. American Antiquity, 63(1), 7-20.

Tankersley, K. B. (2007). Stop 1: Big Bone Lick, Kentucky - Late Pleistocene Archaeology. In T. S. Dalbey (Ed.), Guidebook No. 11: Geological Aspects of Key Archaeological Sites in Northern Kentucky and Southern Ohio (pp. 45-49). Columbus: Geological Society of America.

Tankersley, K. B., \& Isaac, B. L. (1990). Early Paleoindian Economies of Eastern North America. Greenwich: Jai Press.

Tankersley, K. B., Murari, M. K., Crowley, B. E., Owen, L. A., Storrs, G. W., \& Mortensen, L. (2015). Quaternary chronostratigraphy and stable isotope paleoecology of Big Bone Lick, Kentucky, USA. Quaternary Research, 83(3), 479-487. https://doi.org/http://dx.doi.org.www.libproxy.wvu.edu/10.1016/j.yqres.2015.01.009

Tankersley, K. B., Smith, E. E., \& Cochran, D. R. (1990). Early Paleoindian Land Use, Mobility, and Lithic Exploitation Patterns: An Updated Distribution of Fluted Points in Indiana. North American Archaeologist, 11(4), 301-319.

Tankersley, K. B., Waters, M. R., \& Stafford Jr, T. W. (2009). Clovis and the American Mastodon at Big Bone Lick, Kentucky. American Antiquity, 74(3), 558-567.

Tankersley, K.B., Munson, P. J., \& Tankersley, J. R. (1983). The Archaeological Geology of the Whitewater-Great Miami-Ohio River Confluence Area. In 96th Annual Meeting of the Geological Society of America. Indianapolis.

Telfer, M. W., \& Thomas, D. S. G. (2007). Late Quaternary Linear Dune Accumulation and Chronostratigraphy of the Southwestern Kalahari: Implications for Aeolian Palaeoclimatic Reconstructions and Predictions of Future Dynamics. Quaternary Science Reviews, 26(1921), 2617-2630. https://doi.org/10.1016/j.quascirev.2007.07.006

Thomas, D. S. G. (2011). Aeolian Landscapes and Bedforms. In D. S. G. Thomas (Ed.), Arid Zone Geomorphology: Process, Form and Change in Drylands (3rd ed., pp. 427-454). Oxford: Wiley-Blackwell.

Thomas, D. S. G. (2013). Reconstructing Paleoenvironments and Palaeoclimates in Drylands: What can Landform Analysis Contribute? Earth Surface Processes and Landforms, 38, 3 16.

Thornthwaite, Charles, W. (1931). The Climate of North America according to a New Classification. Geographical Review, 21, 633-655.

Thorson, R. M., \& Schile, C. A. (1995). Deglacial Eolian Regimes in New England. Washington, D.C.: Geological Society of America Bulletin 107.

Tobin, B. D., \& Weary, D. J. (2004). Digital engineering aspects of Karst map: a GIS version of Davies, W.E., Simpson, J.H., Ohlmacher, G.C., Kirk, W.S., and Newton, E.G., 1984, Engineering aspects of Karst: U.S. Geological Survey, National Atlas of the United States of America, Scale 1:7,50.

Todd, D. K., \& Mays, L. W. (2005). Groundwater Hydrology. Hoboken: John Wiley \& Sons, Inc. 
Tornqvist, T. E., Bick, S. J., Gonzalez, J. L., van der Borg, K., \& de Jong, A. F. M. (2004). Tracking the Sea-level Signature of the 8.2 ka Cooling Event: New Constraints from the Mississippi Delta. Geophysical Research Letters, 31, L23309. Retrieved from doi:10.1029/\%0A2004GL021429

Tune, J. W. (2016). Characterizing Cumberland Fluted Biface Morphology and Technological Organization. Journal of Archaeological Science, Reports, 6, 310-320.

Twenhofel, W. H., \& Tyler, S. A. (1941). Methods of Study of Sediments. New York: McGrawHill.

U.S. Department of Agricuture, N. R. C. S. (2017). Geospatial Data Gateway. Retrieved June 6, 2017, from https://gdg.sc.egov.usda.gov/

U.S. Fish \& Wildlife. (2017). The National Wetlands Inventory.

U.S. Geological Survey. (2013). The National Map. Retrieved June 6, 2017, from http://nationalmap.gov/viewer.html

U.S. Geological Survey. (2017). Hydrography. Retrieved June 6, 2017, from https://nhd.usgs.gov/index.html

Natural Resources Conservation Service. (2017). Web Soil Survey. Retrieved June 3, 2017, from http://websoilsurvey.nrcs.usda.gov/.

Vepraskas, M. J., \& Wilson, M. A. (2008). Soil Micromorphology: Concepts, Techniques, and Applications. In A. L. Ulery \& L. R. Drees (Eds.), Methods of Soil Analysis: Part 5 Mineralogical Methods (pp. 191-226). Madison: American Society of Agronomy, SSSA Book Series 5.

Vincent, M. A., Gardner, R. L., \& Riley, B. P. (2011). Additions to and Interesting Records for the Ohio Vascular Flora (with One New Record for Indiana). Phytoneuron, 60, 1-23.

Vincent, P. (1986). Differentiation of modern beach and coastal dune sands - a logistic regression approach using the parameters of the hyperbolic function. Sedimentary Geology, $49,167-176$.

Waguespack, N. M., \& Surovell, T. A. (2003). Clovis Hunting Strategies, or How to Make out on Plentiful Resources. American Antiquity, 68(2), 333-352. Retrieved from http://www.jstor.org/stable/3557083

Walton, N. R. G. (1989). Electrical conductivity and total dissolved solids - what is their precise relationship? Desalination, 72(3), 275-292.

Wang, H., Curry, B., Stumpf, A., Miao, X., Waninger, S., Hanson, P., \& Goble, R. (2011). Evidence of Regional Drought from Dune and Loess Records during the Early Stage of LIS Retreat in the Midwestern United States. In AGU Fall Meeting. San Francisco, California, December 5-9.: American Geophysical Union.

Wang, H., Stumpf, A. J., Miao, X., \& Lowell, T. V. (2012). Atmospheric Changes in North America during the Last Deglaciation from Dune-Wetland Records in the Midwestern United States. Quaternary Science Reviews, 58, 124-134.

Warren, R. E., \& Asch, D. L. (2003). A Predictive Model of Archaeological Site Location in the Eastern Prairie Peninsula. In K. L. Westcott \& R. J. Brandon (Eds.), Practical Applications of GIS for Archaeologists: a Predictive Modelling Toolkit (pp. 5-32). London: Taylor \& Francis.

Water, M.R. (2004). The Role of Geoarchaeology in the Search for the First Americans. In B.T. Lepper \& R. Bonnichsen (Eds.), New Perspectives on the First Americans (pp. 187-192). College Station: Texas A \& M University Press.

Waters, M. R., \& Stafford, T. W. (2007). Redefining the Age of Clovis: Implications for the 
Peopling of the Americas. Science, 315(5815), 1122-1126.

https://doi.org/10.1126/science.1137166

Weeks, H. P., \& Kirkpatrick, C. M. (1976). Adaptations of white-tailed Deer to Naturally Occurring Sodium Deficiencies. The Journal of Wildlife Management, 40(4), 610-625.

Weiss, A. (2001). Topographic Position and Landforms Analysis. In Poster presentation, ESRI User Conference (p. 200). San Diego.

Wells, G. L. (1983). Late-glacial Circulation Over Central North America revealed by Aeolian Features. In F. A. Street-Perrott, M. Beran, \& R. Ratcliffe (Eds.), Variations in the Global Water Budget (pp. 317-330). Oxford: Springer.

Werner, A., Jones, K., Miller, G. L., Buchanan, B., Boulanger, M. T. ., Key, A. J. M. ., ... Eren, M. I. (2017). The morphometrics and microwear of a small Clovis assemblage from Guernsey County, Southeastern Ohio, U.S.A. Journal of Archaeological Science: Reports, $15,318-329$.

Wilcox, F. N. (1970). Ohio Indian Trails: A Pictorial Survey of the Indian Trails of Ohio. Kent: Kent State University Press.

Willard, D. A., Bernhardt, C. E., Korejwo, D. A., \& Meyers, S. R. (2005). Impact of millennialscale Holocene Climate Variability on Eastern North American Terrestrial Ecosystems: Pollen-based Climatic Reconstruction. Global and Planetary Change, 47(1), 17-35. https://doi.org/10.1016/j.gloplacha.2004.11.017

Witthoft, J. (1954). A Note on Fluted Point Relationships. American Antiquity, 19(3), 271-273. https://doi.org/10.2307/277132

Wong, D. W. S., \& Lee, J. (2005). Statistical Analysis of Geographic Information with ArcView GIS and ArcGIS. Hoboken: Wiley.

Wright, D. K., Forman, S. L., Waters, M. R., \& Ravesloot, J. C. (2011). Holocene Eolian Activation as a Proxy for Broad-scale Landscape Change on the Gila River Indian Community, Arizona. Quaternary Research, 76(1), 10-21. https://doi.org/http://dx.doi.org/10.1016/j.yqres.2011.04.008

Yu, Z. (2000). Ecosystem Response to Late Glacial and Early Holocene Climate Oscillations in the Great Lakes Region of North America. Quaternary Science Reviews, 19(17), 17231747.

Yu, Z., \& Eicher, U. (2001). Three Amphi-Atlantic Century-scale Cold Events during the Bølling-Allerød Warm Period. Géographie Physique et Quaternaire, 55(2), 171-179.

Zhou, N., Zhang, C.-L., Wu, X.-X., Wang, X., \& Kang, L. (2014). The Geomorphology and Evolution of Aeolian Landforms within a River Valley in a Semi-humid Environment: A Case Study from Mainling Valley, Qinghai-Tibet Plateau. Geomorphology, 224, 27-38. 
APPENDIX A: PUBLISHED JOURNAL ARTICLES 